\title{
An Active Inductor and Tunable Bandpass Filter in GaN
}

by

\author{
Tom Herbert
}

A thesis submitted to the Faculty of Graduate and Post Doctoral Affairs in partial fulfillment of the requirements for the degree of

\author{
Master of Applied Science
}

in

Electrical and Computer Engineering

\section{Carleton University, Ottawa, Canada}

\author{
(C) 2018 \\ Tom Herbert
}




\section{Abstract}

This thesis presents the design and implementation of an active inductor using gallium nitride technology. This design is largely motivated by the lack of high-quality varactors in GaN. Active inductors are therefore proposed as an alternative to varactors as an impedance tuning mechanism in $\mathrm{GaN}$ which will act as the building block for tunable circuits such as VCOs, filters, phase shifters etc. The fabrication of such tunable circuits in GaN will in turn allow for highly integrated complete RF systems on chip in GaN.

Furthermore, the design and implementation of a tunable bandpass filter with amplitude and frequency control making use of the aforementioned active inductor are presented. The proposed active inductor and filter were fabricated in a $0.5 \mu \mathrm{m}$ pHEMT GaN process. The active inductor's measured tuning range at $3.5 \mathrm{GHz}$ is 8 to $20 \mathrm{nH}$ with a quality factor greater than 200 . The measured tuning range of the active filter is $500 \mathrm{MHz}$ with an $\mathrm{S} 21$ of $3 \mathrm{~dB}$. The active inductor occupies an active area of $350 \mathrm{um}$ by $175 \mathrm{um}$. Fixed passive inductors of similar inductance occupy areas of approximately $350 \mathrm{um}$ by $350 \mathrm{um}$ (200\% increase compared to active inductor

area), while exhibiting extremely poor quality factors of less than 5. To the authors' knowledge, this is the first active inductor and bandpass filter implemented in GaN. 


\section{Acknowledgements}

Firstly, I would like to give a big thanks to my supervisors Professors Rony Amaya and Jim Wight. Not only would I like to thank them for their valuable advice and guidance but also for their patience and understanding in allowing me to work at a pace reflective of a part-time student with a young family and busy job.

I would also like to give thanks to Nagui Mikhail for his assistance with providing testing equipment and general lab and computer support. Also in the department, I would like to thank Anna Lee and Blazenka Power for their kind help and support.

A big shout out to the team over at CMC particularly James Dietrich who was a great help in getting the design to fabrication free of design violations.

On a more personal side, I would like to thank my wife for all her support and sacrifices while I undertook this effort. I'd also like to thank my wife for holding up the fort all the days and keeping things going while I was plugging away at this.

I'd like to thank my son, who puts a smile on my face every day and without whom I probably would have been finished a couple years ago!

To my mother, I would like to thank her for all the love and support she has given me over the years and who's hard work ethic inspired me to get over the finish line.

Finally, I would like to dedicate this work to my late father. Growing up in harsh conditions, he was forced to drop out of school at an early age yet somehow later still managed to put himself through graduate school in physics and then later formed a company that provided many jobs for many years. His determination and pigheadedness in the face of adversity inspire me every day. 


\section{Contents}

$\begin{array}{lll}\text { Abstract } & \text { i }\end{array}$

Acknowledgements ii

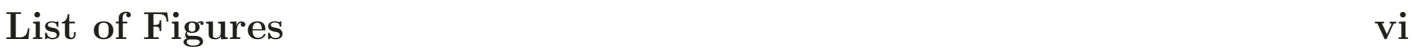

List of Tables viii

Abbreviations $\quad$ ix

1 Introduction $\quad 1$

1.1 Motivation and Overview . . . . . . . . . . . . . . 1

1.2 Thesis Contributions . . . . . . . . . . . . . . . . . 4

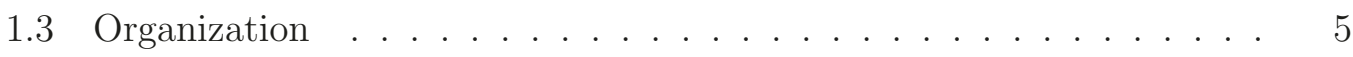

2 Background $\quad 7$

2.1 GaN Semiconductor Material . . . . . . . . . . . . . . . . 7

2.2 Tunable Devices In GaN / MMIC . . . . . . . . . . . . . . . . . . . . . . . 11

2.2.1 Switched Reactance. . . . . . . . . . . . . . . . . . . . . 12

2.2 .2 Varactors ...................... . . . . 13

2.3 Active Inductor Background . . . . . . . . . . . . . . . . . . . 15

2.3.1 Active Inductor / Gyrator Theory of Operation . . . . . . . . 17

2.3.2 Previous Works . . . . . . . . . . . . . . . . . . 21

2.4 Summary ............................... 24

3 Design $\quad 26$

3.1 Gan500 Design Kit . . . . . . . . . . . . . . . . . . . . . . . 26

3.2 Design Goals . . . . . . . . . . . . . . . . . . . . . . . . . . . 29

3.2.1 $\quad \mathrm{F}_{\mathrm{t}}$ and Selection of Operating Frequency . . . . . . . . . . . 29

3.3 General Design Considerations . . . . . . . . . . . . . . . . . . . 31

3.3.1 High Frequency Operation and Design Kit Limitations . . . . 31

3.4 Active Inductor Topology Selection . . . . . . . . . . . . . . . . . . . . . . 32

3.4.1 Common Source - Common Gate Topology . . . . . . . . . . . 33

3.4.2 Common Source - Common Drain Topology . . . . . . . . . . 36

3.5 Active Inductor Design . . . . . . . . . . . . . . . . . . . . 38

3.6 Bandpass Filter Design . . . . . . . . . . . . . . . . . . . . . . . 46

3.7 Summary . . . . . . . . . . . . . . . . . . . 50 
4 Results $\quad 55$

4.1 General Equipment Setup . . . . . . . . . . . . . . . . 56

4.2 Device Characterization . . . . . . . . . . . . . . . 58

4.2.1 $V_{D S}$ versus $I_{D S}$ Characterization . . . . . . . . . . . . 60

$4.2 .2 f_{t}$ Characterization . . . . . . . . . . . . . 62

4.3 Active Inductor Characterization Setup and Processing . . . . . . . . 69

4.3.1 Setup and Post Processing . . . . . . . . . . . . . . . . 69

4.3 .2 Active Inductor Results . . . . . . . . . . . . . . . . . 71

4.3.2.1 Comparison of Active Inductor Simulated and Measured results . . . . . . . . . . . . . 75

4.3.3 Bandpass Filter Results . . . . . . . . . . . . . . . 76

4.3.3.1 Comparison of Bandpass Filter Simulated and Mea-

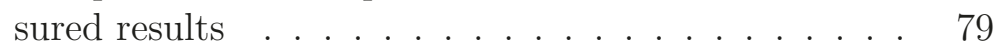

4.4 Summary . . . . . . . . . . . . . . . . . . . . . 81

5 Discussion $\quad \mathbf{8 3}$

5.1 Frequency Discrepancy Discussion . . . . . . . . . . . . . . . . 83

5.2 Contributions . . . . . . . . . . . . . . . . . 84

5.3 Future Work . . . . . . . . . . . . . . . . 86

$\begin{array}{lr}\text { Bibliography } & 88\end{array}$

$\begin{array}{ll}\text { A } & 94\end{array}$

A.1 Device $1 \ldots \ldots \ldots \ldots \ldots$. . . . . . . . . . . . . . . . . . . . 94

A.2 Device $2 \ldots \ldots \ldots \ldots . \ldots \ldots$

$\begin{array}{ll}\text { B } & 104\end{array}$

B.1 sweepDAC.m . . . . . . . . . . . . . . . . . . . . . . 104

B.2 GAN_IDvsVDS.m . . . . . . . . . . . . . . . . . 109

B.3 GAN_CZ.m . . . . . . . . . . . . . . . . . 111

B.4 getPNASparam.m . . . . . . . . . . . . . . . . 115

B.5 initPNA.m. . . . . . . . . . . . . . . . 117

B.6 initDAC.m . . . . . . . . . . . . . . . . . . 120

B.7 init_34401A.m . . . . . . . . . . . . . . . . . 121

B.8 read_34401A.m . . . . . . . . . . . . . . . . . . . . 122

B.9 dataViewer_freq2.m . . . . . . . . . . . . . . . . . . . 123

B.10 dataViewer_VTUNE1.m . . . . . . . . . . . . . . . . . . . 131

B.11 dataViewer_VTUNE2.m . . . . . . . . . . . . . . . . . . 138 
B.12 BPF_Viewer_freq2.m . . . . . . . . . . . . . . . . 145 


\section{List of Figures}

1.1 Typical Transceiver RF Front End . . . . . . . . . . . . . . . 3

2.1 Performance of Semiconductor materials across frequency and power[6] 11

2.2 Typical example of digitally tuned capacitor[18] . . . . . . . . . . . . 13

2.3 Typical GaN/AlGaN planar topology $[20] \ldots \ldots$. . . . . . . . . . . 14

2.4 Operation of the MSM-2DEG varactor a) unbiased device; b) $\mathrm{V}_{\text {BIAS }}$

$=-\mathrm{V}_{\text {-th }}$; c) $\mathrm{V}_{\text {BIAS }}<<-\mathrm{V}_{\text {-th }}$; d) voltage / capacitance relationship [19] . 14

2.5 Block diagram of ideal gyrator . . . . . . . . . . . . . . . . . . . 17

2.6 Block Diagram of Non Ideal Gyrator . . . . . . . . . . . . . . . . . . . . . 20

2.7 Equivalent Circuit Of Non Ideal Active Inductor . . . . . . . . . . . . 21

3.1 NRC GaN500 HFET Structure . . . . . . . . . . . . . . . . . . 27

3.2 Basic common source-common gate topology . . . . . . . . . . . . . 33

3.3 Common source-common gate layout . . . . . . . . . . . . . . 35

3.4 Common source-common gate Simulated Frequency Performance . . . 36

3.5 Basic common source-common drain topology . . . . . . . . . . . . 37

3.6 Basic common source-common drain simulated results . . . . . . . . . 39

3.7 Cascode Amplifier - common drain topology . . . . . . . . . . . . . . 40

3.8 Cascode Amplifier - common drain simulated results . . . . . . . . . . 41

3.9 Schematic with real biasing components . . . . . . . . . . . . . . 42

3.10 Schematic with real biasing simulation results . . . . . . . . . . . . 43

3.11 Final Schematic Topology, active biasing introduced . . . . . . . . . . 44

3.12 Final Schematic Simulation Results . . . . . . . . . . . . . . . . . 45

$3.132 \mathrm{~mm}$ X 2mm Circuit Layout . . . . . . . . . . . . . . . . . . 46

3.14 Active Inductor Layout . . . . . . . . . . . . . . . . . . . . . . 47

3.15 Final Layout Co-Simulation; Inductance and Q vs Frequency . . . . . 48

3.16 Final Layout Co-Simulation; Inductance and Q vs VTUNE1 . . . . . 49

3.17 Final Layout Co-Simulation; Inductance and Q vs VTUNE2 . . . . . 50

3.18 Bandpass Filter Topology . . . . . . . . . . . . . . . . . . 50

3.19 Bandpass Filter Layout . . . . . . . . . . . . . . . . . . . 51

3.20 Bandpass Filter Simulated Tuning Range . . . . . . . . . . . . . 52

3.21 Bandpass Filter Simulated Pass Band . . . . . . . . . . . . . . . 53

3.22 Bandpass Filter Simulated Linearity . . . . . . . . . . . . . . . . . 54

4.1 View of fabricated IC under microscope $(2 \mathrm{~mm} \times 2 \mathrm{~mm}) \ldots \ldots$

4.2 Block diagram of ideal gyrator . . . . . . . . . . . . . . 56

4.3 Photograph of Test Setup . . . . . . . . . . . . . . . . . 58

4.4 Photograph of IC on probing station . . . . . . . . . . . . . . 59

4.5 Typical VNA capture of active inductor . . . . . . . . . . . . . . . . 60

4.6 Characteristic curve, device $1 \ldots \ldots$. . . . . . . . . . . . 61 
4.7 Characteristic curve, device $2 \ldots \ldots \ldots$. . . . . . . . . . . . 62

4.8 Characteristic curve, GaN500 V3.01 manual . . . . . . . . . . . . . 63

$4.9 \mathrm{Ft}$ vs h21, device $1, \mathrm{VDS}=10 \mathrm{~V}, \mathrm{VGS}=-3 \mathrm{~V} \ldots \ldots \ldots 6$

$4.10 \mathrm{Ft}$ vs $\mathrm{h} 21$, device 1 , VDS $=10 \mathrm{~V}$, VGS $=-2 \mathrm{~V} \ldots \ldots \ldots 4$

$4.11 \mathrm{Ft}$ vs h21, device $1, \mathrm{VDS}=10 \mathrm{~V}, \mathrm{VGS}=-1 \mathrm{~V} \ldots \ldots \ldots 6$

$4.12 \mathrm{Ft}$ vs h21, device 1 , VDS $=10 \mathrm{~V}$, VGS $=0 \mathrm{~V} \ldots \ldots \ldots 5$

$4.13 \mathrm{Ft}$ vs IDS, device 1 , $\mathrm{VDS}=10 \mathrm{~V} \ldots \ldots \ldots 66$

4.14 Ft vs. JDS, device $1, \mathrm{VDS}=5 \mathrm{~V}, 10 \mathrm{~V}, 15 \mathrm{~V}, 20 \mathrm{~V} \ldots \ldots \ldots$

4.15 Ft vs. JDS, device 2 , VDS $=5 \mathrm{~V}, 10 \mathrm{~V}, 15 \mathrm{~V}, 20 \mathrm{~V} \ldots \ldots \ldots$

4.16 Ft vs. JDS, Simulated, VDS $=5 \mathrm{~V}, 10 \mathrm{~V}, 15 \mathrm{~V}, 20 \mathrm{~V} \ldots \ldots . \ldots$

$4.17 \mathrm{~L}$ and $\mathrm{Q}$ vs Frequency data viewer applet . . . . . . . . . . . . . . 71

4.18 L and Q vs VTUNE1 data viewer applet . . . . . . . . . . . . . . . . . . 72

$4.19 \mathrm{~L}$ and Q vs VTUNE2 data viewer applet . . . . . . . . . . . . . 73

4.20 Typical Measured Active Inductor Response Across frequency . . . . 74

4.21 Measured Active Inductor parameters Across frequency . . . . . . . . 75

4.22 Measured Active Inductor Response Across VTUNE1 at $3.5 \mathrm{GHz}$. . 76

4.23 Measured Active Inductor Response Across VTUNE2 at $3.5 \mathrm{GHz}$. . 77

4.24 Measured Active Inductor Response of Device 1 and Device 2 . . . . 78

4.25 Measured Bandpass Filter Response . . . . . . . . . . . . . . . . . 79

4.26 Measured Bandpass Filter Passband Width . . . . . . . . . . . . . . 80

5.1 Active Inductor schematic modeling star capacitance . . . . . . . . . 84

5.2 Simulation results modelling stray capacitance at bias inductor . . . . 85

A.1 Ft vs IDS, device $1, \mathrm{VDS}=5 \mathrm{~V} \ldots \ldots \ldots \ldots . \ldots \ldots$

A.2 $\quad$ Ft vs IDS, device $1, \mathrm{VDS}=9 \mathrm{~V} \ldots \ldots \ldots \ldots . \ldots \ldots$

A.3 Ft vs IDS, device $1, \mathrm{VDS}=9.5 \mathrm{~V} \ldots \ldots \ldots \ldots . \ldots \ldots$

A.4 Ft vs IDS, device 1 , $\mathrm{VDS}=10 \mathrm{~V} \ldots \ldots \ldots . \ldots . \ldots 96$

A.5 Ft vs IDS, device 1 , $\mathrm{VDS}=10.5 \mathrm{~V} \ldots \ldots \ldots . \ldots 96$

A.6 $\quad$ Ft vs IDS, device 1 , $\mathrm{VDS}=11 \mathrm{~V} \ldots \ldots \ldots . \ldots . \ldots 97$

A.7 Ft vs IDS, device 1 , $\mathrm{VDS}=15 \mathrm{~V} \ldots \ldots \ldots . \ldots \ldots 7$

A.8 Ft vs IDS, device $1, \mathrm{VDS}=20 \mathrm{~V} \ldots \ldots \ldots \ldots$

A.9 Ft vs IDS, device 2 , $\mathrm{VDS}=5 \mathrm{~V} \ldots \ldots \ldots \ldots$

A.10 Ft vs IDS, device 2 , $\mathrm{VDS}=9 \mathrm{~V} \ldots \ldots \ldots \ldots . \ldots \ldots$

A.11 Ft vs IDS, device 2 , $\mathrm{VDS}=9.5 \mathrm{~V} \ldots \ldots \ldots . \ldots . \ldots 100$

A.12 Ft vs IDS, device 2 , VDS $=10 \mathrm{~V} \ldots \ldots \ldots \ldots . \ldots \ldots 10 \ldots$

A.13 Ft vs IDS, device 2 , VDS $=10.5 \mathrm{~V} \ldots \ldots \ldots \ldots 10 \ldots$

A.14 Ft vs IDS, device 2 , VDS $=11 \mathrm{~V} \ldots \ldots \ldots \ldots$

A.15 Ft vs IDS, device 2, $\mathrm{VDS}=15 \mathrm{~V} \ldots \ldots \ldots \ldots . \ldots \ldots$

A.16 Ft vs IDS, device 2 , $\mathrm{VDS}=20 \mathrm{~V} \ldots \ldots \ldots \ldots$ 


\section{List of Tables}

2.1 Physical Properties of Semiconductors for High Power Devices . . . . 8

2.2 Table of Previous Active Inductor Publications . . . . . . . . . . . . . 22

3.1 GaN500 Interconnect Properties . . . . . . . . . . . . . . . . . . 28

3.2 Active Inductor Topology Comparison . . . . . . . . . . . . . . 38

4.1 List of test equipment . . . . . . . . . . . . . . . . . . 57

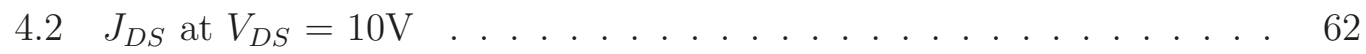

4.3 Summary of $\mathrm{ft}$ results for $\mathrm{VDS}=5 \mathrm{~V} \ldots \ldots \ldots \ldots$

4.4 Summary of $\mathrm{ft}$ results for $\mathrm{VDS}=10 \mathrm{~V} \ldots \ldots \ldots \ldots$. . . . . . . 67

4.5 Summary of $\mathrm{ft}$ results for $\mathrm{VDS}=15 \mathrm{~V} \ldots \ldots \ldots \ldots$

4.6 Summary of $\mathrm{ft}$ results for $\mathrm{VDS}=20 \mathrm{~V} \ldots \ldots \ldots$. . . . . . . 67

4.7 Active Inductor Simulated Vs. Measured Results . . . . . . . . . . . 76

4.8 Bandpass Filter Simulated Vs. Measured Results . . . . . . . . . . . 81 


\section{Abbreviations}

\begin{tabular}{|c|c|}
\hline AI & Active Inductor \\
\hline BW & Bandwidth \\
\hline CMOS & Complementary Metal Oxide Semiconductor \\
\hline DAC & Digital to Analog Converter \\
\hline VNA & Vector Network Analyzer \\
\hline $\mathrm{ft}$ & Transit Frequency \\
\hline FET & Field Effect Transistor \\
\hline GaAs & Gallium Arsenide \\
\hline GaN & Gallium Nitride \\
\hline HEMT & High Electron Mobility Transistor \\
\hline HFET & Heterostructure Filed Effect Transistor \\
\hline HPA & High Power Amplifier \\
\hline IC & Integrated Circuit \\
\hline LNA & Low Noise Amplifier \\
\hline MESFET & Metal Semiconductor Field Effect Transistor \\
\hline MIM & Metal-Insulator-Metal \\
\hline MMIC & Monolithic Microwave Integrated Circuit \\
\hline NRC & NRC National Research Council, Canada \\
\hline PA & Power Amplifier \\
\hline PDK & Process Design Kit \\
\hline Q & Quality Factor \\
\hline RFIC & Radio Frequency Integrated Circuit \\
\hline Si & Silicon \\
\hline
\end{tabular}




\section{Chapter 1}

\section{Introduction}

This thesis is a study and report on the implementation of active inductors in Gallium Nitride MMICs. In particular, this report focuses upon active inductors for use in tunable circuits in GaN MMICs. This thesis presents the complete design, simulation and implementation of an active inductor in GaN. Furthermore, this thesis presents the design and implementation of a tunable bandpass incorporating said active inductor as a means of tuning.

\subsection{Motivation and Overview}

Wireless electronic communication has been in use for over a century now. For most of that period wireless communication was used in conventional applications such as radio / television broadcast, HAM radio, satellite communications, radar / navigation among others. Ever since the adoption of the mobile telephone and the internet by the general public several decades ago both the number of wireless applications and number of wireless users has increased drastically to the point where wireless / mobile devices are ubiquitous in everyday life.

A typical mobile phone will communicate across several frequency bands just for cellular coverage. In addition to cellular coverage, a typical mobile phone will have transceivers in the $2.4 \mathrm{GHz}$ and $5 \mathrm{GHz}$ ISM bands for WIFI and Bluetooth communication. Homes and offices are now cluttered with signals in the various unlicensed 
ISM bands as everything from computers, tablets, TVs, headphones, and various other appliances and gadgets become wirelessly interconnected.

Radio is also widely used in automobiles and transportation in general. Cars already use GPS for navigation, and radar at $24 \mathrm{GHz}$ (moving to $80 \mathrm{GHz}$ ) for assisted driving features such as collision avoidance. As transportation becomes more autonomous vehicles will make use of radio technology increasingly for navigation and detection systems and also for communications in smart traffic systems.

The list of radio applications goes on. It is used in inventorying systems and location systems in warehousing and retail (e.g. RFID), medical and health care monitoring devices, security and so on.

This increase in wireless applications and wireless users is the driving force behind several design requirements

- The need to lower hardware costs. It is therefore desirable to produce highly integrated ICs that contain complete front-end RF / microwave functionality and if possible baseband digital functionality also

- The need to optimize radio spectrum to maximize channel capacity. This requires transceivers to be adaptable in among other factors frequency, physical environment, dynamic range etc. This high-level adaptive behaviour requires low-level adaptive circuit blocks.

- The need to reduce power consumption to prolong battery use

A two-way radio system is required to both send and receive information. In modern systems, the transmit and receive functionality is combined in a device termed a transceiver. Depending on one's perspective, the transceiver may pertain to the 
entire transmit / receiver chain from baseband (the actual information bearing signal) to the up-converted radio frequency signal and also frequencies in between termed intermediary frequencies or IF for short. For this discussion, a transceiver shall only consist of the circuit blocks operating at the transmitted or received radio frequency.

Transceivers come in many variations, but a typical transceiver RF front end is shown in Figure 1.1

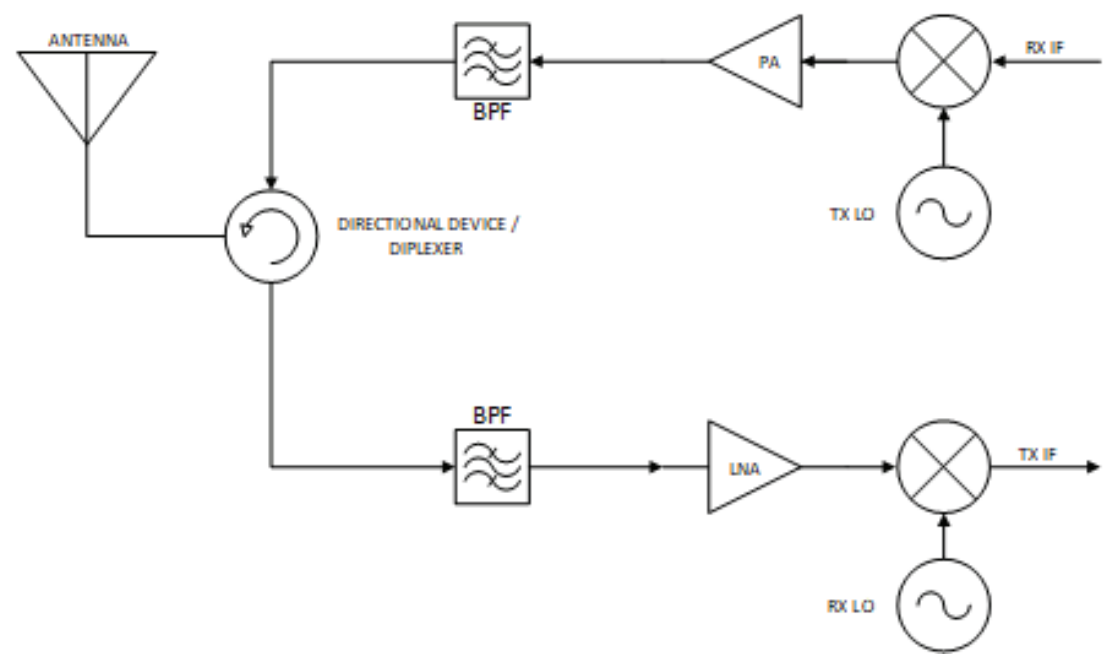

Figure 1.1: Typical Transceiver RF Front End

Complete transceiver on chip devices have typically been the domain of CMOS technology. Although it is not the optimum technology for RF / microwave circuits, it is a mature technology that is well understood and modeled and can easily be integrated along with digital circuitry to deliver complete system on chip devices. However, for all its benefits, due to its low breakdown voltage, CMOS faces many challenges in producing large output power efficiently. For this reason, alternate materials are explored. GaN is one such material. GaN has long been recognized for both its high power and high frequency capability, typically being used to implement devices requiring high linearity such as power amplifiers. 
Observing Figure 1.1, it is clear that all but the most simple transceivers would require some degree of tuning, whether in the local oscillator, PA matching, or bandpass filter center frequency for instance. Typically, tunable circuits are implemented using varactors. As discussed in detail further along in this thesis, varactors implemented in GaN exhibit high series resistance making them unsuitable for implementing tunable circuits which require a high quality factor e.g. VCOs, narrow band filters. Therefore, researching an alternative tuning mechanism in GaN to the varactor is the motivation behind this thesis. More specifically, the active inductor as a tuning mechanism in GaN is the primary motivation of this thesis.

Active inductors in GaN will also be subject to the same high contact resistance as varactors. However, active inductors typically employ some form of negative resistance which can be used to reduce the contact resistance. Along with the inherent low on resistance of GaN material, GaN potentially could be an excellent candidate for providing low resistance / high Q active inductors.

\subsection{Thesis Contributions}

As a result of the challenges presented in the previous section in designing tunable circuits in GaN, this thesis provides the following contributions

- The design and fabrication of a tunable active inductor in GaN with a nominal $5 \mathrm{GHz}$ operation. To the best of the author's knowledge, there is no published research concerning active inductors in $\mathrm{GaN}$ which highlights the novelty of this work. 
- To demonstrate the use of the active inductor in a practical application. Specifically, the design and fabrication of a tunable bandpass filter in GaN incorporating said active inductor. Once again, to the best of the author's knowledge, there is no published research regarding tunable bandpass filters in GaN.

- Characterization of design kit's active device using an automated setup. Characterization includes DC characterization and high frequency behaviour specifically $f_{t}$ versus current density. Resulting measurements shall be used to help in establishing the useful region of operation and to verify the design kit's model.

\subsection{Organization}

The remainder of this thesis is organized into four chapters.

Chapter 2 presents the background material required for this thesis. GaN semiconductor material is presented. Its properties are discussed along with its conventional applications and a comparison to other semiconductor materials. The motivation to develop a good tuning mechanism in $\mathrm{GaN}$ is then presented with a discussion of switched tuning and varactors. Active inductors are then presented giving a detailed theory of operation along with a survey of existing research.

Chapter 3 concerns the design and simulation of an active inductor and tunable bandpass filter in GaN. Firstly the NRC GaN500 design kit is presented highlighting its features and limitations. The design goals are then presented for the active inductor and bandpass filter. The design of the active inductor is then presented including topology selection along with schematics and simulation results for each stage of the design. The bandpass filter design incorporating the active inductor is then presented along with the final layout for fabrication. 
Chapter 4 presents the measured results. The test setup is presented describing both the hardware and software components of the setup. The design kit's FETs are characterized for DC and $\mathrm{AC}$ behaviour. The active inductor is then characterized, and results are compared to simulation results obtained in Chapter 3. The bandpass filter measurements are then presented and again its results are compared to simulation.

Chapter 5 concludes the thesis. Updated simulation results are provided in an attempt to explain discrepancies between measured results of Chapter 4 and simulated results of Chapter 3. The contributions to research are summarized and suggestions for future work are provided. 


\section{Chapter 2}

\section{Background}

\subsection{GaN Semiconductor Material}

As wireless and mobile capabilities are packed more and more into various electronic devices, there is a push to both drive the cost down and miniaturize the technology. The trend is therefore towards highly miniaturized integrated circuits. This results in complete system on chip solutions where a single chip will provide full front-end transceiver capabilities and in some cases also baseband digital capabilities. Simultaneous to this drive to more fully integrated on chip solutions, is the need for devices with higher power handling capabilities and ability to operate in harsh environments.

Traditional semiconductor materials used to produce Monolithic Microwave Integrated Circuits include silicon, silicon carbide, gallium arsenide. These materials have low breakdown voltages and therefore have relatively low power handling capabilities.

Table 2.1 lists material properties of typical semiconductor materials used in high power RF applications [1].

One key property of semiconductor materials is the bandgap, $E_{g}$. The bandgap is the energy required to move an electron bound to an atom in the valence band to the conduction band. Once in the conduction band, the electron is free to move throughout the crystal lattice and carry charge thereby generating an electric current. 
TABle 2.1: Physical Properties of Semiconductors for High Power Devices

\begin{tabular}{|l|c||c|c|c|c|c|c|c|}
\hline Property & Units & $\mathrm{Si}$ & $6 \mathrm{H}-\mathrm{SiC}$ & $\mathrm{Ge}$ & $\mathrm{GaAs}$ & $\mathrm{GaP}$ & $\mathrm{InN}$ & $\mathrm{GaN}$ \\
\hline \hline$E_{g}$, Band Gap & $\mathrm{eV}$ & 1.1 & 3.0 & 0.66 & 1.4 & 2.3 & 1.9 & 3.4 \\
\hline $\begin{array}{l}n_{i}, \text { Undoped carrier } \\
\text { concentration }\end{array}$ & $\mathrm{cm}^{-3}$ & $1.5 \times 10^{10}$ & $2.3 \times 10^{-6}$ & $2.4^{*} 10^{13}$ & $1.8 \times 10^{6}$ & $7.7 \times 10^{-1}$ & $10^{3}$ & $1.9 \times 10^{-10}$ \\
\hline$E_{c}$, Critical Field & $M V / \mathrm{cm}$ & 0.3 & 2.4 & 0.1 & 0.4 & 1.3 & 1.0 & 3.3 \\
\hline$\mu_{n}$, Electron mobility & $\mathrm{cm}^{2} / V \cdot s$ & 1350 & 370 & 3900 & 8500 & 350 & 3000 & 900 \\
\hline $\begin{array}{l}\epsilon r, \text { Relative Permit- } \\
\text { tivity }\end{array}$ & - & 11.8 & 9.7 & 16.0 & 12.8 & 11.1 & 9.6 & 9.0 \\
\hline \begin{tabular}{l}
$\delta, \quad \begin{array}{l}\text { Thermal Conduc- } \\
\text { tivity }\end{array}$ \\
\hline $\begin{array}{l}v_{\text {sat }}, \text { Saturation Veloc- } \\
\text { ity }\end{array}$
\end{tabular} & $10^{7} \mathrm{~cm} / \mathrm{s}$ & 1.0 & 2.0 & 0.5 & 2.0 & 1.4 & 2.5 & 2.5 \\
\hline
\end{tabular}

Due to the increased required energy to enter the conduction band, semiconductors with wide band gaps have low intrinsic leakage currents adding to device efficiency.

Additionally, wide band gap materials perform more stably at higher temperatures[2]. The main mechanism for this is the dependence of a material's intrinsic carriers $n_{i}$ on both temperature and the band gap. This relationship is shown in Eq. 2.1

$$
n_{i}=\sqrt{N_{C} N_{V}} e^{-E_{G} / 2 k T}
$$

where $N_{C}$ and $N_{V}$ are the electron and hole densities of the material respectively, $T$ is the temperature in Kelvin, $k$ is the Boltzmann constant, and $E_{G}$ is the energy bandgap. Since $n_{i}$ increases with temperature, at a certain temperature, the concentration of intrinsic carriers will be on the same order as the concentration of dopant carriers making device behaviour uncertain. Since $n_{i}$ decreases with bandgap, this effect will not be as prevalent in wide bandgap materials at a given temperature than for a lower bandgap material such as $\mathrm{Si}$.

A second key property for semiconductors used in high power is the critical electric field $E_{c}$. The critical field is a useful parameter for identifying the onset of avalanche 
breakdown in power device structures[3]. The on-resistance of a power device is strongly related to the critical field. The relationship is shown in Eq. 2.2[4]

$$
R_{o n}=\frac{4 V_{B}^{2}}{\varepsilon \mu_{n} E_{c}^{3}}
$$

where $V_{B}$ denotes the device breakdown voltage, $\mu_{n}$ the device electron mobility, $\varepsilon$ the permittivity, and $E_{c}$ the critical field. A low $R_{o n}$ is critical for reducing device power dissipation and increasing device efficiency.

Although the on-resistance has a dependence upon the dielectric and the electron mobility, it is inversely proportional to $E_{c}$ cubed. A high $E_{c}$ therefore greatly reduces the device on resistance and device power dissipation.

Table 2.1 shows the critical field of GaN to be the highest of all materials at 3.3 compared to 0.4 for GaAs.

A third key property of a semiconductor material for high power applications is its thermal conductivity. A high thermal conductivity is desired to allow heat to disperse through a material allowing it to dissipate evenly thereby allowing the device to operate at higher power levels without incurring thermal damage.

Table 2.1 shows the thermal conductivity of GaN to be fairly high at $1.5 \mathrm{~W} / \mathrm{cm} \cdot \mathrm{K}$. Only silicon and silicon carbide at $4.5 \mathrm{~W} / \mathrm{cm} \cdot \mathrm{K}$ have a higher thermal conductivity than GaN.

The high thermal conductivity of silicon carbide is actually of significant interest to GaN devices. This is because GaN devices are most commonly formed upon a substrate of $\mathrm{SiC}$. The $\mathrm{SiC}$ substrate behaves like a heatsink for GaN devices pulling heat away from the active region of the die into the substrate material and ultimately onto a ground plane or thermal pad. 
The properties described above illustrate how $\mathrm{GaN}$ is an excellent candidate for high power applications. However, not only is GaN an attractive material for high power applications but its material properties also lend it well for high frequency applications.

A material's saturation velocity has a strong impact on a materials frequency of operation. Ideally, in the absence of any parasitics, a device's unity gain cut-off frequency can be expressed as [5]

$$
f_{t}=\frac{v_{s a t}}{2 \pi L_{g}}
$$

where $L_{g}$ is the device gate length. It is evident that for a fixed gate length, a higher saturation velocity results in a higher cut-off frequency.

Figure 2.1 shows how GaN positions itself against other semiconductor materials in regards to power and frequency performance.

GaN's performance at high frequency and high power has historically made it an obvious choice of material for RF / microwave power amplifier applications. Power amplifiers have been demonstrated at frequencies from the $\mathrm{X}$ and $\mathrm{K}$ bands[7][8] up into $\mathrm{E}$ and $\mathrm{W}$ bands[9][10].

Although GaN has typically been used in high power / high frequency applications it is now being used for applications not typical of GaN. LNAs have been demonstrated exhibiting noise figures below $2 \mathrm{~dB}$ [11][12]. Due to its inherent low on-resistance GaN makes excellent switches. This low on resistance potentially makes GaN an attractive candidate for other devices which require low series resistance such as varactors and active inductors. 


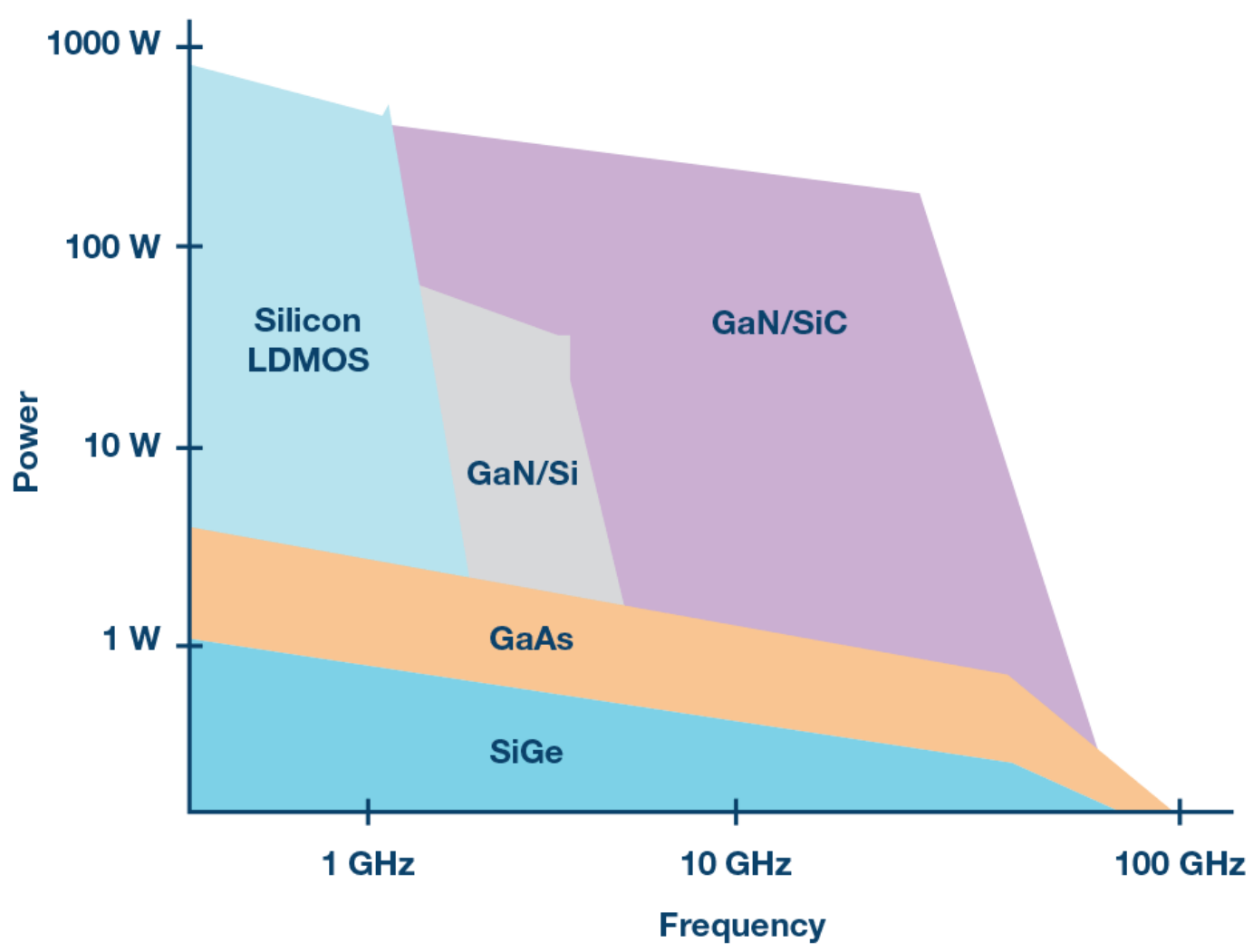

Figure 2.1: Performance of Semiconductor materials across frequency and power[6]

\subsection{Tunable Devices In GaN / MMIC}

To implement tunable circuits such as adaptable matching circuits, adaptable filters, and voltage controlled oscillators among others each circuit requires some form of tunable impedance mechanism.

Considering only devices that tune complex impedance i.e. reactance, the three most common mechanisms for tuning reactance are

- Switched reactance

- Varactors 
- Active Inductor

\subsubsection{Switched Reactance}

Switched reactances come in various topologies but the basic idea is to use switches to discretely change a circuit's reactance. The most common switched device is the digitally tuned capacitor (DTC). A typical example of a DTC is shown in Figure 2.2. As is shown, the overall capacitance of the device can be altered by opening or closing the individual switches which have a simple single pole single throw (SPST) topology.

Due to the discrete nature of the device, it is not an appropriate tuning mechanism for applications which require a device with a continuous tuning range such as the VCO in the feedback path of a PLL. DTCs are more typically used in adaptive impedance tuning / matching applications such as antenna matching for varying environments or changing the load impedance presented to a power amplifier thereby changing the PA's operating point and behaviour (e.g. gain, efficiency). Due to the high power involved in such applications highly linear devices are required. Along with high linearity the devices must exhibit a low on-resistance when in the 'on' state to minimize power losses and high isolation when the switch is in the 'off' state.

Historically RF switches have been implemented either using PIN diodes or GaAs FETs [13][14][15]. While the PIN diode has excellent switching characteristics and power handling capability it is not a common structure in typical MMIC processes and therefore does not lend itself to highly integrated designs. GaAs FET switches offer system on chip integration but do not typically have the power handling capability required [13] and may require the parallelizing of several devices thus increasing required area and complicating design [14]. GaN FETs therefore offer an excellent 
alternative to the traditional devices as they offer both high power handling and on chip integration. A drawback of implementing switch banks in GaN is that they require some amount of digital control / interface circuitry. Digital circuitry implemented in GaN is beginning to emerge as some new processes include both enhanced mode and depletion mode devices allowing for the construction of logic gates but is not mature at this stage[16][17].

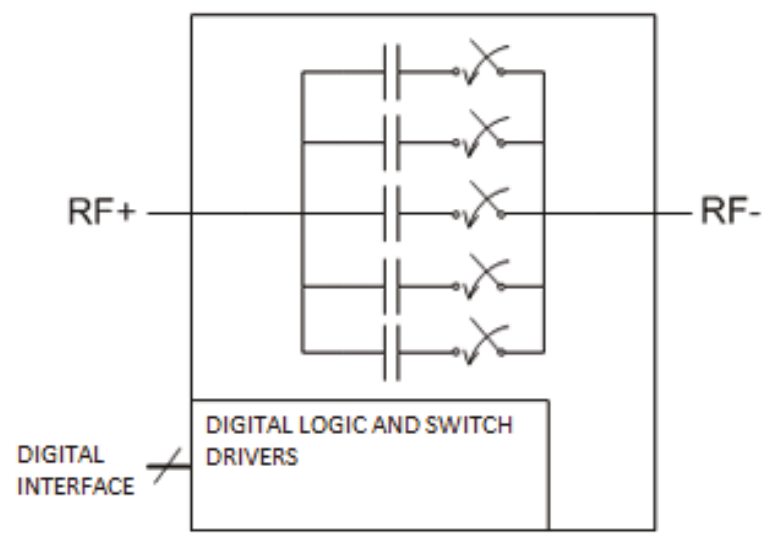

Figure 2.2: Typical example of digitally tuned capacitor[18]

\subsubsection{Varactors}

Varactors are the preferred device when continuous non-discrete impedance tuning is required. Varactors are the key building block within VCOs, phase shifters, adaptable filters among others.

In GaN / AlGaN processes, varactors are typically implemented using an MSM (metal semiconductor metal) topology or some variation thereof e.g. MSG - 2DEG. An MSM diode ideally consists of two Schottky diodes connected back to back, one in forward bias and the other in reverse bias [19]. The behaviour of the MSM-2DEG varactor under various bias conditions is illustrated in Figure 2.4 
MSM varactors can be implemented using planar topologies in GaN/AlGaN. A typical implementation is shown in Figure 2.3

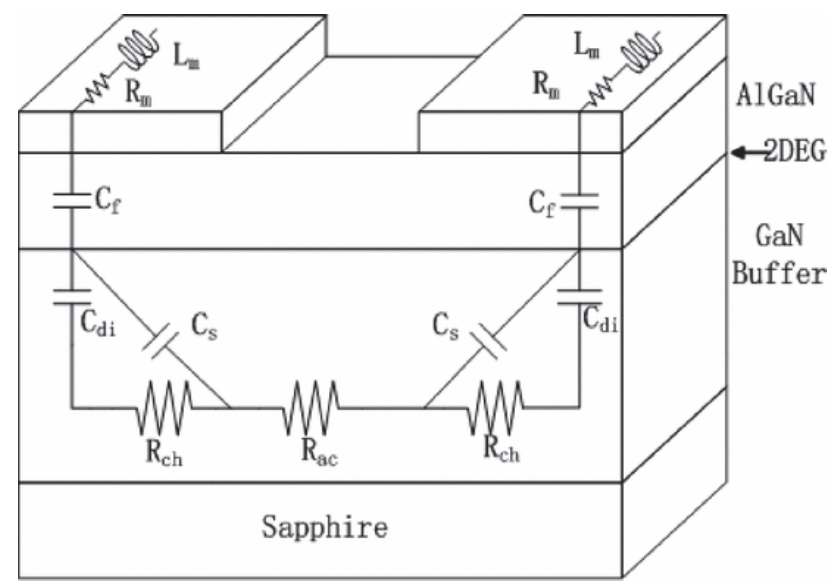

Figure 2.3: Typical GaN/AlGaN planar topology [20]



D)

Figure 2.4: Operation of the MSM-2DEG varactor a) unbiased device; b) VBIAS $=-\mathrm{V}_{\text {-th }}$; c) $\mathrm{V}_{\text {BIAS }}<<-\mathrm{V}_{\text {-th }}$; d) voltage / capacitance relationship [19]

With the NRC GaN500 design kit and similar GaN / AlGaN kits, GaN is undoped, making poor Schottky diodes with high series resistance. The result is varactors with relatively low quality factors. Examples of varactors implemented using the GaN500 process can be viewed in [21]. 
Several recent works have produced decent results in terms of quality factor but these works required the inclusion of specialized epitaxial layers.

In [22], a Q-factor of 35 at $1 \mathrm{GHz}$ was achieved however this required $\mathrm{n}+$ doping within the channel and also an n- layer below the metal contact. In [23] a device with a Q-Factor between 40 and 100 over the frequency range of 1 to $5 \mathrm{GHz}$ was published. However, this device again required special processing steps such as $\mathrm{n}+$ channel doping and n- layers beneath the contacts.

In [24], using the NRC GaN500 kit with no extra processing steps a varactor was presented. The varactor demonstrated favourable measured Q-factor at lower frequencies peaking at 150 at $1 \mathrm{GHz}$ but dropped off with frequency to 25 at $5 \mathrm{GHz}$. It demonstrated a favourable measured $\mathrm{C}_{\mathrm{MAX}} / \mathrm{C}_{\mathrm{MIN}}$ ratio of 2.4 with a midpoint capacitance around 400fF. However, this capacitance change occurred very rapidly over the range of $100 \mathrm{mV}$ thereby limiting the swing of the input RF signal and thereby providing low linearity.

In the absence of high quality factor varactors, and with switched impedance devices only capable of providing relatively coarse control over impedance, the active inductor presents an attractive alternative.

\subsection{Active Inductor Background}

Active inductors are synthetic devices that mimic the impedance - frequency relationship of a fixed inductor using active devices. The basis of most active inductors is the gyrator circuit. A detailed explanation of gyrator theory of operation is given in Section 2.3.1 
Active inductors provide several advantages over fixed inductors. Among the advantages are:

- tunable inductance

- low series resistance achievable

- reduction in area

- reduced electromagnetic radiation

Active inductors also have disadvantages compared to their fixed counterparts. Among them are:

- increased noise

- reduced linearity

- increased power consumption e.g. bias current

- potential stability issues

- more sensitive to process variations

- less bandwidth

It should be noted that although active inductors achieve much higher quality factors than fixed inductors, this comes at a significant cost; the cost being mainly additional power dissipation and noise. Whereas a fixed inductor aims to achieve a high quality factor by reducing losses caused by series resistance, an active inductor artificially creates low series resistances by injecting energy into the circuit to negate losses resulting in a higher power dissipation. Not only do the transistors dissipate extra 
power, but they also generate noise. The noise from the active transistors will always be higher than the thermal noise generated by the series resistance of a fixed inductor meaning the active inductor will always be noisier than a fixed inductor.

Active inductors can be single ended (i.e. one port is connected to ground, can only be used as shunt device) or differential devices (can be used as a series connected device). They come in various levels of complexity from fairly simple such as the single transistor Castro circuit to highly complex topologies.

\subsubsection{Active Inductor / Gyrator Theory of Operation}

The basis of most active inductors is the gyrator circuit [25] [26]. The ideal gyrator is shown in Figure 2.5

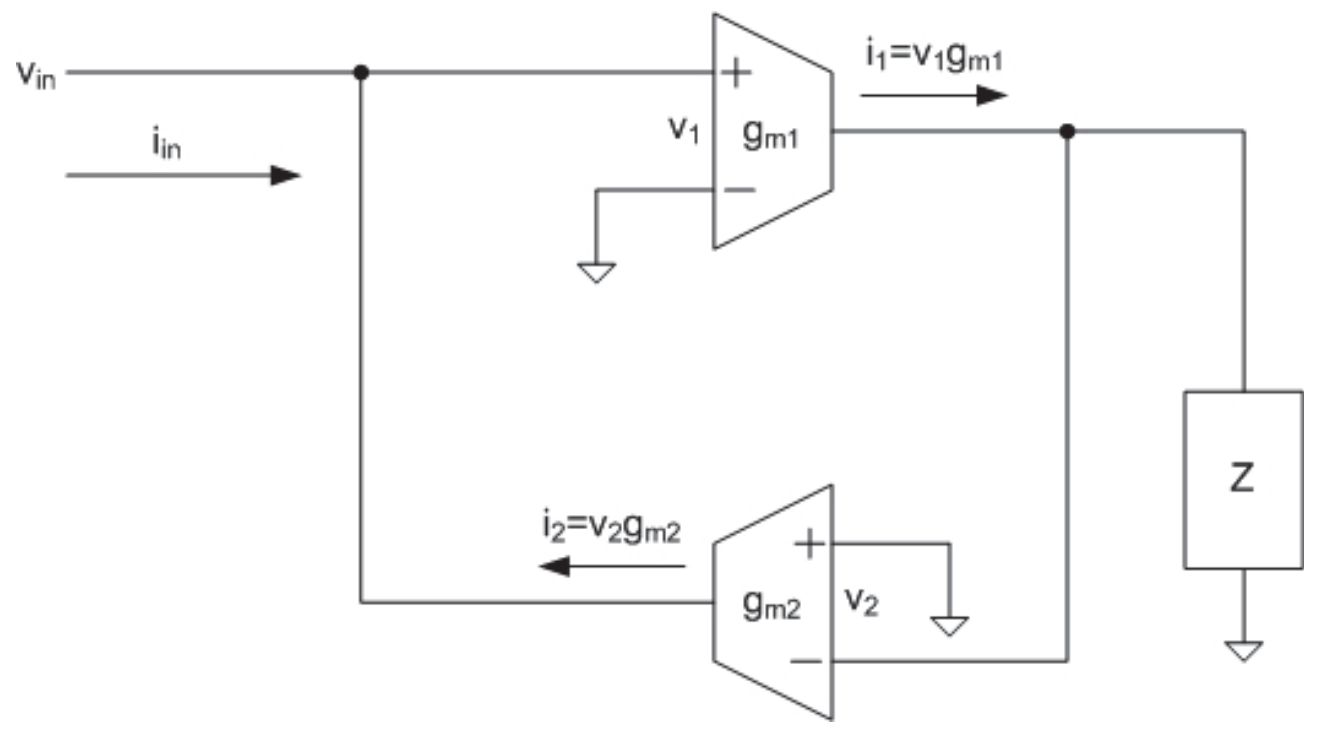

FiguRE 2.5: Block diagram of ideal gyrator

As shown, the gyrator consists of two transconductors, one positive and one negative, in a feedback configuration with some parallel impedance $\mathrm{Z}$ at the output of the 
first transconductor. Since the transconductors are ideal and have infinite input impedance, the input impedance of the gyrator can be found by basic circuit analysis. Transconductor 1 will produce an output current according to the differential voltage $v_{1}$ across its ' + ' and '-' terminals that is

$$
i_{1}=v_{1} g_{m 1}=v_{i n} g_{m 1}
$$

Since the second transconductor ideally has infinite input impedance, the voltage at the load $\mathrm{Z}$ is therefore

$$
v_{z}=i_{1} Z=v_{i n} g_{m 1} Z
$$

The output current of the second transconductor is again proportional to the differential impedance across its "+" and "-" terminals

$$
i_{2}=v_{2} g_{m 2}=-v_{z} g_{m 2}=-v_{i n} g_{m 1} g_{m 2} Z
$$

Since the first transconductor has infinite impedance and draws no current we have

$$
i_{\text {in }}=-i_{2}
$$

The input impedance, $Z_{\text {in }}$, is therefore

$$
Z_{i n}=\frac{v_{i n}}{i_{i n}}=\frac{v_{i n}}{-i_{2}}=
$$


Subbing in Eq. 2.6 to Eq. 2.8 gives

$$
Z_{i n}=\frac{1}{g_{m 1} g_{m 2} Z}
$$

The gyrator circuit therefore both inverts the impedance $\mathrm{Z}$ and also scales it by a factor of $\frac{1}{g_{m 1} g_{m 2}}$. When the impedance $\mathrm{Z}$ is formed by some ideal capacitance $\mathrm{C}$, such that

$$
Z=\frac{1}{s C}
$$

the input impedance is therefore equal to

$$
Z_{i n}=\frac{s C}{g_{m 1} g_{m 2}}
$$

The gyrator in this case, therefore, behaves as an inductor with an equivalent inductance of

$$
L_{E Q}=\frac{C}{g_{m 1} g_{m 2}}
$$

Each transconductor can be realized using one or more FETs in various configurations i.e. common gate, common source, and common drain. Typically the lumped capacitor $\mathrm{C}$ is implemented by the FETs' intrinsic capacitances. The inductance $L_{E Q}$ can be tuned by altering the DC bias conditions of the FETs which in turn alters the gm of each FET. When the ideal transconductors are replaced with real FETs, the model in Figure 2.5 is not fully accurate. A more accurate model is shown in Figure 2.6 


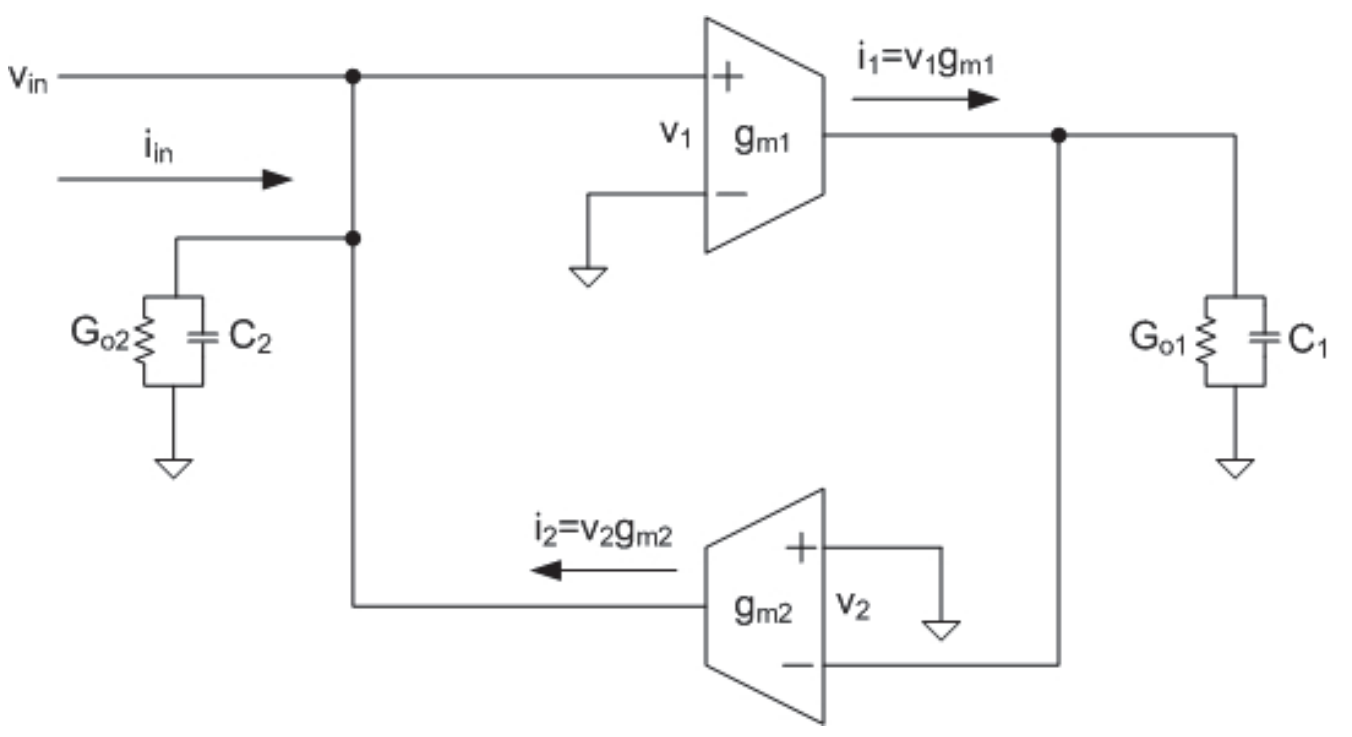

FiguRE 2.6: Block Diagram of Non Ideal Gyrator

The output of each transconductor now consists of a parallel conductance Go and parallel capacitance C. This model is still somewhat simplistic as it does not model the coupling between the input and output of each transconductor (e.g. $C_{G D}$ ) but it gives a general illustration of how the active inductor performance is degraded by parasitics.

Using the same circuit analysis as above, the input admittance Yin is obtained as

$$
Y i n=s C_{2}+G_{o 2}+\frac{1}{s \frac{C_{1}}{g_{m 1} g_{m 2}}+\frac{G_{o 1}}{g_{m 1} g_{m 2}}}
$$

This is represented schematically in Figure 2.7.

where $R_{p}=\frac{1}{G_{o 2}}, C_{p}=C_{2}, R_{s}=\frac{G_{o 1}}{\left(g_{m 1} g_{m 2}\right)}, L=\frac{C_{1}}{\left(g_{m 1} g_{m 2}\right)}$

As shown the inductance has been degraded by a series resistance $R_{s}$, a parallel capacitance $C_{p}$, and a parallel resistance $R_{p}$. 


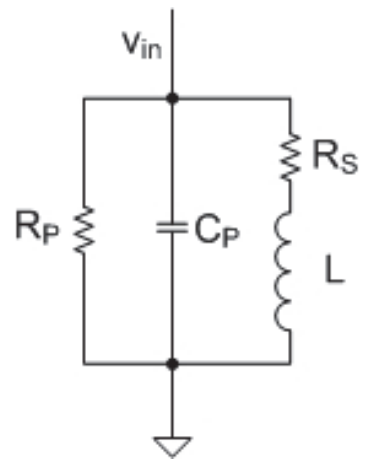

Figure 2.7: Equivalent Circuit Of Non Ideal Active Inductor

These parasitics degrade both the frequency performance of the inductor and the quality factor of the inductor defined as

$$
Q=\operatorname{mag}\left(\frac{\operatorname{imag}\left(Z_{i n}\right)}{\operatorname{real}\left(Z_{i n}\right.}\right)
$$

That is $\mathrm{Q}$ is the absolute ratio of the imaginary part of the complex impedance to the real part of the complex impedance. It is evident that in order to optimize the performance of the active inductor $R_{s}$ and $C_{P}$ must be minimized and $R_{P}$ must be maximized.

\subsubsection{Previous Works}

The published research concerning active inductors in MMIC in technologies other than CMOS is quite limited. There is some research using GaAs but the majority of it is fairly old. As far as the author knows, there is no published research concerning active inductors implemented in GaN. Furthermore, the bulk of recently published research into active inductors appears to be presented simulation results and fabricated device measurements. 
A summary of previous active inductor works is presented in Table 2.2. The application of each presented work varies from voltage controlled oscillator where the active inductor is used to produce a resonance at a desired frequency, low noise amplifiers where the active inductor is typically used to provide source degeneration and a wider matching bandwidth, and of particular interest to this work, in bandpass filters where the active inductor tunes the passband to a particular frequency. Unfortunately, some of the works only published the performance of the top level circuit (e.g. BPF) and did not publish any performance metrics of the active inductor thereby making comparison somewhat difficult.

TABle 2.2: Table of Previous Active Inductor Publications

\begin{tabular}{|c|c|c|c|c|c|}
\hline Ref. & Technology & $\begin{array}{l}\text { Frequency } \\
\text { Range } \\
(\mathrm{GHz})\end{array}$ & $\begin{array}{l}\text { Inductive } \\
\text { Range } \\
(\mathrm{nH}) \\
\end{array}$ & $\begin{array}{l}\text { Q Fac- } \\
\text { tor }\end{array}$ & Comments \\
\hline$[27]$ & $\begin{array}{l}0.2 \mathrm{um} \\
\text { GaAs } \\
\text { HEMT }\end{array}$ & 22 nominal & $0.87-0.97$ & 2000 & $\begin{array}{l}\text { Used to imple- } \\
\text { ment tuneable } \mathrm{BPF} \\
\text { from } 21.87 \mathrm{GHz} \text { to } \\
22.18 \mathrm{GHz}\end{array}$ \\
\hline$[28]$ & $\begin{array}{l}\text { 1um GaAs } \\
\text { MESFET }\end{array}$ & $\begin{array}{l}1.0 \text { nomi- } \\
\text { nal }\end{array}$ & 65-90 & 50 & $\begin{array}{l}\text { Inductance and Q } \\
\text { tuned by physically } \\
\text { changing external } \\
\text { capacitors }\end{array}$ \\
\hline
\end{tabular}




\begin{tabular}{|c|c|c|c|c|c|}
\hline$[29]$ & $\begin{array}{l}0.5 \mathrm{um} \\
\text { GaAs } \\
\text { pHEMT }\end{array}$ & $1.5-3.0$ & $1-5$ & 15 & $\begin{array}{l}\text { Used to implement } \\
\text { phaseshifter achieving } \\
180 \text { degrees of phase } \\
\text { shift }\end{array}$ \\
\hline [30] & $\begin{array}{l}0.180 \mathrm{um} \\
\text { CMOS }\end{array}$ & Not Stated & Not Stated & $\begin{array}{l}\text { Not } \\
\text { Stated }\end{array}$ & $\begin{array}{l}\text { Used to implement } \\
\text { tuneable BPF. BPF } \\
\text { achieved tuning range } \\
\text { of } 3.5 \text { to } 5.5 \mathrm{GHz} \text { with } \\
\text { a Q of } 665\end{array}$ \\
\hline$[31]$ & $\begin{array}{l}0.130 \mathrm{um} \\
\text { CMOS }\end{array}$ & Not Stated & Not Stated & $\begin{array}{l}\text { Not } \\
\text { Stated }\end{array}$ & $\begin{array}{l}\text { Used to implement } \\
\text { VCO with tuning } \\
\text { range of } 0.5 \mathrm{GHz} \text { to } \\
2.2 \mathrm{GHz}\end{array}$ \\
\hline$[32]$ & $\begin{array}{l}0.180 \mathrm{um} \\
\text { CMOS }\end{array}$ & Not Stated & Not Stated & $\begin{array}{l}\text { Not } \\
\text { Stated }\end{array}$ & $\begin{array}{l}\text { Used to implement } \\
\text { VCO with tuning } \\
\text { range of } 0.83 \mathrm{GHz} \text { to } \\
3.72 \mathrm{GHz}\end{array}$ \\
\hline [33] & $\begin{array}{l}0.180 \mathrm{um} \\
\text { CMOS }\end{array}$ & $0.006-6.30$ & $\begin{array}{l}5-550 \\
\text { (across } \\
\text { frequency, } \\
\text { not by } \\
\text { tuning) }\end{array}$ & 1070 & $\begin{array}{l}\text { Simulated only.Used } \\
\text { for LNA input match- } \\
\text { ing }\end{array}$ \\
\hline$[34]$ & $\begin{array}{l}0.180 u m \\
\text { CMOS }\end{array}$ & $0.6-2.4$ & $6.8-7.4$ & 1334 & Simulated Only \\
\hline
\end{tabular}




\begin{tabular}{|c|c|c|c|c|c|}
\hline$[35]$ & $\begin{array}{l}\text { 0.180um } \\
\text { CMOS }\end{array}$ & $1-5$ & Not Stated & $\begin{array}{l}\text { Not } \\
\text { Stated }\end{array}$ & $\begin{array}{l}\text { Simulated only. Used } \\
\text { to implement LNA } \\
\text { tuning circuit }\end{array}$ \\
\hline$[36]$ & $\begin{array}{l}\text { 45-nm } \\
\text { Predictive } \\
\text { Tech- } \\
\text { nology } \\
\text { MOSFET } \\
\text { Model } \\
\text { (Simu- } \\
\text { lation } \\
\text { Only) }\end{array}$ & $\begin{array}{l}2.5 \text { nomi- } \\
\text { nal }\end{array}$ & Not Stated & $\begin{array}{l}\text { Not } \\
\text { Stated }\end{array}$ & $\begin{array}{l}\text { Simulated only. Used } \\
\text { to implement fixed } \\
\text { BPF. Resonant fre- } \\
\text { quency of } 2.511 \mathrm{GHz} \\
\text { and bandwidth of } 57 \\
\mathrm{MHz} \text { simulated }\end{array}$ \\
\hline
\end{tabular}

\subsection{Summary}

In this chapter, the necessary background material for this thesis was presented. Firstly, standard semiconductor material properties such as band gap and critical field were presented and discussed. These properties were used as a basis to compare GaN to other competing semiconductor materials such as silicon and gallium arsenide. From this discussion, it was clearly shown that GaN is a promising semiconductor candidate for applications with requirements of both high power and high frequency.

Secondly a discussion of tuneable circuits and the means to achieve tuning in MMIC was presented. 
Switched components were presented highlighting their inherent limitations in achieving fine tuning due to their discrete nature.

Varactors were then presented as the main means of creating tuneable circuits in traditional semiconductor materials such as silicon. The inability to build good quality varactors in GaN due the high contact resistance was emphasized as being the core motivation to this thesis.

Active inductors were then presented as an alternative to varactors as a means of providing tuning in GaN MMICs. This was followed by an active inductor theory of operation demonstrating the gyrator and its behaviour in the presence of parasitics. Finally a literary survey of past research of active inductors in MMICs.

Chapter 3 will build upon the general GaN background material presented in this chapter by introducing the GaN500 design kit. The will be followed by the presentation of the design and simulation of an active inductor and tunable bandpass filter using the above-mentioned design kit. 


\section{Chapter 3}

\section{Design}

\subsection{Gan500 Design Kit}

The Canadian Photonics Fabrication Center (CPF) at the National Research Council Canada (NRC) offers a foundry service for AlGaN/GaN HEMT based MMICs. In particular, the GaN500 process was used for this research which features a gate length of $0.5 \mathrm{um}$. The time-line of this research spanned several revisions of the GaN500 design kit, but the final manufactured design was performed using revision 3.0 [37].

Using the process described in [37], GaN HFETs are fabricated on 3-inch diameter epitaxial layers formed by AlGaN/GaN layers, grown on insulating silicon carbide wafers of 75 um finished thickness. The resulting active component is an N-type depletion mode HFET, with a gate length of 0.5 um and varying gate widths.

A cross section of the resulting HFET structure is shown in Figure 3.1[37]. The layers are described below.

- SiC substrate- The substrate provides a mechanical support and acts as a heatsink drawing heat away from the active region

- Nucleation- This layer is used to allow the growth of GaN on top of the SiC substrate by matching the GaN lattice structure to that of the substrate

- Gan Buffer- This layer restricts the movement of electrons to within the channel and prevents electrons from drifting into the substrate 
- GaN- This layer is the active channel of the device

- AlN Spacer- This spacer helps with the 2-dimensional gas concentration and mobility[38]

- AlGaN- This layer acts as a barrier between the gate and the channel and helps reduce the gate leakage current into channel [39]

- GaN Cap This layer is added for several reasons such as reducing gate leakage current, increasing breakdown voltage[40]

- Contacts- Ohmic contacts are used for the HFET junctions

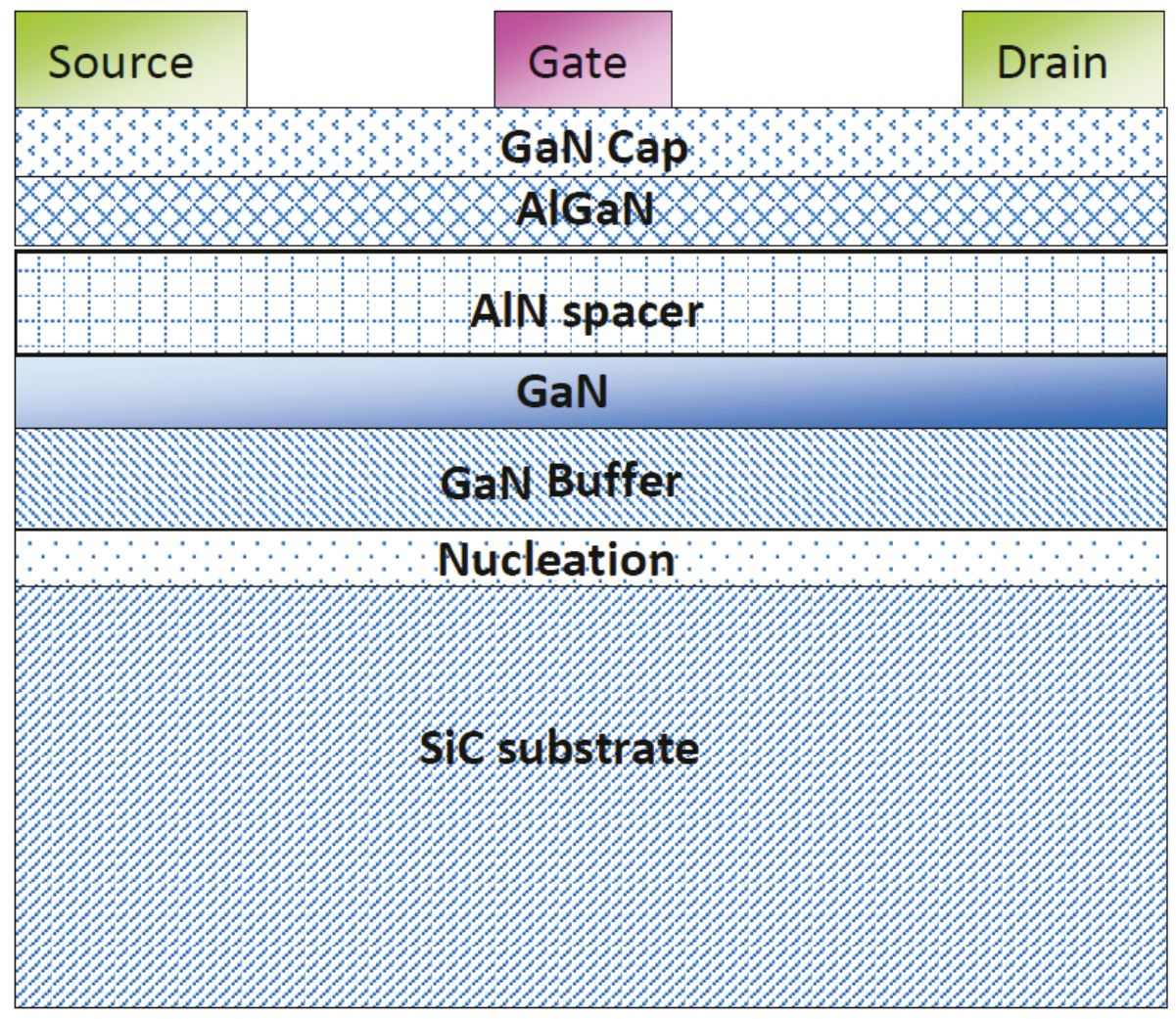

FiguRE 3.1: NRC GaN500 HFET Structure

Devices are specified as having nominal cutoff frequency $f_{t}$ of $13 \mathrm{GHz}$ and maximum frequency of oscillation $f_{\max }$ of $60 \mathrm{GHz}$. The HFET's maximum current density is 
TABLE 3.1: GaN500 Interconnect Properties

\begin{tabular}{|c|c|c|c|}
\hline Interconnect Layer & $\begin{array}{c}\text { Resistivity } \\
(\mathbf{m O h m} / \mathbf{s q})\end{array}$ & $\begin{array}{c}\text { Max. Current } \\
(\mathbf{m A} / \mathbf{u m} \text { width })\end{array}$ & $\begin{array}{c}\text { Layer Thickness } \\
(\mathbf{u m})\end{array}$ \\
\hline 1ME & 27 & 6 & 1 \\
\hline 2ME & 9 & 18 & 3 \\
\hline 1ME+VIA2+2ME & 6.75 & 24 & 4 \\
\hline
\end{tabular}

specified as $J_{D S S}=1 \mathrm{~A} / \mathrm{mm}$ and its maximum transconductance is specified as 0.24 $\mathrm{S} / \mathrm{mm}$.

In addition to the active HFET, the process offers several passive structures for design of MMICs.

Two interconnect layers, $1 \mathrm{ME}$ and $2 \mathrm{ME}$ are implemented in gold. The electrical properties of which are summarized in Table 3.1

Capacitors are formed using a Metal-Insulator-Metal (MIM) topology. Specifically, capacitors are formed with $2 \mathrm{ME}$ on top of $1 \mathrm{ME}$ with a $0.3 \mathrm{um}$ layer of silicon nitride in between. The capacitance density of the structure is $0.19 \mathrm{fF} / \mathrm{um}^{2}$ allowing a maximum capacitance of $122 \mathrm{pF}$. The minimum dielectric breakdown of the structure is $180 \mathrm{~V}$ allowing a maximum recommended operating voltage of $40 \mathrm{~V}$.

Resistors are formed using a thin layer of Nichrome(NiCr) with contacts to $1 \mathrm{ME}$. The resulting resistors have a resistivity of $50 \mathrm{Ohm} / \mathrm{sq}$ with tolerances better than $10 \%$.

Fixed inductors are formed using a square spiral topology wound around a central empty area. The inductors turns are formed using $1 \mathrm{ME}$ and $2 \mathrm{ME}$ combined together forming a low resistance conductor and thereby increasing the $\mathrm{Q}$ factor of the inductor. 


\subsection{Design Goals}

An active inductor shall be designed and produced using The National Research Center's GaN500 process. Initially, the device was required to operate with a nominal operating frequency of $10 \mathrm{GHz}$. However, changes to the GaN500 design kit and a major reduction in device transit frequency forced a change in the frequency of operation to $5 \mathrm{GHz}$. An inductance on the order of $1 \mathrm{nH}$ was to be aimed for. Values on the order of $1 \mathrm{nH}$ are fairly typical in and around $5 \mathrm{GHz}$. Also noting that $1 \mathrm{nH}$ resonates with $1 \mathrm{pF}$ at $5 \mathrm{GHz}$, and capacitors of $1 \mathrm{pF}$ are very practical to implement with the GaN500 design kit at an operating frequency of $5 \mathrm{GHz}$, the chosen value of $1 \mathrm{nH}$ is quite also quite practical.

Once the active inductor is designed it shall be incorporated into a bandpass filter. Again a nominal operating frequency of $5 \mathrm{GHz}$ shall be aimed for.

Both circuits, the stand-alone active inductor and the bandpass filter incorporating the active inductor shall be fabricated on a $2 \mathrm{~mm} X 2 \mathrm{~mm}$ die as it is a standard die size for the GaN500 process.

\subsection{1 $\quad F_{t}$ and Selection of Operating Frequency}

At this point is worth discussing the choice of operating frequency in consideration of a

processes' $f_{t} . F_{t}$ is a figure of merit regarding the speed of a device. Essentially it is the frequency at which the device's forward gain $\mathrm{h}_{21}$ is equal to 1 . There are other figures of merit in regard to the speed of a device such as $f_{\max }$ which specifies the maximum frequency at which the device can produce forward gain. $\mathrm{F}_{\max }$ is not a particularly useful figure of merit for the case of an active inductor in which power transfer is not a concern but rather the transfer of signal amplitude in the form of voltage or current is 
of concern. In this case $\mathrm{f}_{\mathrm{t}}$ is a more relevant figure of merit. Although $\mathrm{f}_{\mathrm{t}}$ indicates the point of maximum forward current gain it does not directly indicate the maximum typical operating frequency of a device. There is no literature relating a device's $\mathrm{f}_{\mathrm{t}}$ to a recommended operating frequency. This is probably because it varies from application to application and is therefore somewhat subjective. Various numbers are stated in web articles relating design frequency to ft such as being anywhere between $1 / 20$ to $1 / 5$. However these numbers are unsubstantiated and are very much "rule of thumb". Therefore, to get an understanding of the useful operating range of a device given $f_{t}$, we must consider the device's function in the circuit and also how $f_{t}$ relates to a device's physical parameters.

In an active inductor circuit, the FETs should behave as ideal transconductors (voltage controlled current source). The input to the device's gate should be high impedance so that it just not pull down the source that is driving it. Should the input have a low impedance the input voltage will be pulled down, and the FET output current will also be lowered since the drain-source current is directly related to the gate-source voltage by $g_{m}$. The input impedance to the gate is predominantly determined by $\mathrm{C}_{\mathrm{in}}$ which is the sum of the gate-source and gate-drain capacitances $\mathrm{C}_{\mathrm{GS}}$ and $\mathrm{C}_{\mathrm{GD}}$ respectively

Ft is bias dependent and is commonly related to the device's input capacitance $\mathrm{C}_{\text {in }}$ and transconductance $g_{m}$.

$$
f_{t}=\frac{g_{m}}{2 \pi C_{i n}}
$$

For the GaN500v3.0, $\mathrm{f}_{\mathrm{t}}$ is nominally specified as being $13 \mathrm{GHz}$, whereas $\mathrm{g}_{\mathrm{m}}$ for a 50um wide device is specified as being nominally $0.012 \mathrm{~S}$. 
This equates to a nominal $\mathrm{C}_{\text {in }}$ of $0.15 \mathrm{pF}$. At a frequency of $\mathrm{f}_{\mathrm{t}}$ the input impedance corresponding to $\mathrm{C}_{\text {in }}$ will be $83 \Omega$ or $1 / \mathrm{g}_{\mathrm{m}}$. This is a fairly low input impedance and would pull down any voltage source unless it had a very low series output impedance. The operating frequency should therefore be backed off. Using the " $1 / 10 f_{t}$ " rule of thumb, this results in an operating frequency of $1.3 \mathrm{GHz}$ and an input impedance of magnitude $830 \Omega$. This is a much higher gate input impedance and will pull down the voltage at the gate to a much smaller extent representing a more ideal transconductor.

In this application, a frequency of operation of less than $1 / 3 f_{t}$ was selected. This is a very aggressive design goal and presents considerable risk. However, it should be noted that at the time of specifying the design requirements, there was considerable interest in pushing GaN to operate at multi-GHz frequencies which is why the decision of such a high operating frequency was chosen.

\subsection{General Design Considerations}

This section outlines some general design considerations and constraints to be considered for the active inductor design when using the GaN500 design kit.

\subsubsection{High Frequency Operation and Design Kit Limitations}

Although there are many active inductors topologies to choose from, the number of topologies that can be implemented using the GaN500 design kit is limited.

Firstly, the GAN500 design kit only contains N-type FETs, therefore no topologies using P-Type FETs could be implemented. 
Secondly, only single ended active inductor topologies were considered. That is, only topologies where one port of the AI is referenced to ground were considered. The reasons for this were several-fold:

- Balanced topologies can take double the space. On a $2 \mathrm{~mm}$ X $2 \mathrm{~mm}$ chip, space is very limited

- In balanced topologies, both sides of the circuit must match each other very closely. It is hard to guarantee this behaviour using a non-mature design kit such as the GaN500.

Secondly, due to the high frequency operation, the topology should be as simple as possible to limit transmission line and coupling effects that are more prevalent at high frequencies. Therefore, the number of FETs, passive devices, and interconnect complexity should be all be minimized.

\subsection{Active Inductor Topology Selection}

After some initial consideration of various topologies and considering the constraints imposed by the design kit, two main topologies were considered for the active inductor:

- Common Source - Common Gate Topology

- Common Source - Common Drain Topology

Each topology presented and discussed below. 


\subsubsection{Common Source - Common Gate Topology}

The basic Common Source - Common Gate Topology is illustrated in Figure 3.2. As an aside, it is noted that the design kit's HFETs have two source connections however only one connection is used. The two source connections are connected physically on the device but are supplied for power / heat dissipation purposes. However, since this is not a high power application, only a single source connection is used to simplify layout.

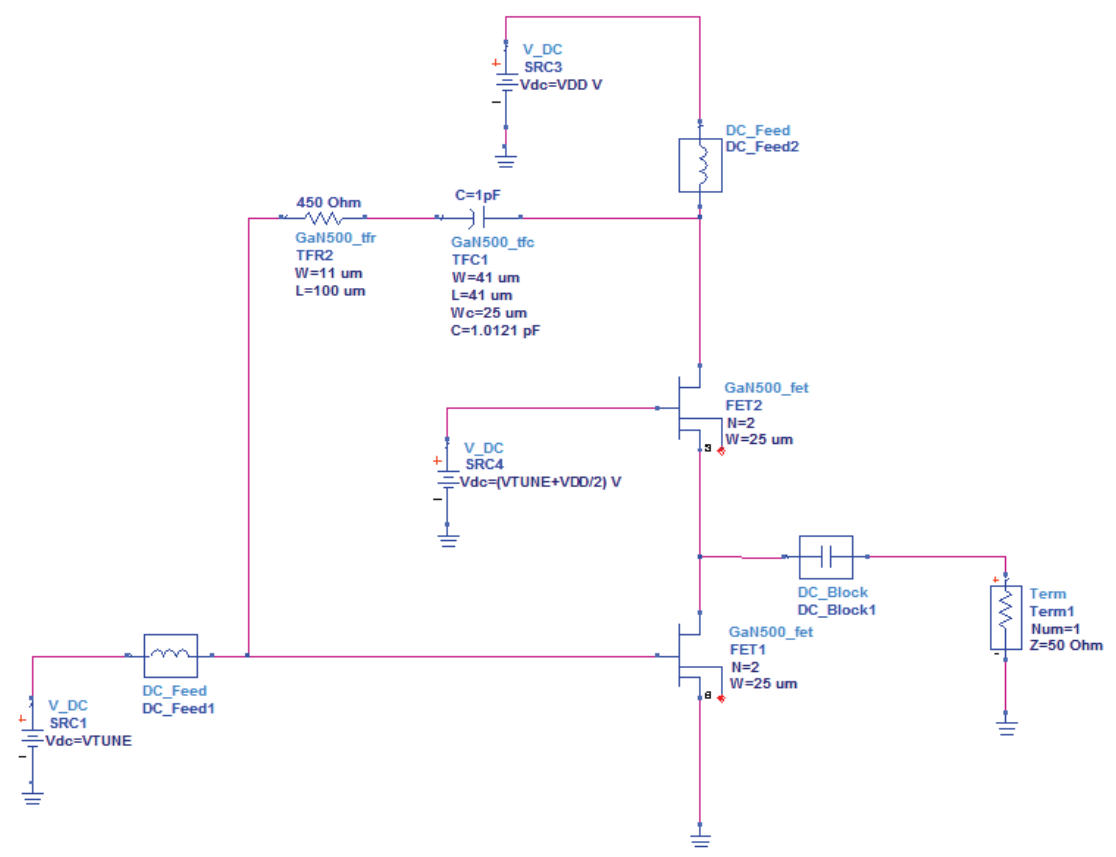

Figure 3.2: Basic common source-common gate topology

This topology is extremely similar to a cascode amplifier topology. The bottom transistor is configured in common-source configuration with its drain connected to the second transistor's source which is in common-base configuration. The output of FET2 (drain) is then connected to the input (gate) of FET1 completing the feedback path. This node also serves as the single ended input / output port to the device. 
Advantages of this topology are:

- Both FETs are in series and therefore share the same bias current $I_{D S}$ thereby potential reducing power consumption for a fixed VDD

- Only a single biasing component required since only one $I_{D S}$ is required (not counting gate biasing resistors)

Potential disadvantages of this topology are:

- Since both transistors share the same $I_{D S}$ they shall be biased similarly, therefore there are limited degrees of freedom in tuning the circuit

A full layout and EM-schematic of this topology was performed using the GaN500 v1.5 design kit. The final layout of which is shown in Figure 3.3.

The resulting behaviour versus frequency is shown in Figure 3.4.

This active inductor demonstrates an inductance of $0.5 \mathrm{nH}$ at $9.5 \mathrm{GHz}$ increasing to $2.6 \mathrm{nH}$ at $10.5 \mathrm{GHz}$. The $\mathrm{Q}$ factor for this device is fairly poor and peaks at 20 at 10.3 GHz. For this reason an alternative topology was explored. 


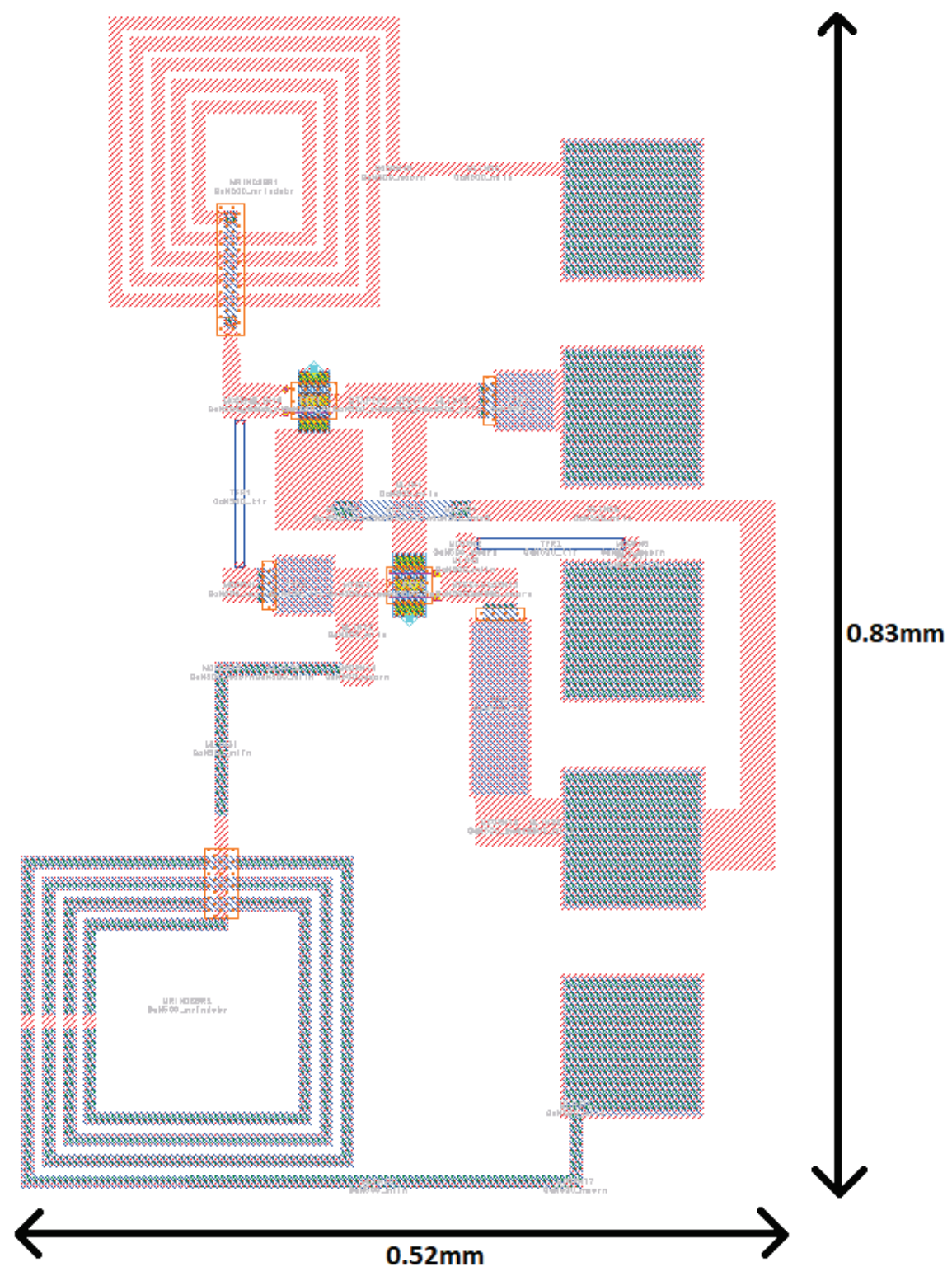

Figure 3.3: Common source-common gate layout 


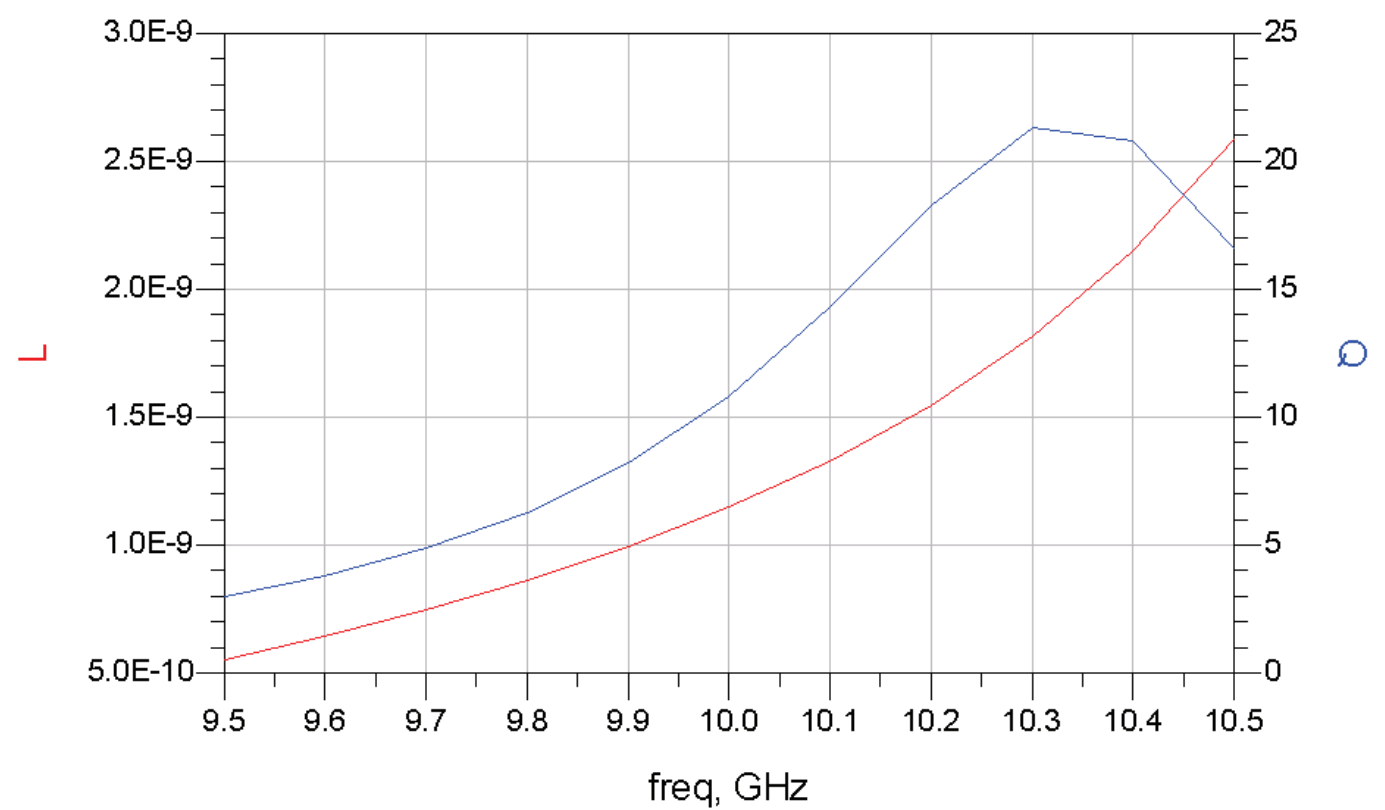

Figure 3.4: Common source-common gate Simulated Frequency Performance

\subsubsection{Common Source - Common Drain Topology}

The basic topology of the Common Source - Common Drain topology is shown in Figure 3.5 along with idealized DC feeds and DC blocks.

FET1 is the input device in common source configuration. Its drain output is connected to the gate input of FET2 which is in a common drain configuration. The output of FET2 then connects to the input of FET1 creating the necessary feedback path for a gyrator.

Advantages of this topology are:

- Potential to use a lower VDD supply since FETs are in parallel and have more headroom 


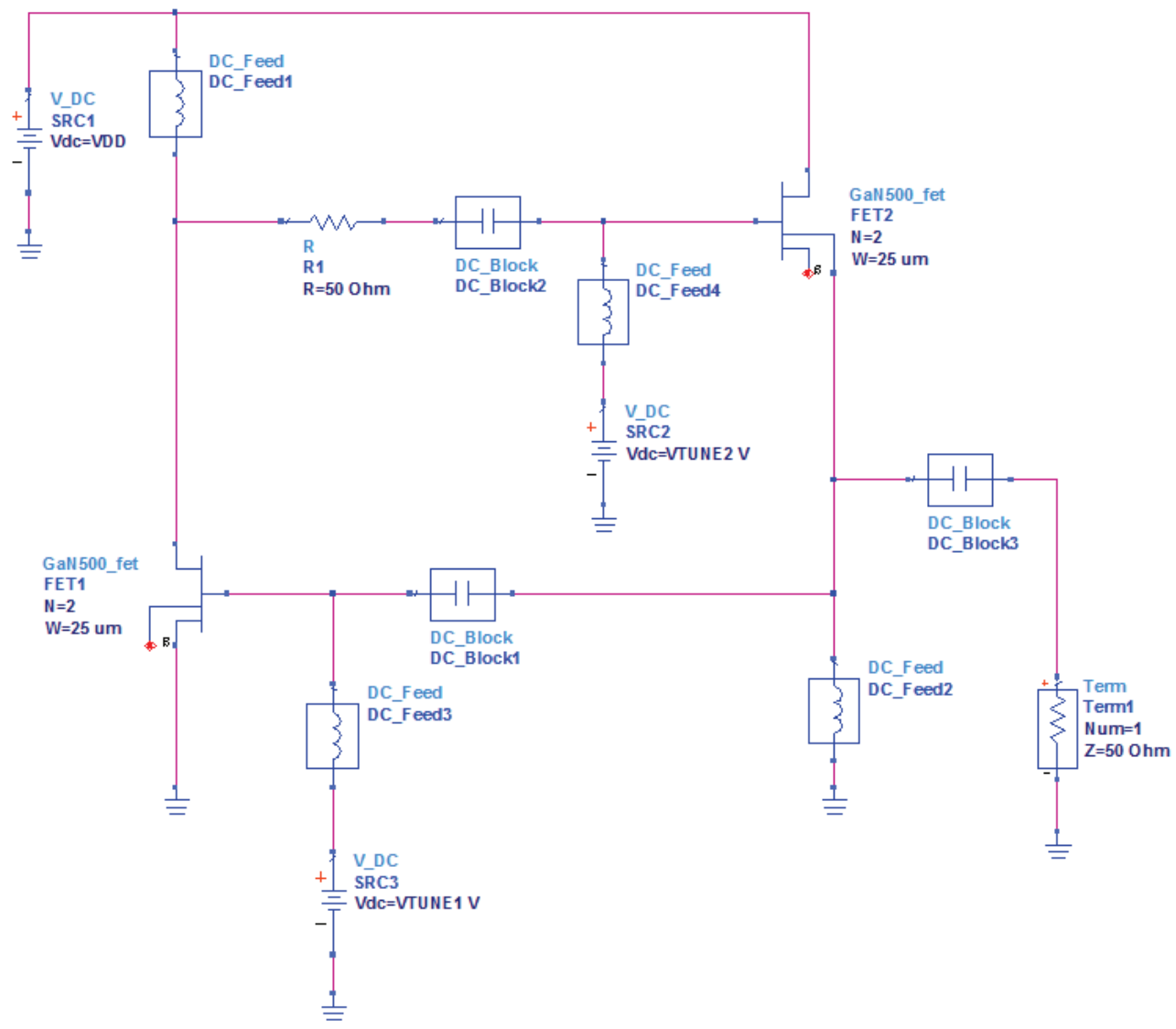

FiguRE 3.5: Basic common source-common drain topology

- Since FETs are in parallel and each has independent $I_{D S}$, the circuit has more degrees of freedom for tuning

Potential disadvantages of this topology are:

- Each FET draws independent $I_{D S}$ increasing power consumption for a fixed VDD

The advantages and disadvantages of the two considered topologies are summarized in Table 3.2 
TABle 3.2: Active Inductor Topology Comparison

\begin{tabular}{|l|l|l|}
\cline { 2 - 3 } \multicolumn{1}{c|}{} & \multicolumn{2}{c|}{ Topology } \\
Feature & $\begin{array}{l}\text { Common Source - } \\
\text { Common Gate }\end{array}$ & $\begin{array}{l}\text { Common Source - } \\
\text { Common Drain }\end{array}$ \\
\hline \hline Drain Current & Shared Between Devices & 2X Drain Current \\
\hline VDD Supply & $\begin{array}{l}\text { Requires headroom for } \\
\text { two VDS drops }\end{array}$ & Single VDS drop \\
\hline Biasing Devices & 1 & 2 \\
\hline $\begin{array}{l}\text { Number of Tuning } \\
\text { Mechanisms }\end{array}$ & 1 & 2 \\
\hline
\end{tabular}

The devices are more or less even on power consumption since the Common Source

- Common Gate requires double the VDD but half the current than the Common Source - Common Drain. Common Source - Common Gate only required a single biasing component. However, the deciding factor was that the Common Source Common Drain has more degrees of tuning.

\subsection{Active Inductor Design}

The idealized Common Source - Common Drain topology presented in 3.5 was simulated. The simulation results across frequency are shown in Figure 3.6. As is evident, the device shows a decent inductance at $1 \mathrm{GHz}$ of $1.1 \mathrm{nH}$. The device has a resonance close to $9 \mathrm{GHz}$ where the peak inductance is $1.45 \mathrm{nH}$ representing an approximate $30 \%$ increase in inductance across a $900 \%$ increase in frequency. The $\mathrm{Q}$ factor however is quite poor and peaks around 6. Adjusting the tuning voltages VTUNE1 and VTUNE2 had minimal effect on the Q factor.

To improve the behaviour of the device, the equivalent circuit of a basic gyrator circuit presented in Figure 2.7 is recalled. In this model, the inductance has a series resistance $R_{S}$. This series resistance is largely proportional to the conductance of 


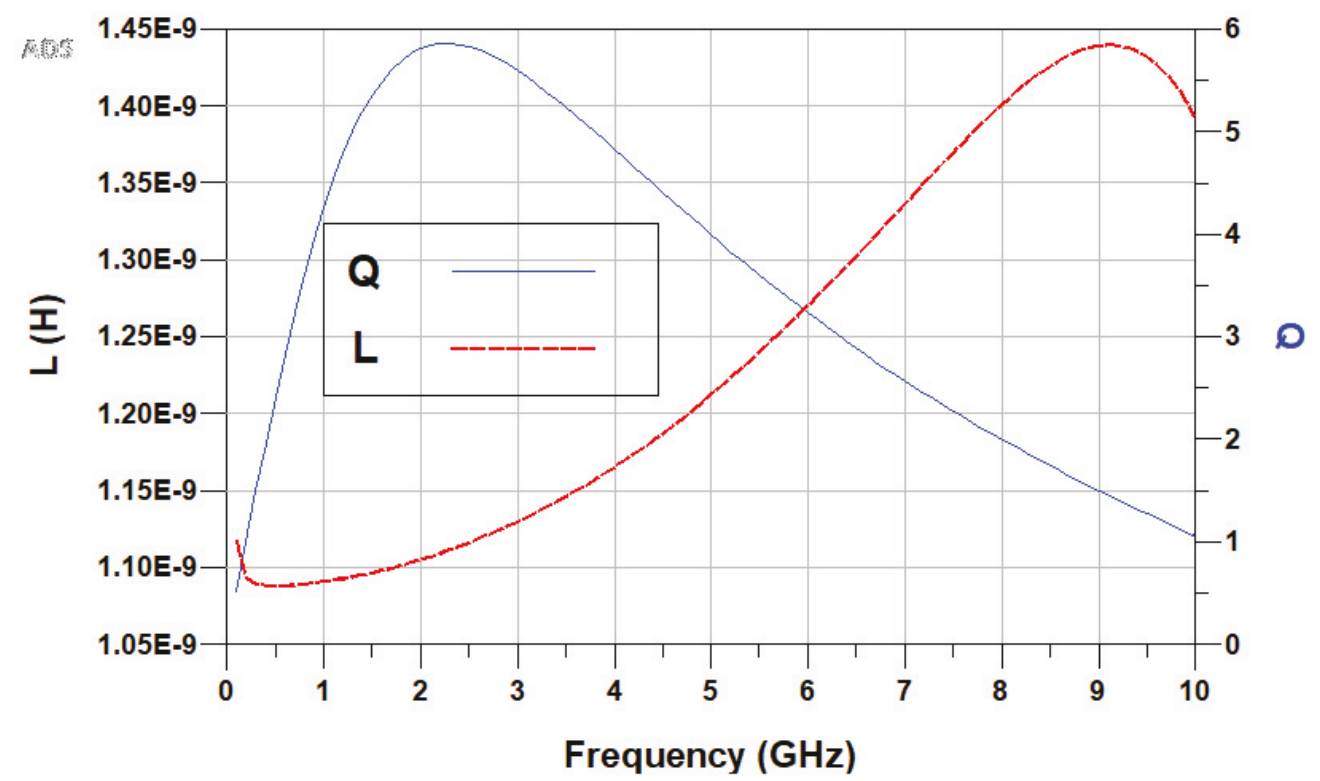

FigURE 3.6: Basic common source-common drain simulated results

the input transconductor e.g. FET1 in this case. Reducing the conductance of this FET (or equivalently increasing the FET's output impedance) will in turn reduce the series resistance of the active inductor and will thereby increase the device's $Q$ factor.

One method of increasing the FET1's output resistance is to transform the single common source configuration into a cascode configuration. Adding a second FET in common base configuration will act as a current driver increasing the output resistance of the front end transducer thereby reducing the series resistance of the active inductor.

The active inductor with FET1 transformed into a cascode pair of FET1 and FET3 is shown in Figure 3.7.

The simulated inductance and Q factor are shown in Figure 3.8. 


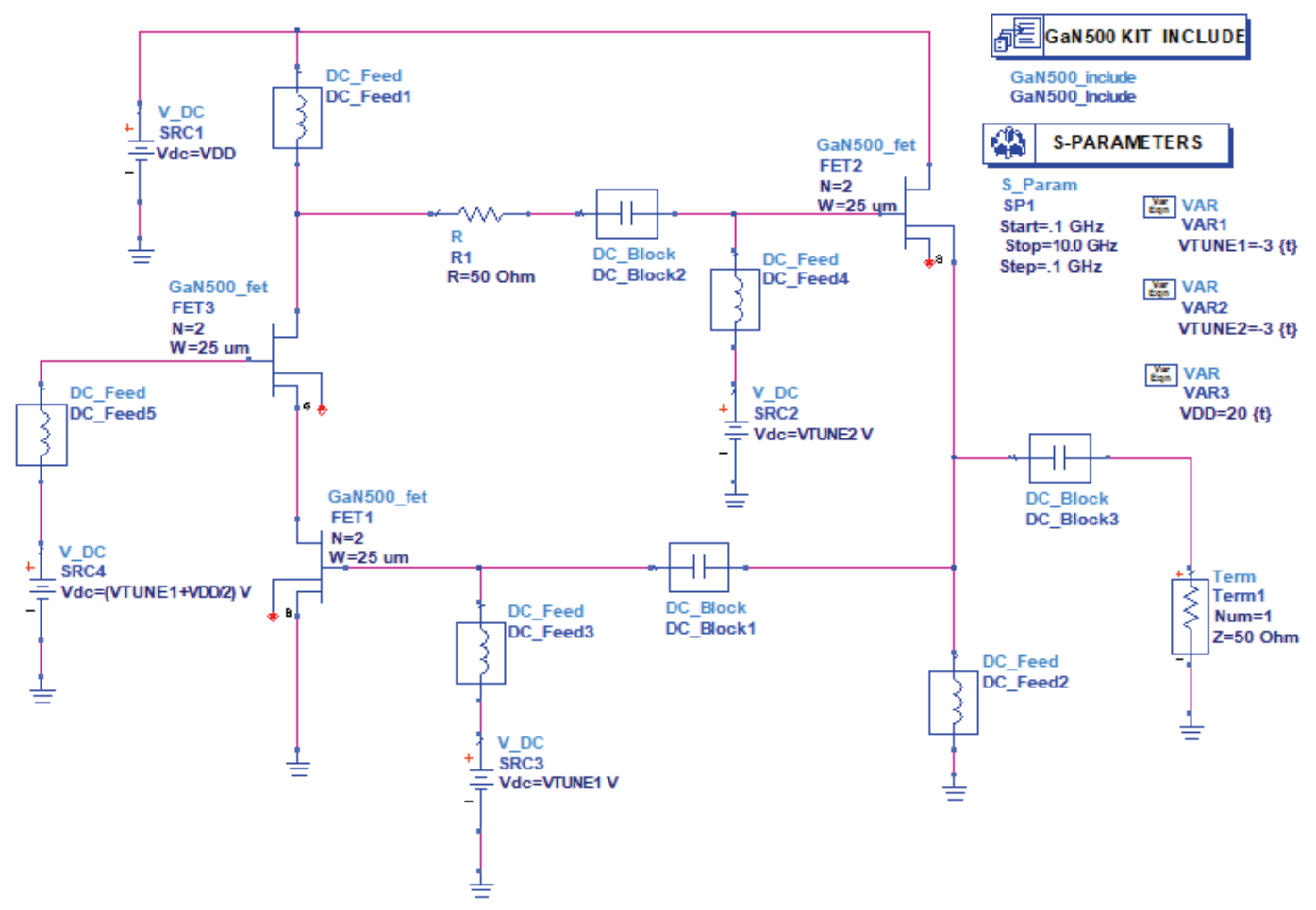

Figure 3.7: Cascode Amplifier - common drain topology

The device exhibits a simulated inductance of $1.4 \mathrm{nH}$ at $1 \mathrm{GHz}$ increasing to $2.8 \mathrm{nH}$ around $7 \mathrm{GHz}$ at resonance.

The $\mathrm{Q}$ factor has improved to a peak of approximately 16 at $3.2 \mathrm{GHz}$ and demonstrates a Q factor greater than 10 over a frequency ranging from $2.0 \mathrm{GHz}$ to 4.4 GHz.

The next step in the design was to replace the ideal biasing components (i.e. DC blocks, and DC feeds) with real biasing components. DC blocks components were replaced with capacitors with nominal values of $1 \mathrm{pF}$. DC feeds on the gates were replaced with large resistors whereas DC feeds on the source or drains were replaced with fixed inductors from the GaN design kit to minimize IR voltage drops. The inductor components from the design kit simulate parasitic behaviour such as series 


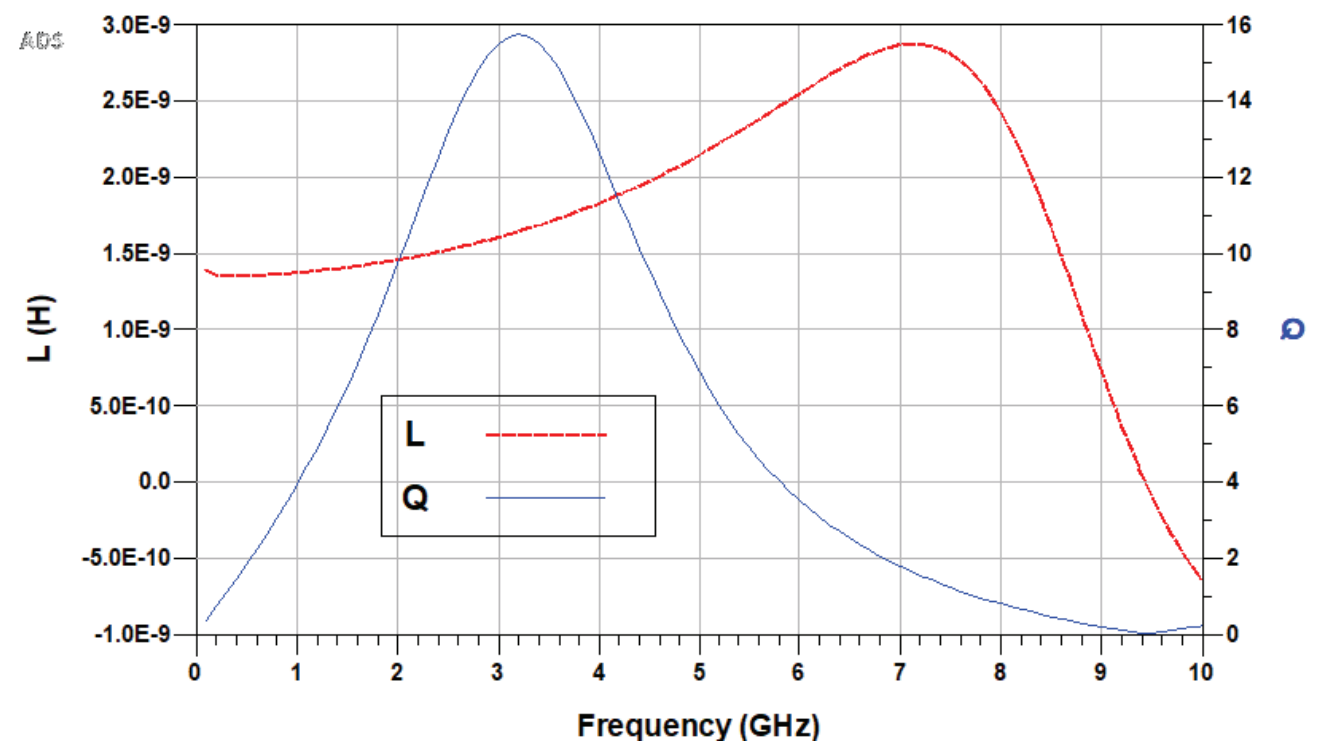

Figure 3.8: Cascode Amplifier - common drain simulated results

resistance and self-resonance. The model behaviour is also very close to the model behaviour of the behaviour obtained from EM simulation.

At this point it should be noted explicitly that fixed inductors are used to bias the circuit. On the surface, it seems counterproductive to design an active inductor only to use traditional fixed inductors within the design. The motivation of this thesis is therefore emphasized which is to build a tunable inductor since quality varactors cannot be fabricated in GaN. Where possible resistors or active biasing was used but due to limitations in the design kit (i.e. only depletion mode n-type HFETs), this was not always possible. Other biasing techniques could have been used such as quarter wave elements or radial stubs but these components occupy just as much if not more space than an inductor at the frequency of interest and are band limited.

The schematic with real biasing components is shown in Figure 3.9 and its corresponding results are shown in Figure 3.10. 
Two noticeable behaviour changes can be observed from the introduction of the real biasing circuitry. Firstly, the inductance has become notably frequency dependent.

The device is capacitive below $4.4 \mathrm{GHz}$ and increases in inductance until self-resonance at $6.4 \mathrm{GHz}$ where it exhibits peak inductance of $14 \mathrm{nH}$. The device demonstrated a decent $\mathrm{Q}$ of 60 around 5.2GHz.

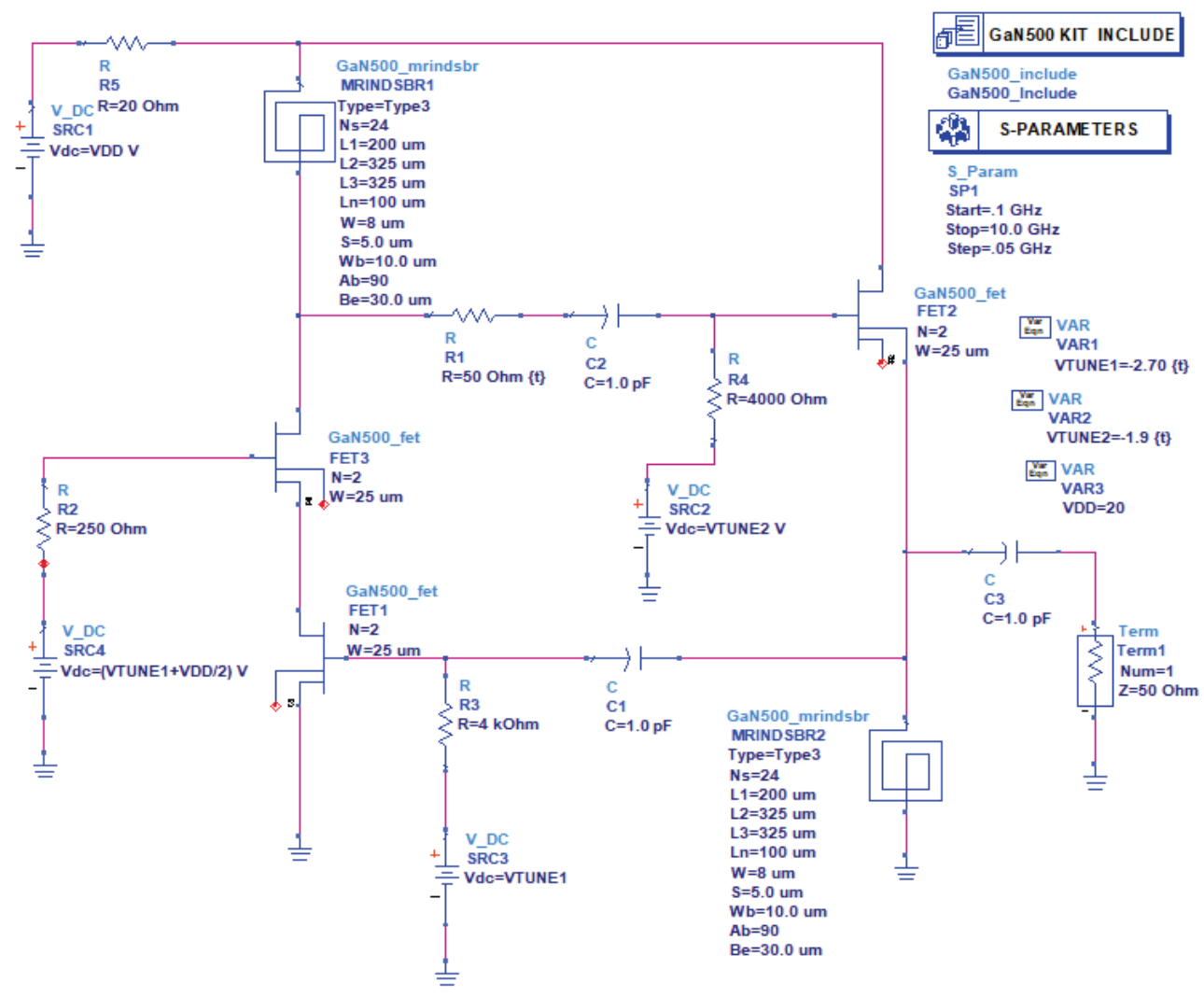

FiguRE 3.9: Schematic with real biasing components

The next step was the replacement of the bias inductor for FET2 with active biasing transistor FET4. The resulting schematic and simulation are shown in Figure 3.11 and Figure 3.12 respectively. The noticeable change in the simulation result is the $\mathrm{Q}$ factor. At $5 \mathrm{GHz}$, the $\mathrm{Q}$ factor is now around 150 and slightly below at $4.9 \mathrm{GHz}$ the Q factor is above 500 . 


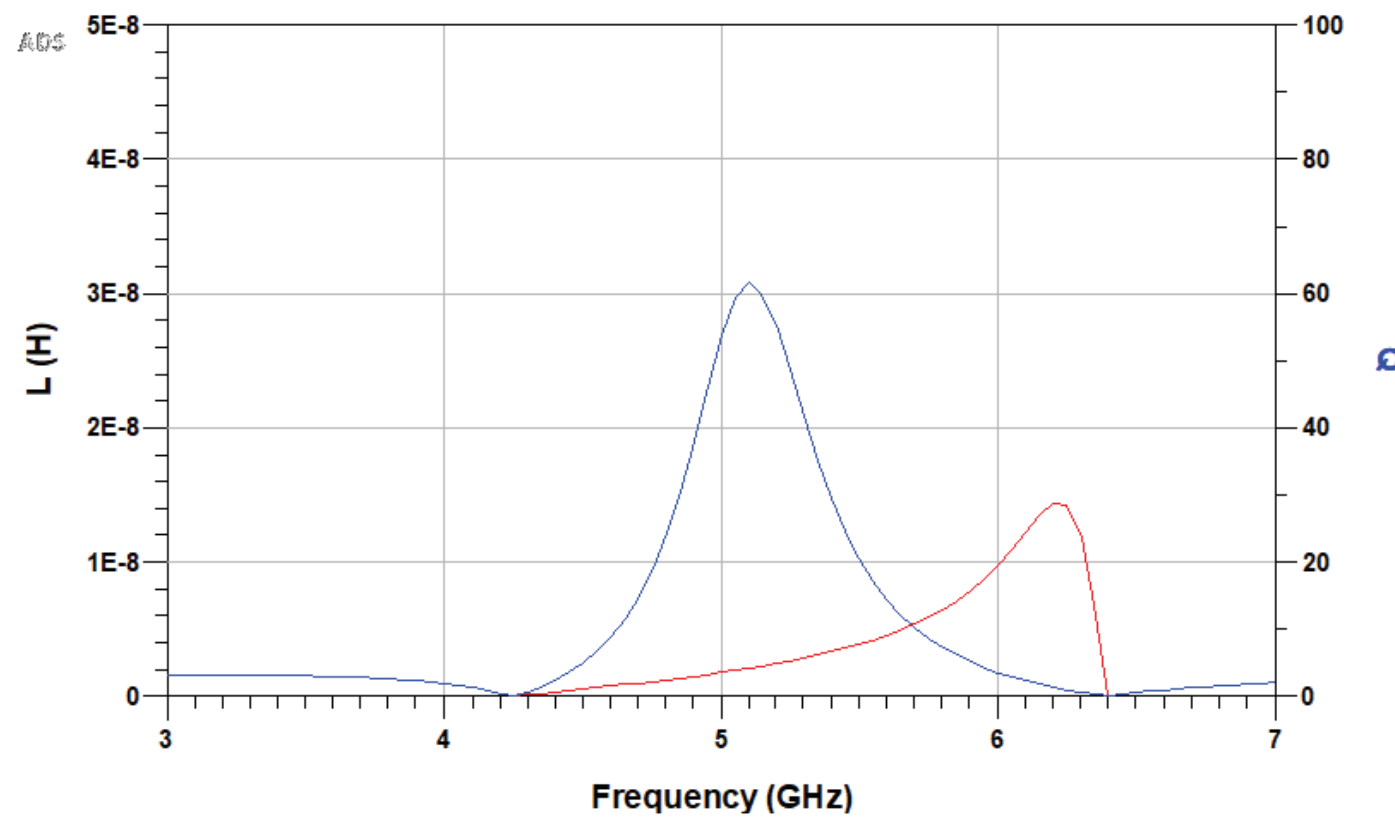

FiguRE 3.10: Schematic with real biasing simulation results

With the topology finalized the schematic design was laid-out in ADS Momentum. The circuit was laid-out on a $2 \mathrm{~mm}$ X $2 \mathrm{~mm}$ die although it only took a portion of the area since space was needed for the band pass filter.

Since the active inductor is a feedback circuit, the main concern in layout was to minimize to signal time of flight around the loop and minimize the phase error. For this reason, the design was not laid-out using typical RF / microwave layout techniques such as controlled impedances. Rather the interconnect was kept as short as possible in an attempt to reduce the loop phase error and avoid any transmission line effects.

A ground fill was not applied to either of the metal interconnect layers as this had the effect of introducing stray capacitance all around the circuit resulting in poor performance.

Landing pads for probes were selected to be $100 \mathrm{um}$ wide at a pitch of $150 \mathrm{um}$. 


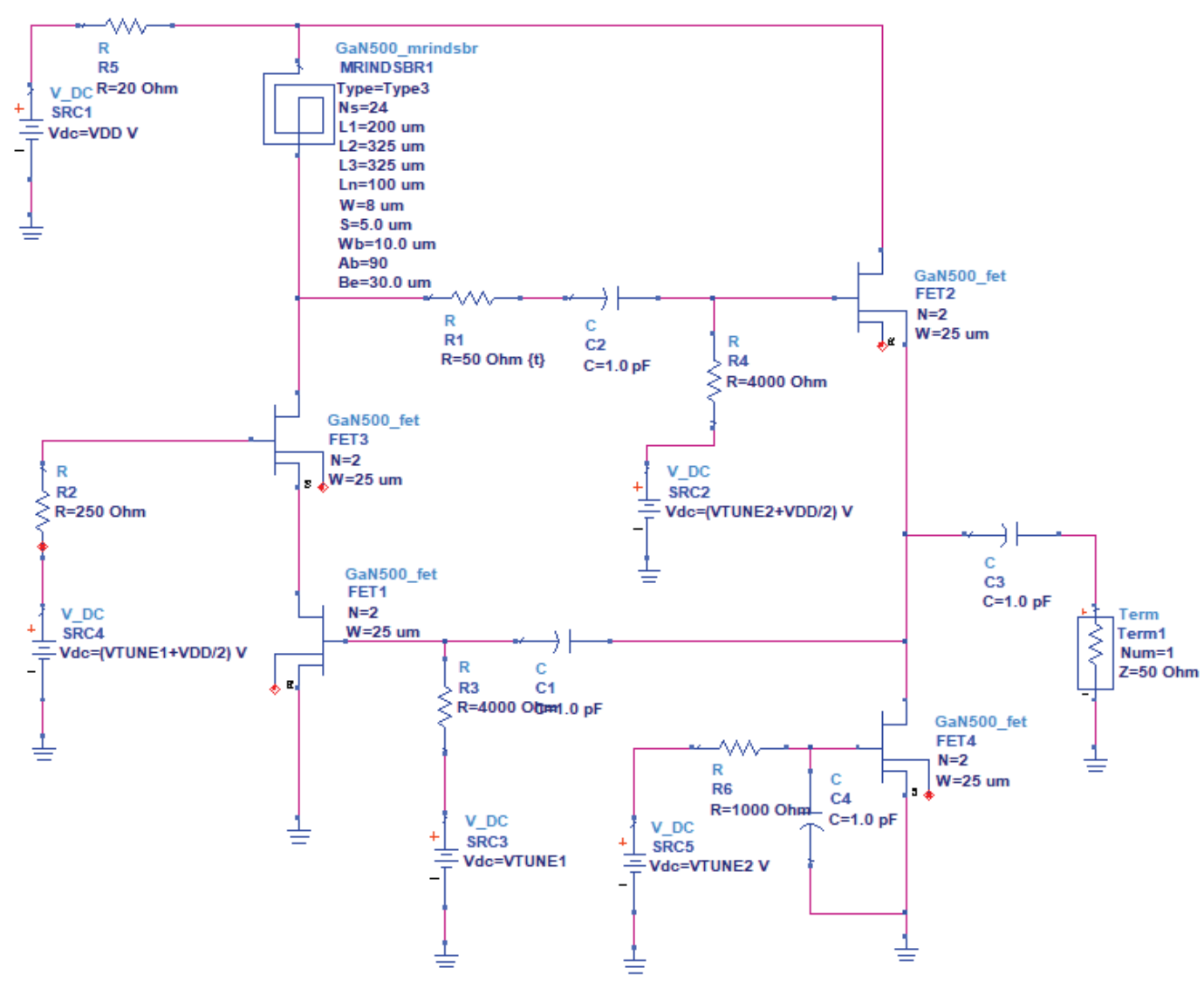

Figure 3.11: Final Schematic Topology, active biasing introduced

After several iterations of tweaking component values, optimizing component placement, and optimizing routing / interconnect, the layout was finalized in accordance with all design rules of the GaN500 v3.0 design kit.

The layout of the entire $2 \mathrm{~mm} X 2 \mathrm{~mm}$ die is shown in Figure 3.13. An enlargement of the active inductor region of the chip is shown in Figure 3.14.

With the layout finalized, a schematic-EM co-simulation was performed. The simulated inductance and $\mathrm{Q}$ factor are plotted in Figure 3.15. It can be seen that the active inductor is inductive from approximately $3.5 \mathrm{GHZ}$ to $6.5 \mathrm{GHz}$. The frequency 


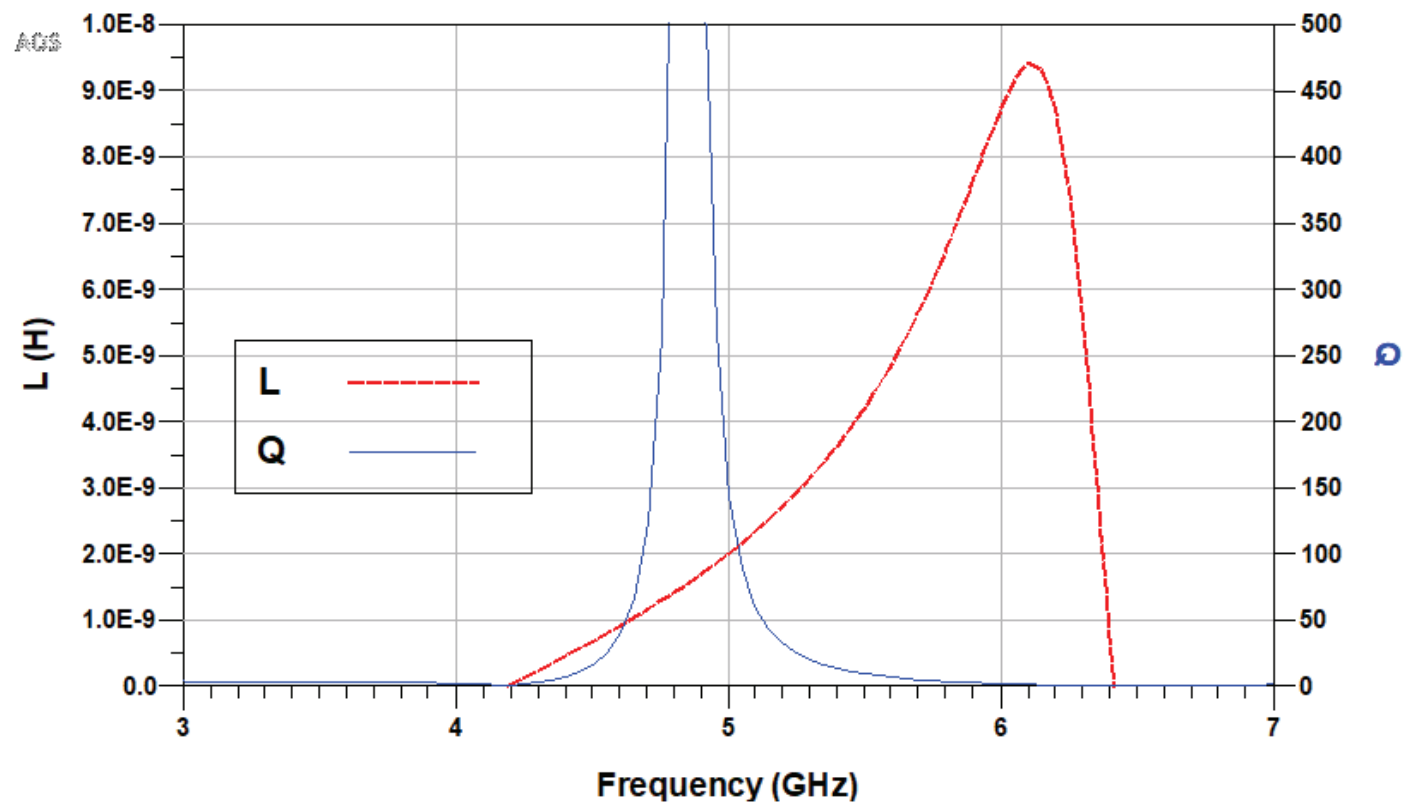

Figure 3.12: Final Schematic Simulation Results

range over which the inductor has a Q-factor greater than 10 is $3.9 \mathrm{GHz}$ to $5.4 \mathrm{GHz}$. As is evident the inductor has a large dependence upon frequency.

Figure 3.16 shows the inductance and Q-factor at $5 \mathrm{GHz}$ as a function of tuning voltage VTUNE1 for several values of VTUNE2. The active inductor has a useful tuning range of roughly $2.5 \mathrm{nH}$ to $7 \mathrm{nH}$ over a VTUNE1 tuning range of $-3.5 \mathrm{~V}$ to $1.5 \mathrm{~V}$. The tuning voltage VTUNE2 can simultaneously be used to tune the Q-factor while having a small effect on the inductance. VTUNE1 can therefore be used to set the desired inductance whilst VTUNE2 can be adjusted so that the quality factor is optimized at that particular inductance.

Figure 3.17 shows the inductance and quality factor versus VTUNE2 at 5GHz for several values of VTUNE1. This reiterates how the inductance can be tuned using VTUNE1 and then VTUNE2 can be used to adjust the Q factor. 


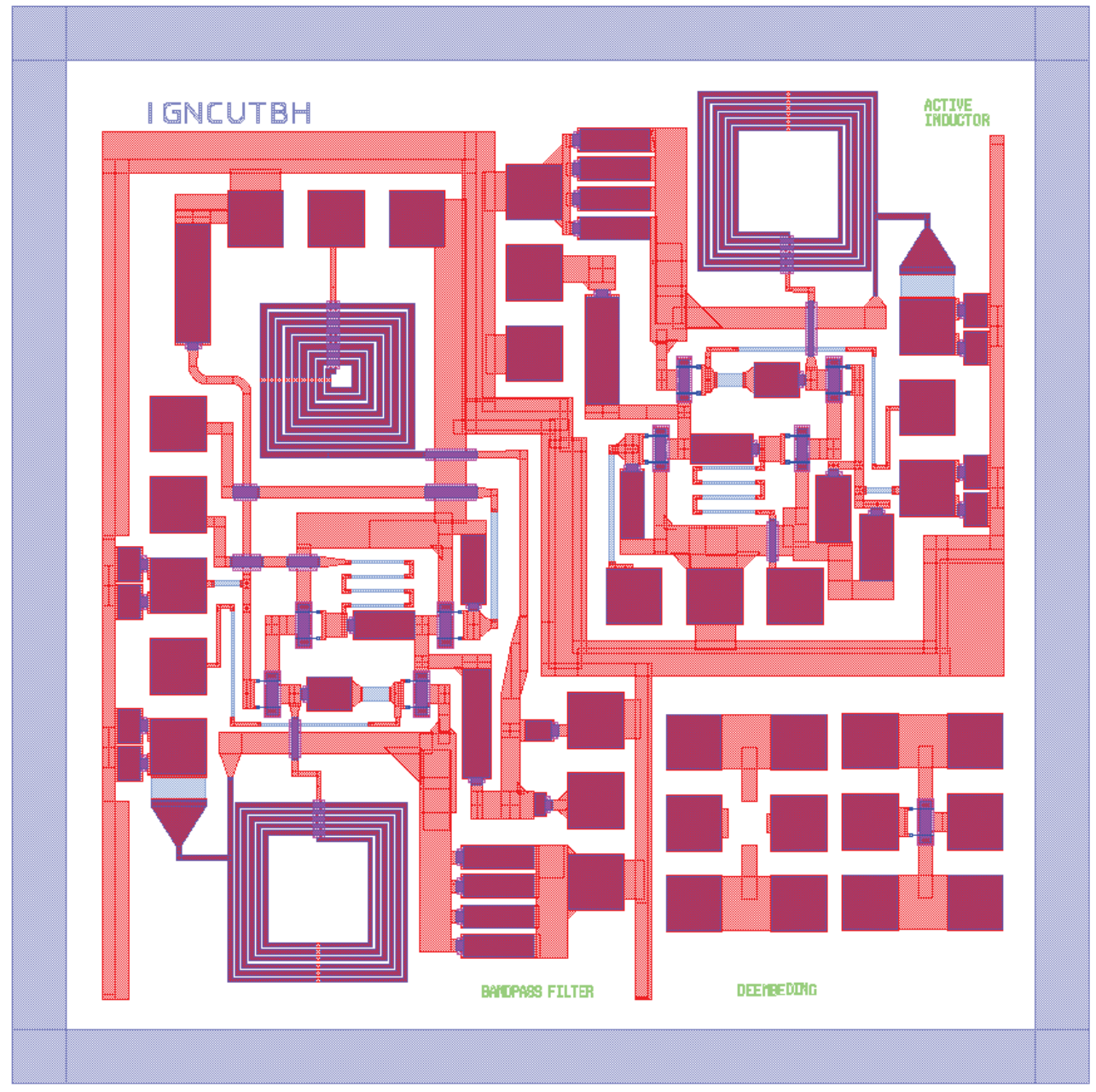

Figure 3.13: 2mm X 2mm Circuit Layout

\subsection{Bandpass Filter Design}

The simulation results from the active inductor demonstrated an inductive device with high tunable Q-factor but with a highly frequency dependent inductance. This frequency dependent behaviour would make the device unsuitable for operation in an application such as a voltage controlled oscillator. However, the frequency dependent behaviour can be exploited for the purpose of a band pass filter. The frequency 


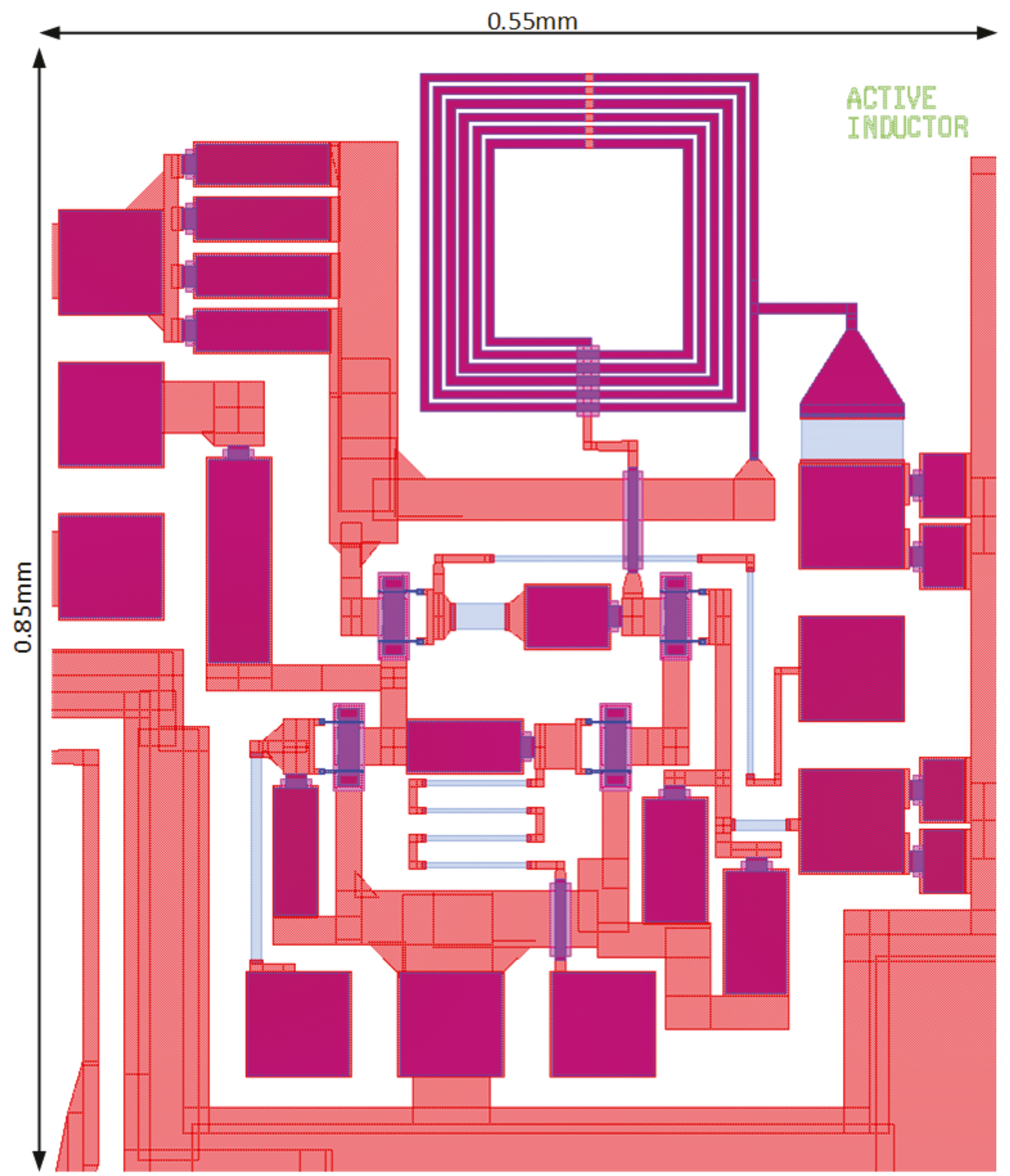

Figure 3.14: Active Inductor Layout

increasing inductance effectively results in a high order roll-off when incorporated in a band-pass or low-pass filter.

The active inductor was incorporated in a band-pass filter using the basic topology shown in Figure 3.18. The active inductor resonates with $\mathrm{C} 2$ to form a bandpass 


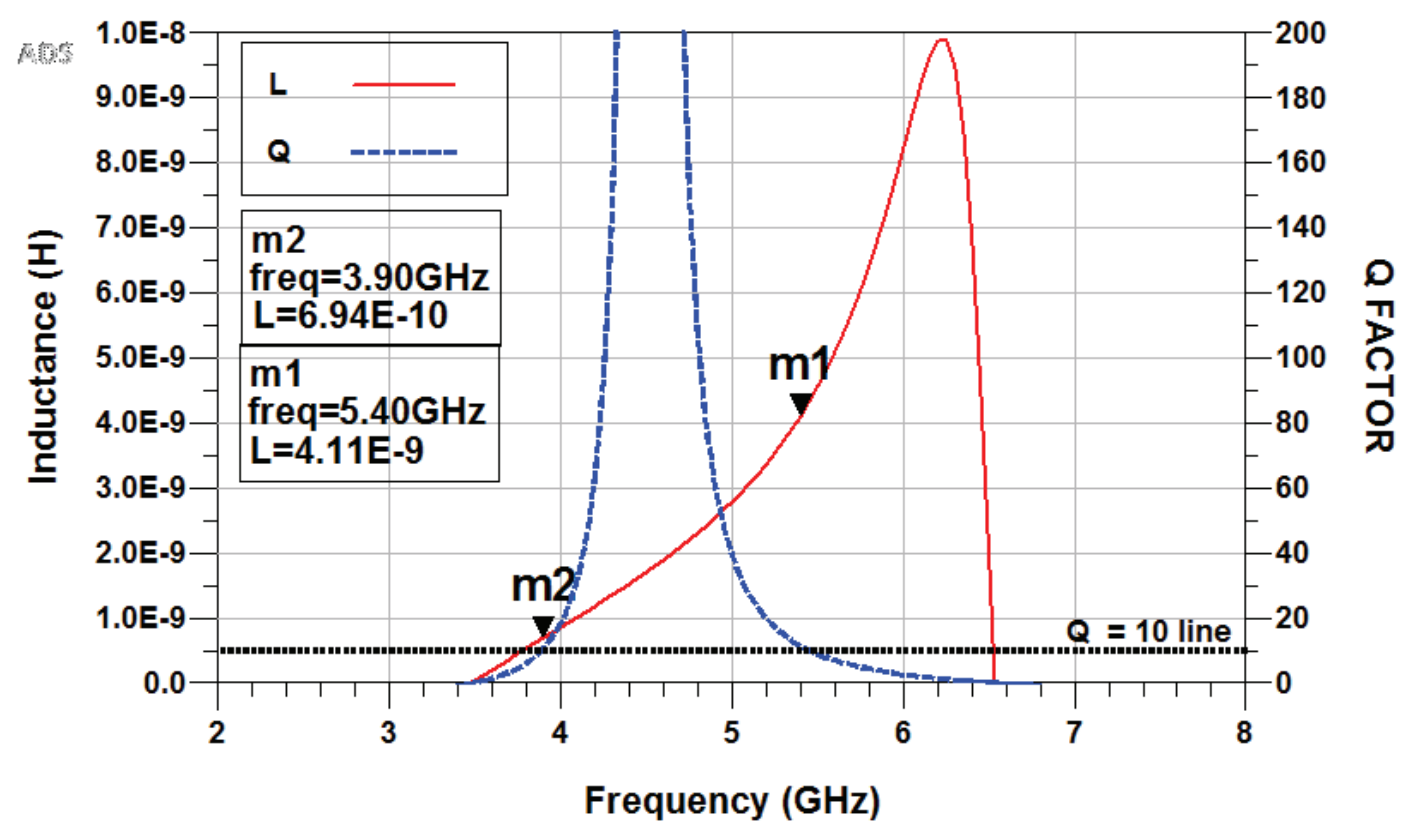

Figure 3.15: Final Layout Co-Simulation; Inductance and Q vs Frequency

around $5 \mathrm{GHz}$. $\mathrm{C} 1$ provides additional filtering at low frequencies whereas the fixed output inductor L1 provides additional filtering at high frequencies.

The bandpass filter was laid-out alongside the stand-alone active inductor on the same $2 \mathrm{mmX} 2 \mathrm{~mm}$ die. The complete layout was previously illustrated in Figure 3.13. An enlargement of the band-pass filter layout section alone is shown in Figure 3.19

With the BPF layout complete, a final EM-schematic co-simulation was performed. The results are shown in Figure 3.20

The filter demonstrates a nominal center frequency of $5 \mathrm{GHz}$ and a frequency tuning range of approximately $500 \mathrm{MHz}$ or $10 \%$ while maintaining a gain of $5 \mathrm{~dB}$. The -30 dB S21 on the high side is limited by the self-resonance of the fixed inductor. The increase in $\mathrm{S} 21$ below $3.5 \mathrm{GHz}$ is caused by the active inductor becoming capacitive at this frequency as is evident in Figure 3.15. 


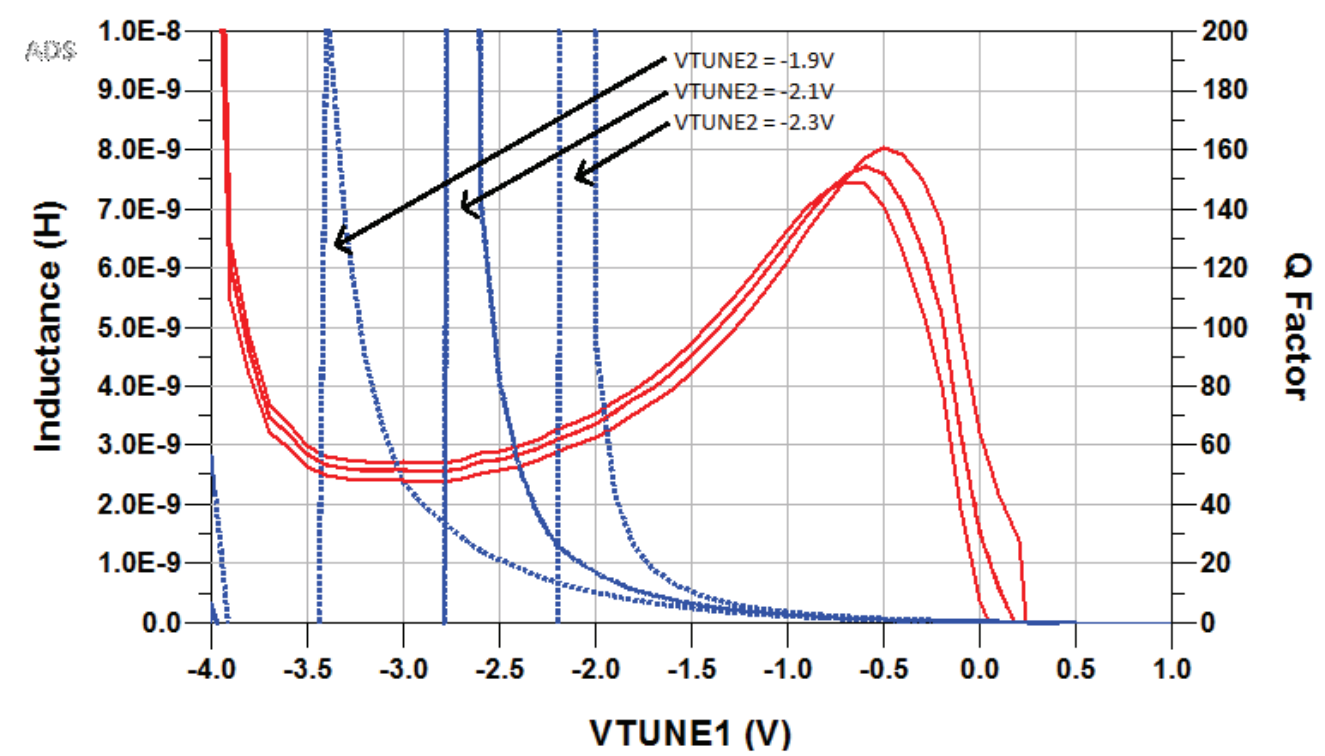

FiguRE 3.16: Final Layout Co-Simulation; Inductance and Q vs VTUNE1

Figure 3.21 shows the simulated filter passband bandwidth when tuned to $5 \mathrm{GHz}$. The simulated $3 \mathrm{~dB}$ bandwidth is measured to be $25 \mathrm{MHz}$. The simulated 10dB passband is measured to be $77 \mathrm{MHz}$. Defining the Q-factor of the filter to be $Q=f_{c} / B W_{3 d B}$, where $f_{c}$ is the center frequency and $B W_{3 d B}$ is the $3 \mathrm{~dB}$ bandwidth, the Q-factor in this case works out to be 200 .

A harmonic balance simulation was performed to establish the device's linearity. The output $1 \mathrm{~dB}$ compression point was measured at several frequencies for programmed gains of $+5 \mathrm{~dB},+2 \mathrm{~dB}$ and $-3 \mathrm{~dB}$. The simulated results are shown in Figure 3.22. The device exhibits better linearity when tuned for lower gain (nominally $-9 \mathrm{dBm}$ at $-3 \mathrm{~dB}$ gain versus $-14 \mathrm{dBm}$ for $+2 \mathrm{dBm}$ gain and $-16 \mathrm{dBm}$ for $+5 \mathrm{~dB}$ gain). These values would make the device ideal for a front end filter in a receiver where the expected received power would be far less than the device's P1dB. 


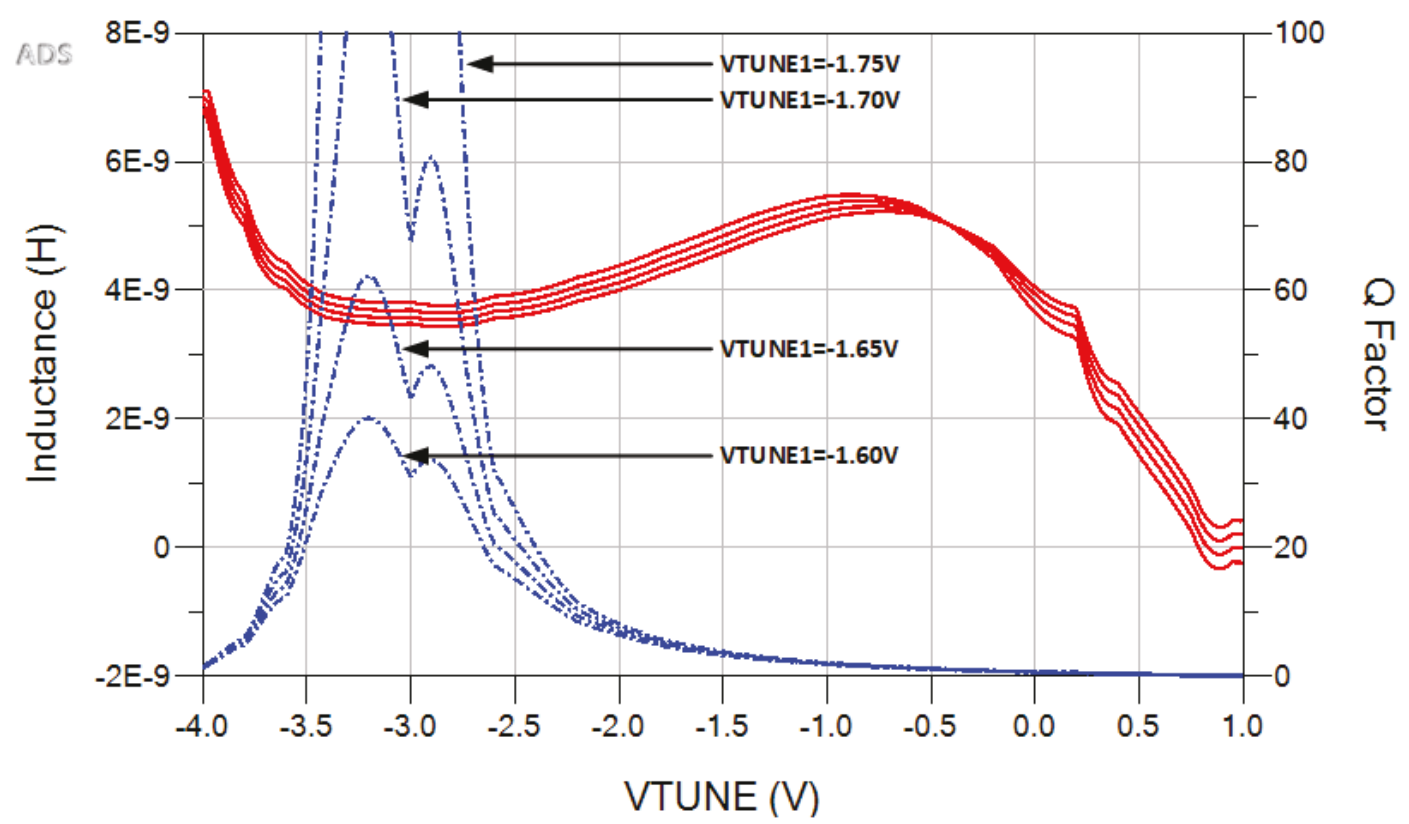

Figure 3.17: Final Layout Co-Simulation; Inductance and Q vs VTUNE2

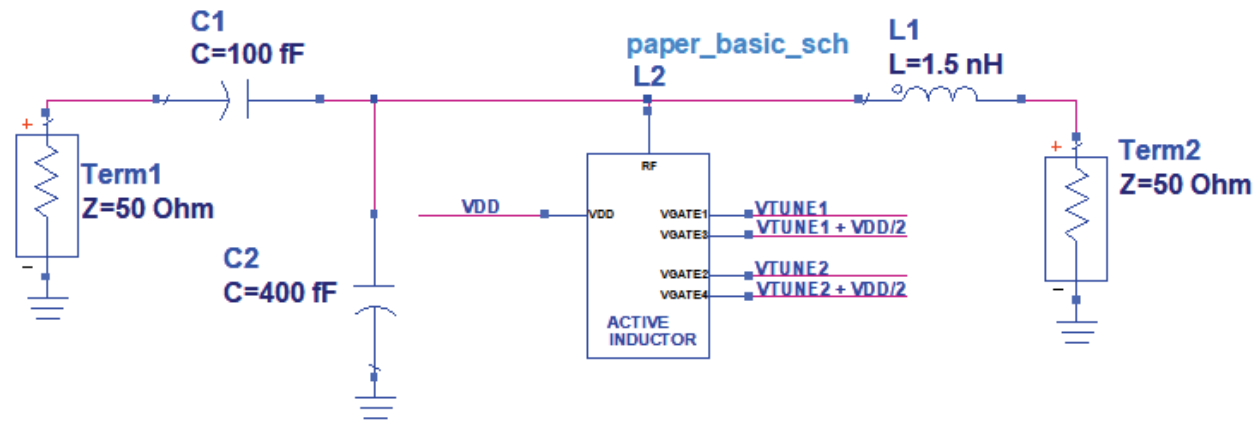

Figure 3.18: Bandpass Filter Topology

\subsection{Summary}

This chapter the presented the design and simulation of an active inductor in GaN along with a bandpass filter incorporating the GaN active inductor.

Firstly, a presentation of the NRC GaN500 was provided. The layer structure of the HEMTs was described along with a description of all passive components available 


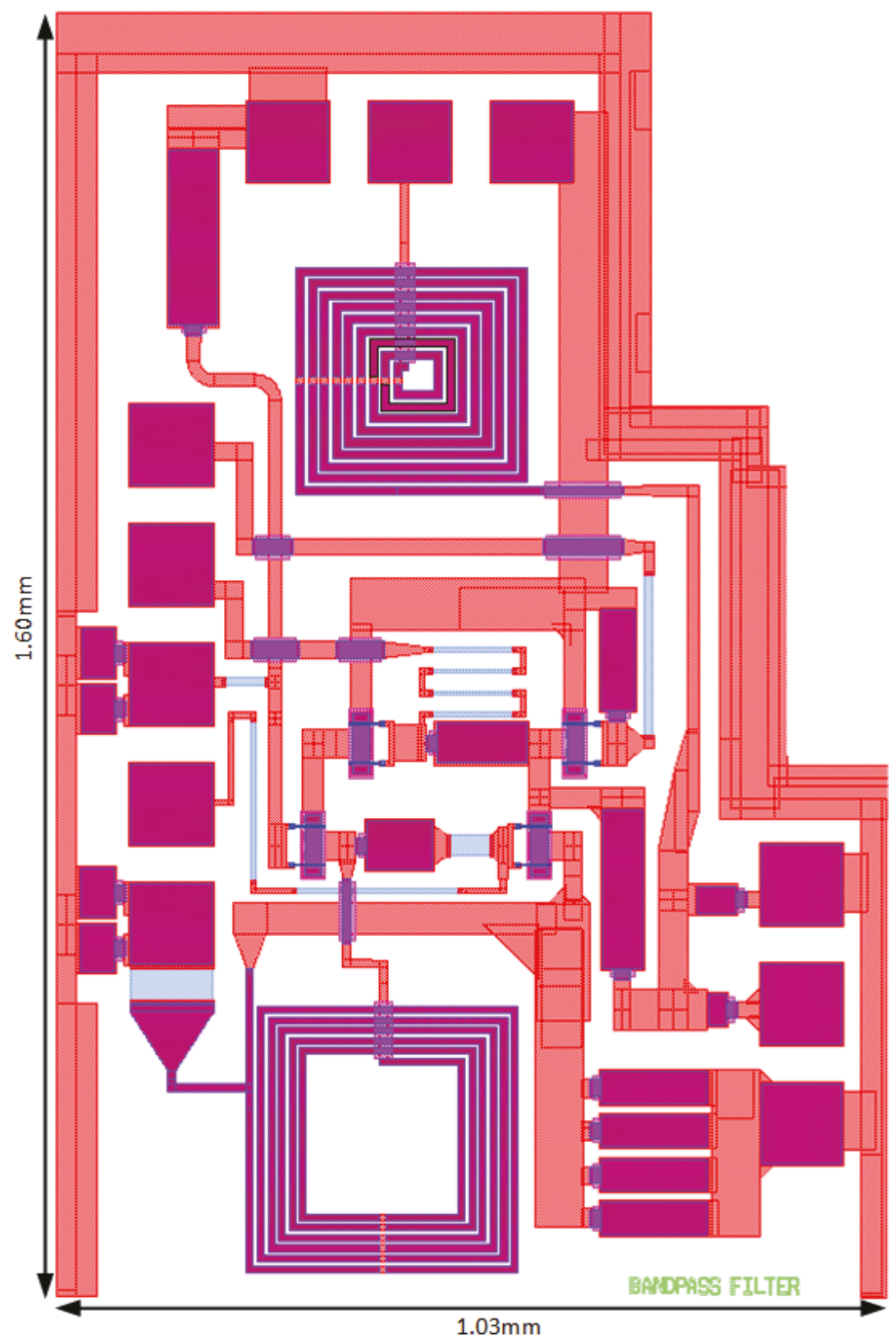

Figure 3.19: Bandpass Filter Layout

within the design kit.

The design goals of the active inductor were then presented establishing a nominal operating frequency of $5 \mathrm{GHz}$ with a nominal inductance of $1 \mathrm{nH}$ targeted. Design 


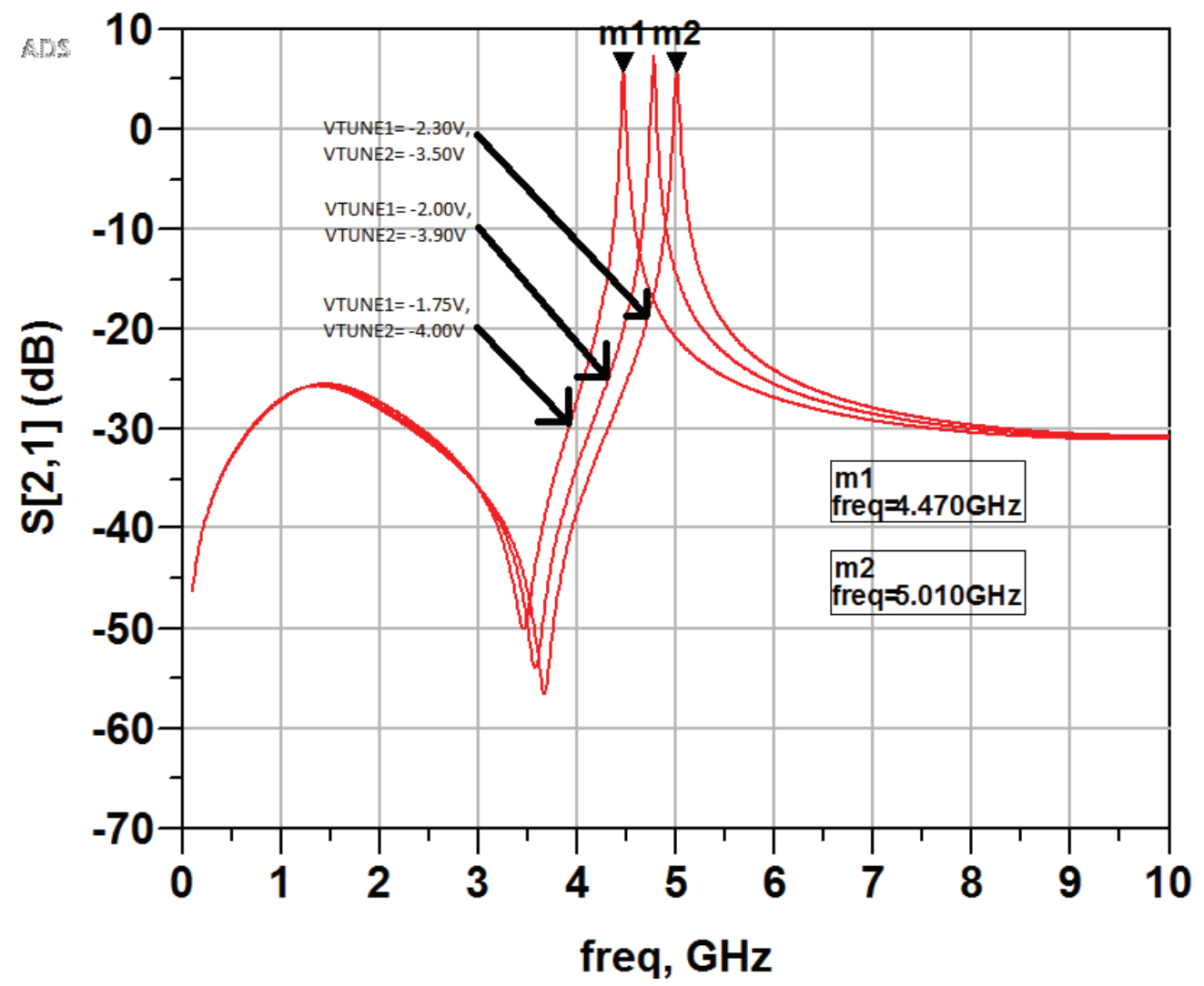

Figure 3.20: Bandpass Filter Simulated Tuning Range

considerations, taking into account the limitations of the design kit among others, were then presented.

Once the design goals and design considerations / limitations were established two active inductor topologies were presented with the advantages and disadvantages of each listed. The common source - common drain topology was chosen due to it having an extra degree of freedom in tuning.

The design of the active inductor was then presented showing schematics and simulation results of each design iteration from initial idealized circuit to final EM-schematic cosimulation. Simulations were given showing inductance and quality factor versus frequency, and versus tuning voltages VTUNE1 and VTUNE2. The device was shown 


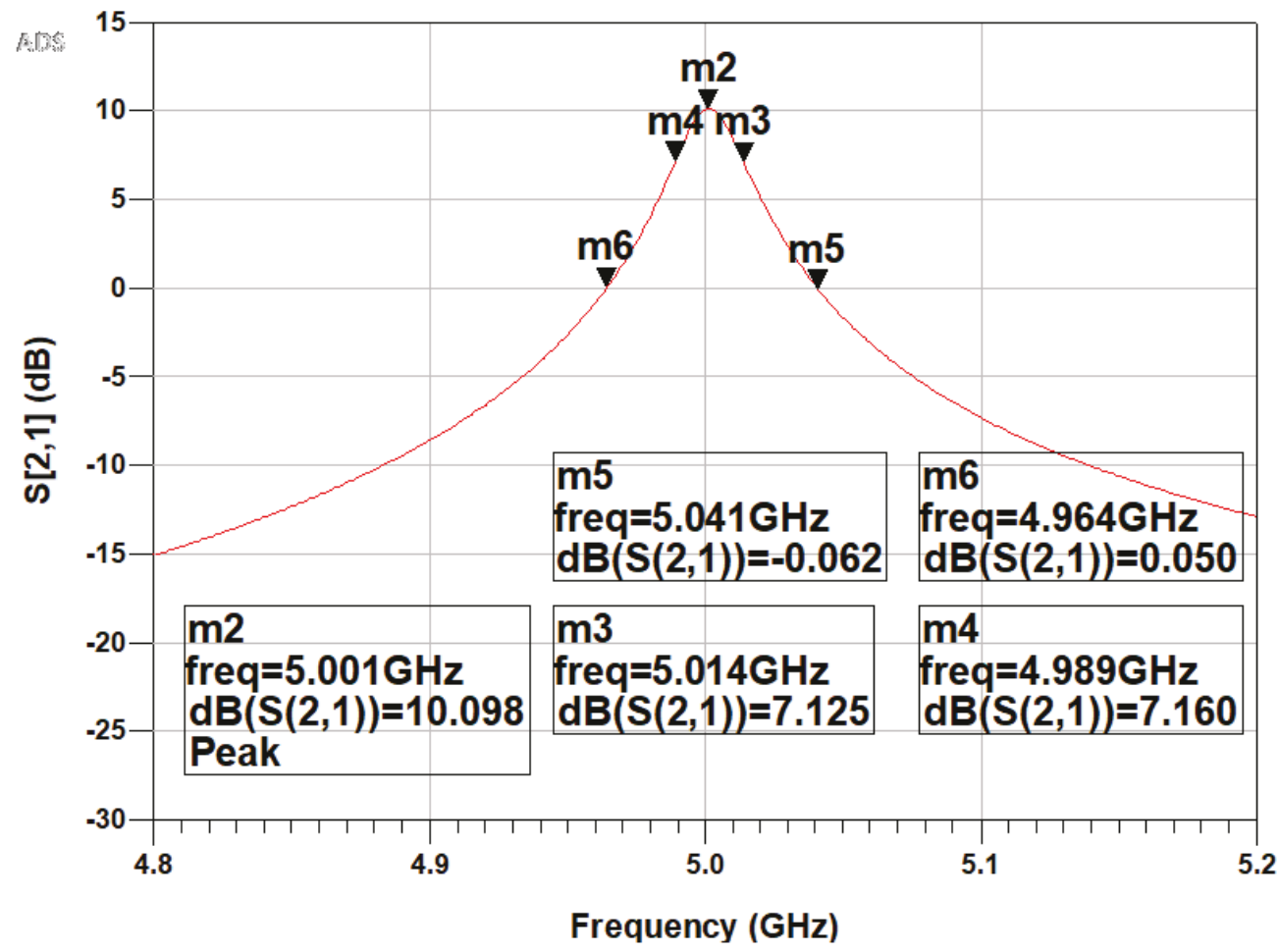

Figure 3.21: Bandpass Filter Simulated Pass Band

to be inductive over a frequency range of $3.5 \mathrm{GHz}$ to $6.5 \mathrm{GHz}$ but demonstrated a strong resonance at higher frequencies. The simulations showed Q values of several hundred depending on the tuning settings.

Finally, the bandpass filter design incorporating the active inductor was presented. Simulations showed a tunable frequency range of $4.5 \mathrm{GHz}$ to $5 \mathrm{GHz}$ while maintaining a gain of $5 \mathrm{~dB}$. The $3 \mathrm{~dB}$ and $5 \mathrm{~dB}$ passbands were measured to be $25 \mathrm{MHz}$ and $77 \mathrm{MHz}$ respectively.

The final layout to be fabricated was presented. The measured results from the fabricated device are present in Chapter 4. 


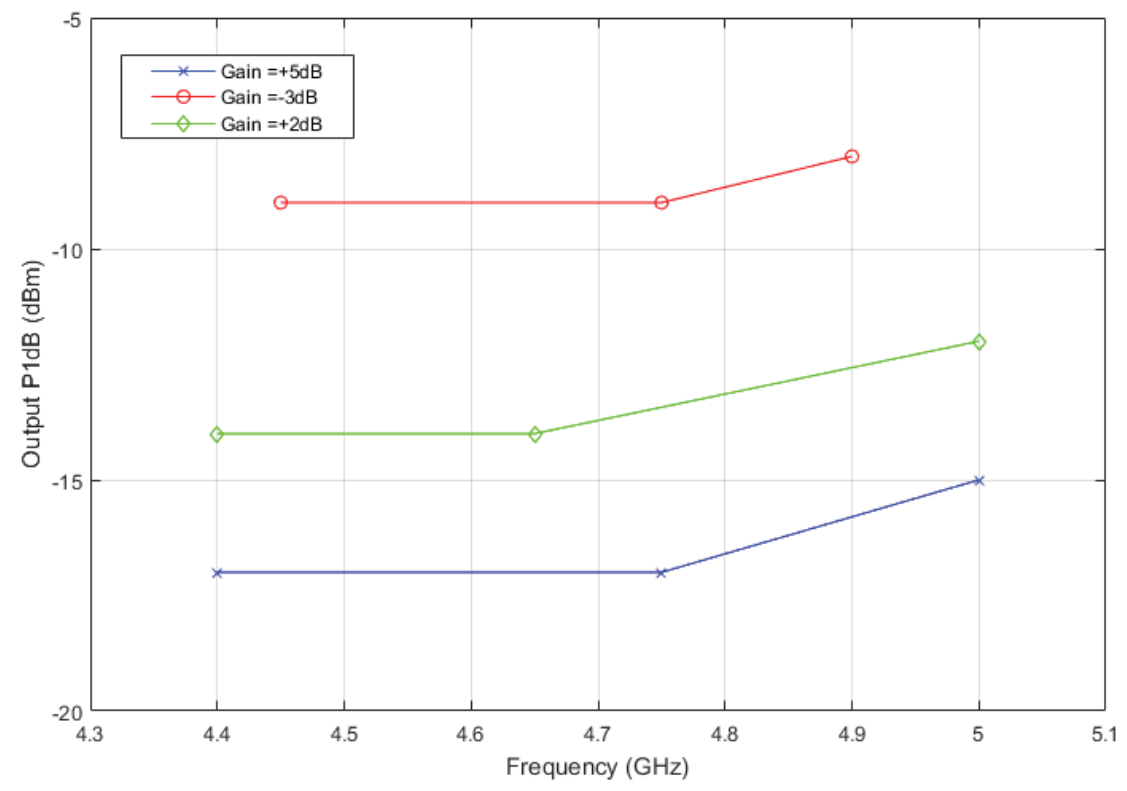

Figure 3.22: Bandpass Filter Simulated Linearity 


\section{Chapter 4}

\section{Results}

The IC was sent for fabrication at the NRC GaN foundry via the CRC. Figure 4.1 shows a view of the fabricated die under a microscope.

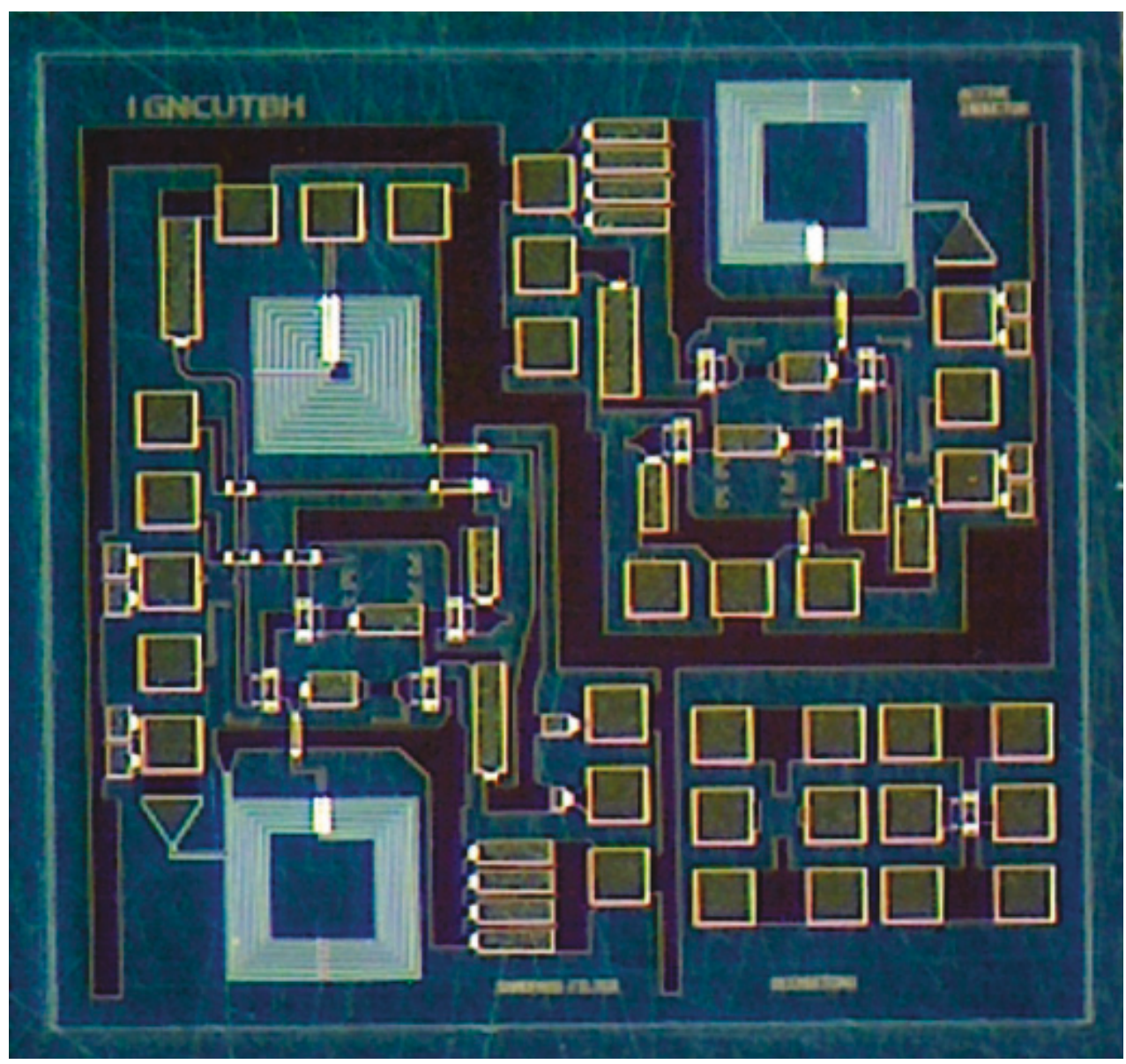

Figure 4.1: View of fabricated IC under microscope (2mm x 2mm) 
The active inductor can be observed in the top right corner, whereas the bandpass filter incorporating the active inductor occupies the left side of the die. At the bottom right is a single FET in common source configuration for characterization.

\subsection{General Equipment Setup}

The basic test equipment setup is shown in Figure 4.2.

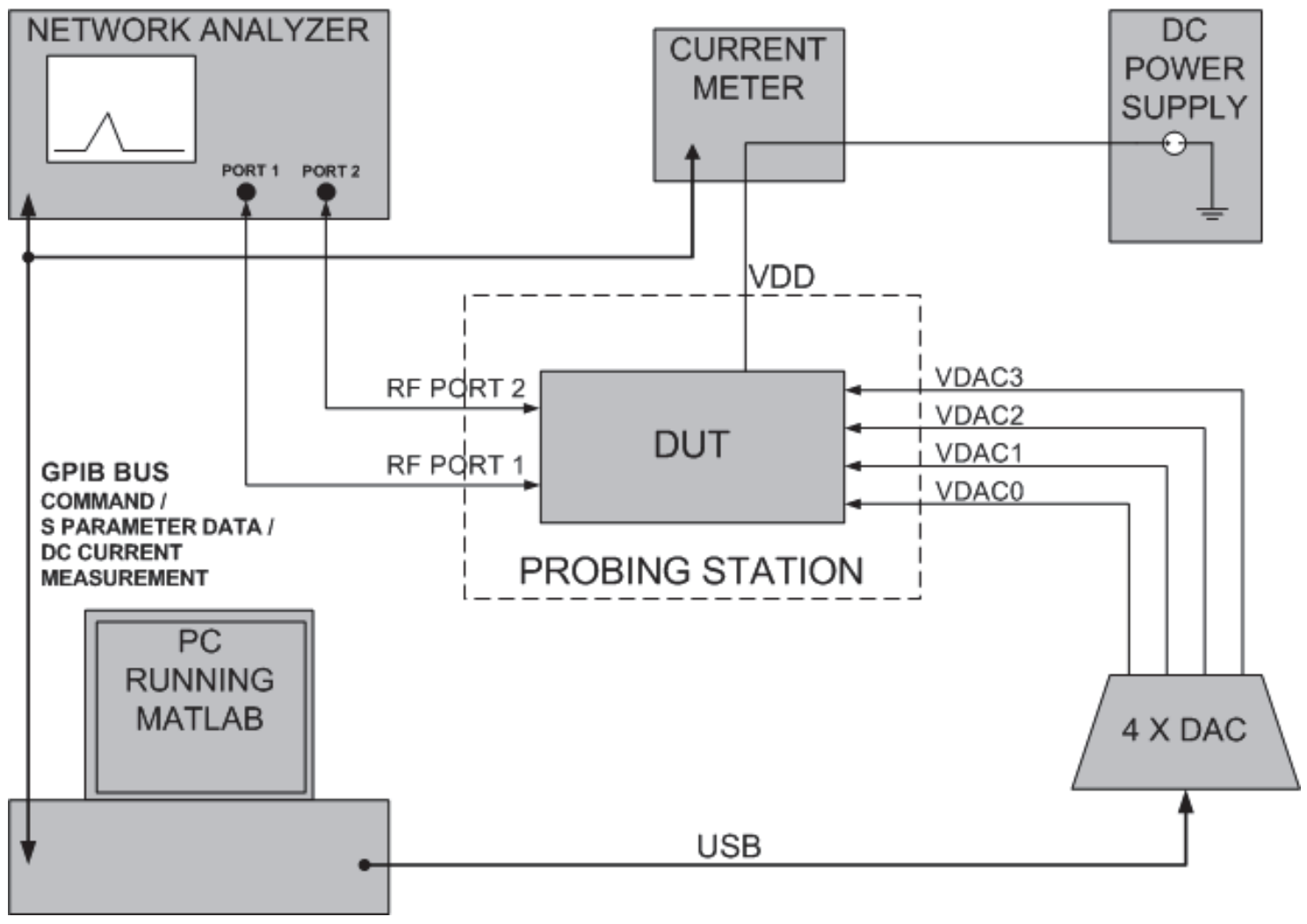

FiguRe 4.2: Block diagram of ideal gyrator

All measurements whether for device characterization, active inductor characterization, or filter characterization were performed using some slight variation of this setup. 
Table 4.1 lists the major test components used during testing

TABLE 4.1: List of test equipment

\begin{tabular}{|l|l|}
\hline Part Number & Description \\
\hline Agilent 8722ES & $50 \mathrm{MHz}-40 \mathrm{GHz}$ Vector Network Analyzer \\
\hline Agilent 82357B & USB-GPIB Adaptor \\
\hline Agilent 34401A & Digital Multimeter \\
\hline N/A & MicroZoom Micoscope / Probing Station \\
\hline Measurement Computing USB3101 & 4 channel 16 bit USB controlled DAC \\
\hline Picoprobe & 2X 150 um GSG probe \\
\hline Picoprobe & 150 um pitch DC probes (various) \\
\hline
\end{tabular}

The USB DAC supplies 4 analog outputs for controlling gate bias voltages.

For single port measurements, only port one of the VNA is used to measure $S_{11}$. For the filter and device characterization, both VNA ports were used.

The Agilent Digital Multimeter was used to measure current on the VDD supply.

The VNA and Digital Multimeter were connected to a PC via GPIB. The 4 channel DAC was connected to the PC via USB.

The PC was running Matlab 2015b 32 bit with the Data Acquisition and Instrumentation Toolboxes installed. This allowed software control of the devices and automation of the testing procedure.

All Matlab code is included in Appendix B. This includes code for controlling the test setup (gathering data and controlling analog voltages) and code for post-processing data. It should be noted that this test setup is not specific to the measurements needed in this thesis but is a generic test setup that can be used for characterizing the frequency response of any device requiring some analog control. For this reason, the full code is provided should future students require such a test setup. 
The actual test setup is shown in Figure 4.3 and Figure 4.4. Figure 4.3 shows the complete setup including the laptop, VNA, probing station, USB DAC, and power supply. Figure 4.4 shows the IC on the probing station.

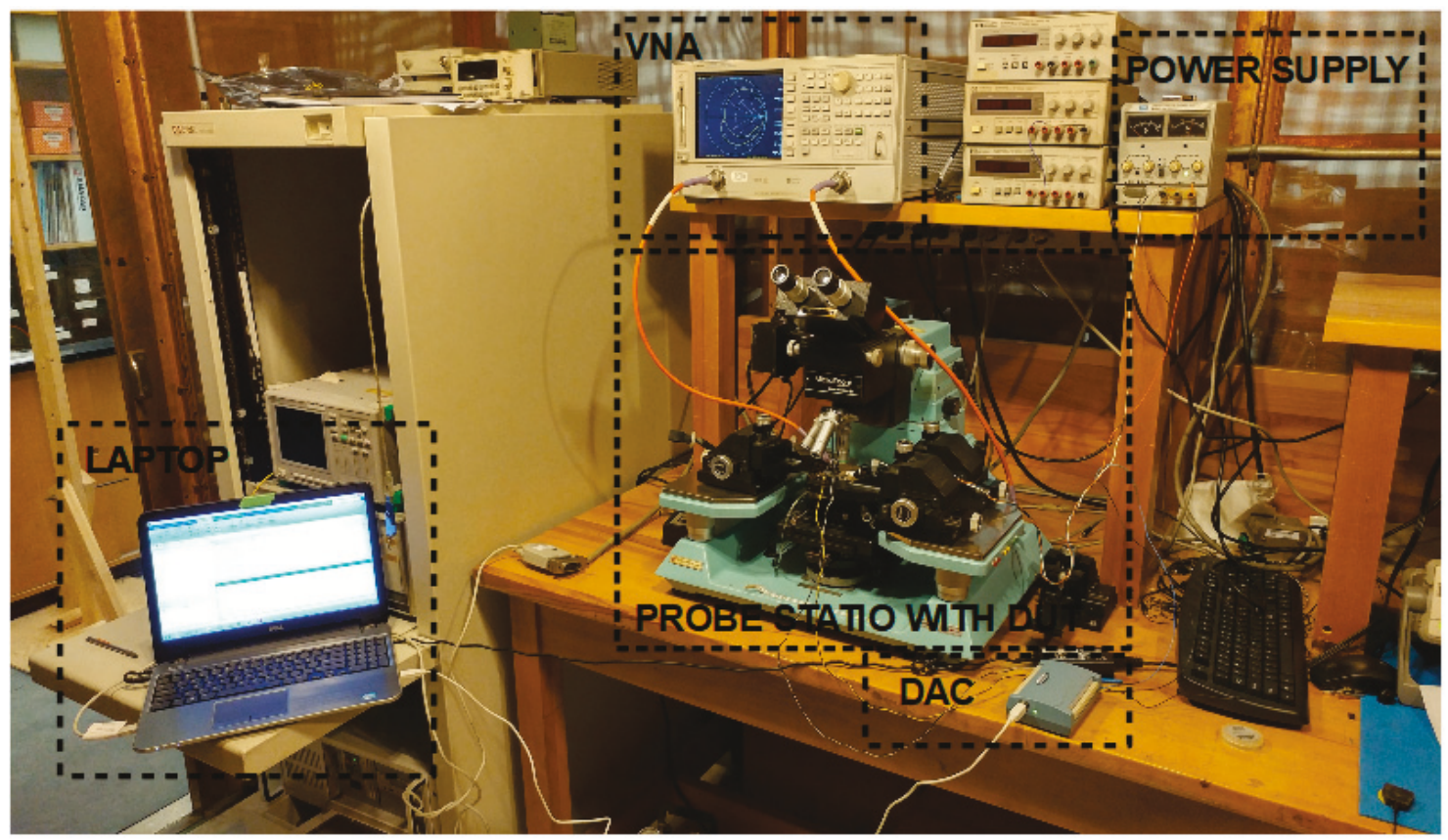

Figure 4.3: Photograph of Test Setup

Figure 4.5 shows a typical frequency sweep on the VNA of the active inductor.

\subsection{Device Characterization}

A single FET in common source configuration was included in the layout allowing characterization of the GaN devices. Ideally, transistors in common drain and common base configurations would have been included in the layout but space constraints did not permit this. 


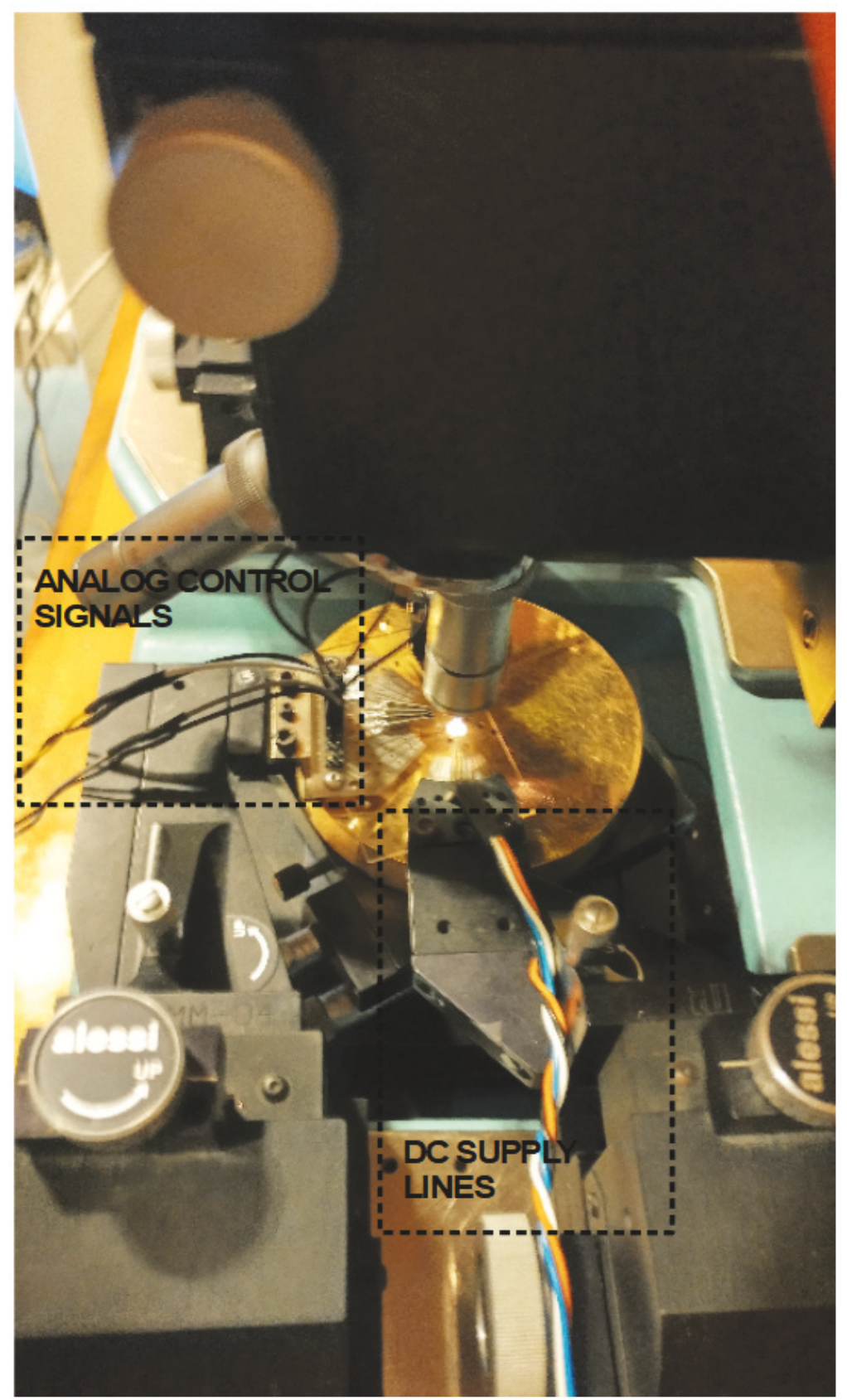

Figure 4.4: Photograph of IC on probing station

The GaN device was characterized for characteristic $I_{D}$ versus $V_{D S}$ behaviour and also for transit frequency $f_{t}$. 


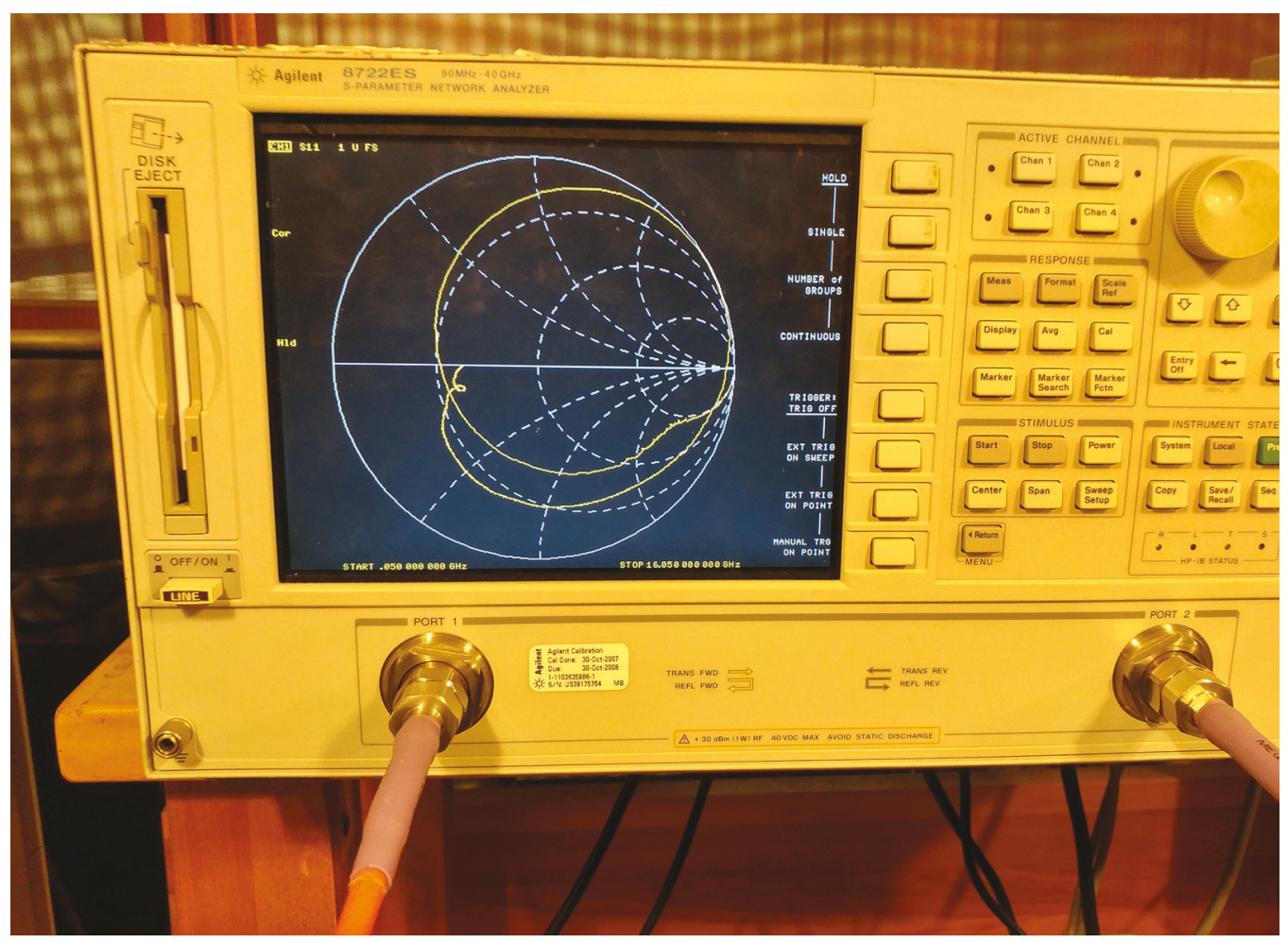

Figure 4.5: Typical VNA capture of active inductor

The measurements were repeated for two devices.

\subsection{1 $V_{D S}$ versus $I_{D S}$ Characterization}

Bias tees were connected to the RF probes on the gate and drain of the device. The DC coupled port of the gate bias tee was connected to a channel on the USB DAC allowing $V_{G S}$ to be swept by software. The DC coupled port of the drain bias tee was connected to the VDD power supply (with the Agilent multimeter in line). The AC coupled ports of the gate and drain bias tees were terminated for this measurement as only DC behaviour was of concern. 
The DAC was programmed to values of $-4,-3,-2,-1,0,1$ volts and at each point the current on the digital multimeter was read via GPIB and recorded in Matlab. This was repeated for $V_{D S}$ from $0 \mathrm{~V}$ to $20 \mathrm{~V}$. $V_{D S}$ was tuned manually.

The resulting curves for two devices are shown in Figure 4.6 and Figure 4.7 respectively.

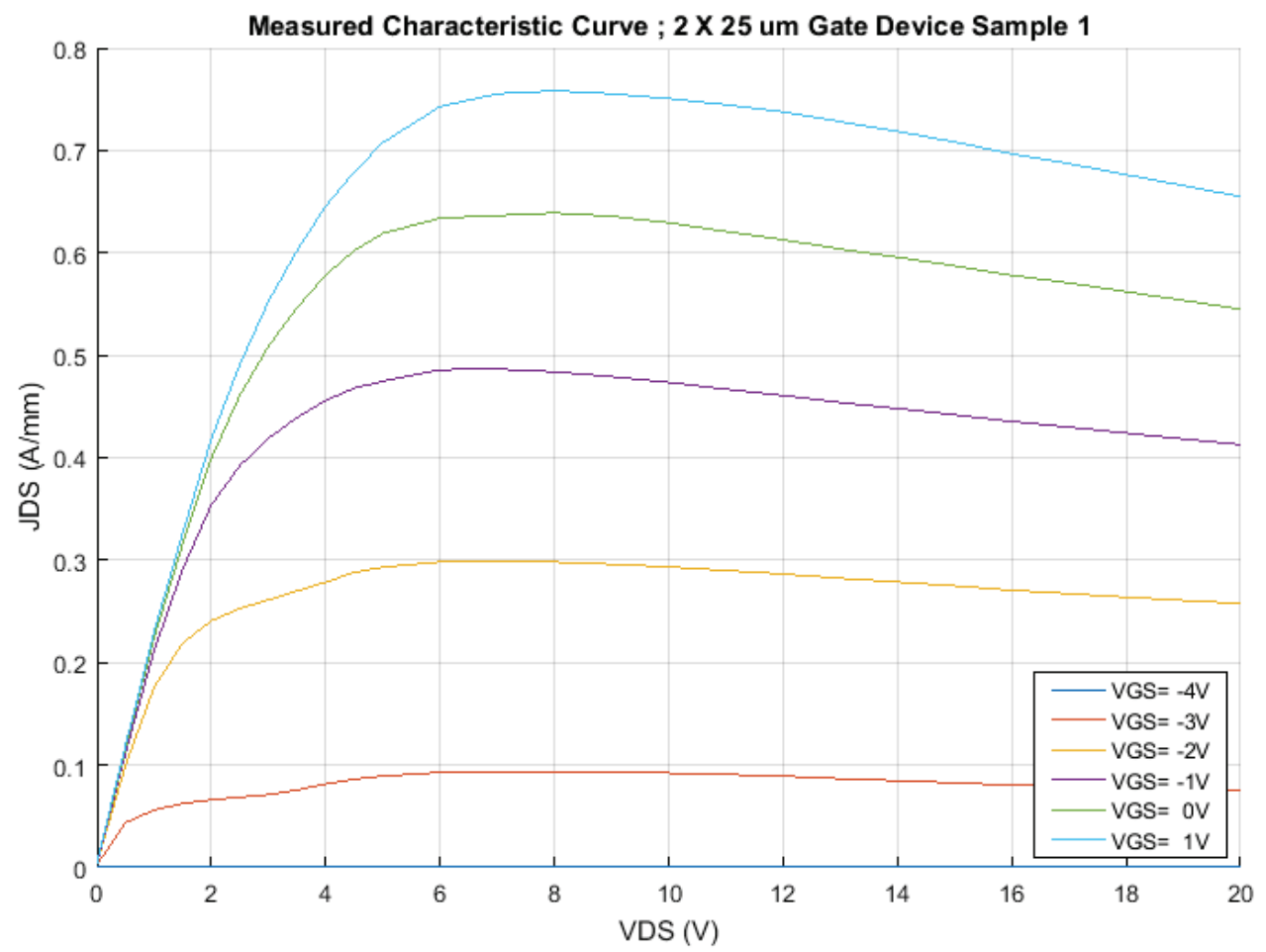

Figure 4.6: Characteristic curve, device 1

The characteristic curve from the GAN500 V3.01 manual is shown in Figure 4.8.

The current densities for $V_{D S}=10 \mathrm{~V}$ are tabulated for the 3 curves in Table 4.2.

It can be seen that at $V_{G S}=0 \mathrm{~V}$ there is a $14 \%$ variation in $J_{D S}$ between the two measured devices. Also, $J_{D S}$ is $16 \%$ higher for the published value than the average of the two measured values. 


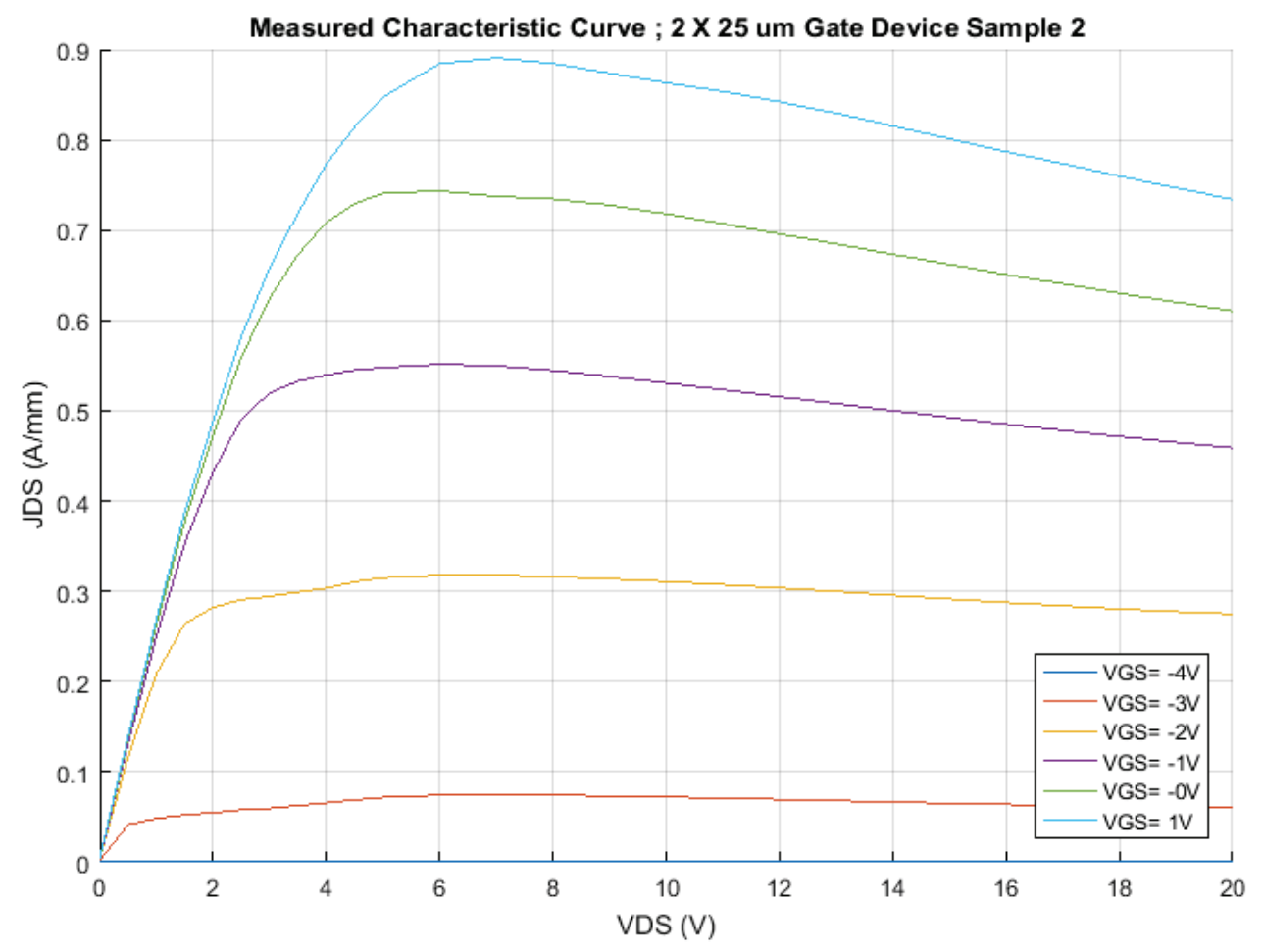

Figure 4.7: Characteristic curve, device 2

TABLE 4.2: $J_{D S}$ at $V_{D S}=10 \mathrm{~V}$

\begin{tabular}{|l|l|l|l|}
\hline VGS (V) & Device 1 & Device 2 & GaN500 Manual \\
\hline-4.0 & 0.0 & 0.0 & 0.02 \\
\hline-3.0 & 0.09 & 0.07 & 0.20 \\
\hline-2.0 & 0.29 & 0.31 & 0.44 \\
\hline-1.0 & 0.47 & 0.53 & 0.64 \\
\hline 0.0 & 0.63 & 0.72 & 0.81 \\
\hline
\end{tabular}

\subsection{2 $f_{t}$ Characterization}

Using the same setup as the previous section but with the gate bias tee's AC coupled port connected to port 1 of the VNA and the drain bias tee's AC coupled port connected to port 2 of the VNA, $f_{t}$ was characterized for two devices.

With VDS set to a particular voltage, a Matlab routine was run to sweep VGS from 


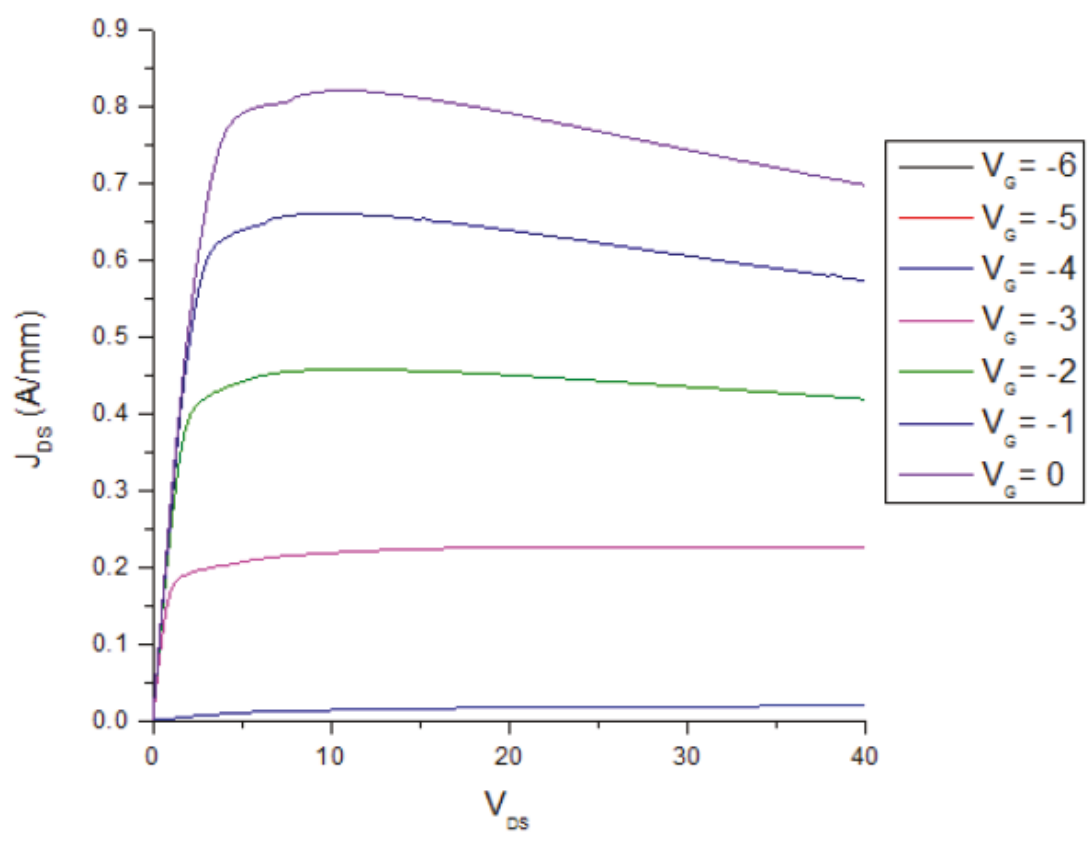

Figure 4.8: Characteristic curve, GaN500 V3.01 manual

$-4.0 \mathrm{~V}$ to $1.0 \mathrm{~V}$ in increments of $0.1 \mathrm{~V}$. At each point, the full 2 port S-parameters were read as was the current IDS from the Agilent multimeter. From the S-parameters, H-parameters could be derived and $f_{t}$ was found as the point at which the magnitude of $\mathrm{h} 21=0 \mathrm{~dB}$.

Plots of h21 for varying VGS at VDS $=10 \mathrm{~V}$ for device 1 are shown in Figure 4.9 to Figure 4.12

The $f_{t}$ for each $V_{G S}$ value was measured and a plot of $f_{t}$ versus $I_{D S}$ was obtained as shown in 4.13 .

The above procedure was repeated for $V_{D S}$ values of $5 \mathrm{~V}, 9 \mathrm{~V}, 9.5 \mathrm{~V}, 10.5 \mathrm{~V}, 11 \mathrm{~V}, 15 \mathrm{~V}$ and $20 \mathrm{~V}$ and the results are presented in Appendix A.1.

The complete procedure was repeated for the second device. The resulting $f_{t}$ versus $I_{D S}$ curves are plotted in Appendix A.2 for VDS varying from $5 \mathrm{~V}$ to $20 \mathrm{~V}$. 


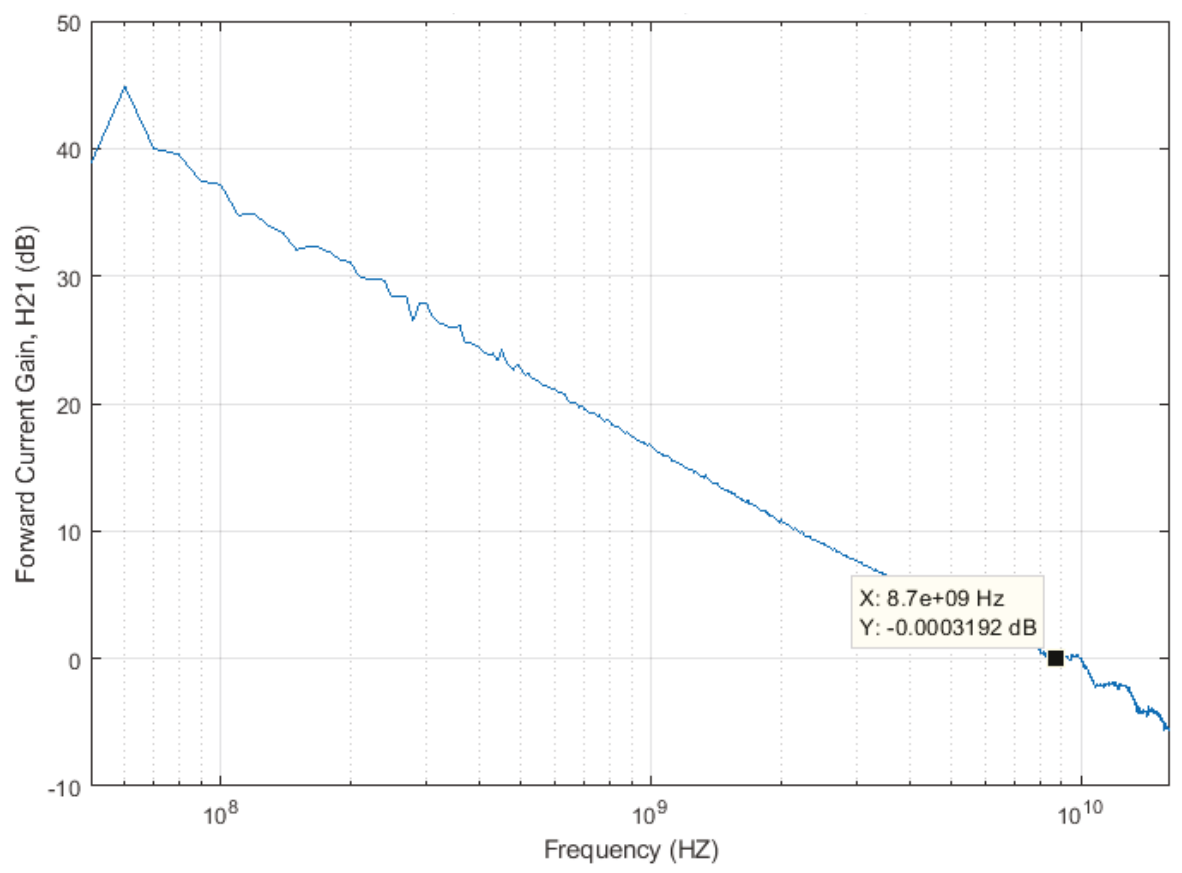

Figure 4.9: Ft vs h21, device 1 , VDS $=10 \mathrm{~V}, \mathrm{VGS}=-3 \mathrm{~V}$

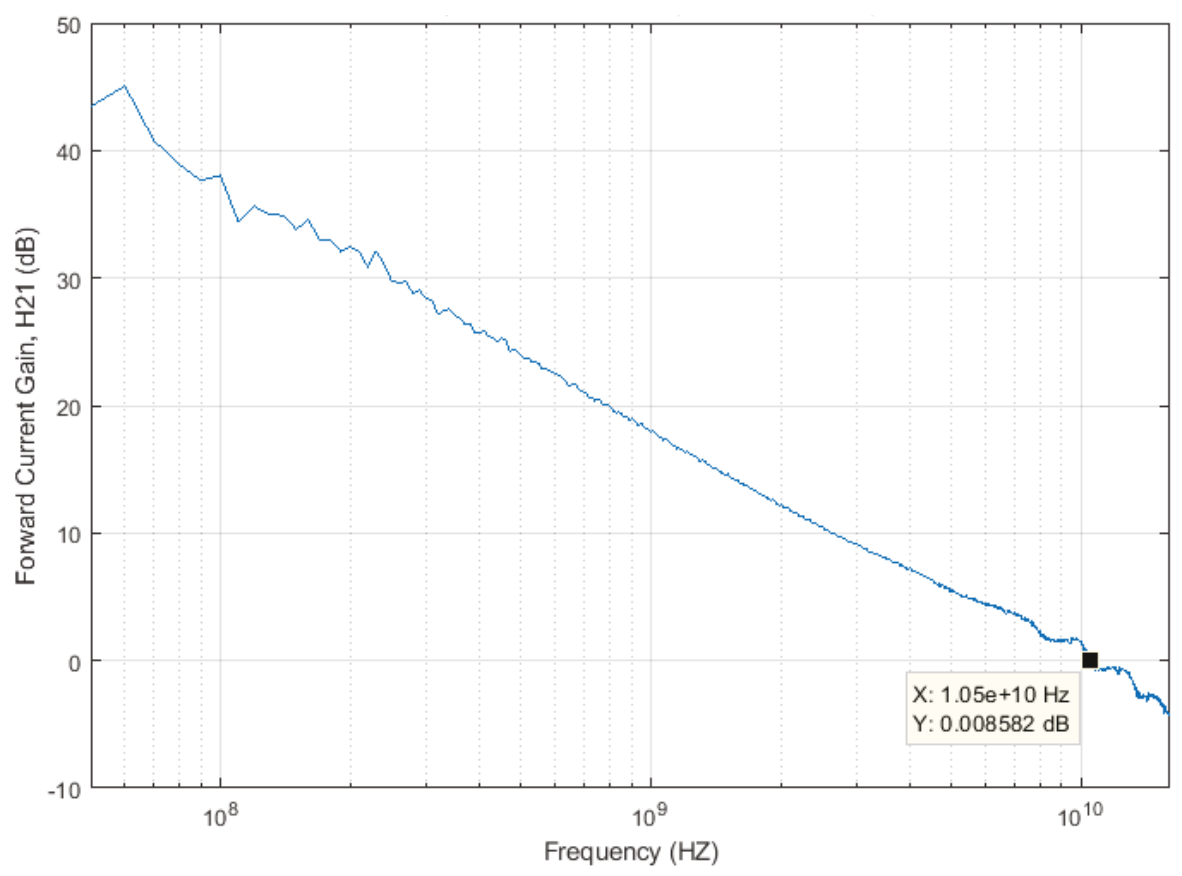

Figure 4.10: Ft vs h21, device $1, \mathrm{VDS}=10 \mathrm{~V}, \mathrm{VGS}=-2 \mathrm{~V}$ 


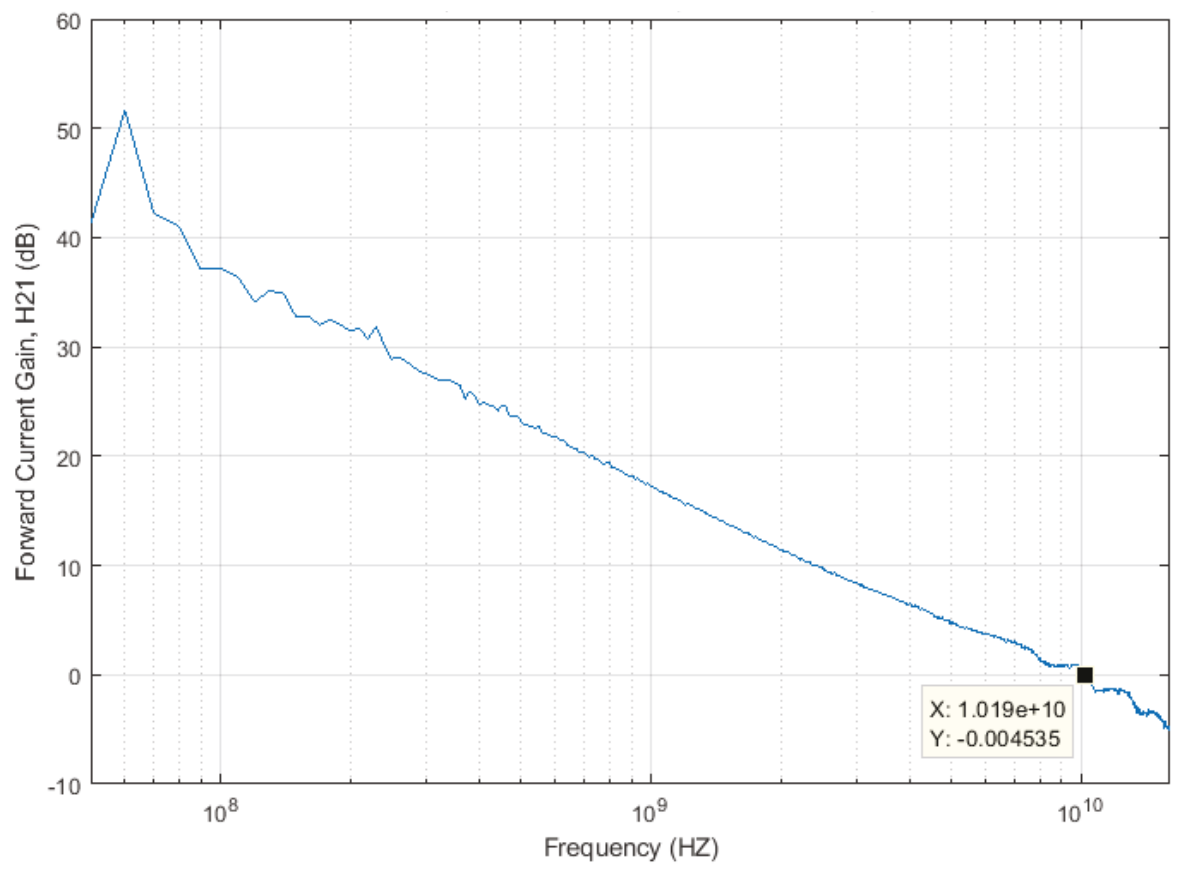

Figure 4.11: Ft vs h21, device $1, \mathrm{VDS}=10 \mathrm{~V}, \mathrm{VGS}=-1 \mathrm{~V}$

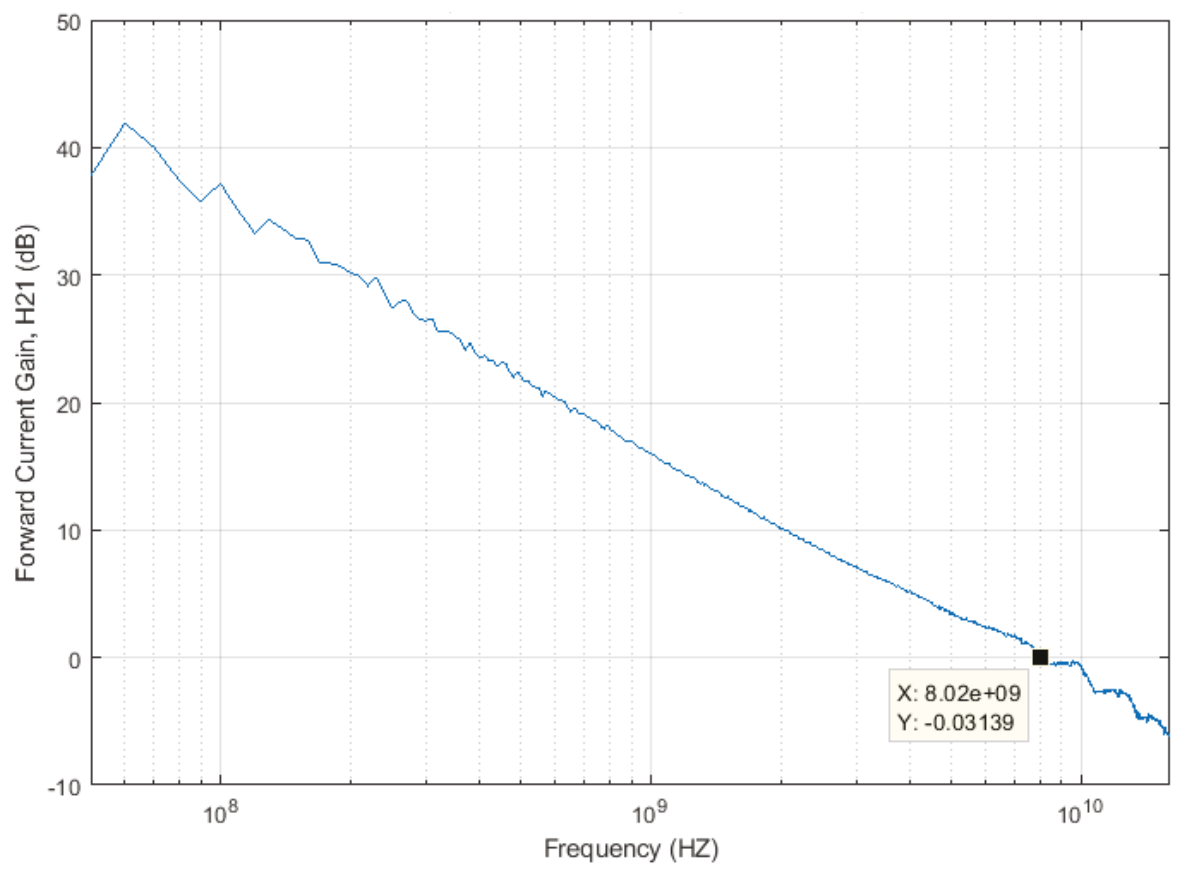

Figure 4.12: Ft vs h21, device $1, \mathrm{VDS}=10 \mathrm{~V}, \mathrm{VGS}=0 \mathrm{~V}$ 


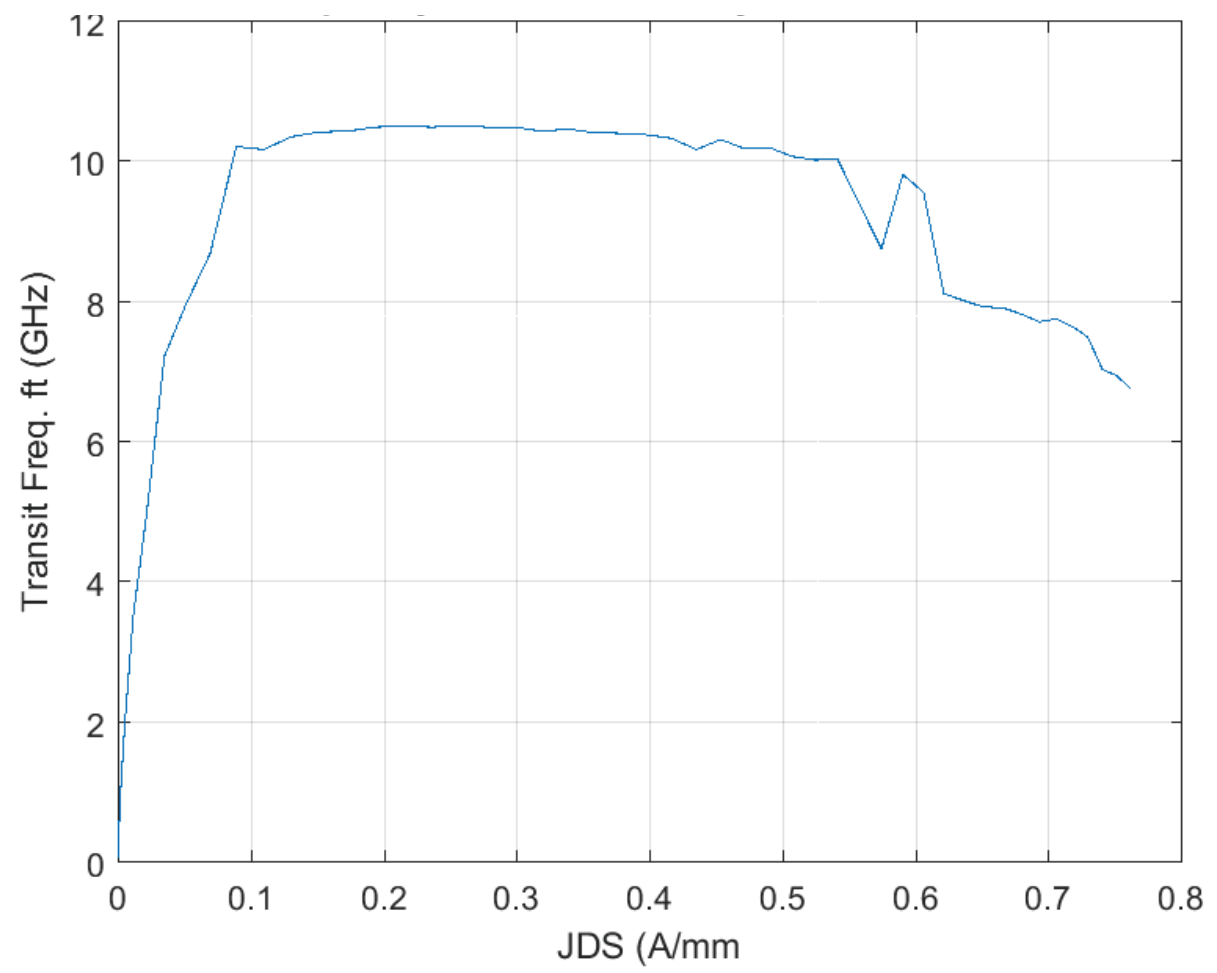

Figure 4.13: Ft vs IDS, device 1, VDS $=10 \mathrm{~V}$

The resulting $\mathrm{f}_{\mathrm{t}}$ for device 1 and device 2 are shown in Tables 4.3 to 4.6 for $\mathrm{V}_{\mathrm{DS}}$ of $5 \mathrm{~V}, 10 \mathrm{~V}, 15 \mathrm{~V}$ and $20 \mathrm{~V}$.

TABLE 4.3: Summary of $\mathrm{ft}$ results for $\mathrm{VDS}=5 \mathrm{~V}$

\begin{tabular}{|l|l|l|}
\hline JDS $(\mathrm{A} / \mathrm{mm})$ & Device $1 \mathrm{ft}(\mathrm{GHz})$ & Device 2 $(\mathrm{GHz})$ \\
\hline 0.1 & 10.1 & 13.3 \\
\hline 0.2 & 10.4 & 15.4 \\
\hline 0.3 & 10.4 & 15.6 \\
\hline 0.4 & 10.1 & 15.4 \\
\hline 0.5 & 8.2 & 15.3 \\
\hline 0.6 & 6.8 & 13.6 \\
\hline 0.7 & 2.9 & 10.4 \\
\hline
\end{tabular}

The obvious take away from the data is that there is a large difference in $f_{t}$ between the two devices. Device 1 exhibits a nominal $\mathrm{f}_{\mathrm{t}}$ of $10 \mathrm{GHz}$ whereas device 2 exhibits 
TABLE 4.4: Summary of ft results for VDS $=10 \mathrm{~V}$

\begin{tabular}{|l|l|l|}
\hline JDS $(\mathrm{A} / \mathrm{mm})$ & Device $1 \mathrm{ft}(\mathrm{GHz})$ & Device $2(\mathrm{GHz})$ \\
\hline 0.1 & 10.2 & 13.5 \\
\hline 0.2 & 10.6 & 15.6 \\
\hline 0.3 & 10.5 & 15.8 \\
\hline 0.4 & 10.4 & 15.7 \\
\hline 0.5 & 10.1 & 14.9 \\
\hline 0.6 & 9.6 & 13.4 \\
\hline 0.7 & 6.7 & 12.2 \\
\hline
\end{tabular}

TABLE 4.5: Summary of $\mathrm{ft}$ results for VDS $=15 \mathrm{~V}$

\begin{tabular}{|l|l|l|}
\hline JDS $(\mathrm{A} / \mathrm{mm})$ & Device $1 \mathrm{ft}(\mathrm{GHz})$ & Device $2(\mathrm{GHz})$ \\
\hline 0.1 & 10.4 & 13.5 \\
\hline 0.2 & 10.8 & 16.1 \\
\hline 0.3 & 10.6 & 16.0 \\
\hline 0.4 & 10.4 & 15.7 \\
\hline 0.5 & 10.2 & 15.3 \\
\hline 0.6 & 8.7 & 13.5 \\
\hline 0.7 & 7.8 & 11.5 \\
\hline
\end{tabular}

TABLE 4.6: Summary of $\mathrm{ft}$ results for VDS $=20 \mathrm{~V}$

\begin{tabular}{|l|l|l|}
\hline JDS $(\mathrm{A} / \mathrm{mm})$ & Device 1 ft $(\mathrm{GHz})$ & Device 2 $(\mathrm{GHz})$ \\
\hline 0.1 & 10.3 & 14.0 \\
\hline 0.2 & 10.8 & 15.9 \\
\hline 0.3 & 10.6 & 15.8 \\
\hline 0.4 & 10.3 & 14.1 \\
\hline 0.5 & 10.0 & 13.7 \\
\hline 0.6 & 8.0 & 13.1 \\
\hline 0.7 & 6.9 & 10.2 \\
\hline
\end{tabular}

a nominal $\mathrm{f}_{\mathrm{t}}$ of $15 \mathrm{GHz}$. The GaN500v3.0 design kit documentation specifies the $\mathrm{f}_{\mathrm{t}}$ of the device to be nominally $13 \mathrm{GHz}$.

The $\mathrm{f}_{\mathrm{t}}$ data for device 1 is plotted in Figure 4.14 and the $\mathrm{f}_{\mathrm{t}}$ data for device 2 is plotted in Figure 4.15. Comparing the two and disregarding the obvious difference in $\mathrm{f}_{\mathrm{t}}$, and can be observed that the $\mathrm{f}_{\mathrm{t}}$ for device 1 has less variation with $\mathrm{V}_{\mathrm{DS}}$ and also $\mathrm{J}_{\mathrm{DS}}$ than device 2 . 
The device ft was simulated using the design kit. The results are shown in Figure 4.16. The results indicate that the modeled device does indeed have a nominal $f_{t}$ of $13 \mathrm{GHz}$ agreeing with the specified value in the documentation.

The conclusion from this discussion is that the device's high frequency behaviour differs somewhat from the modeled behaviour and also varies from device to device. This should be considered when analyzing further results.

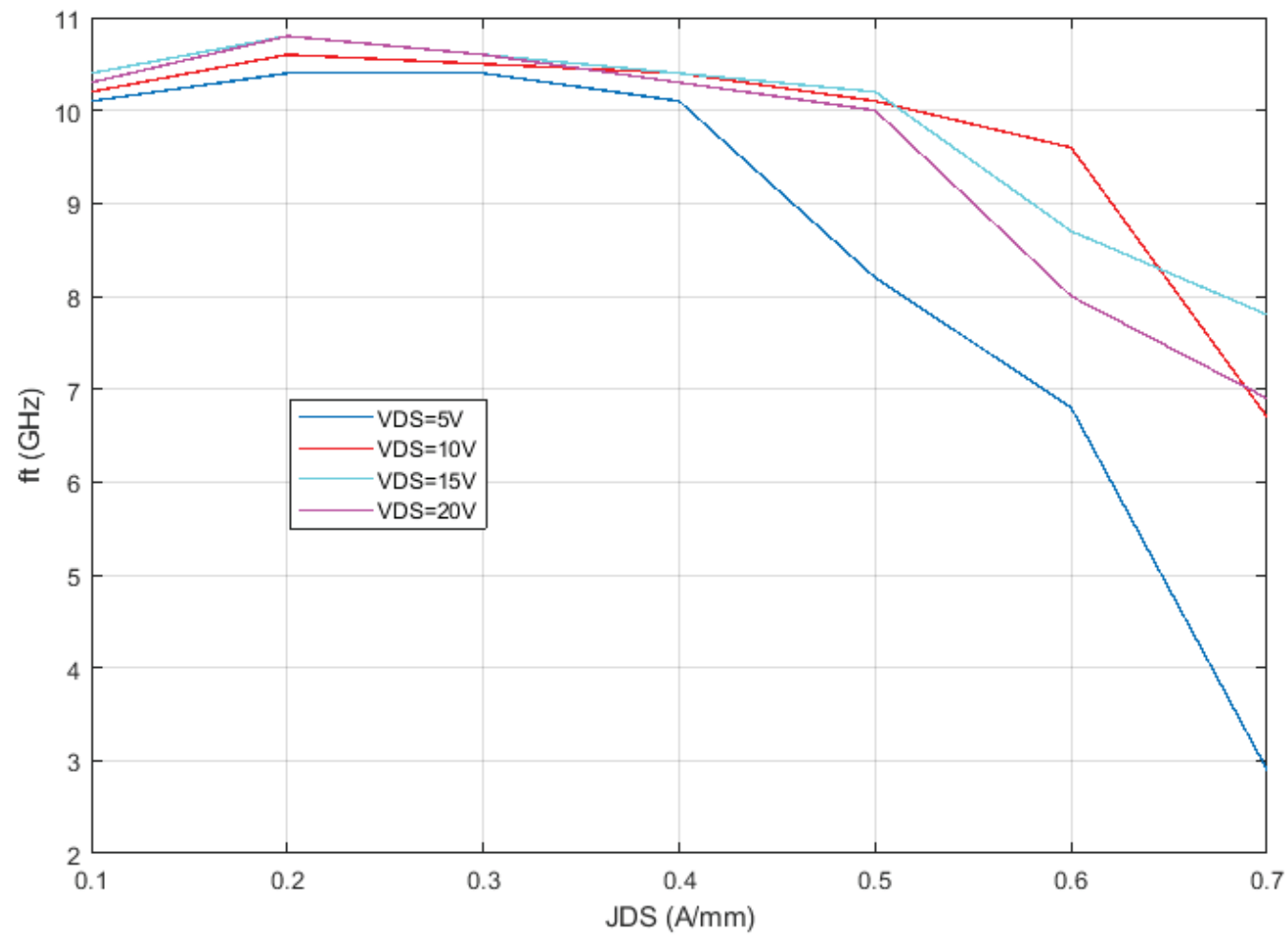

Figure 4.14: Ft vs. JDS, device 1, VDS $=5 \mathrm{~V}, 10 \mathrm{~V}, 15 \mathrm{~V}, 20 \mathrm{~V}$

Without measuring further devices and performing an in-depth analysis, a cause for this discrepancy between the devices and specified data cannot be explained. However, it should be noted that this is not a mature design kit so perhaps there are some processing issues that need to be resolved to achieve a more repeatable 
device. It is useful to bear these results in mind when analyzing results from the active inductor and bandpass filter.

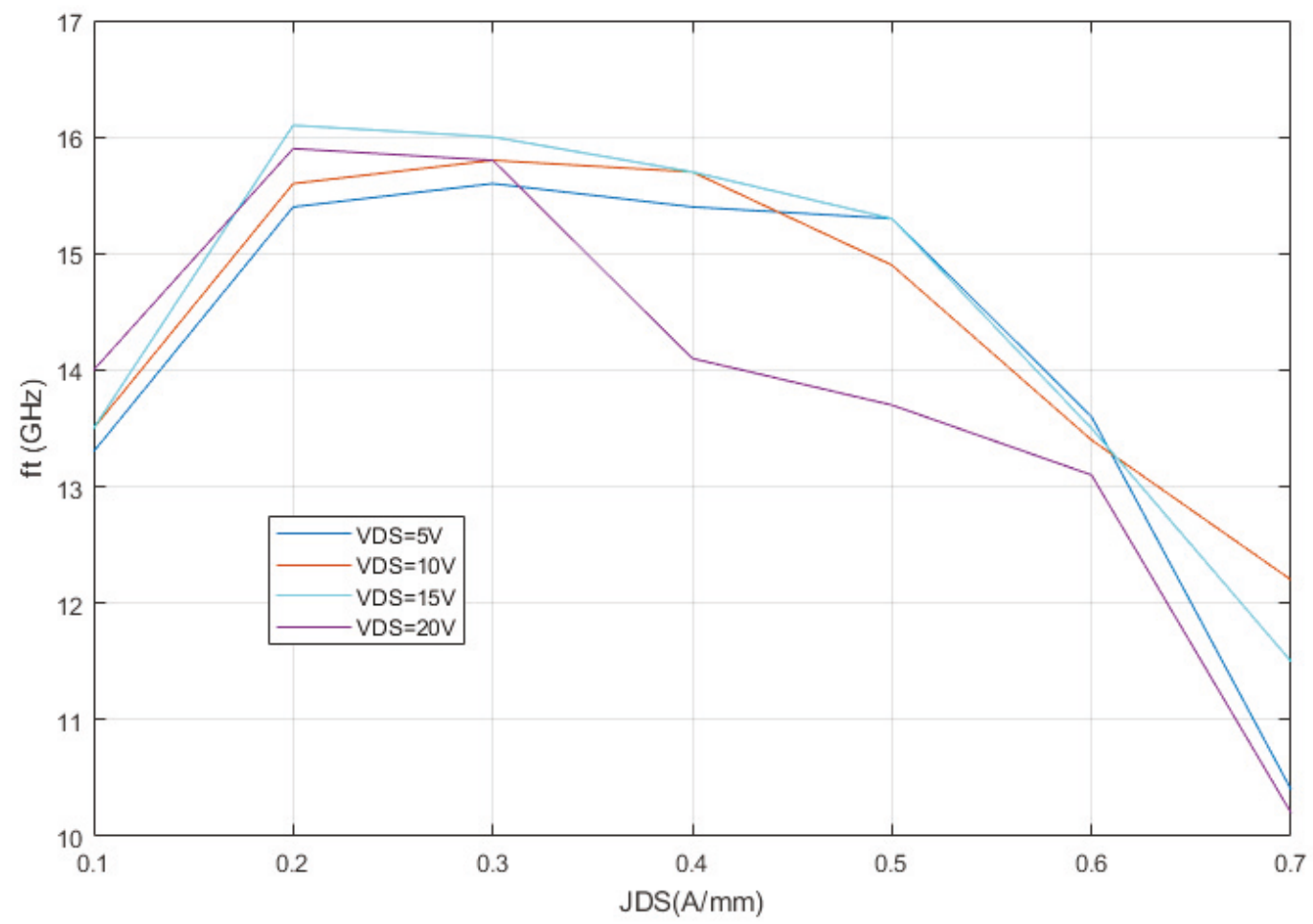

Figure 4.15: Ft vs. JDS, device 2, VDS $=5 \mathrm{~V}, 10 \mathrm{~V}, 15 \mathrm{~V}, 20 \mathrm{~V}$

\subsection{Active Inductor Characterization Setup and Processing}

\subsubsection{Setup and Post Processing}

The active inductor was placed on the test setup illustrated in Figure 4.2. Since the designed active inductor is a single ended device shunted to ground, only single port 


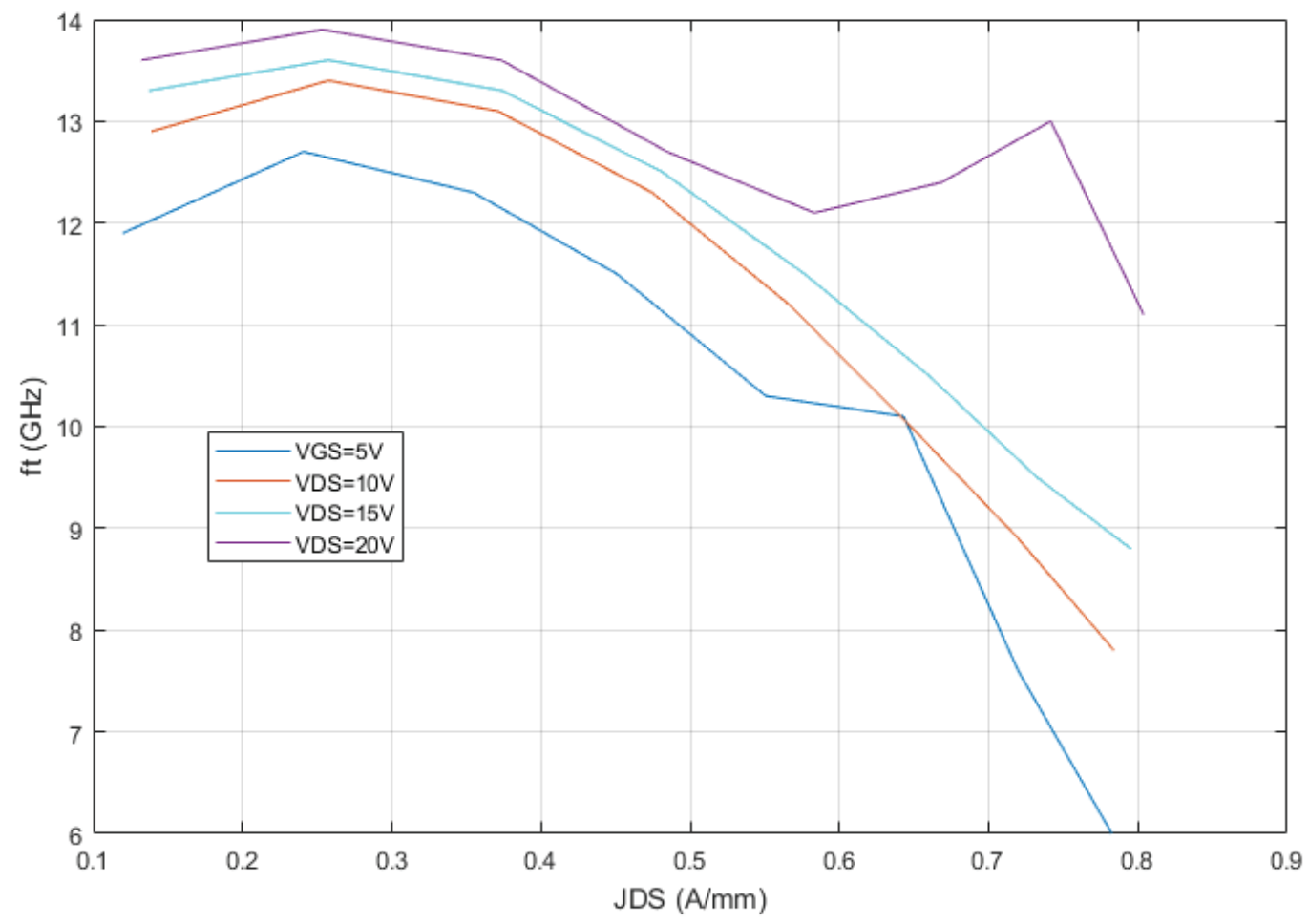

Figure 4.16: Ft vs. JDS, Simulated, VDS $=5 \mathrm{~V}, 10 \mathrm{~V}, 15 \mathrm{~V}, 20 \mathrm{~V}$

S-parameters were measured.

A VDD of $20 \mathrm{~V}$ was applied to the device for initial characterization. A software routine was written to sweep both VTUNE1 and VTUNE2 from -3V to 0V at a intervals of $0.05 \mathrm{~V}$. S11 was measured and stored automatically at each combination of VTUNE1, VTUNE2 resulting in a total of 3721 sweeps.

Examining such a large dataset manually would be too exhaustive. Therefore several mini data browser applications were created in Matlab allowing the user to view the device's calculated L and Q values as a function of either frequency, VTUNE1, or VTUNE2.

The viewer versus frequency is shown in 4.17 . 


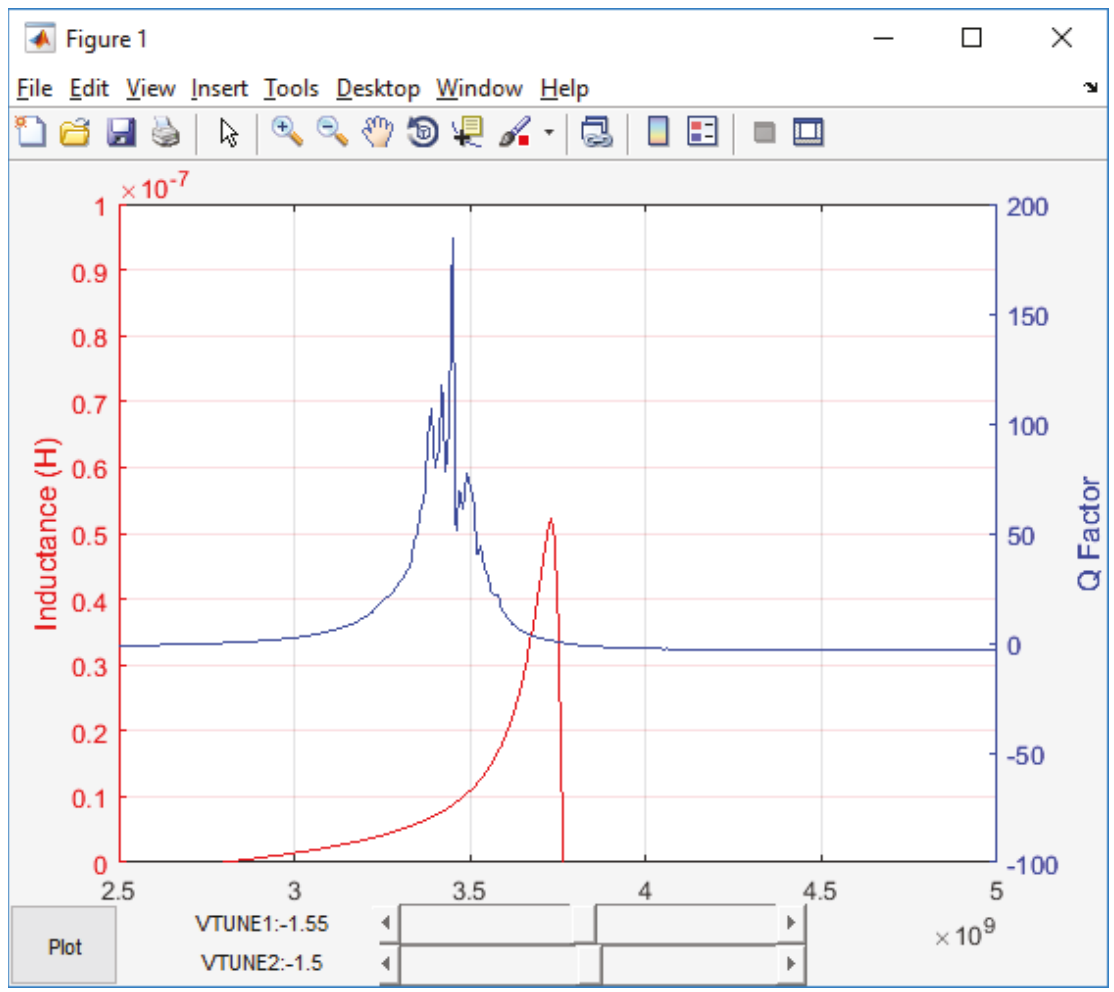

FiguRE 4.17: L and Q vs Frequency data viewer applet

The applet allows the user to select VTUNE1 and VTUNE2 via a pair of slider bars and automatically plots the corresponding L and Q data. Hitting the plot button allows several plots to be overlaid in an external figure window.

The data viewers for VTUNE1 and VTUNE2 as the independent variables are shown in Figures 4.18 and 4.19.

\subsubsection{Active Inductor Results}

Using the test setup and post-processing software described in the previous section, the active inductor was characterized across frequency, VTUNE1, and VTUNE2. 


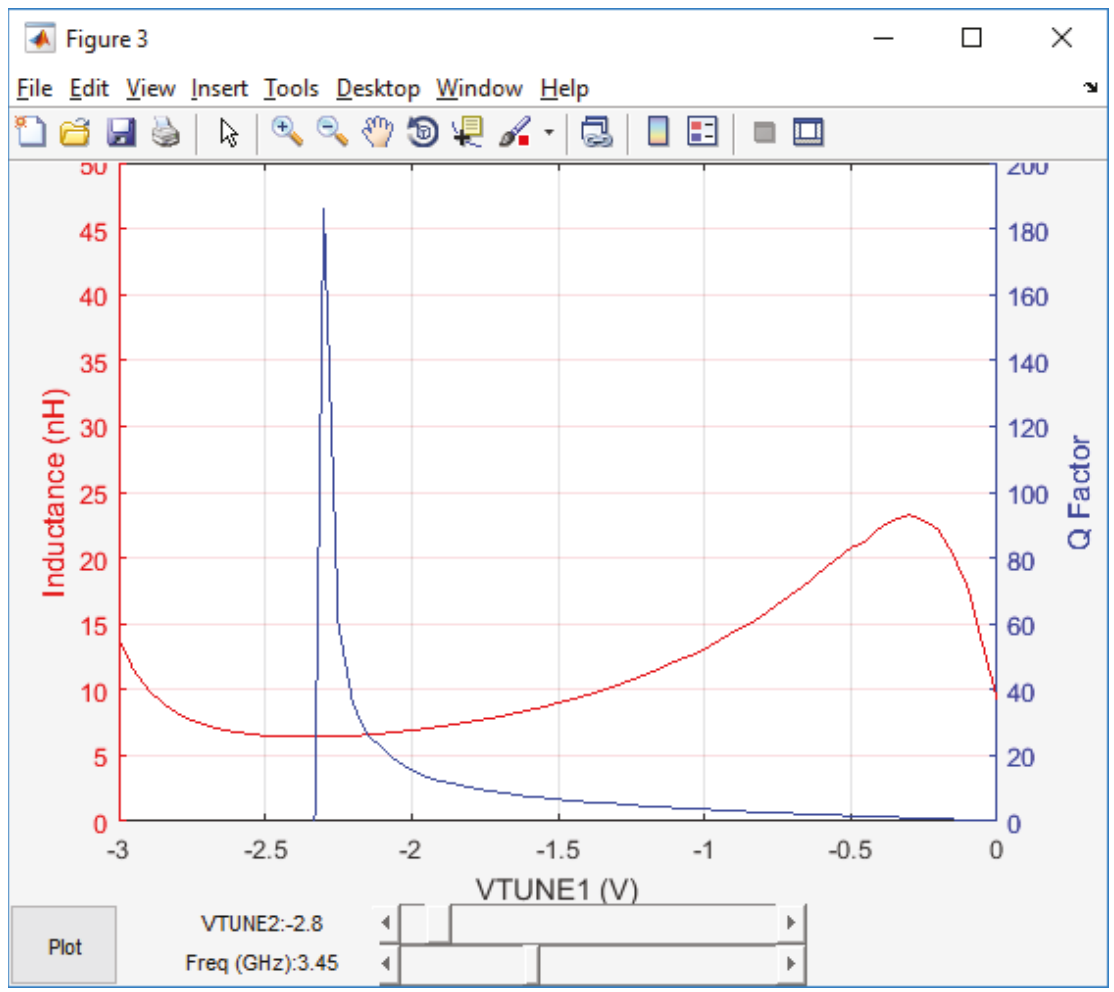

Figure 4.18: L and Q vs VTUNE1 data viewer applet

A typical frequency sweep is shown in Figure 4.20. This plot demonstrates a nominal frequency of operation of $3.5 \mathrm{GHz}$. The simulated nominal frequency of operation was found to be $5.4 \mathrm{GHz}$ as illustrated in Figure 3.15. The device's measured frequency response is therefore somewhat lower than its simulated response. A detailed comparison of simulated versus measured results are presented in Chapter 5.

The device exhibits a $\mathrm{Q}$ of 50 or greater over the range of $3.3 \mathrm{GHz}$ to $3.5 \mathrm{GHz}$ with a peak value just below 300 in this case. Other than the shift in frequency, this is agreeable with the simulated results

The frequency behaviour of the device is further illustrated in Figure 4.21 for various tuning combinations of VTUNE1 and VTUNE2. 


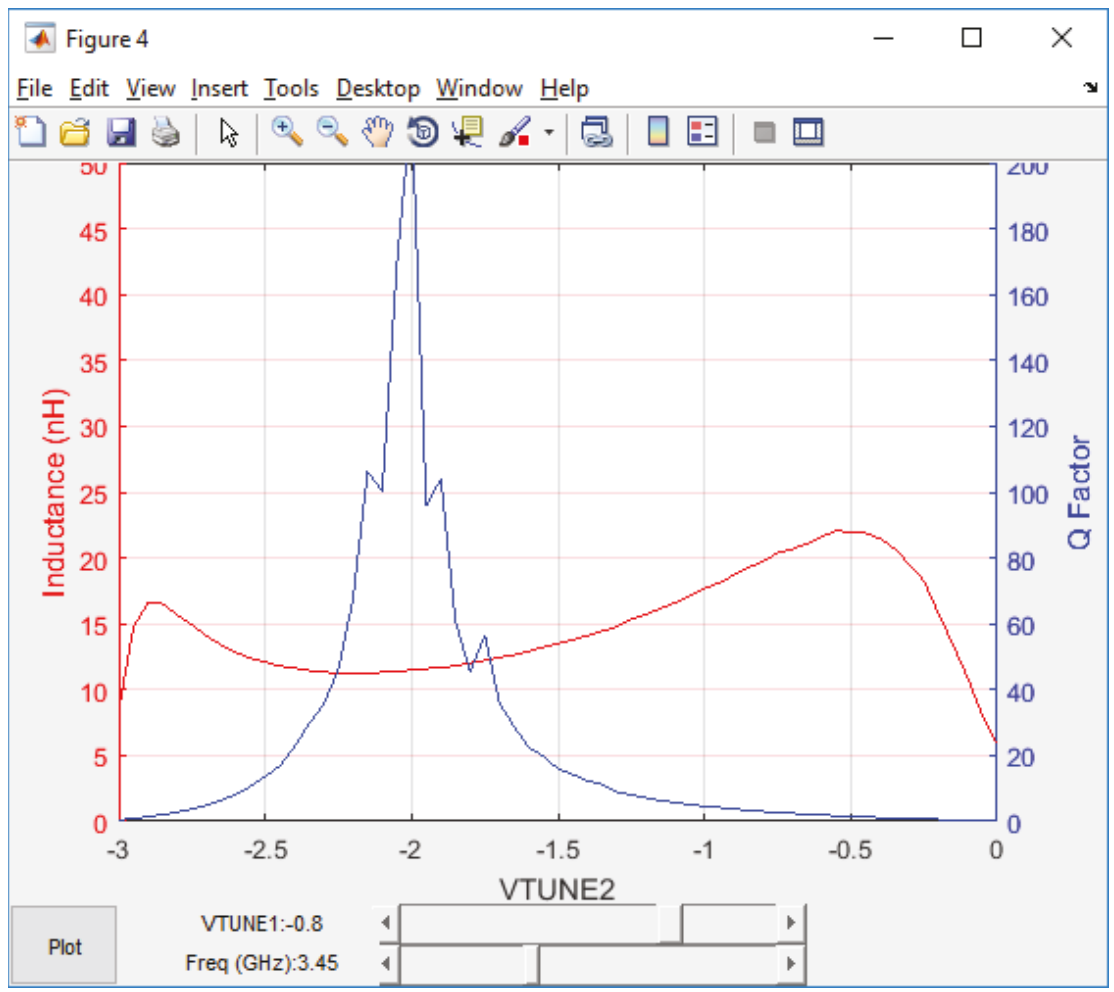

Figure 4.19: L and Q vs VTUNE2 data viewer applet

The active inductor was also characterized as a function of VTUNE1 with the frequency fixed at $3.5 \mathrm{GHz}$. The results are shown for various values of VTUNE2 in Figure 4.22. At this frequency, the inductance can be tuned from $8 \mathrm{nH}$ to approximately $20 \mathrm{nH}$ before the slope of the curve becomes steep. Note that by adjusting VTUNE2, the resulting change in the inductance is quite small in the useful region of VTUNE1 $=-2.0 \mathrm{~V}$ to $-1.0 \mathrm{~V}$. However altering VTUNE2 alters the position of the Q-Factor with respect to VTUNE1, therefore the inductance can be tuned with VTUNE1 and the Q-Factor can then be optimized using VTUNE2. This behaviour is an agreement with the simulated results in Section 3.

Similarly, the active inductance was characterized as a function of VTUNE2 with the frequency fixed at $3.5 \mathrm{GHz}$. The result is plotted in Figure 4.23. Comparing the inductance response to VTUNE2 vs the response to VTUNE1, there is still some 

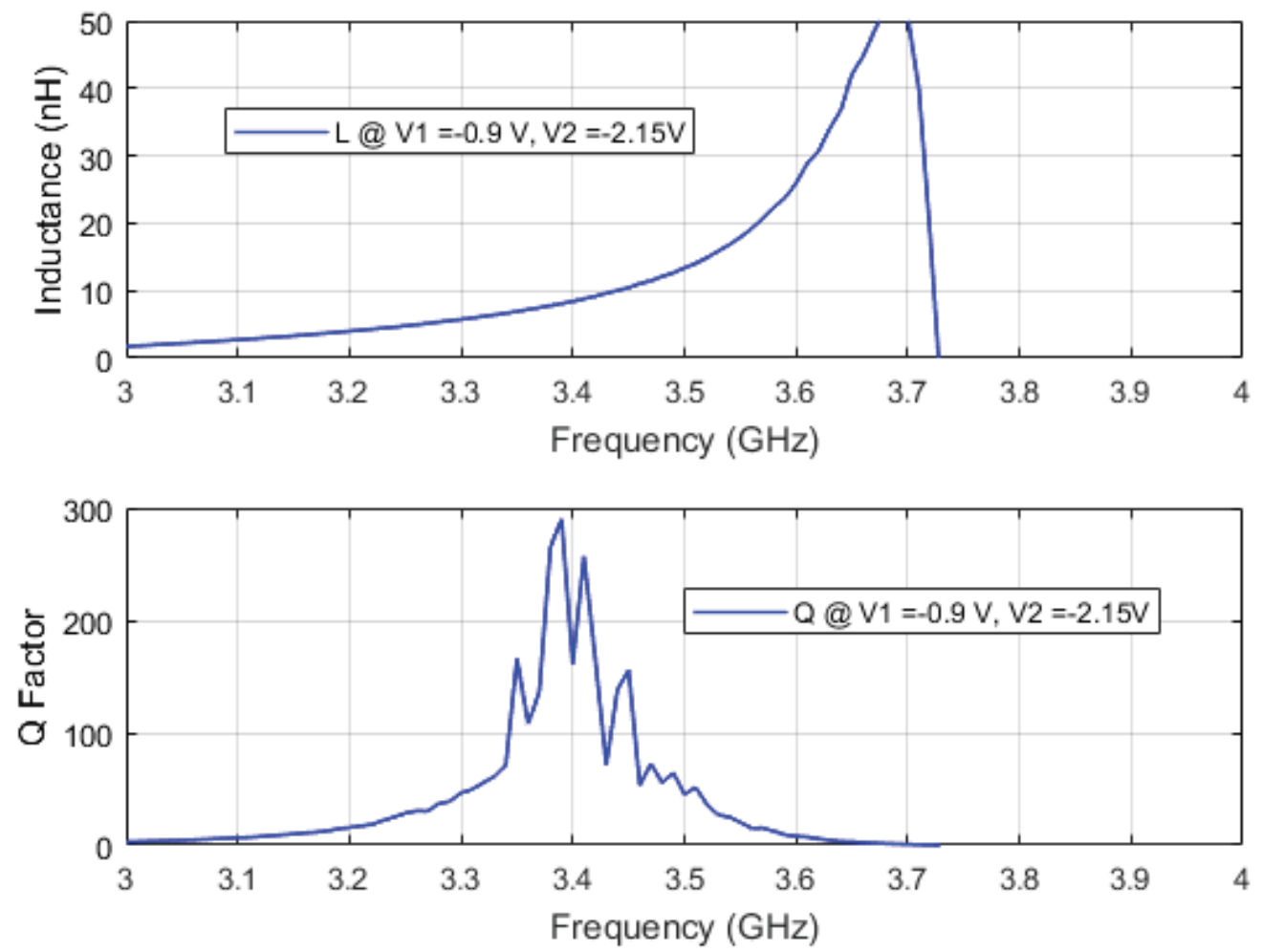

Figure 4.20: Typical Measured Active Inductor Response Across frequency

slight dependence but the dependence is not as strong. However, both VTUNE1 and VTUNE2 have a strong effect on the Q factor as was the case for the simulated results.

A second device was tested. Its frequency characteristic alongside the first device is shown in Figure 4.24. Device 1 shows a resonant frequency of $3.70 \mathrm{GHz}$ compared to $3.55 \mathrm{GHz}$ for device 2. At $3.3 \mathrm{GHz}$, device 1 has an inductance of $5 \mathrm{nH}$ whereas device 2 has an inductance of $10 \mathrm{nH}$. Considering the variance in the behaviour of the characterized FETs the slight variance in behaviour of the two active inductor devices is understandable. 

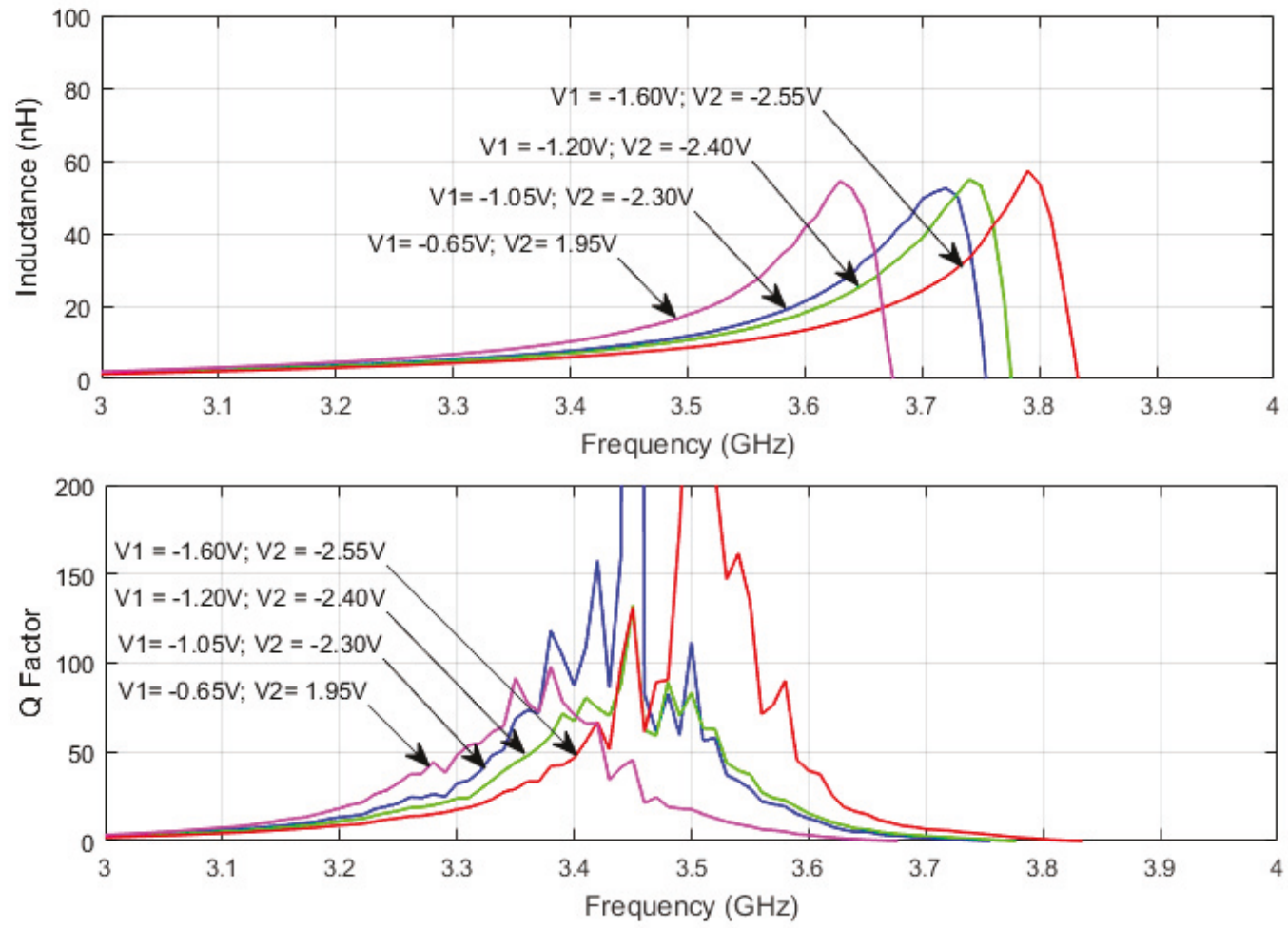

FiguRE 4.21: Measured Active Inductor parameters Across frequency

\subsubsection{Comparison of Active Inductor Simulated and Measured results}

The active inductor simulated and measured results are summarized in Table 4.7. Observing the simulated and measured plots, it is evident that both follow the same general trend. However, it is also notable that there is a large discrepancy in the frequency response. The simulated response demonstrates a nominal operating frequency of $5 \mathrm{GHz}$ whereas the measured response shows an operating frequency around 3.5 GHz. This discrepancy will be discussed in Section 5. 

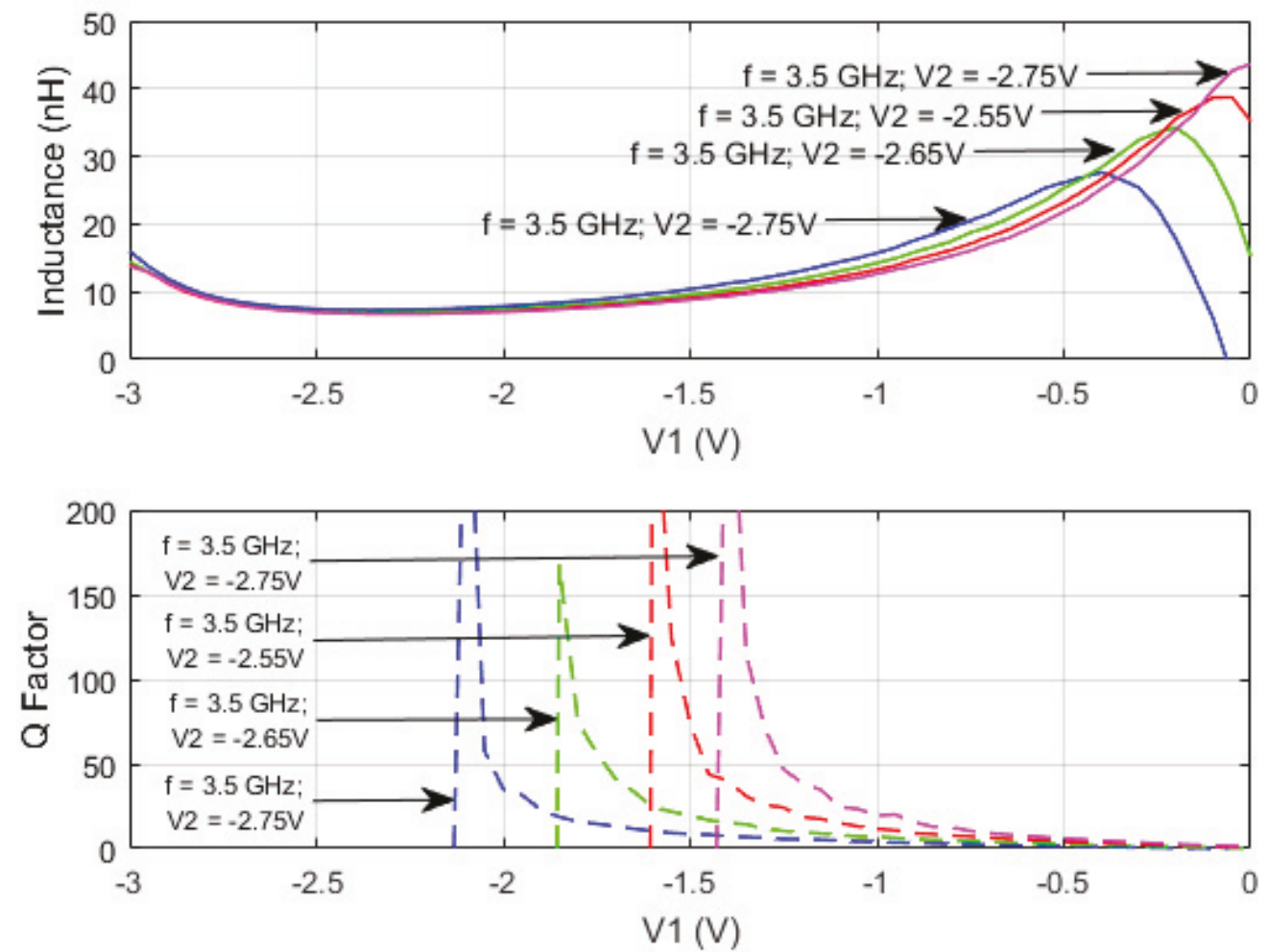

FiguRE 4.22: Measured Active Inductor Response Across VTUNE1 at $3.5 \mathrm{GHz}$

TABLE 4.7: Active Inductor Simulated Vs. Measured Results

\begin{tabular}{|l|l|l|l|l|}
\hline & $\begin{array}{l}\text { Inductive } \\
\text { Freq.y } \\
\text { Range }(\mathrm{GHz})\end{array}$ & $\begin{array}{l}\text { Nominal } \\
\text { Inductive } \\
\text { Freq. (GHz) }\end{array}$ & $\begin{array}{l}\text { Inductive Tuning } \\
\text { Range at } \\
\text { Nominal Freq. (nH) }\end{array}$ & $\begin{array}{l}\text { Q at } \\
\text { Nominal } \\
\text { Freq. }\end{array}$ \\
\hline Simulated & $3.5-6.5$ & 5 & $2.5-7$ & $>200$ \\
\hline Measured & $2.5-3.9$ & 3.5 & $8-20$ & $>200$ \\
\hline
\end{tabular}

\subsubsection{Bandpass Filter Results}

The bandpass filter was characterized using the test setup illustrated in Figure 4.2. Since the bandpass filter is a 2 port device, 2 port $\mathrm{S}$ parameters were obtained.

The forward gain S21 of the filter across frequency is shown in Figure 4.25 for various tuning values. 

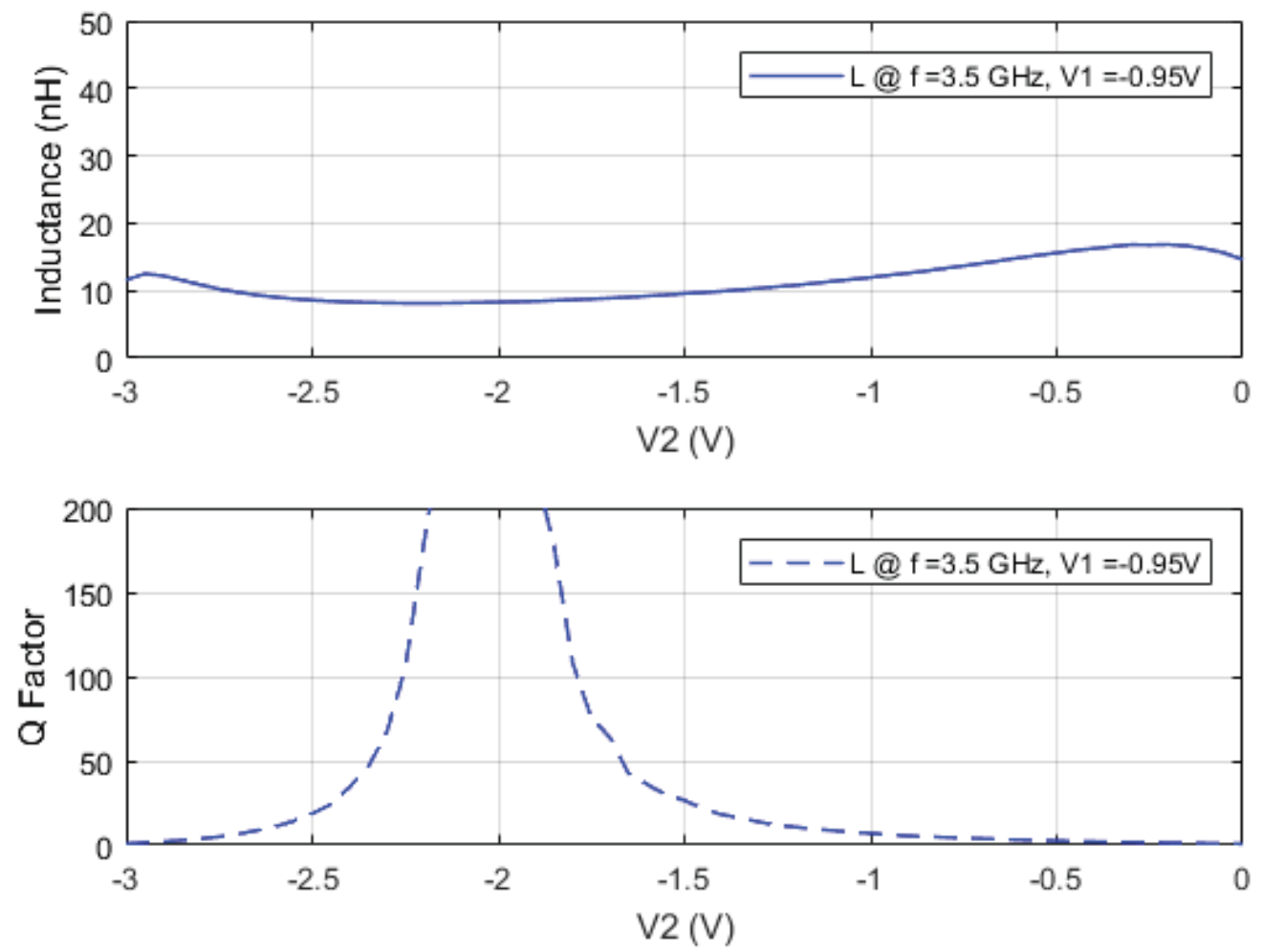

Figure 4.23: Measured Active Inductor Response Across VTUNE2 at $3.5 \mathrm{GHz}$

The filter exhibits a nominal center frequency of $3.5 \mathrm{GHz}$ with a tuning range of 3.0 $\mathrm{GHz}$ to $3.5 \mathrm{GHz}$ or $500 \mathrm{MHz}$ while maintaining a forward gain of between $3 \mathrm{~dB}$ and $6.8 \mathrm{~dB}$.

The simulated case exhibited a tuning range of $500 \mathrm{MHz}$ at a nominal frequency of $5 \mathrm{GHz}$ while maintaining a forward gain of $5 \mathrm{~dB}$.

It is difficult to identify specifically the cause of the variation in gain. It is noted that the gain increases with frequency. It is quite likely that this behaviour has something to do with the general frequency shift of the active inductor / filter response.

The response also demonstrates notches which exhibit a degree of tuning capability. Although these notches were not directly investigated they could be useful in certain 

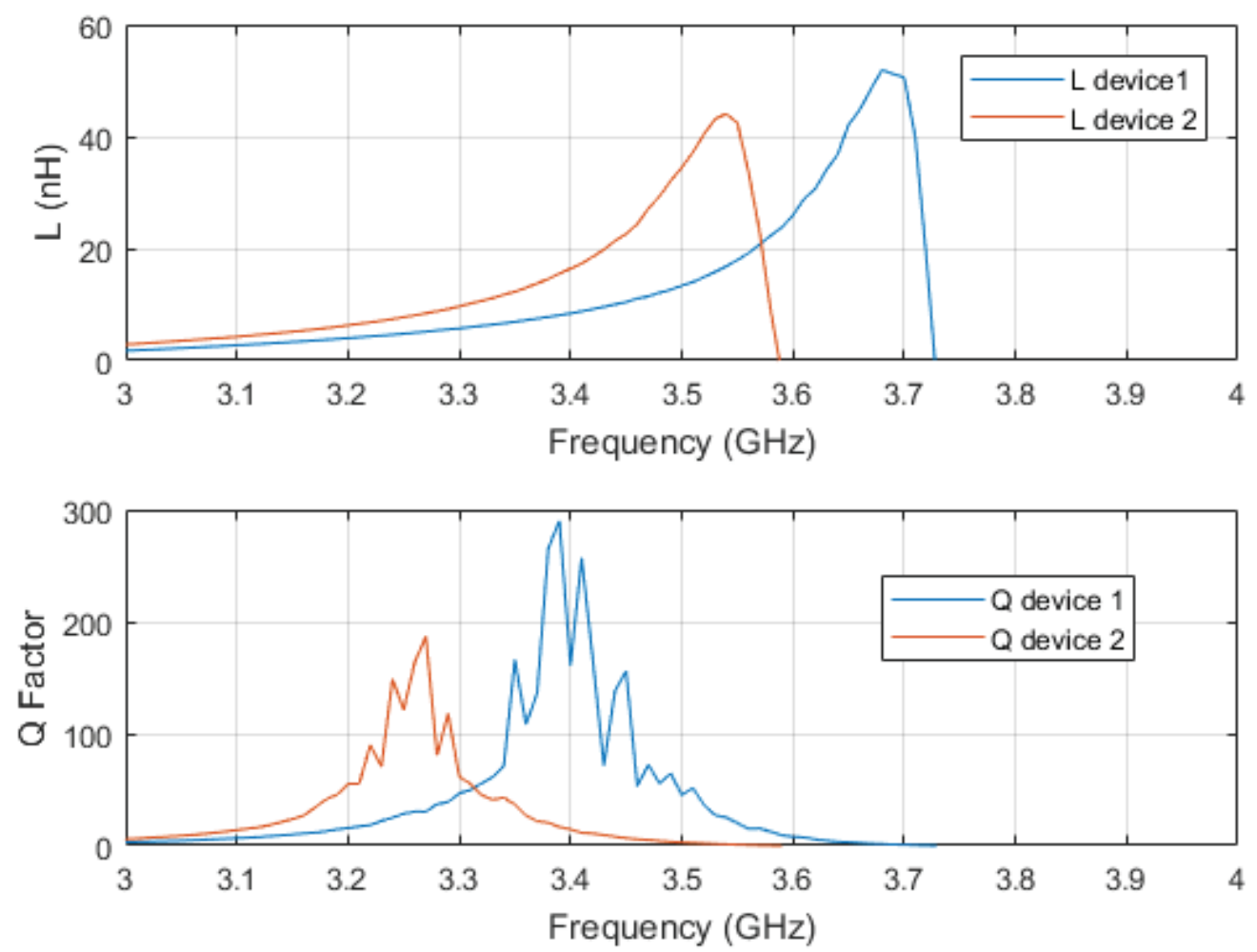

Figure 4.24: Measured Active Inductor Response of Device 1 and Device 2

applications where rejection of a certain frequency is required.

An enlargement of the passband, when tuned to $3.5 \mathrm{GHz}$, is shown in Figure 4.26. From the plot, the $3 \mathrm{~dB}$ bandwidth is measured to be $76 \mathrm{MHz}$ giving the filter a $\mathrm{Q}$ of 46 compared to a $\mathrm{Q}$ of 200 for the simulated case. The $10 \mathrm{~dB}$ bandwidth of the filter is measured to be $192 \mathrm{MHz}$.

The output $1 \mathrm{~dB}$ compression point was measured to be $-12 \mathrm{dBm}$ at a gain of $2 \mathrm{~dB}$. 


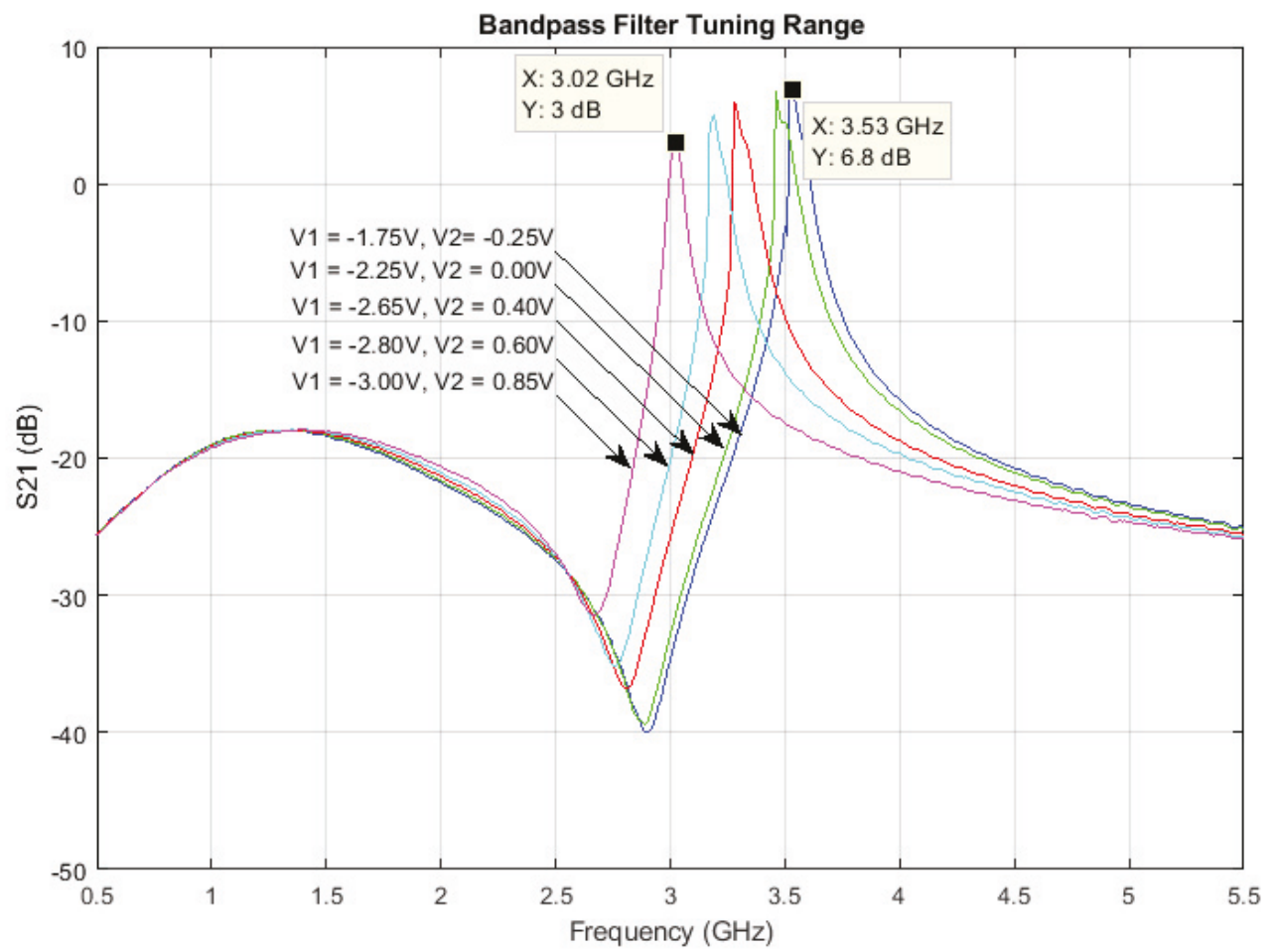

Figure 4.25: Measured Bandpass Filter Response

\subsubsection{Comparison of Bandpass Filter Simulated and Measured results}

The bandpass filter simulated and measured results are summarized in Table 4.8. Considering the standalone active inductor results demonstrated a nominal operating frequency of $5.0 \mathrm{GHz}$ simulated versus $3.5 \mathrm{GHz}$ measured, it is not surprising that the bandpass filter nominal frequency was also reduced to $3.5 \mathrm{GHz}$. Both filters demonstrate a $500 \mathrm{MHz}$ tuning range while maintaining a gain close to $5 \mathrm{~dB}$. Specifically, the simulated results demonstrated a tuning range of $4.5-5.0 \mathrm{GHz}$ whereas the measured results showed an actual tuning range of $3.0 \mathrm{GHz}-3.5 \mathrm{GHz}$.

The measured filter bandwidth (both $3 \mathrm{~dB}$ and $10 \mathrm{~dB}$ ) was larger than the simulated bandwidth. The increased $3 \mathrm{~dB}$ bandwidth for the measured case resulted in a $77 \%$ 


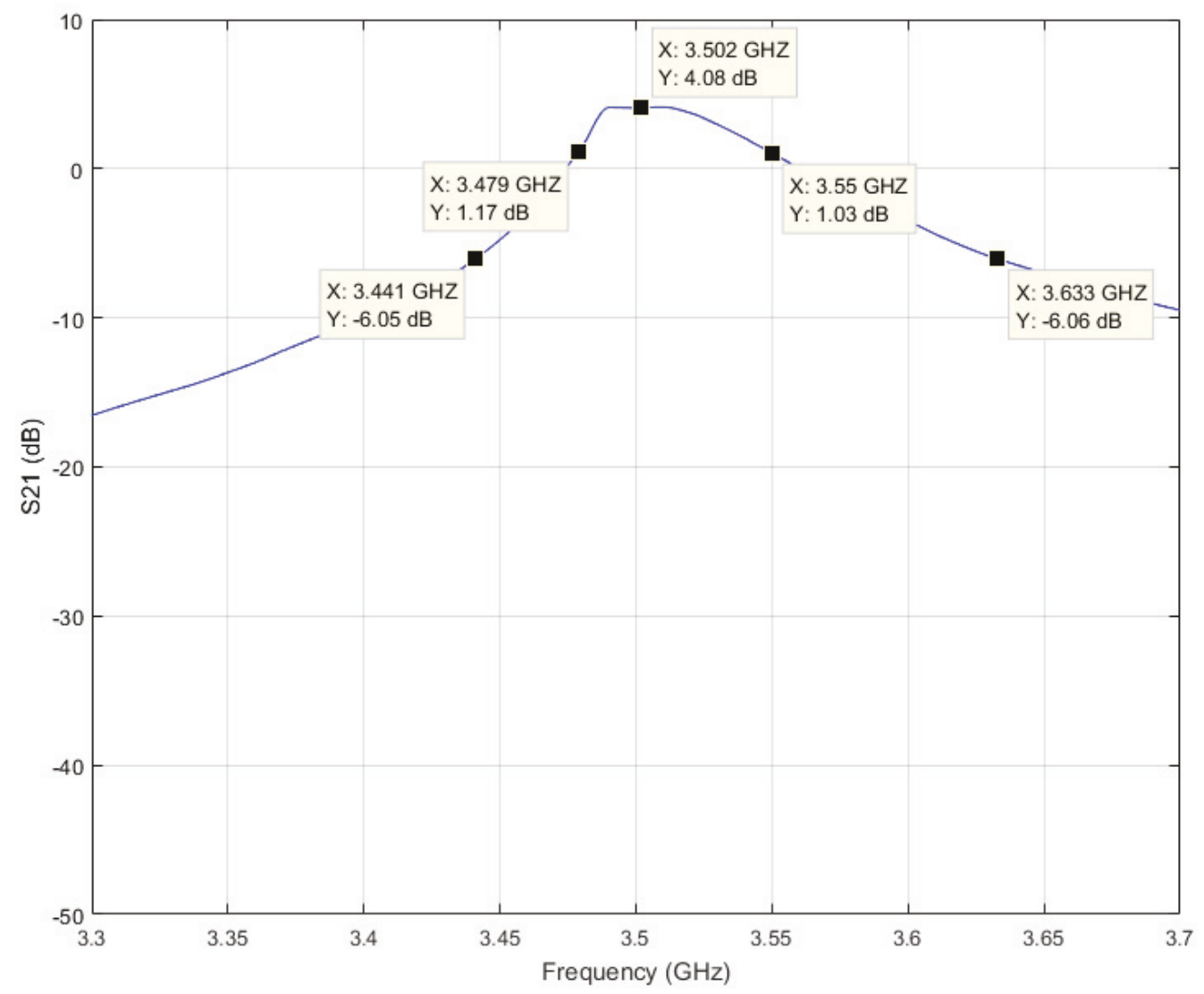

Figure 4.26: Measured Bandpass Filter Passband Width

reduction in the $\mathrm{Q}$ of the filter. Considering the general degradation in frequency performance of the active inductor this is unsurprising.

Regarding linearity, the output $1 \mathrm{~dB}$ compression point was $-14 \mathrm{dBm}$ (at a gain setting of $2 \mathrm{~dB}$ ) for the simulated case versus $-12 \mathrm{dBm}$ (at a gain setting of $2 \mathrm{~dB}$ ) for the measured case. 
TABLE 4.8: Bandpass Filter Simulated Vs. Measured Results

\begin{tabular}{|l|l|l|l|l|l|l|}
\hline & $\begin{array}{l}\text { Filter } \\
\text { Tuning } \\
\text { Range }(\mathbf{G H z})\end{array}$ & $\begin{array}{l}\text { Nominal } \\
\text { Gain } \\
(\mathbf{d B})\end{array}$ & $\begin{array}{l}\text { 3dB } \\
\text { BW } \\
(\mathbf{M H z})\end{array}$ & $\begin{array}{l}\text { 10dB } \\
\text { BW } \\
(\mathbf{M H z})\end{array}$ & Q & $\begin{array}{l}\text { Output } \\
\text { P1dB } \\
(\mathbf{d B m})\end{array}$ \\
\hline Simulated & $4.47-5.01$ & 5 & 25 & 77 & 200 & $\begin{array}{l}-14 @ \\
2 \text { dB gain }\end{array}$ \\
\hline Measured & $3.02-3.53$ & 5 & 76 & 192 & 46 & $\begin{array}{l}-12 @ \\
2 \text { dB gain }\end{array}$ \\
\hline
\end{tabular}

\subsection{Summary}

This chapter presented the measurement results of the fabricated circuits.

Firstly a discussion of the test setup was presented. This included a description of the hardware test setup along with a description of the software used to control the hardware setup and automate the measurement and data acquisition procedure.

The following section presented device characterization of Gan500 HEMTs. Firstly the device's DC behaviour was measured. Characteristic curves of $\mathrm{J}_{\mathrm{DS}}$ versus $\mathrm{V}_{\mathrm{DS}}$ for varying $V_{\text {GS }}$ were obtained for two samples. The results from the two samples demonstrated fairly close agreement, and agreed to $16 \%$ with the specified values given in the device manual.

The device was then characterized for AC performance. Specifically $f_{t}$ versus $J_{D S}$ was characterized. Again two samples were characterized. The first demonstrated a nominal $\mathrm{f}_{\mathrm{t}}$ of $10 \mathrm{GHz}$ whereas the second device demonstrated a nominal $\mathrm{f}_{\mathrm{t}}$ of $15 \mathrm{GHz}$. The documentation lists the $\mathrm{f}_{\mathrm{t}}$ as $13 \mathrm{GHz}$ nominally.

The active inductor was then characterized. The measurement results of the inductance and quality factor were presented as a function of frequency and also as a function of tuning voltages VTUNE1 and VTUNE2. The presented results followed the same general trend as the simulated results presented in chapter 3 , however the 
nominal frequency of operation of the device was measured to be $3.5 \mathrm{GHz}$ versus $5 \mathrm{GHz}$ for the simulated case.

The bandpass filter measurement results were then presented. Again the measured results demonstrated the same general trends as the simulated results, but again the nominal measured frequency was $3.5 \mathrm{GHz}$ versus $5 \mathrm{GHz}$ for the simulated case. The tuning range of the filter was measured to be $500 \mathrm{MHz}$ matching the simulated results.

The discrepancy in frequency will be discussed in the following chapter and an explanation with simulation results will be also be presented. 


\section{Chapter 5}

\section{Discussion}

\subsection{Frequency Discrepancy Discussion}

The general trend of the simulated and measured behaviour of the active inductor and bandpass filter are in agreement. However, there is $1.5 \mathrm{GHz}$ or $30 \%$ discrepancy in frequency. The initial design of the active conductor was design with HFETs with $f_{T}$ of $27 \mathrm{GHz}$. However during the course of the design, the design kit was updated and the HFET's $f_{T}$ was reduced to $13 \mathrm{GHz}$ thereby limiting the frequency behaviour of the device. The dielectric thickness between the two metal layers was also doubled shortly before manufacturing of the chip. It is possible that as a new process the thickness was not well modeled or controlled causing extra capacitance which would further degrade the frequency response of the device.

To model stray capacitance around the loop, an ideal $0.1 \mathrm{pF}$ capacitor was placed in shunt after each HFET as shown in 5.1. $0.1 \mathrm{pF}$ was selected since it is a fairly small value and would represent a roughly $20 \mathrm{X} 20 \mathrm{um}^{2}$ capacitor which could easily be added to existing lumped capacitors or stray capacitance if the dielectric height was not closely controlled.

The resulting simulation results are shown in Figure 5.2. When comparing to the measured results in Figure 4.21 the simulated and measured results both have nominal operating frequencies of $3.5 \mathrm{GHz}$ and nominal inductance values of $6 \mathrm{nH}$ versus $8 \mathrm{nH}$ respectively agreeing to within $25 \%$. 


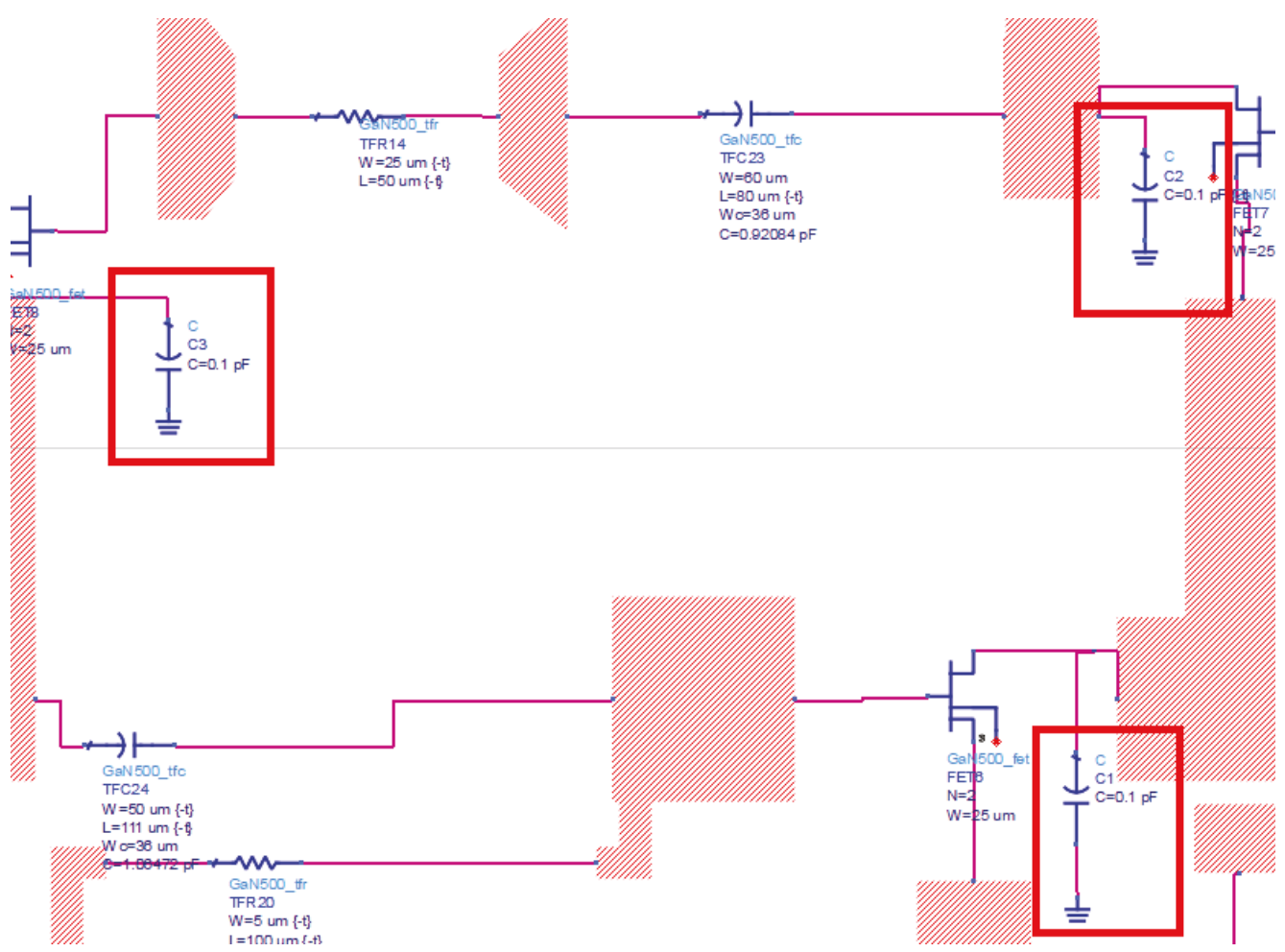

FiguRE 5.1: Active Inductor schematic modeling star capacitance

\subsection{Contributions}

This thesis aimed to provide the contributions set out in chapter 1. Namely, these were:

- The design and fabrication of a tunable active inductor in GaN with a nominal $5 \mathrm{GHz}$ operation.

- To demonstrate the use of the active inductor in a practical application. Specifically, the design and fabrication of a tunable bandpass filter in GaN incorporating the aforementioned active inductor. 


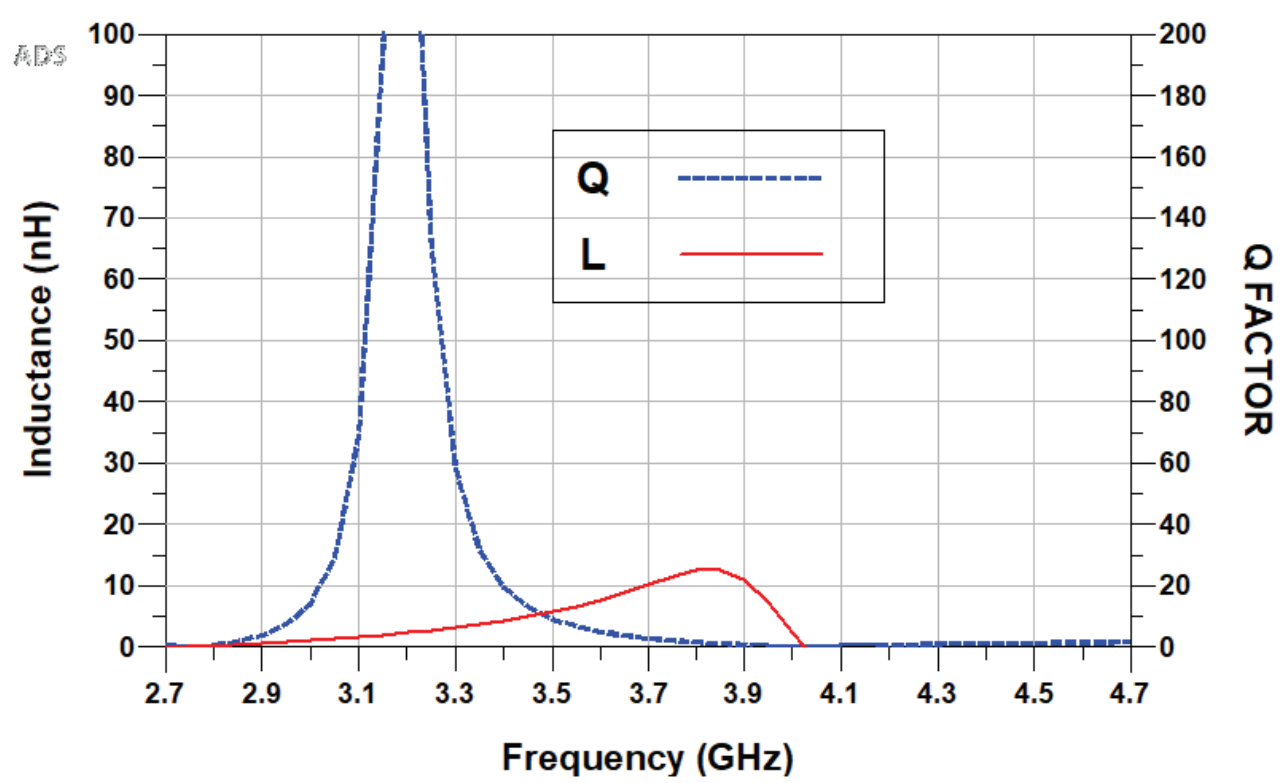

FIGURE 5.2: Simulation results modelling stray capacitance at bias inductor

- Characterization of design kit's active device using an automated setup. Characterization includes DC characterization and high frequency behaviour specifically $f_{t}$ versus current density.

The thesis was successful in achieving all three contributions.

Although demonstrating a high frequency dependence and a discrepancy between the simulated and measured frequency of operation, the active inductor did demonstrate the ability to be tuned while simultaneously achieving high Q values of several hundred. The high $\mathrm{Q}$ was obtained as a result of the negative feedback behaviour of the active inductor canceling the high contact resistance of the transistors. Frequency performance aside, this proved that GaN has the potential to form high quality active inductors.

Furthermore, the practical application of the active inductor was demonstrated in the design and fabrication of a tunable band pass filter. Here the resonant behaviour of 
the active inductor was exploited resulting in a high order filter with a high frequency roll-off. The band pass filter achieved a tuning range of $500 \mathrm{MHz}$ while maintaining a gain around $5 \mathrm{~dB}$. The linearity of the device was not as high as would be desired (nominally $-15 \mathrm{~dB}$ output $1 \mathrm{~dB}$ compression point), however, it would still be of practical use within a receiver where typical power levels would be expected to be considerably less than the measured compression point.

The device characterization was useful in comparing the active device's measured behaviour to its modelled behaviour. The characterization was successful in demonstrating that the active device's measured DC behaviour was consistent with the device's modelled DC behaviour. However, the characterization also showed that there was a discrepancy between the measured $f_{t}$ of the device and the modelled $f_{t}$. The modelled behaviour demonstrated an $\mathrm{f}_{\mathrm{t}}$ of $13 \mathrm{GHz}$ whereas the measured $\mathrm{f}_{\mathrm{t}}$ of two devices were $10 \mathrm{GHz}$ and $15 \mathrm{GHz}$.

\subsection{Future Work}

The focal point of future work will be on improving the frequency response of the Active Inductor.

Regardless of the discrepancy between the simulated and measured results, both sets of results demonstrated an inductance highly dependent upon frequency with sharp resonance. Although this behaviour was exploited in the design of the band pass filter, in general, this behaviour is undesirable. Several factors could be at fault for this behaviour such as frequency response of the active devices, stray capacitance, circuit topology, self-resonance of biasing inductor. 
A second area that needs to be investigated further and improved is the linearity of the devices. The bandpass filter showed output $1 \mathrm{~dB}$ compression points of $-15 \mathrm{dBm}$ depending on the gain setting. Observing how sensitive the active inductor behaviour is to tuning voltage, it is then no surprise that low level input signals therefore alter the device behaviour. A similar issue was encountered in work on varactors in GaN here at Carleton which also resulted in low linearity [24].

The circuit was extremely sensitive to parameter variation which in turn probably makes it sensitive to process variation. It would be beneficial to make the performance more robust in terms in parameter variation.

Finally replacing the bias inductor with active biasing would greatly reduce the area of the device and perhaps also improve the frequency performance considering the self-resonance of the fixed inductor. 


\section{Bibliography}

[1] A. Elasser and T. P. Chow, "Silicon carbide benefits and advantages for power electronics circuits and systems," Proceedings of the IEEE, vol. 90, no. 6, pp. 969-986, Jun. 2002.

[2] P. G. Neudeck, R. S. Okojie, and L.-Y. Chen, "High-temperature electronics - a role for wide bandgap semiconductors?" Proceedings of the IEEE, vol. 90, no. 6, pp. 1065-1076, Jun. 2002.

[3] J. A. Cooper and A. Agarwal, "SiC power-switching devices-the second electronics revolution?" Proceedings of the IEEE, vol. 90, no. 6, pp. 956-968, Jun. 2002.

[4] M. Ostling, R. Ghandi, and C. M. Zetterling, "SiC power devices; Present status, applications and future perspective," in 2011 IEEE 23rd International Symposium on Power Semiconductor Devices and ICs, May 2011, pp. 10-15.

[5] C. H. Oxley and M. J. Uren, "Measurements of unity gain cutoff frequency and saturation velocity of a GaN HEMT transistor," IEEE Transactions on Electron Devices, vol. 52, no. 2, pp. 165-169, Feb. 2005.

[6] K. Bensen. (2017). "GaN Breaks Barriers: RF Power Amplifiers Go Wide and High", [Online]. Available: http://www. analog.com/en/analog-dialogue/ articles / rf - power-amplifiers-go-wide-and-high . html (visited on $05 / 02 / 2018)$.

[7] C. F. Campbell, A. Balistreri, M. Y. Kao, D. C. Dumka, and J. Hitt, "GaN Takes the Lead," IEEE Microwave Magazine, vol. 13, no. 6, pp. 44-53, Sep. 2012.

[8] R. J. Trew, G. L. Bilbro, W. Kuang, Y. Liu, and H. Yin, "Microwave AlGaN / GaN HFETs," IEEE Microwave Magazine, vol. 6, no. 1, pp. 56-66, Mar. 2005. 
[9] Y. Nakasha, S. Masuda, K. Makiyama, T. Ohki, M. Kanamura, N. Okamoto, T. Tajima, T. Seino, H. Shigematsu, K. Imanishi, T. Kikkawa, K. Joshin, and N. Hara, "E-Band 85mW Oscillator and 1.3W Amplifier ICs Using 0.12 um GaN HEMTs for Millimeter-Wave Transceivers," in 2010 IEEE Compound Semiconductor Integrated Circuit Symposium (CSICS), Oct. 2010, pp. 1-4.

[10] A. Brown, K. Brown, J. Chen, K. C. Hwang, N. Kolias, and R. Scott, "W-band GaN Power Amplifier MMICs," in 2011 IEEE MTT-S International Microwave Symposium, Jun. 2011, pp. 1-4.

[11] T. Ross, G. Cormier, and K. Hettak, "Gallium Nitride Hybrid Microwave Circuits for Low-Noise Applications," in 2010 14th International Symposium on Antenna Technology and Applied Electromagnetics the American Electromagnetics Conference, Jul. 2010, pp. 1-4.

[12] S. Colangeli, A. Bentini, W. Ciccognani, E. Limiti, and A. Nanni, "GaN-Based Robust Low-Noise Amplifiers," IEEE Transactions on Electron Devices, vol. 60, no. 10 , pp. 3238-3248, Oct. 2013, ISSN: 0018-9383.

[13] J. M. Carroll, "Using GaN FETs for High Power RF Switches," in 2008 IEEE Compound Semiconductor Integrated Circuits Symposium, Oct. 2008, pp. 1-4.

[14] G. Polli, M. Palomba, S. Colangeli, M. Vittori, and E. Limiti, "High PowerHandling GaN Switch for S-band Applications," in 2017 Integrated Nonlinear Microwave and Millimetre-wave Circuits Workshop (INMMiC), Apr. 2017, pp. $1-4$.

[15] RFMD. (2012). "GaN Switches Enable Hot Switching at Higher Power", [Online]. Available: http : //www . microwavejournal . com/articles /483-ganswitches-enable-hot-switching-at-higher-power (visited on 09/10/2018). 
[16] V. Egorkin, A. Zaitsev, V. Garmash, O. Zagidullina, and A. Negencev, "Digital Circuits Based on GaN HEMTs with Threshold Voltage Adjusted by Fluorine Plasma Treatment," in 2017 IEEE Conference of Russian Young Researchers in Electrical and Electronic Engineering (EIConRus), Feb. 2017, pp. 1131-1134.

[17] A. V. Jawake, K. S. Bhosale, H. S. Borse, S. R. Patil, S. R. Aher, and G. C. Patil, "Comparative Analysis of GaN-on-3CSiC and Conventional Si MOSFET for Digital Integrated Circuits," in 2015 Annual IEEE India Conference (INDICON), Dec. 2015, pp. 1-4.

[18] Ultra cmos digitally tunable capacitor, PE64904, pSemi Corp., 2018.

[19] M. Marso, M. Wolter, J. Bernat, P. Javorka, A. Fox, and P. Kordos, "Varactor Diodes Based on an AlGaN/GaN HEMT Layer Structure," in 2001 International Symposium on Electron Devices for Microwave and Optoelectronic Applications. EDMO 2001, 2001, pp. 37-42.

[20] C. Y. Jin, J. Y. Wang, M. Fang, J. B. Cai, Y. Liu, Z. Yang, B. Zhang, W. G. Wu, and J. C. Zhang, "A GaN-Based Metal-Semiconductor-Metal Planar InterDigitated Varactor," in 2012 IEEE 11th International Conference on SolidState and Integrated Circuit Technology, Oct. 2012, pp. 1-3.

[21] A. Hamdoun, "Design of GaN-Based Microwave Components and Application to Novel High Power Reconfigurable Antennas," PhD thesis, Carleton University, 2016.

[22] W. Lu, L. Wang, S. Gu, D. P. R. Aplin, D. M. Estrada, P. K. L. Yu, and P. M. Asbeck, "InGaN/GaN Schottky Diodes With Enhanced Voltage Handling Capability for Varactor Applications," IEEE Electron Device Letters, vol. 31, no. 10, pp. 1119-1121, Oct. 2010. 
[23] W. Lu, L. Wang, S. Gu, P. M. Asbeck, and P. K. L. Yu, "Critical Design Considerations for GaN-Based Microwave Power Varactors," in 2012 IEEE International Conference on Electron Devices and Solid State Circuit, Dec. 2012, pp. 1-4.

[24] A. Hamdoun, L. Roy, M. Himdi, and O. Lafond, "Characterization of GaNBased HEMTs as Varactor Diode Devices," in 2015 10th European Microwave Integrated Circuits Conference (EuMIC), Sep. 2015, pp. 417-420.

[25] F. Yuan, CMOS Active Inductors and Transformers, 1st ed. Springer, 2008, pp. 21-27.

[26] T. Yanagisawa and Y. Kawashima, "Active Gyrator," Electronics Letters, vol. 3, no. 3, pp. 105-107, Mar. 1967.

[27] E. Vanabelle, P. Descamps, and J. Vindevoghel, "Narrowband MMIC Filters Using Active GaAs Inductors. Application to HDTV," in 1998 28th European Microwave Conference, vol. 1, Oct. 1998, pp. 368-372.

[28] C. Leifso, J. W. Haslett, and J. G. McRory, "Monolithic Tunable Active Inductor with Independent Q Control," IEEE Transactions on Microwave Theory and Techniques, vol. 48, no. 6, pp. 1024-1029, Jun. 2000.

[29] D. M. Zaiden, J. E. Grandfield, T. M. Weller, and G. Mumcu, "Compact and Wideband MMIC Phase Shifters Using Tunable Active Inductor-Loaded AllPass Networks," IEEE Transactions on Microwave Theory and Techniques, vol. 66, no. 2, pp. 1047-1057, Feb. 2018.

[30] H. Xiao, R. Schaumann, W. R. Daasch, P. K. Wong, and B. Pejcinovic, "A Radio-Frequency CMOS Active Inductor and its Application in Designing HighQ Filters," in 2004 IEEE International Symposium on Circuits and Systems, vol. 4, May 2004, pp. 197-200. 
[31] J. Xu, C. E. Saavedra, and G. Chen, "An Active Inductor-Based VCO With Wide Tuning Range and High DC-to-RF Power Efficiency," IEEE Transactions on Circuits and Systems II: Express Briefs, vol. 58, no. 8, pp. 462-466, Aug. 2011.

[32] R. Srivastava and S. Mangesh, "Active Inductor Based VCO for High Tuning Range," International Journal of Applied Information Systems, vol. 4, no. 3, pp. 29-33, Sep. 2012.

[33] J. Manjula and S. Malarvizh, "Low Power Low Noise Tunable Active Inductor for Narrow Band LNA Design," International Journal of Computer Applications, vol. 47, no. 17, pp. 39-43, Jun. 2012.

[34] R. Kumar, D. Sachan, S. S. Yadav, A. K. Sihara, and P. K. Misra, "Design of Active Inductor at $2.4 \mathrm{GHz}$ Frequency Using 180 nm CMOS Technology," in 2017 4th IEEE Uttar Pradesh Section International Conference on Electrical, Computer and Electronics (UPCON), Oct. 2017, pp. 477-481.

[35] J. Manjula, P. S. Krishna, and D. Rubeena, "Design and performance analysis of active inductor based reconfigurable regulated cascode lna for tunable rf front end," in 2017 International Conference on Communication and Signal Processing (ICCSP), Apr. 2017, pp. 222-227.

[36] V. Kumar, R. Mehra, and A. Islam, "A 2.5 GHz Low Power, High-Q, Reliable Design of Active Bandpass Filter," IEEE Transactions on Device and Materials Reliability, vol. 17, no. 1, pp. 229-244, Mar. 2017.

[37] Gallium Nitride MMIC Foundry Design Manual: NRC GaN500, 3.01, National Research Council Canada, 2014.

[38] N. M. Shrestha, Y. Li, and E. Y. Chang, "Simulation Study on Electrical Characteristic of AlGaN/GaN High Electron Mobility transistors with AlN Spacer Layer," Japanese Journal of Applied Physics, vol. 53, pp. 1-8, Feb. 2014. 
[39] N. Tiwari, S. K. Gupta, and S. N. Mishra, "Impact of AlGaN Barrier Layer on DC and AC Characteristics of AlxGa1-xN/GaN MOSHEMT," in 2017 1st International Conference on Electronics, Materials Engineering and NanoTechnology (IEMENTech), Apr. 2017, pp. 1-4.

[40] S. Faramehr, P. Igifffdfffd, and K. Kalna, "Modelling and Optimization of GaN Capped HEMTs," in The Tenth International Conference on Advanced Semiconductor Devices and Microsystems, Oct. 2014, pp. 1-4. 


\section{Appendix A}

This appendix contains device characterization plots referred to in Section 4.2

\section{A.1 Device 1}

Figure A.1: Ft vs IDS, device 1, VDS $=5 \mathrm{~V}$

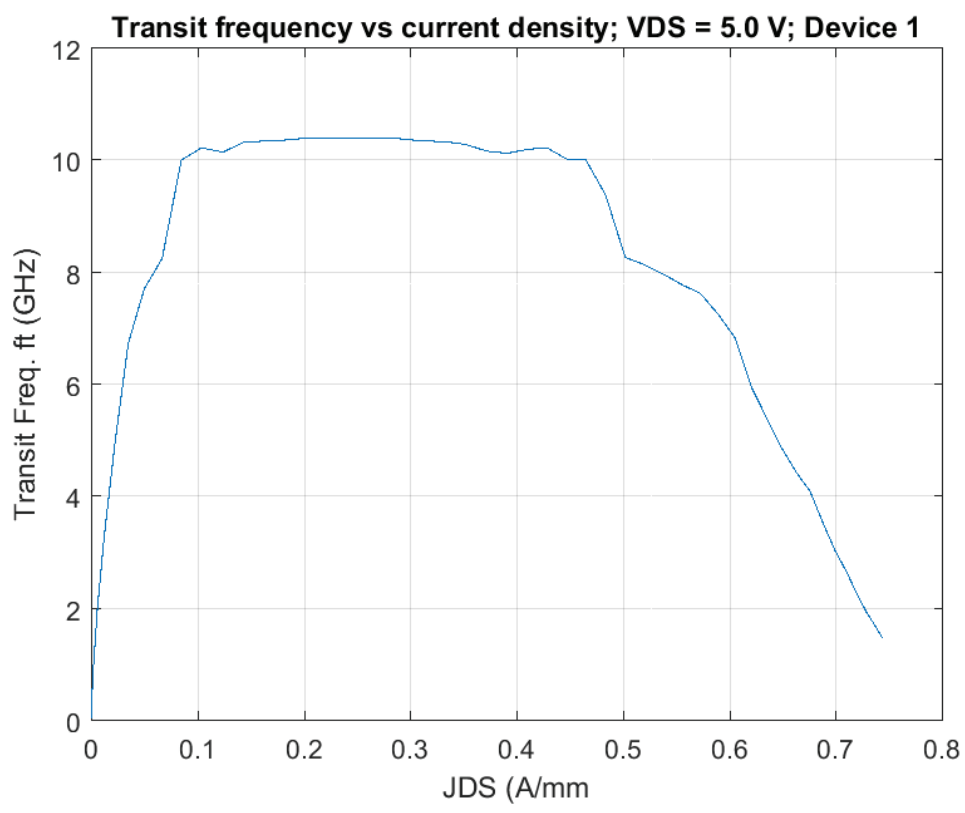


Figure A.2: Ft vs IDS, device $1, \operatorname{VDS}=9 \mathrm{~V}$

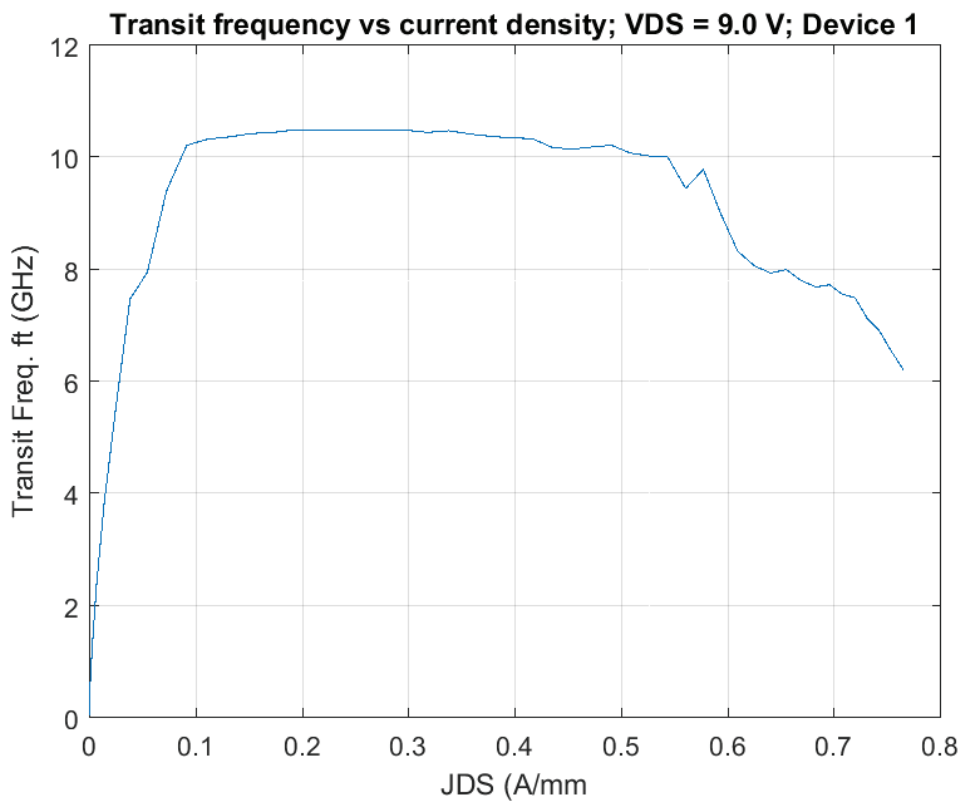

Figure A.3: Ft vs IDS, device $1, \operatorname{VDS}=9.5 \mathrm{~V}$

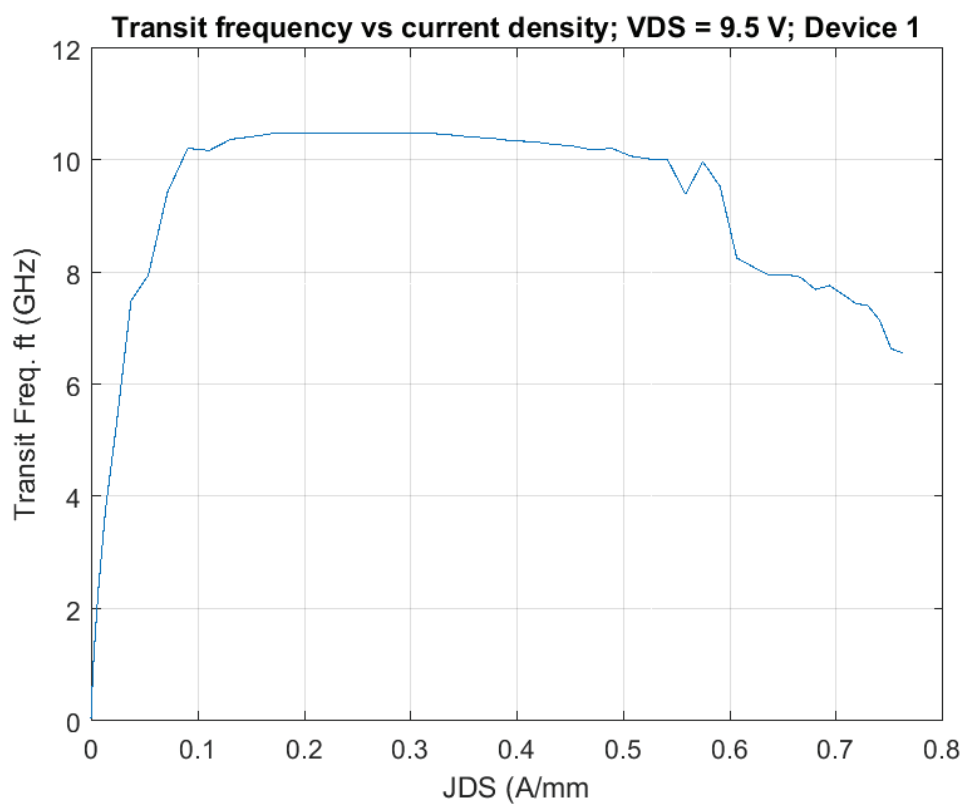


Figure A.4: Ft vs IDS, device $1, \operatorname{VDS}=10 \mathrm{~V}$

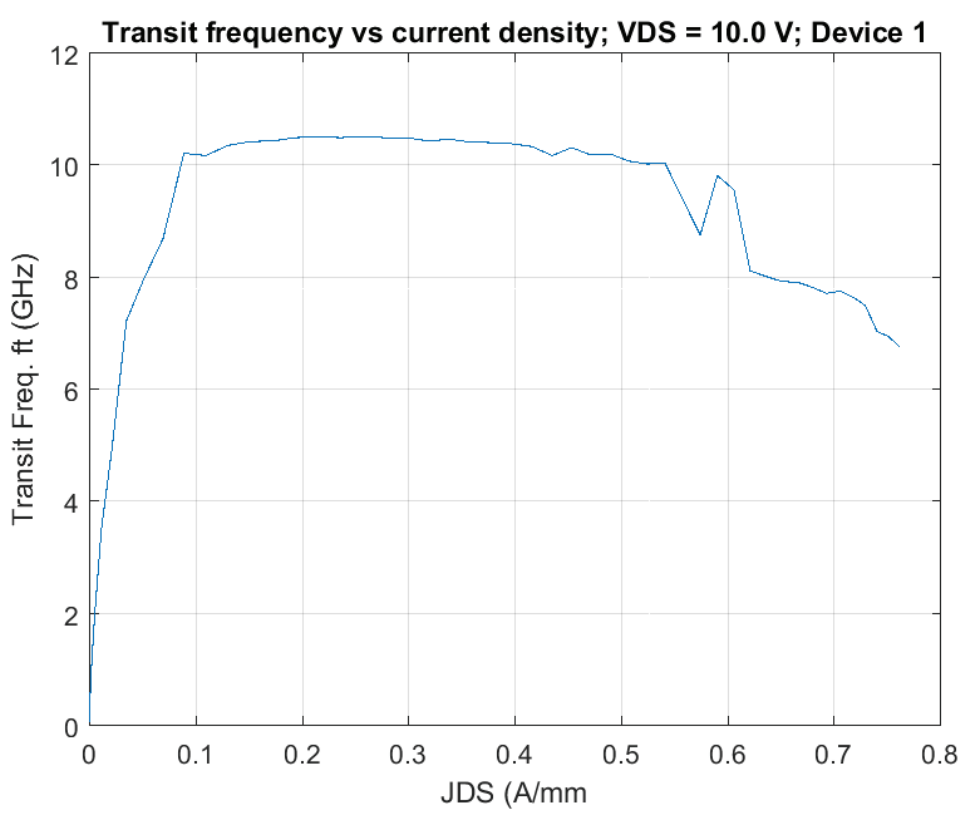

Figure A.5: Ft vs IDS, device 1, VDS $=10.5 \mathrm{~V}$

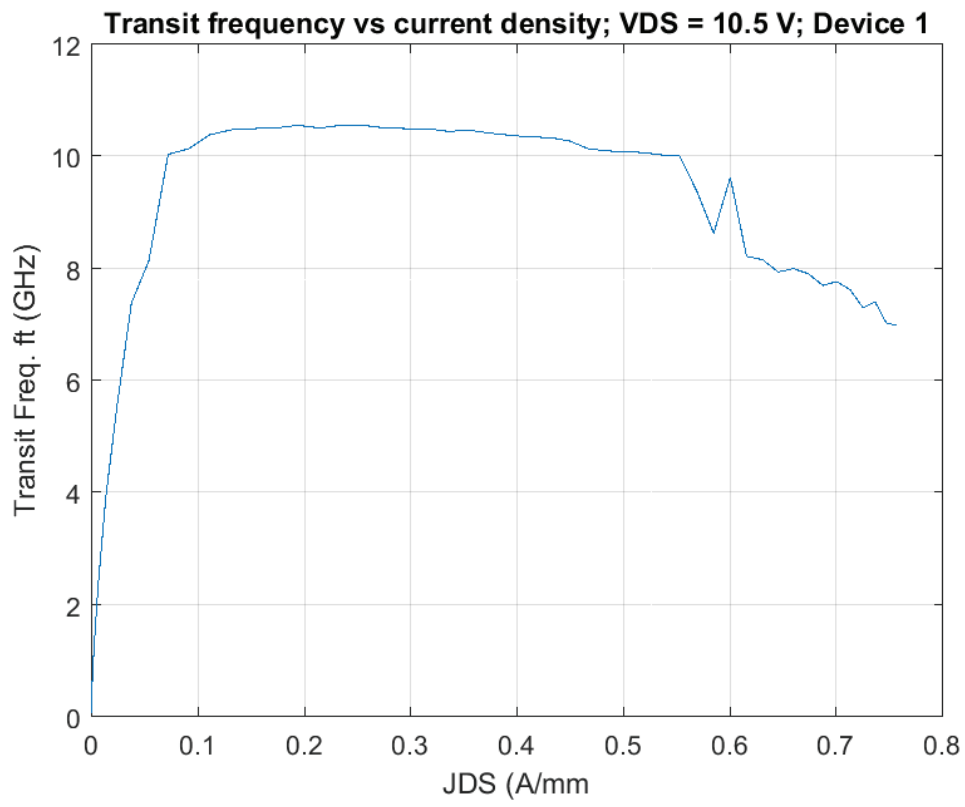


Figure A.6: Ft vs IDS, device $1, \mathrm{VDS}=11 \mathrm{~V}$

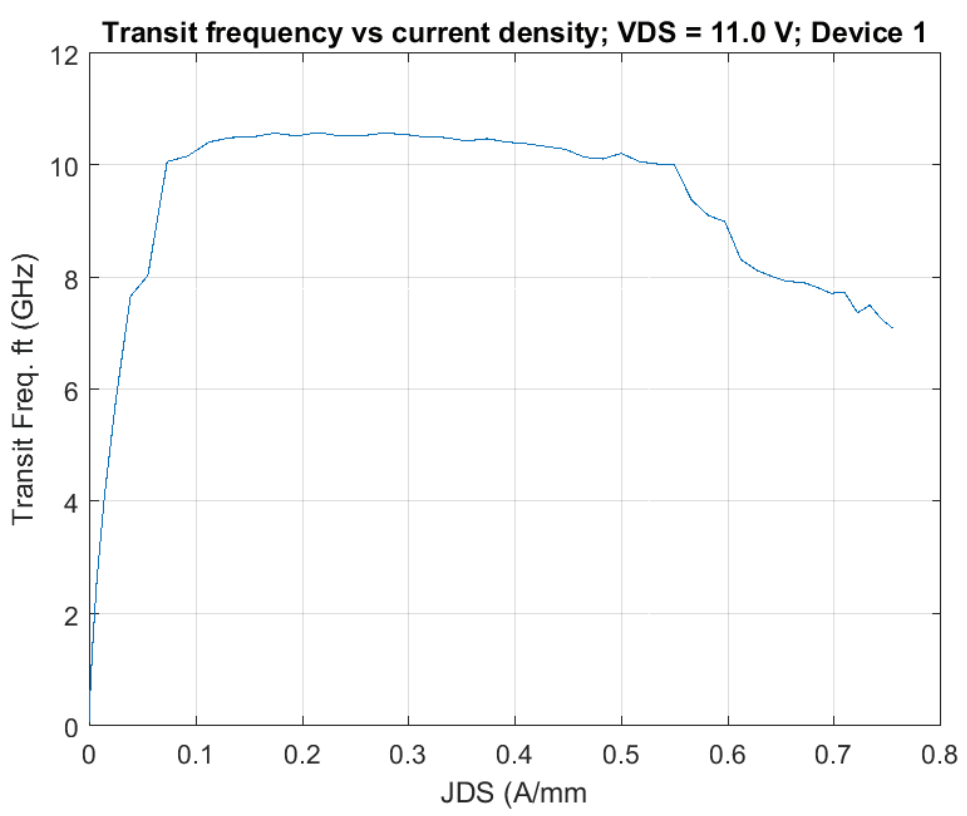

FiguRE A.7: Ft vs IDS, device 1, VDS $=15 \mathrm{~V}$

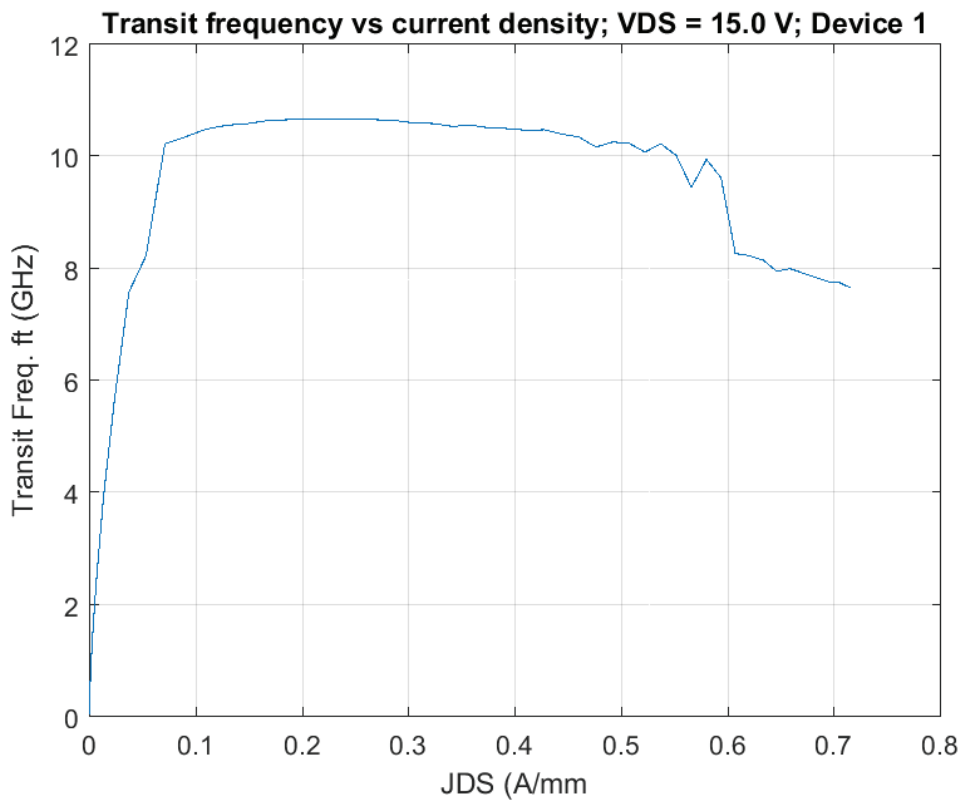


Figure A.8: Ft vs IDS, device 1, VDS $=20 \mathrm{~V}$

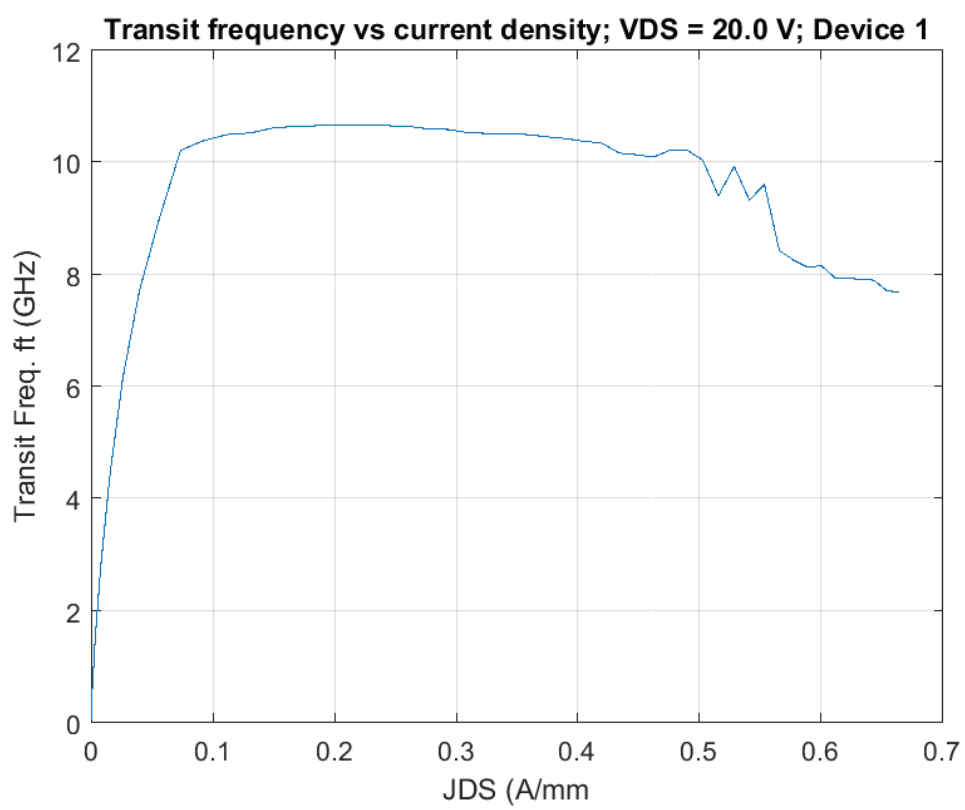




\section{A.2 Device 2}

Figure A.9: Ft vs IDS, device 2, VDS $=5 \mathrm{~V}$

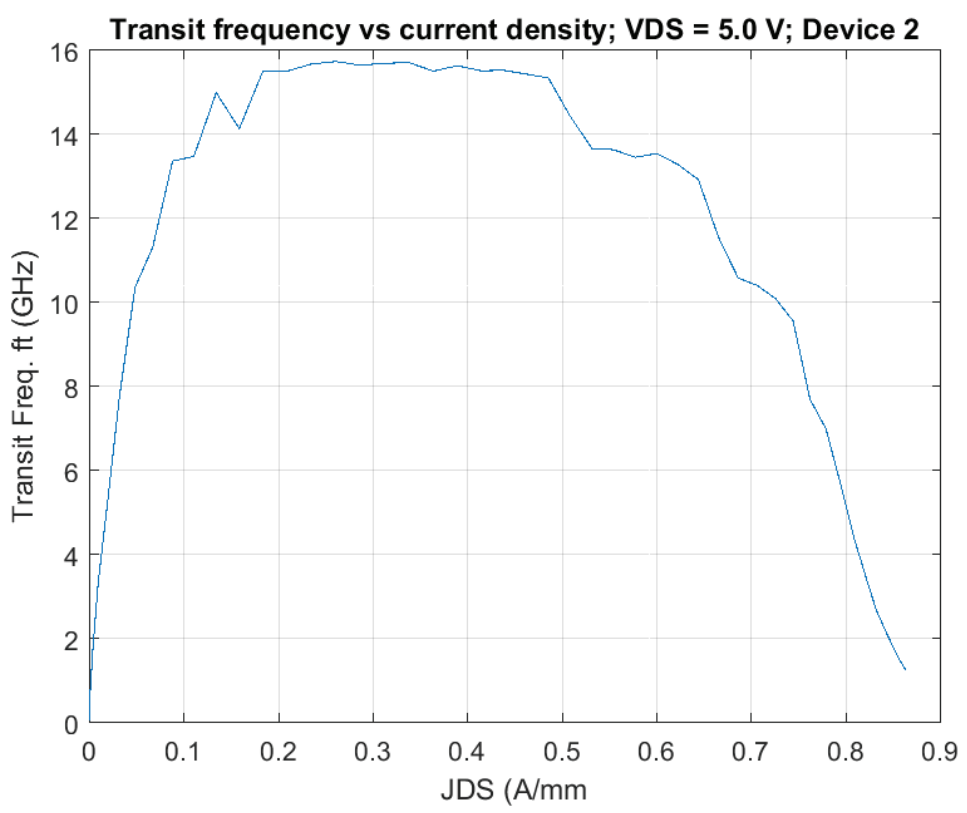


Figure A.10: Ft vs IDS, device 2, VDS $=9 \mathrm{~V}$

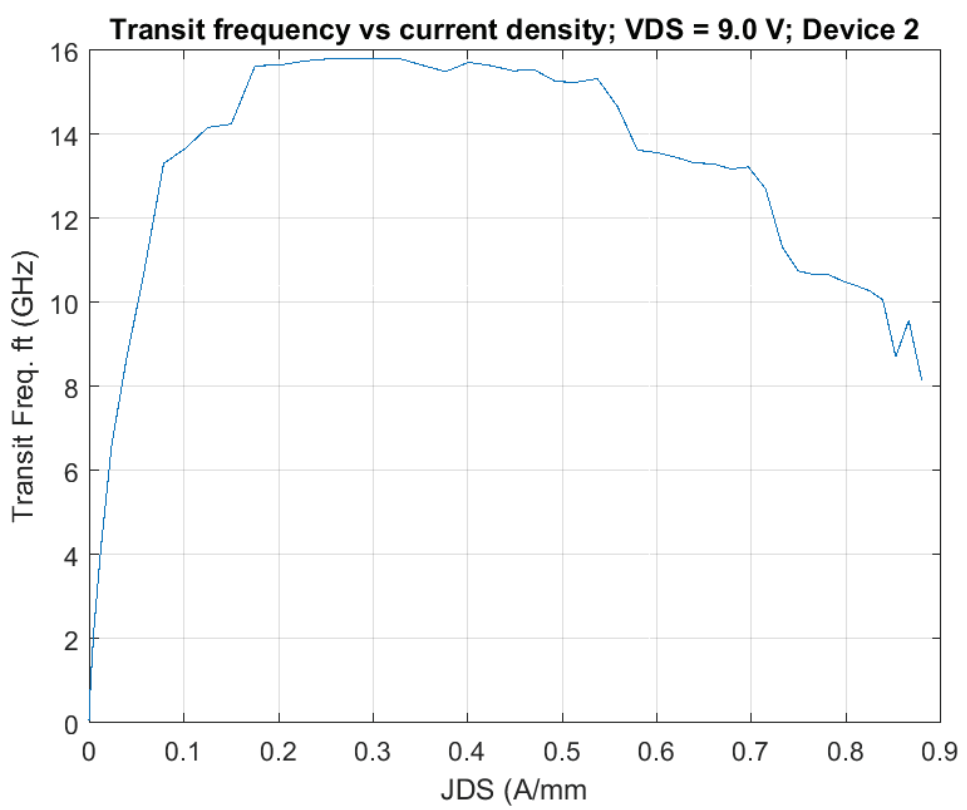

Figure A.11: Ft vs IDS, device $2, \operatorname{VDS}=9.5 \mathrm{~V}$

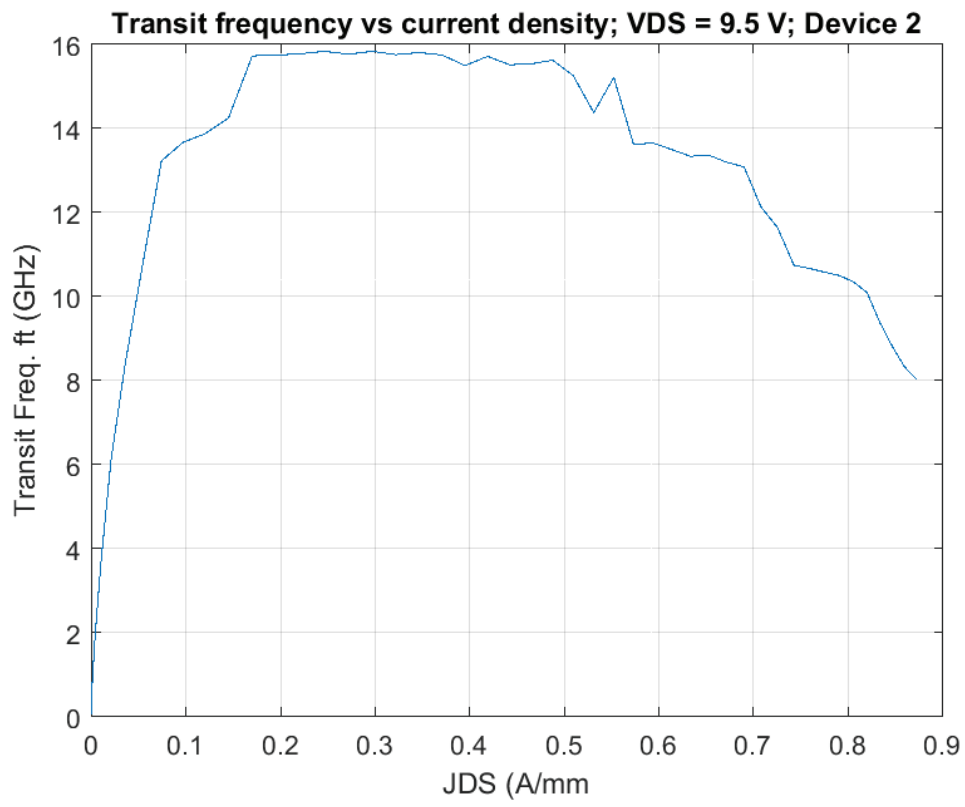


Figure A.12: Ft vs IDS, device 2, VDS $=10 \mathrm{~V}$

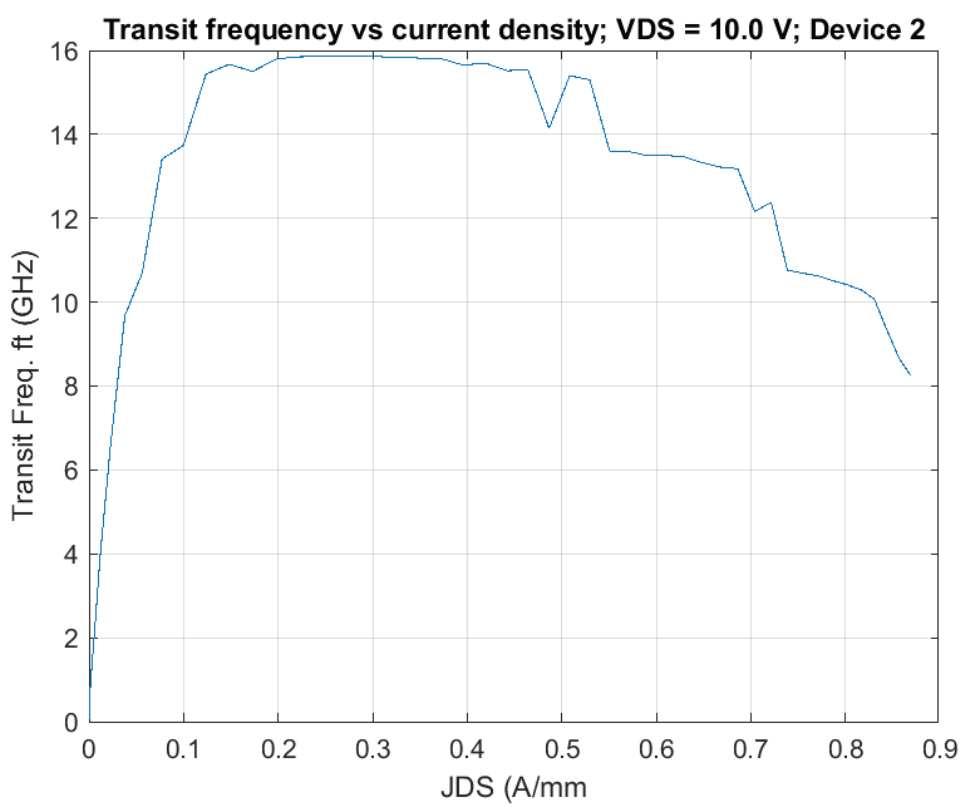

Figure A.13: Ft vs IDS, device 2, VDS $=10.5 \mathrm{~V}$

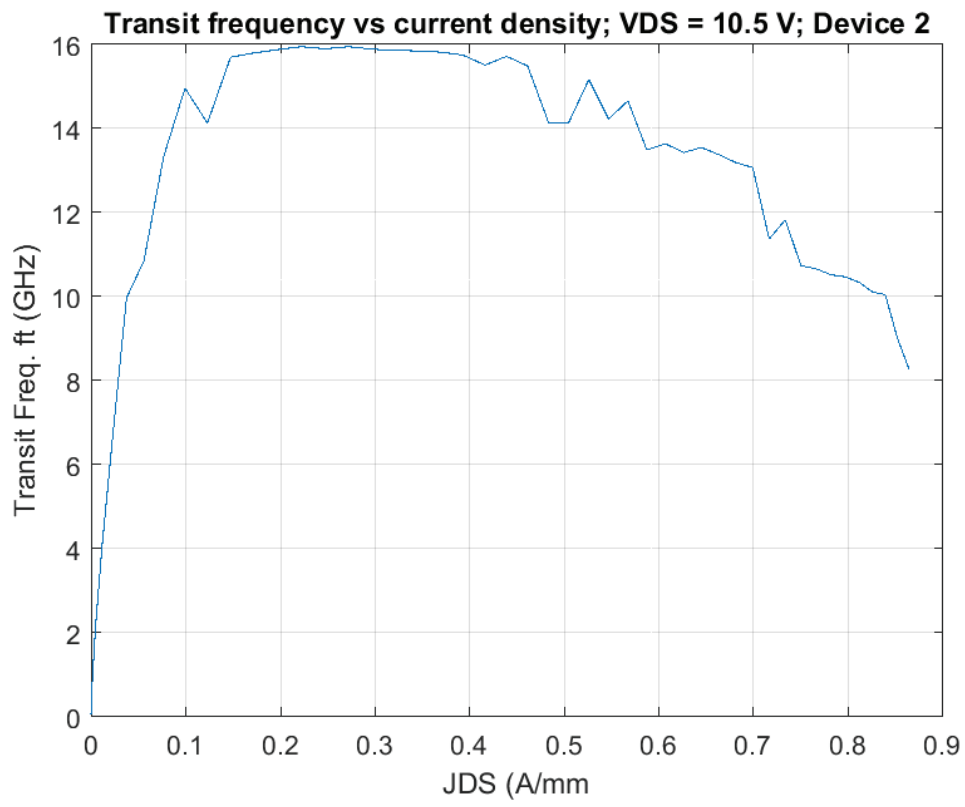


Figure A.14: Ft vs IDS, device 2, VDS $=11 \mathrm{~V}$

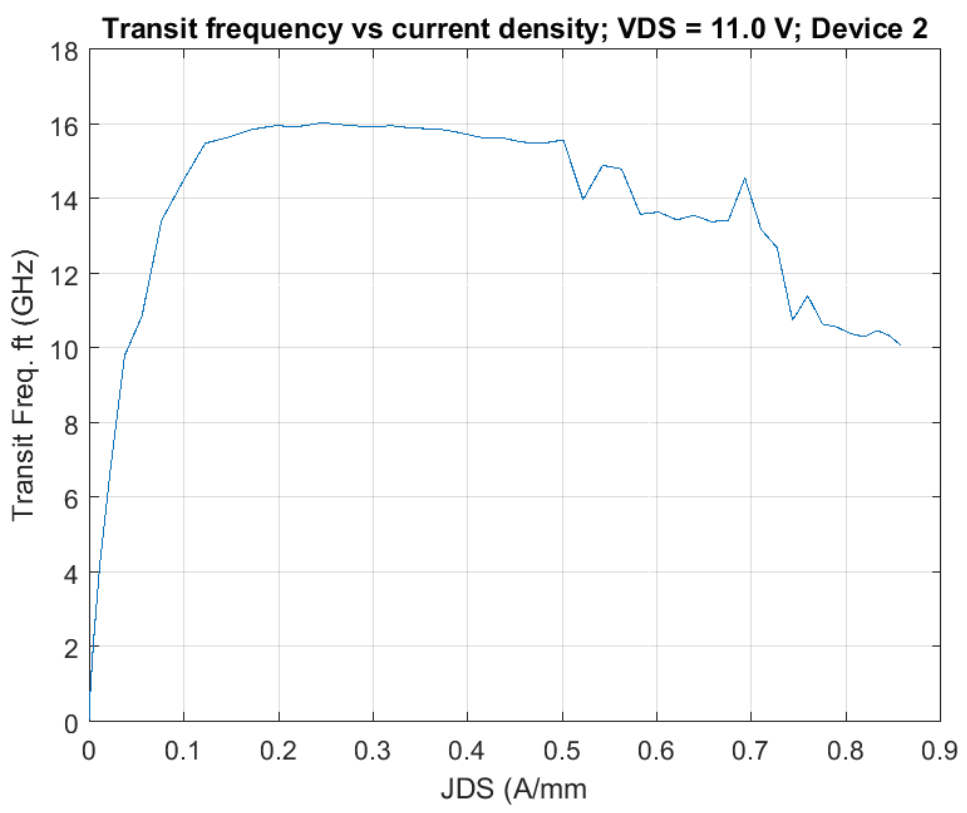

Figure A.15: Ft vs IDS, device 2, VDS $=15 \mathrm{~V}$

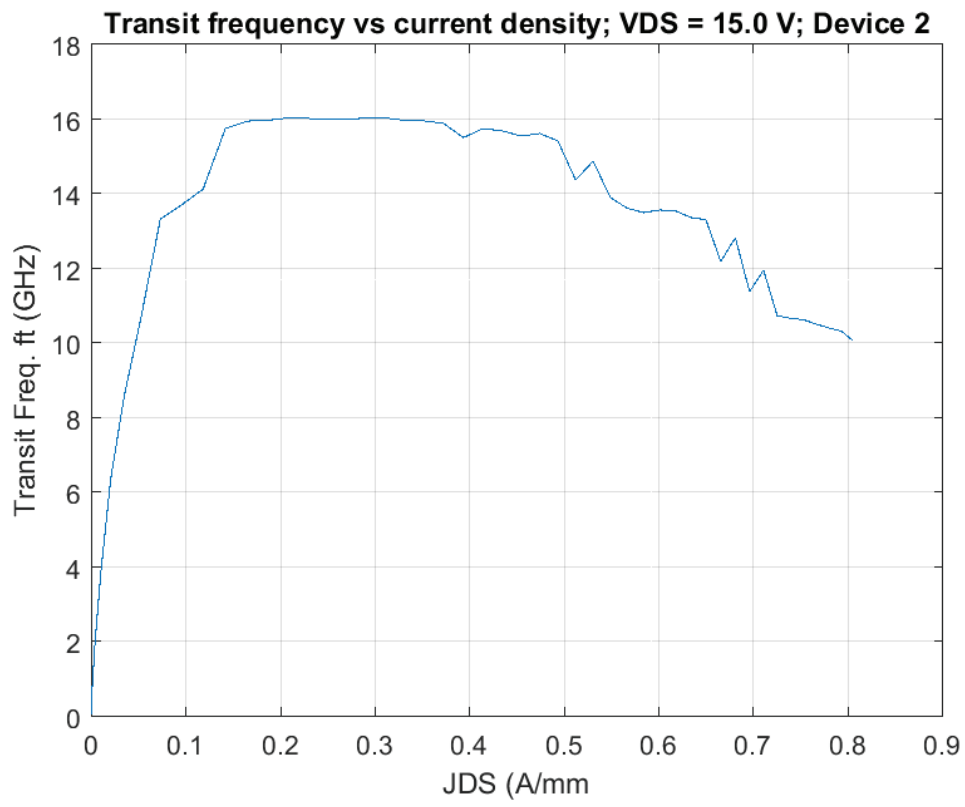


Figure A.16: Ft vs IDS, device 2, VDS $=20 \mathrm{~V}$

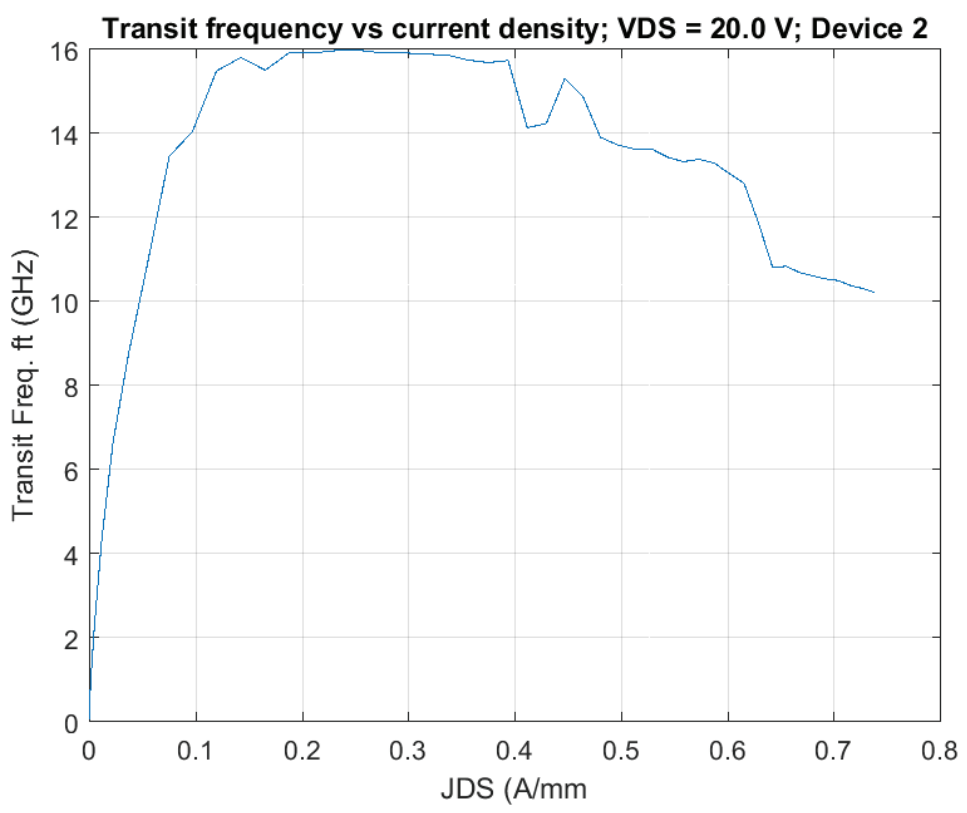




\section{Appendix B}

labelB This appendix contains all matlab code used to capture and characterize active inductor, BPF, and HFETs

\section{B.1 sweepDAC.m}

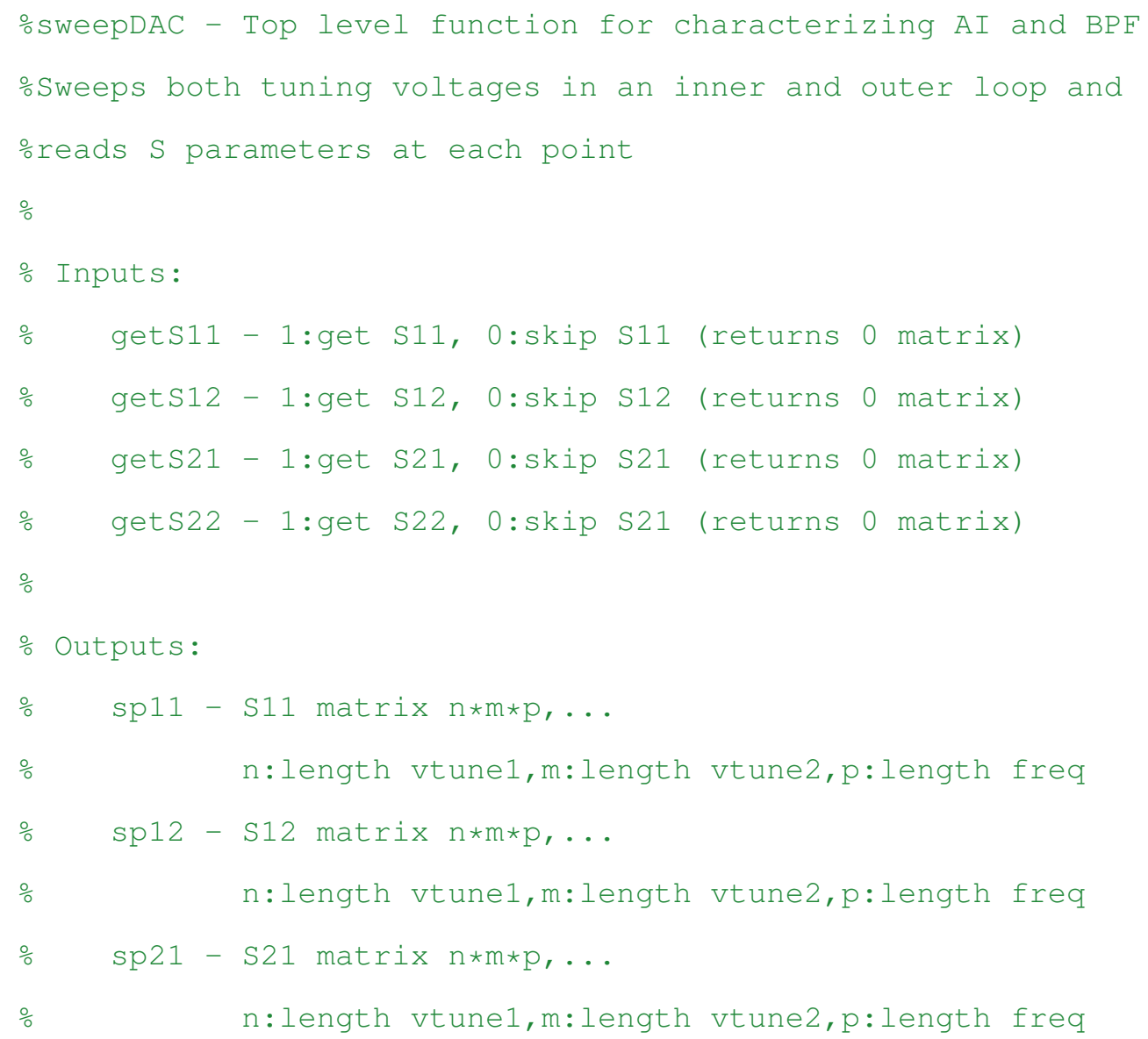




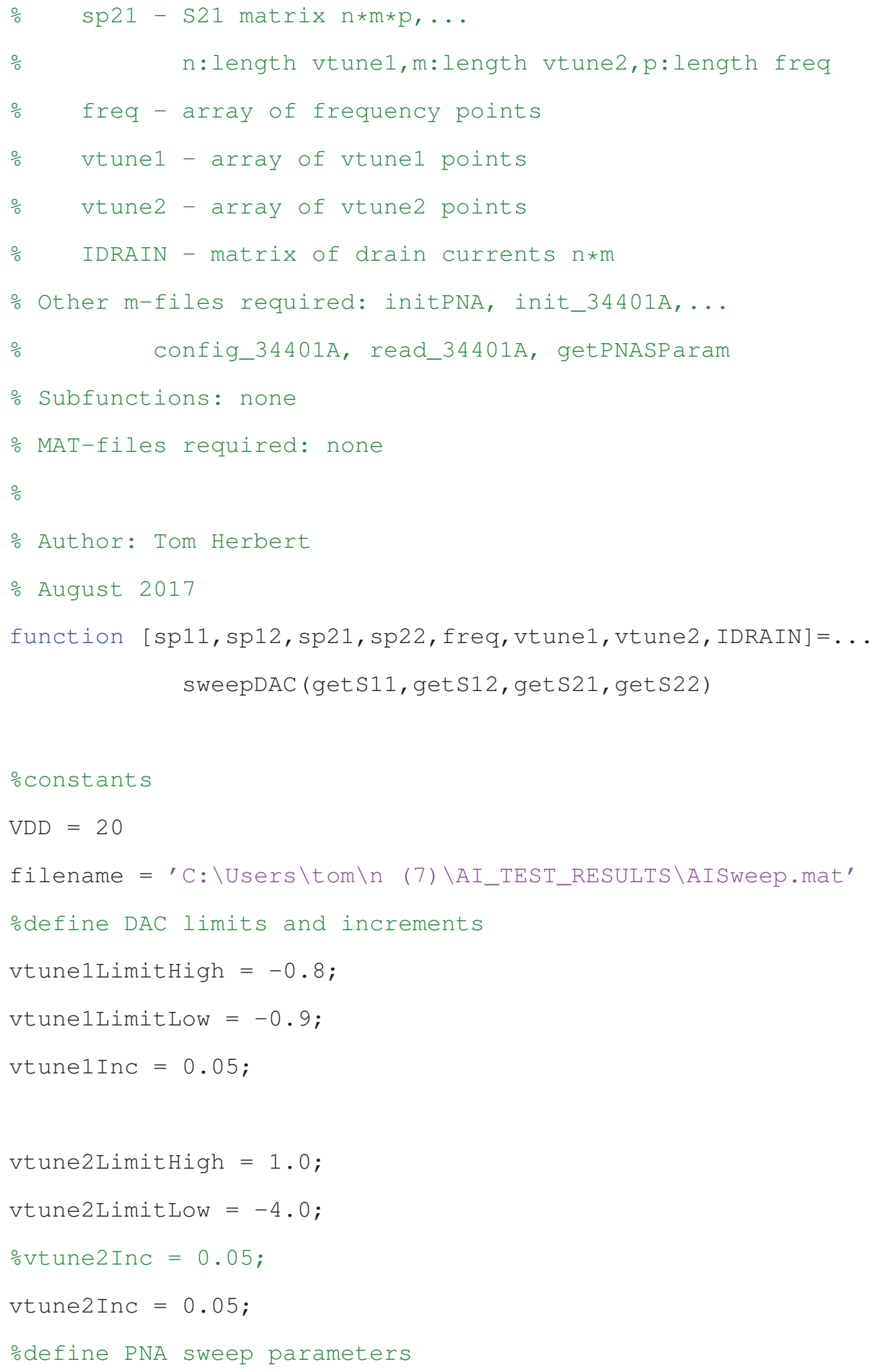




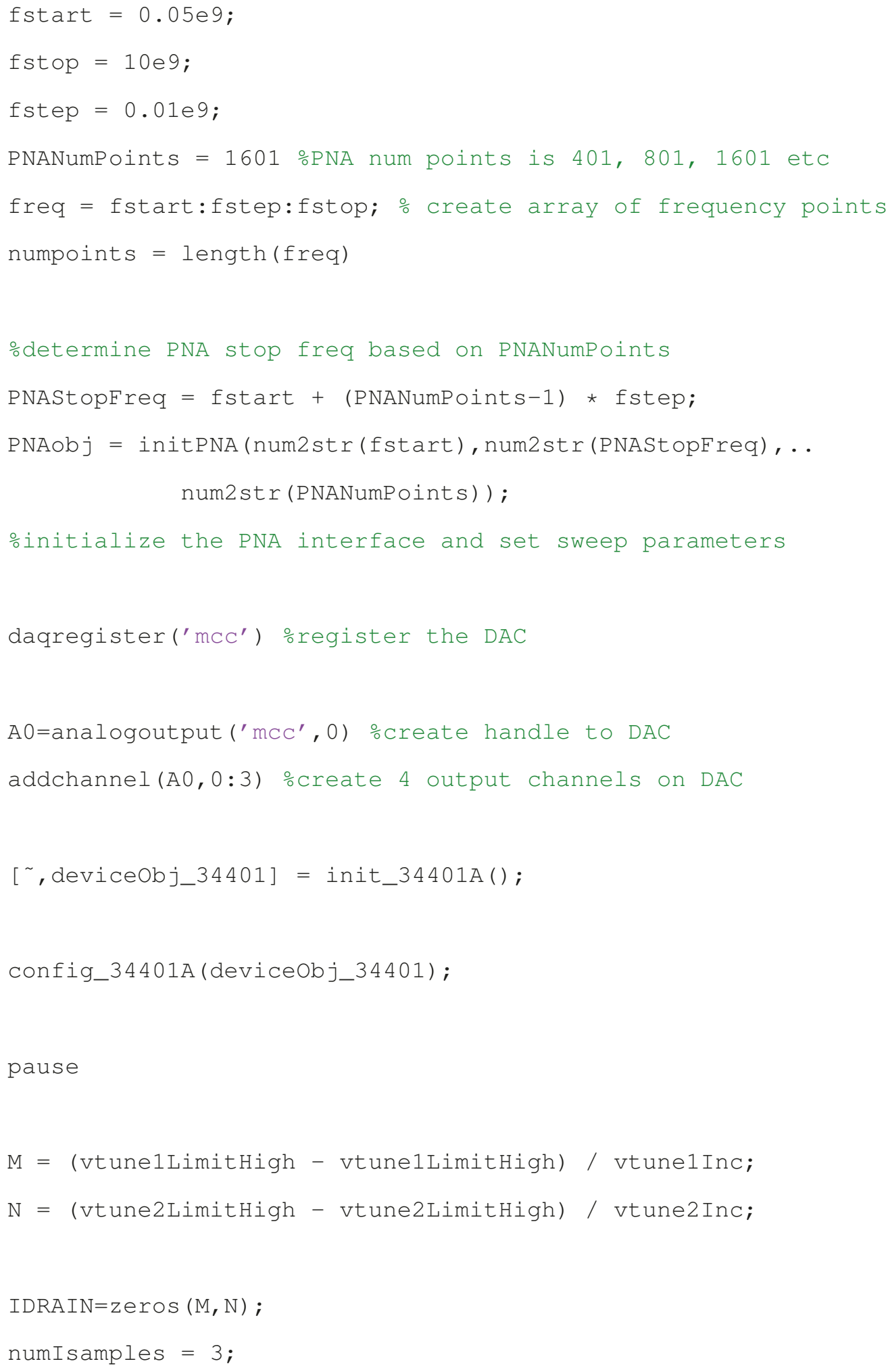




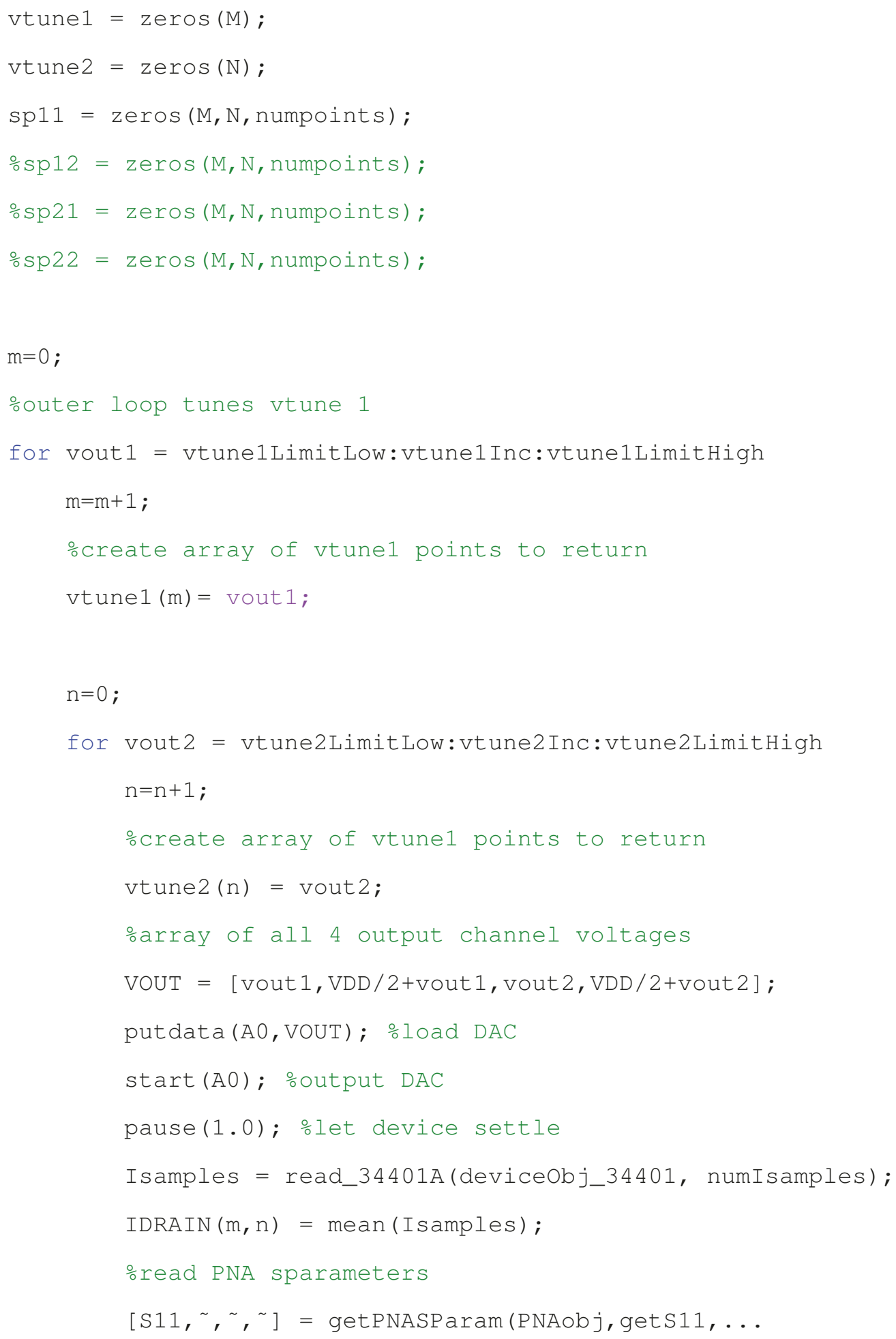




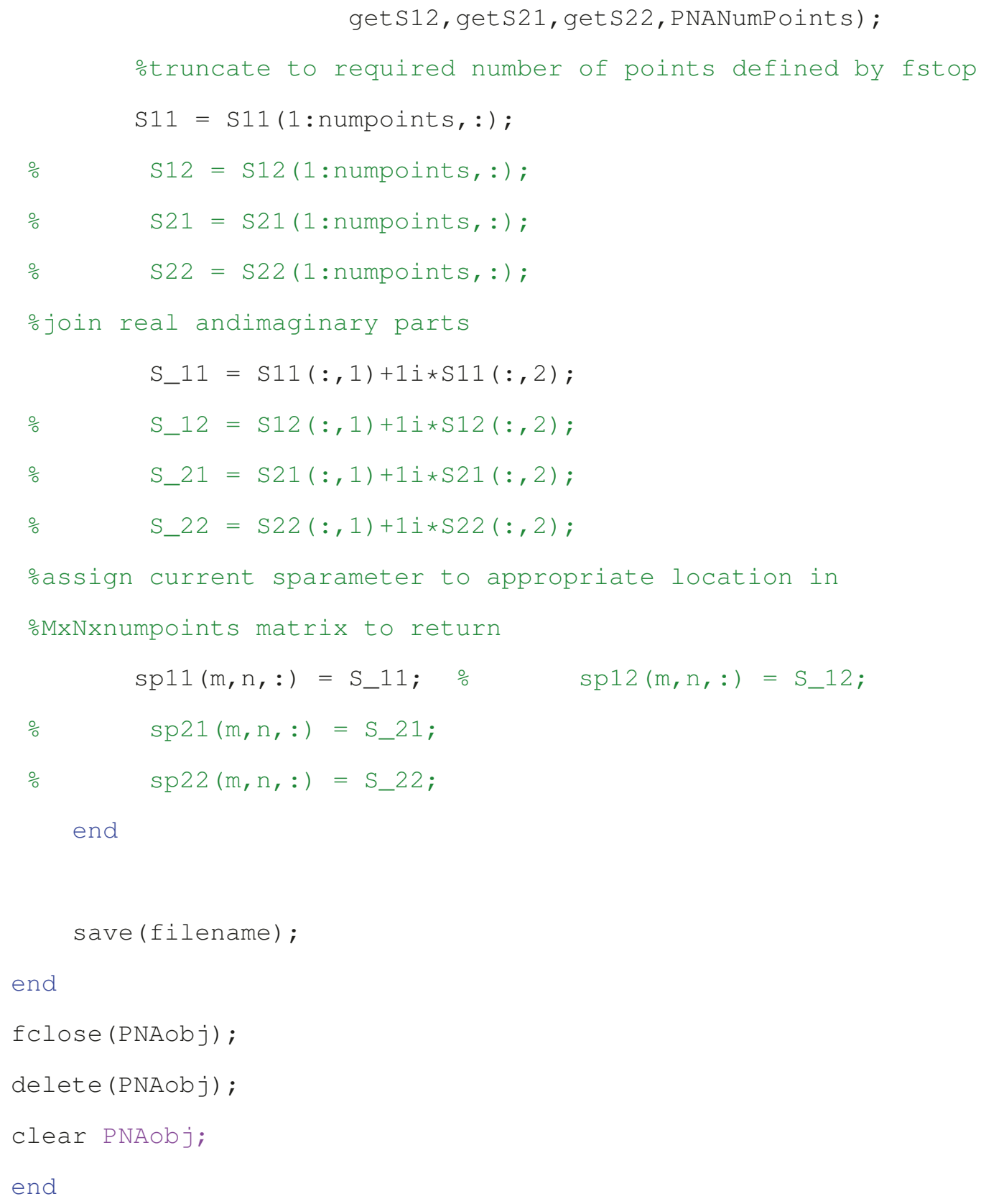




\section{B.2 GAN_IDvsVDS.m}

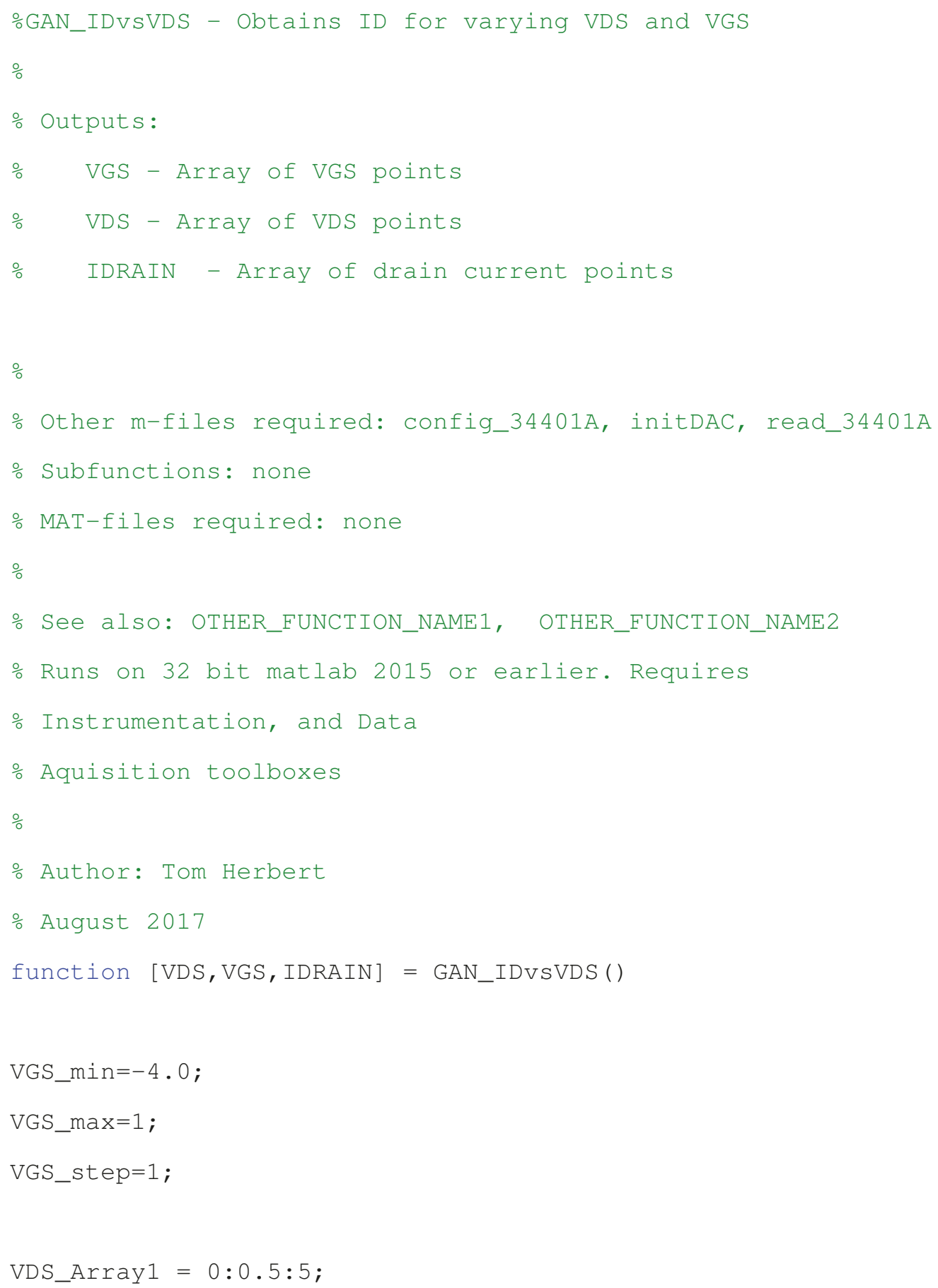




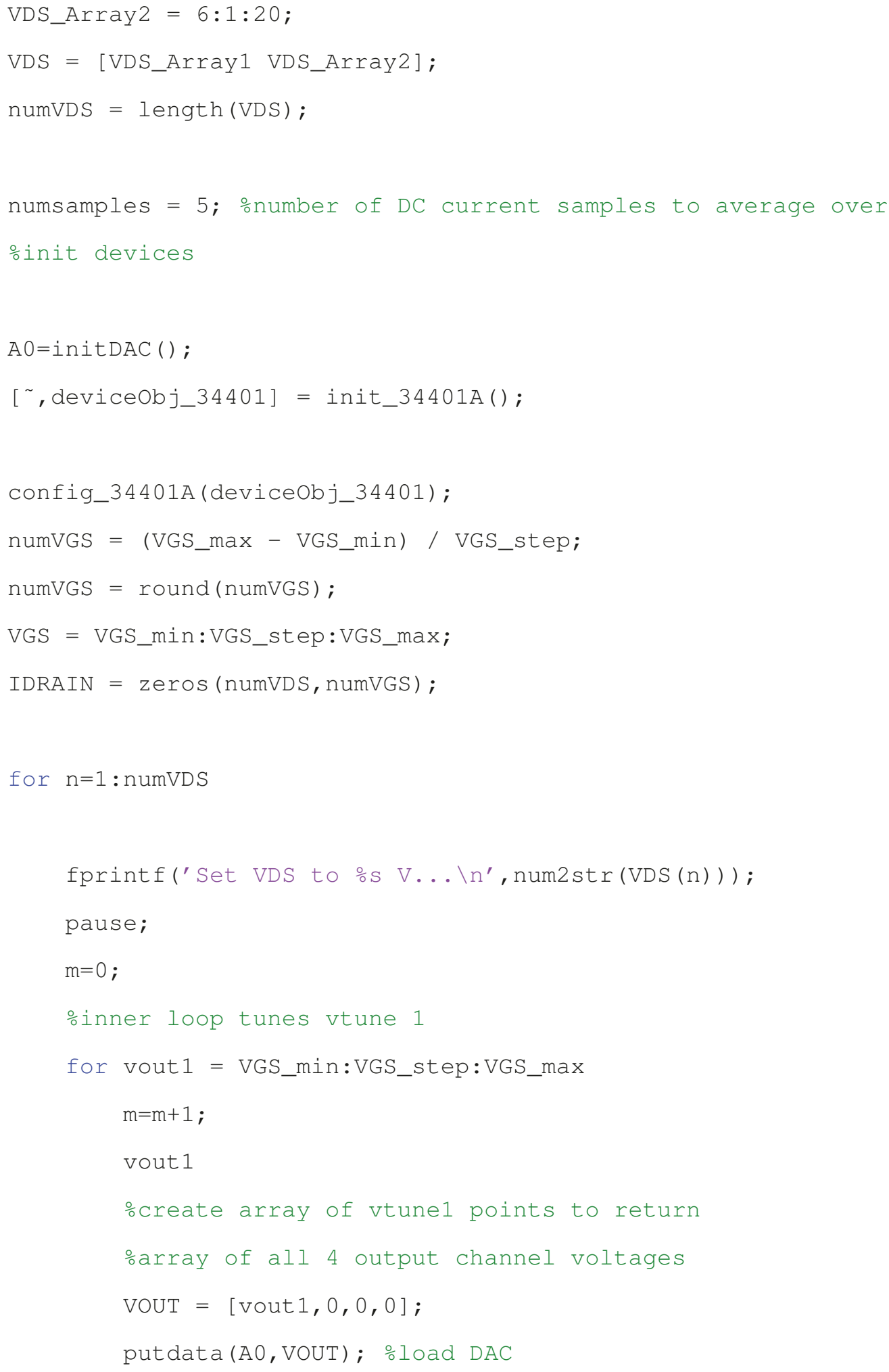




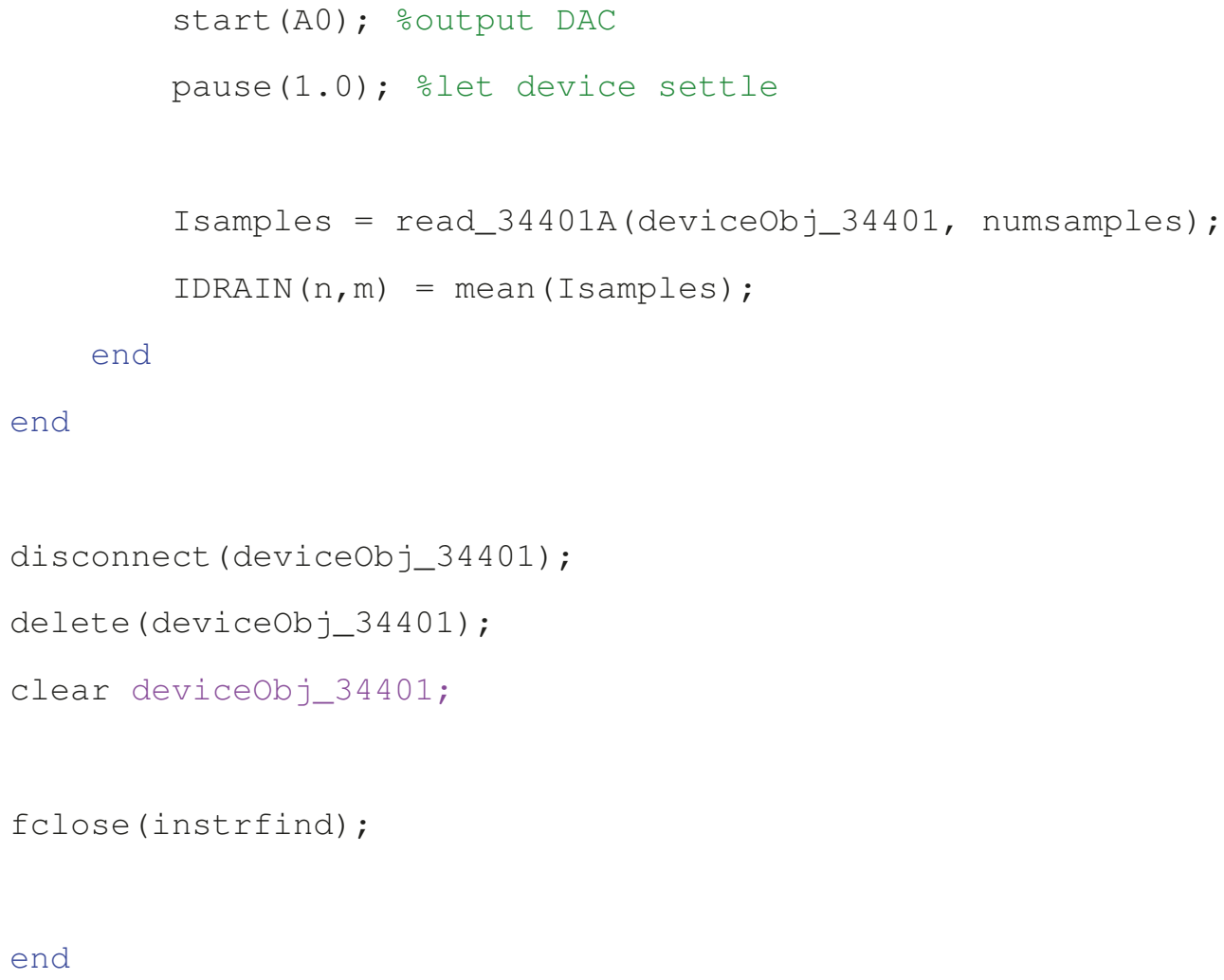

\section{B.3 GAN_CZ.m}

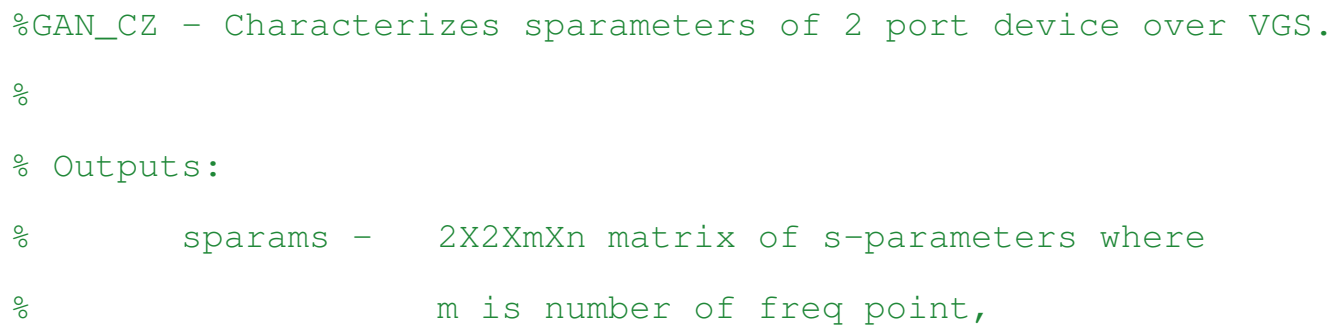




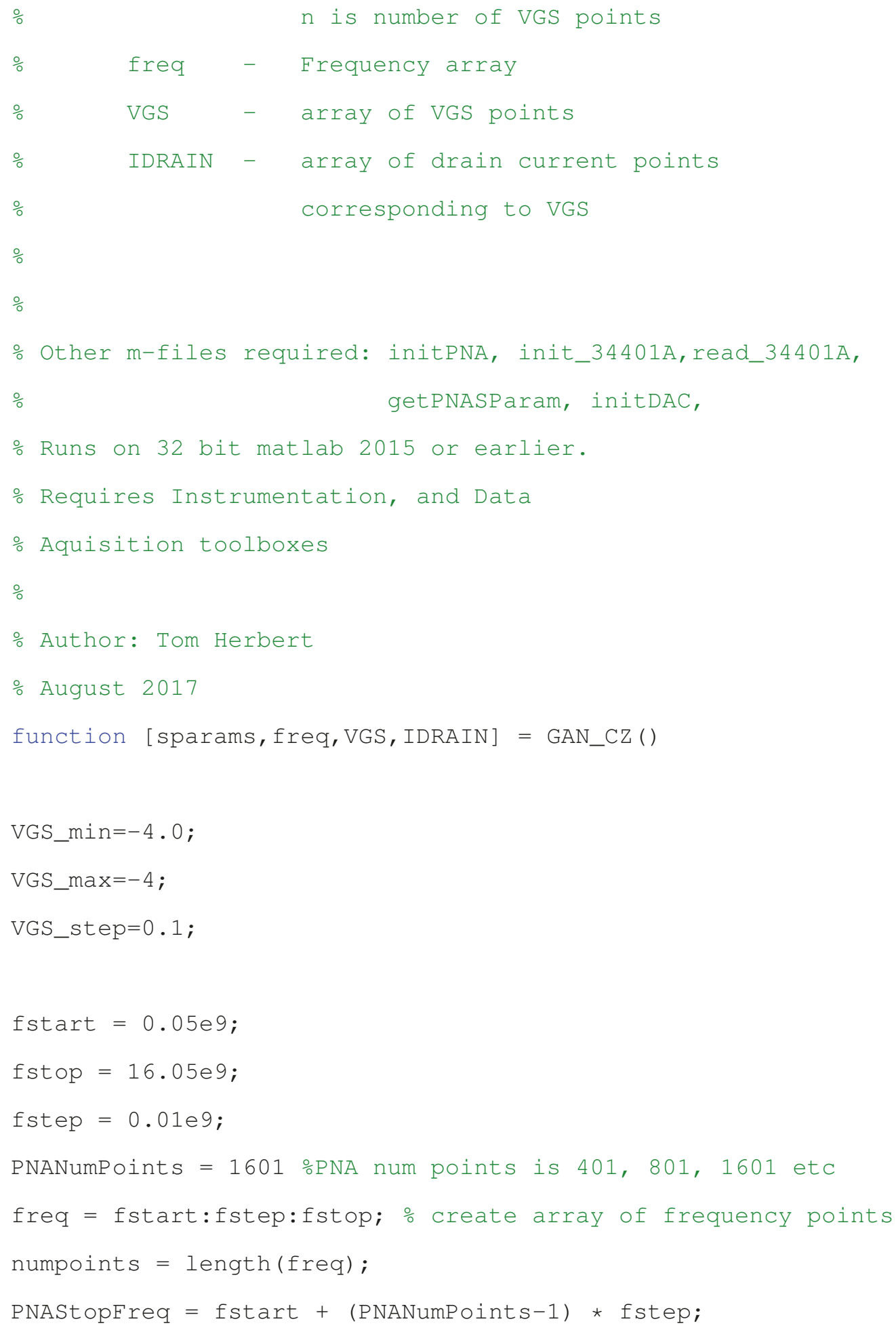




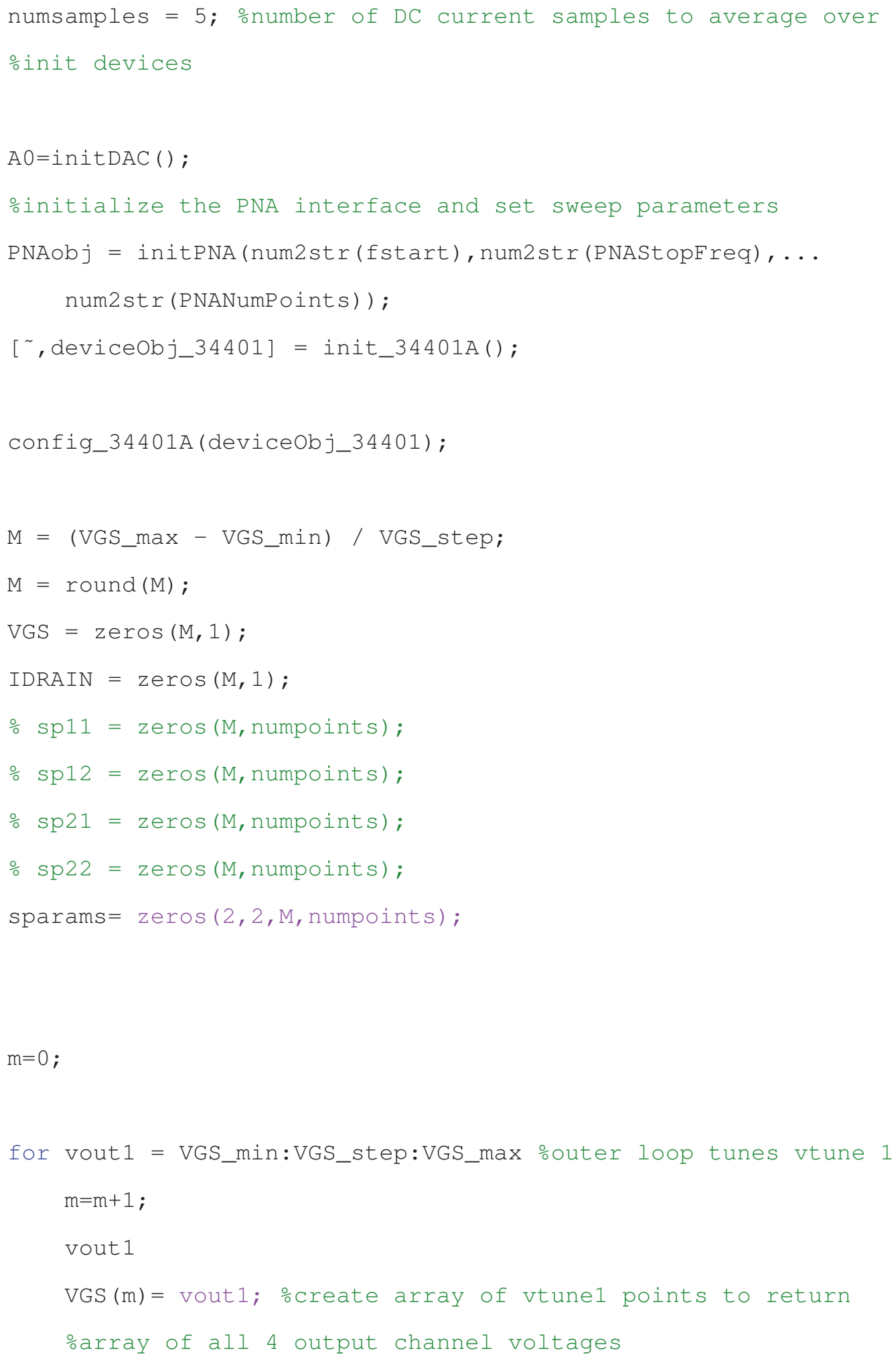




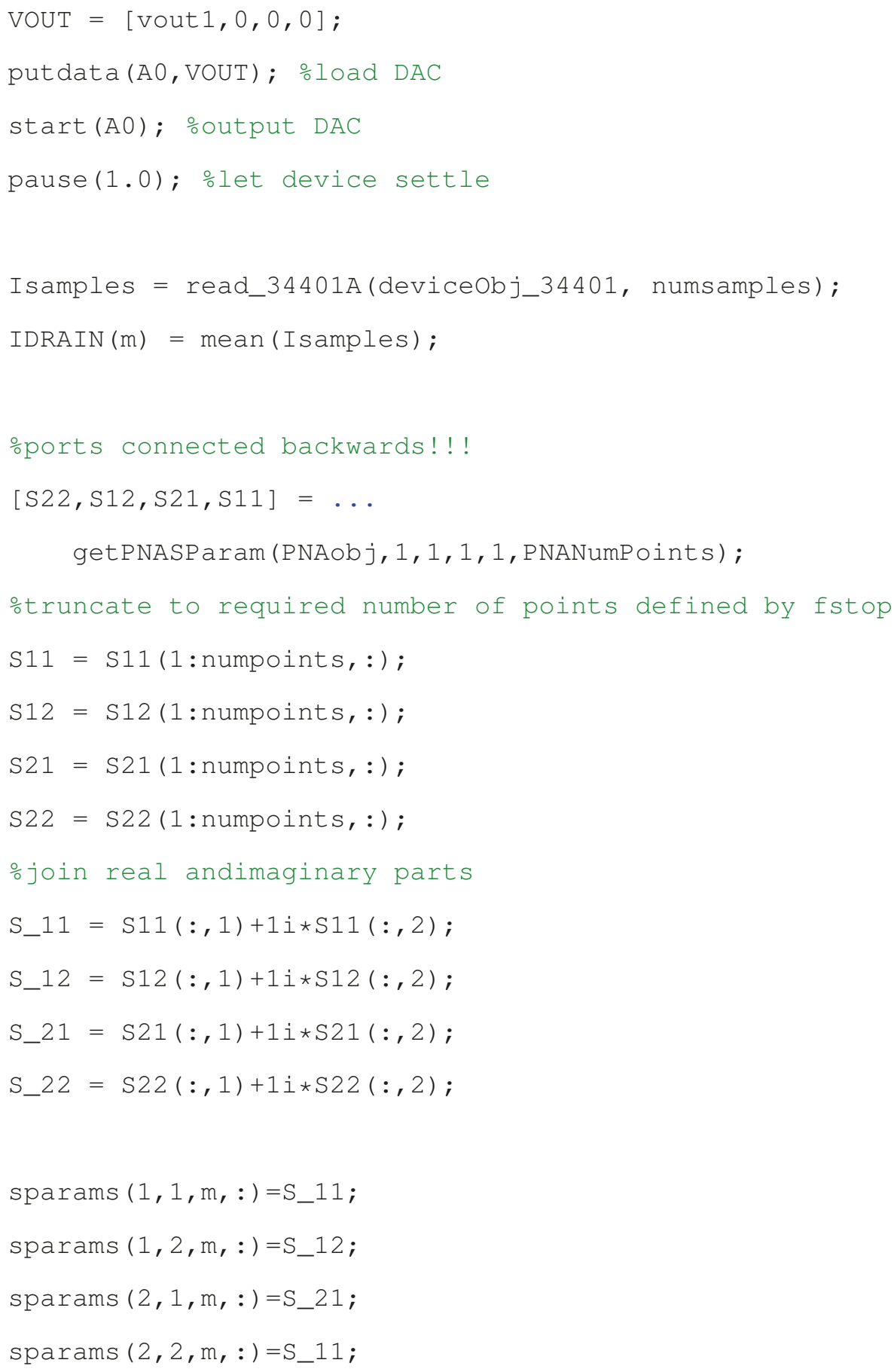




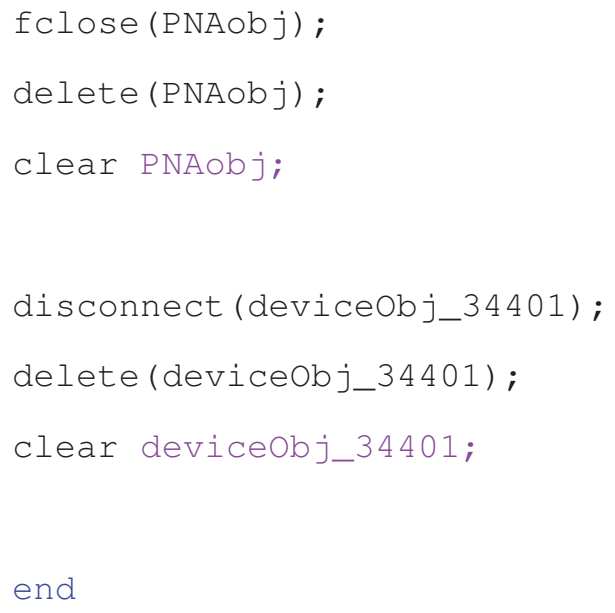

\section{B.4 getPNASparam.m}

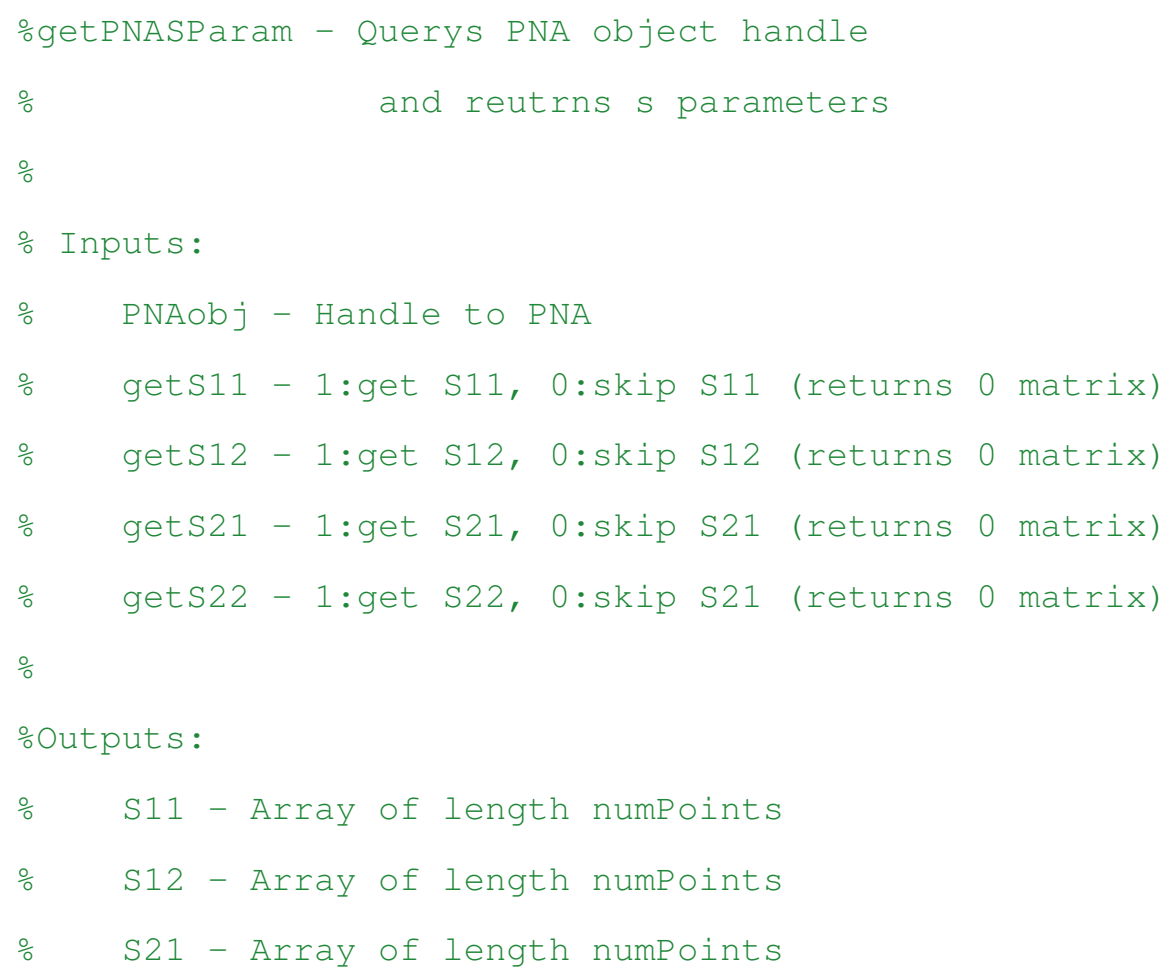




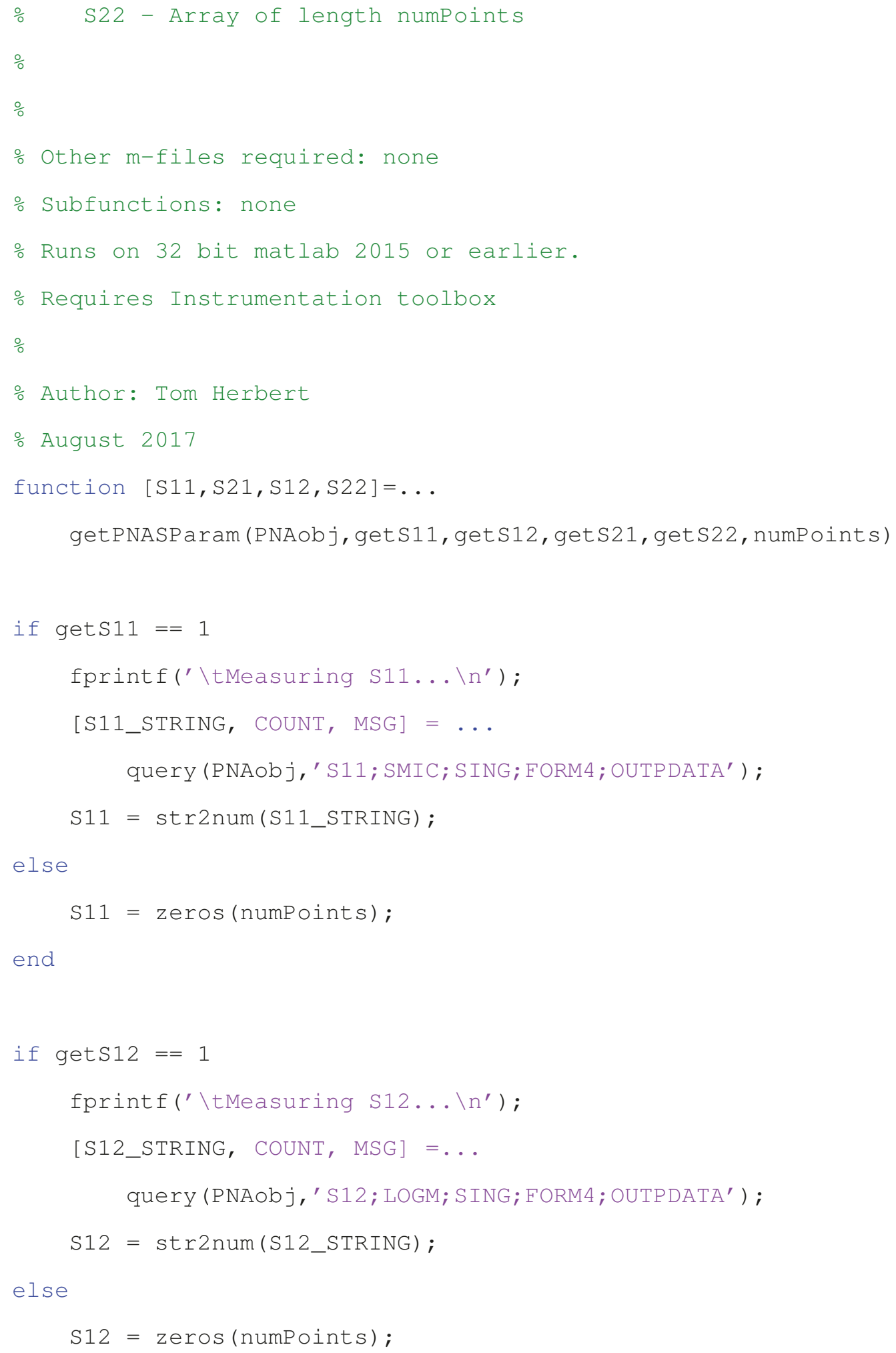




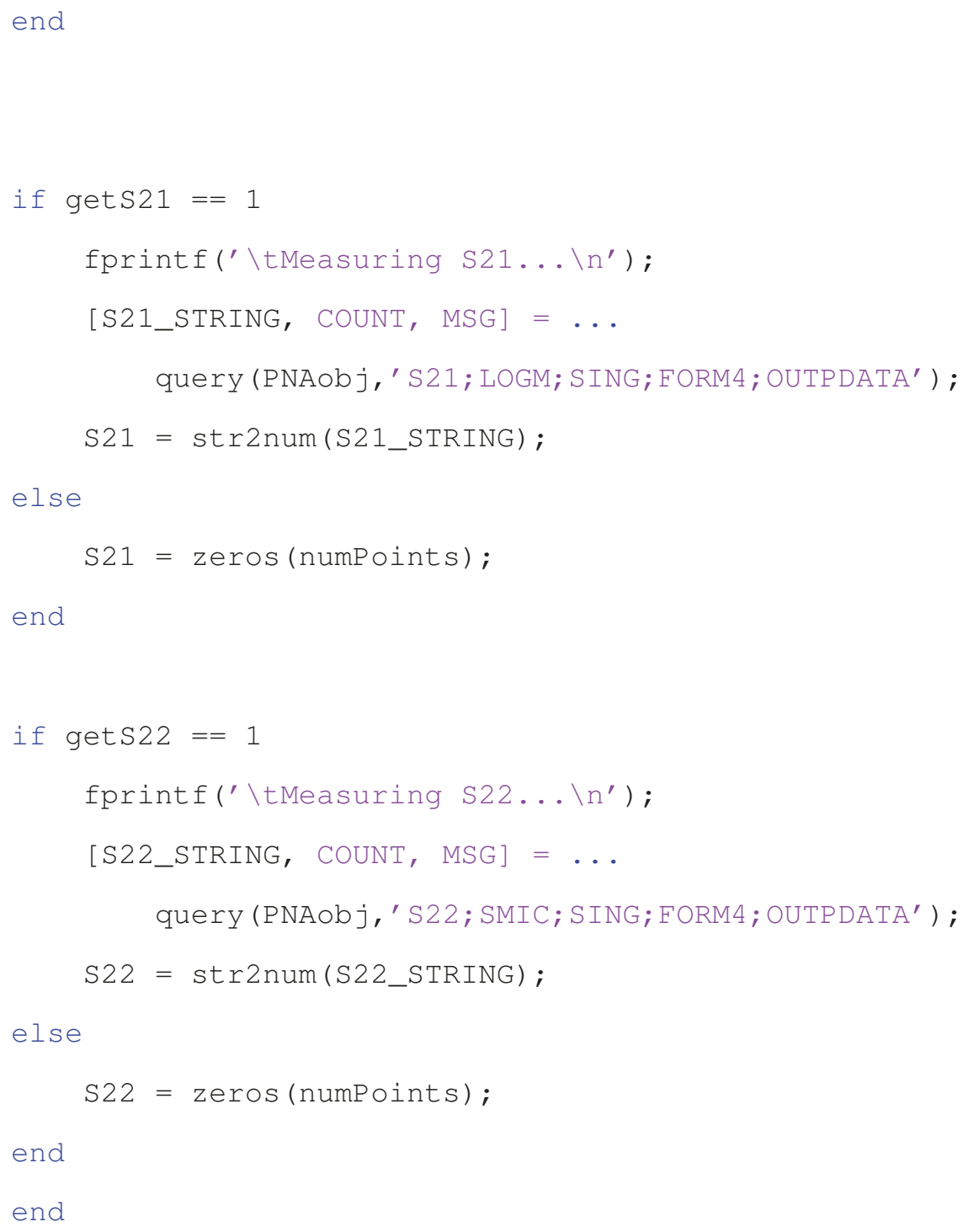

\section{B.5 initPNA.m}

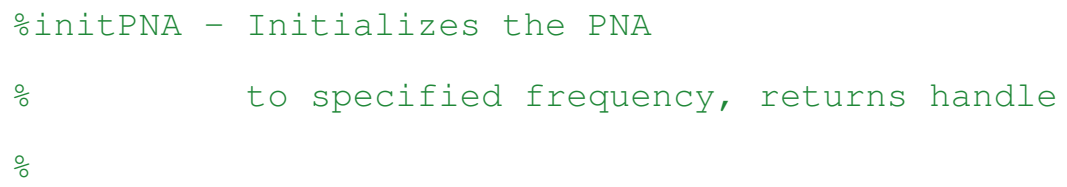




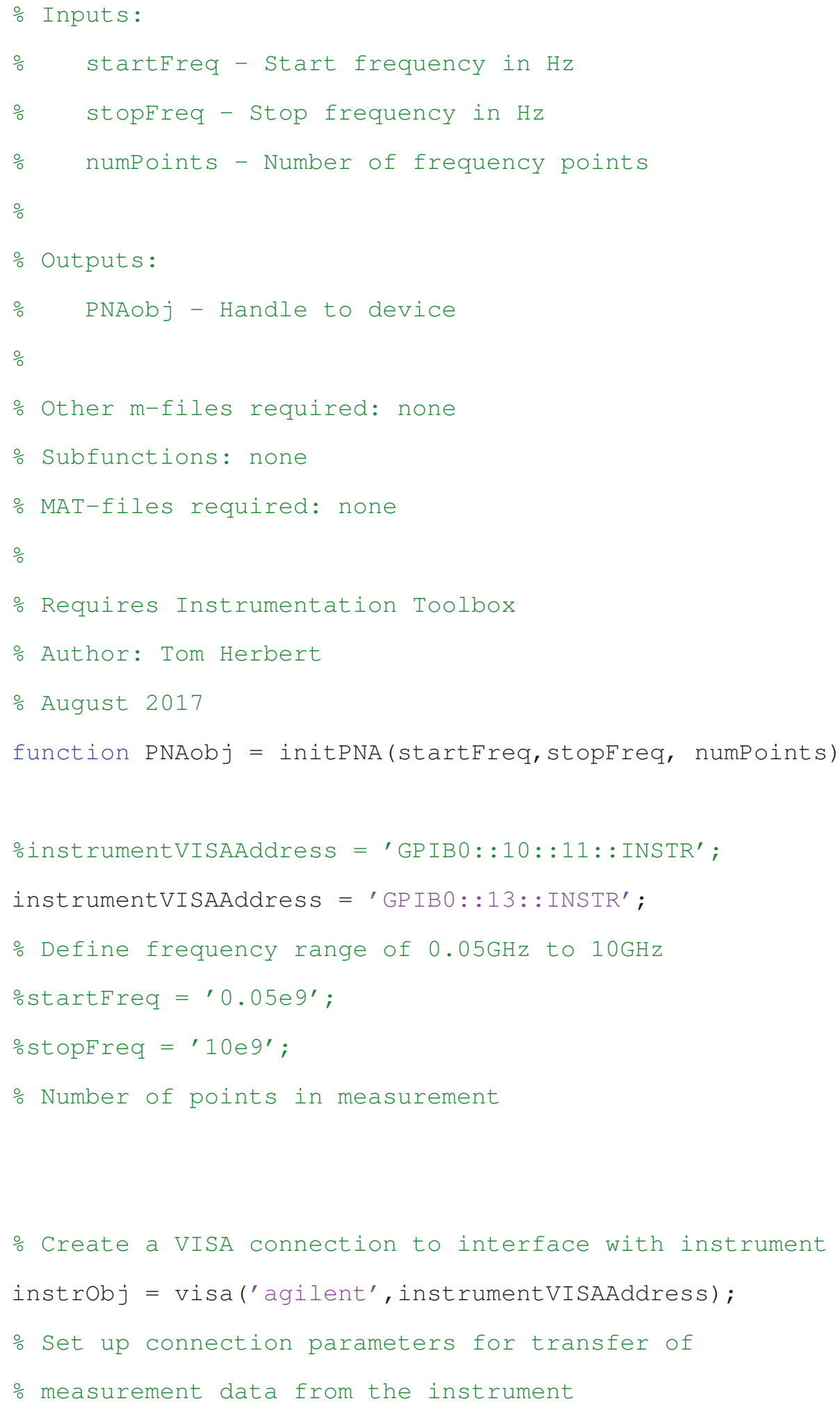




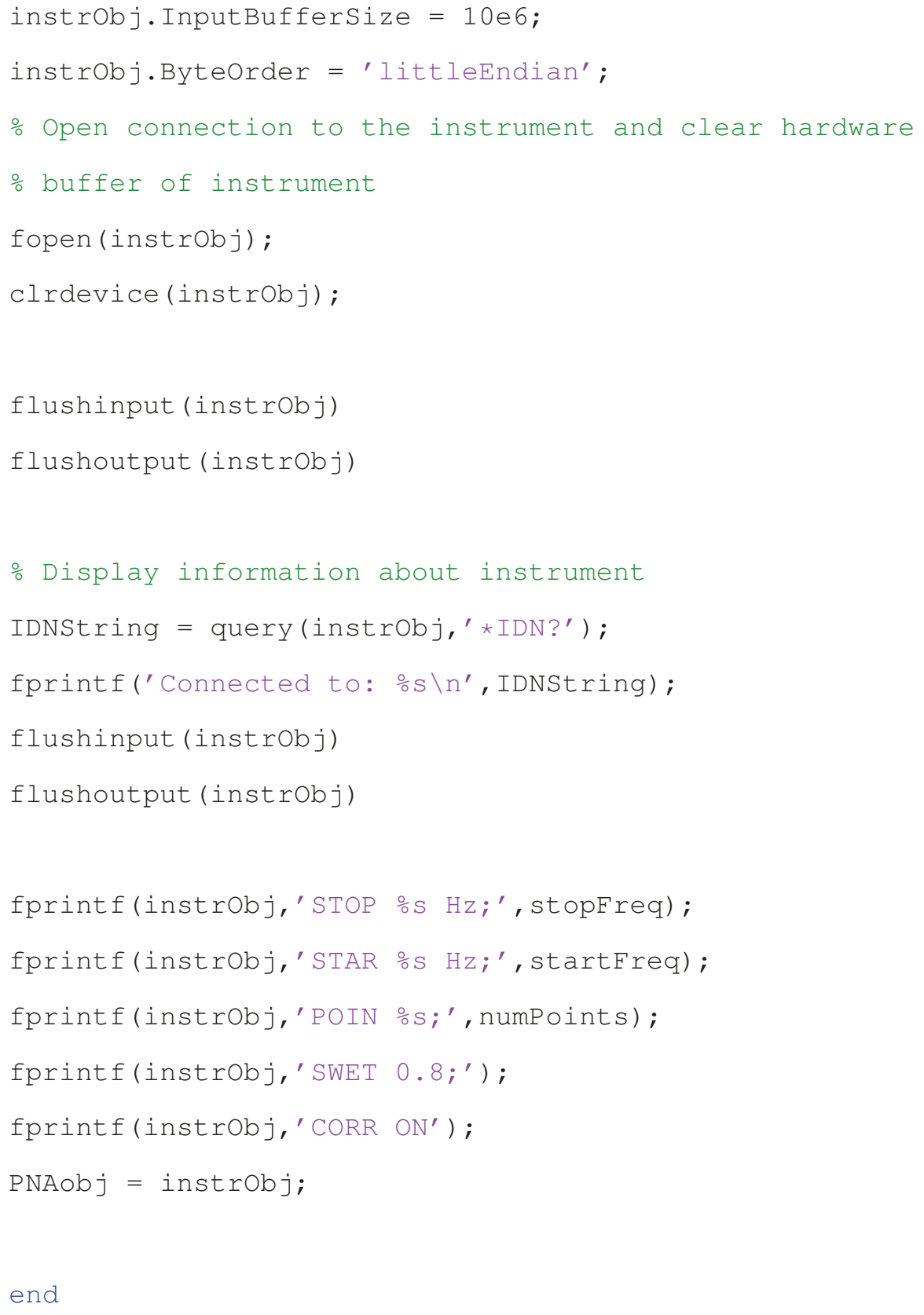




\section{B.6 initDAC.m}

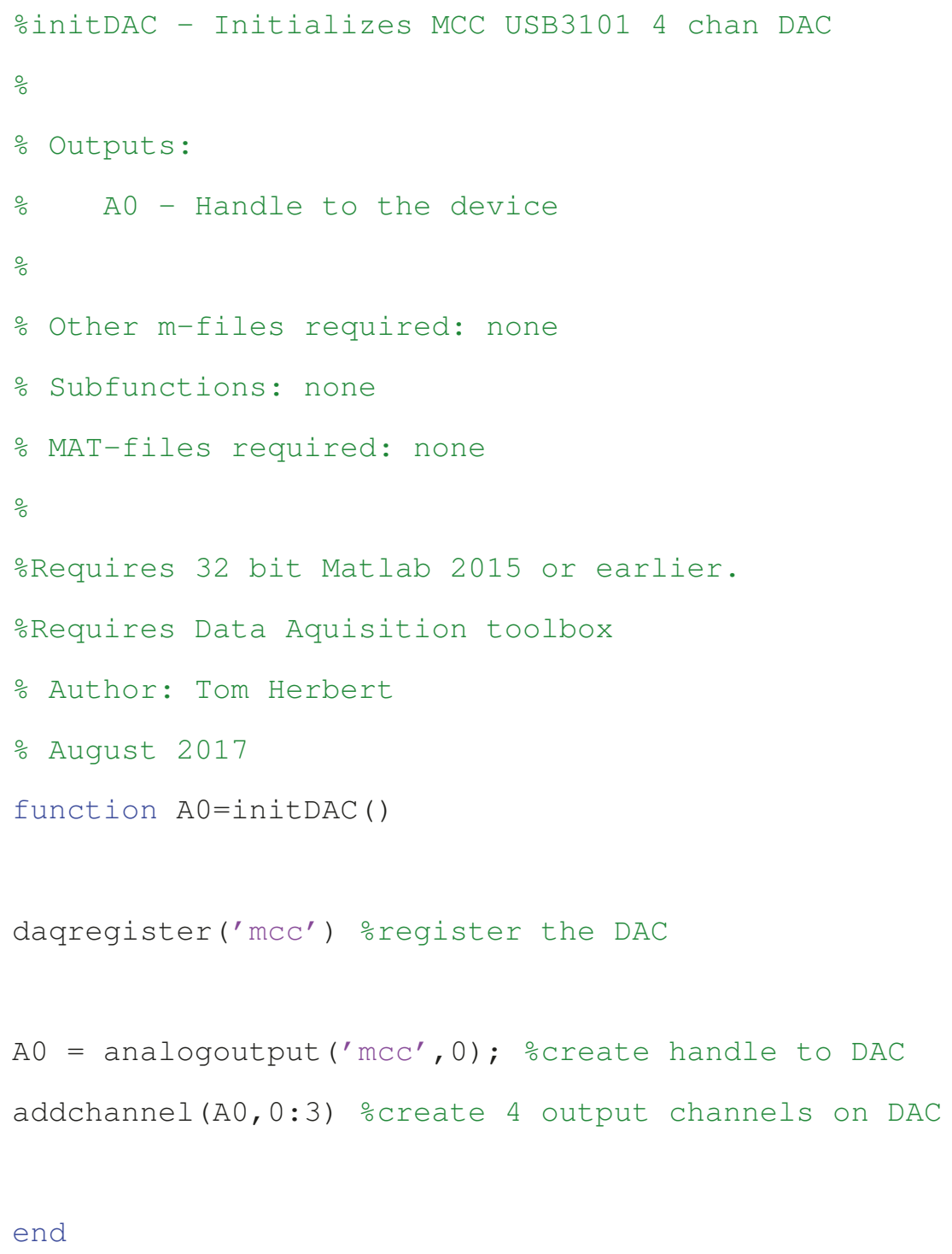




\section{B.7 init_34401A.m}

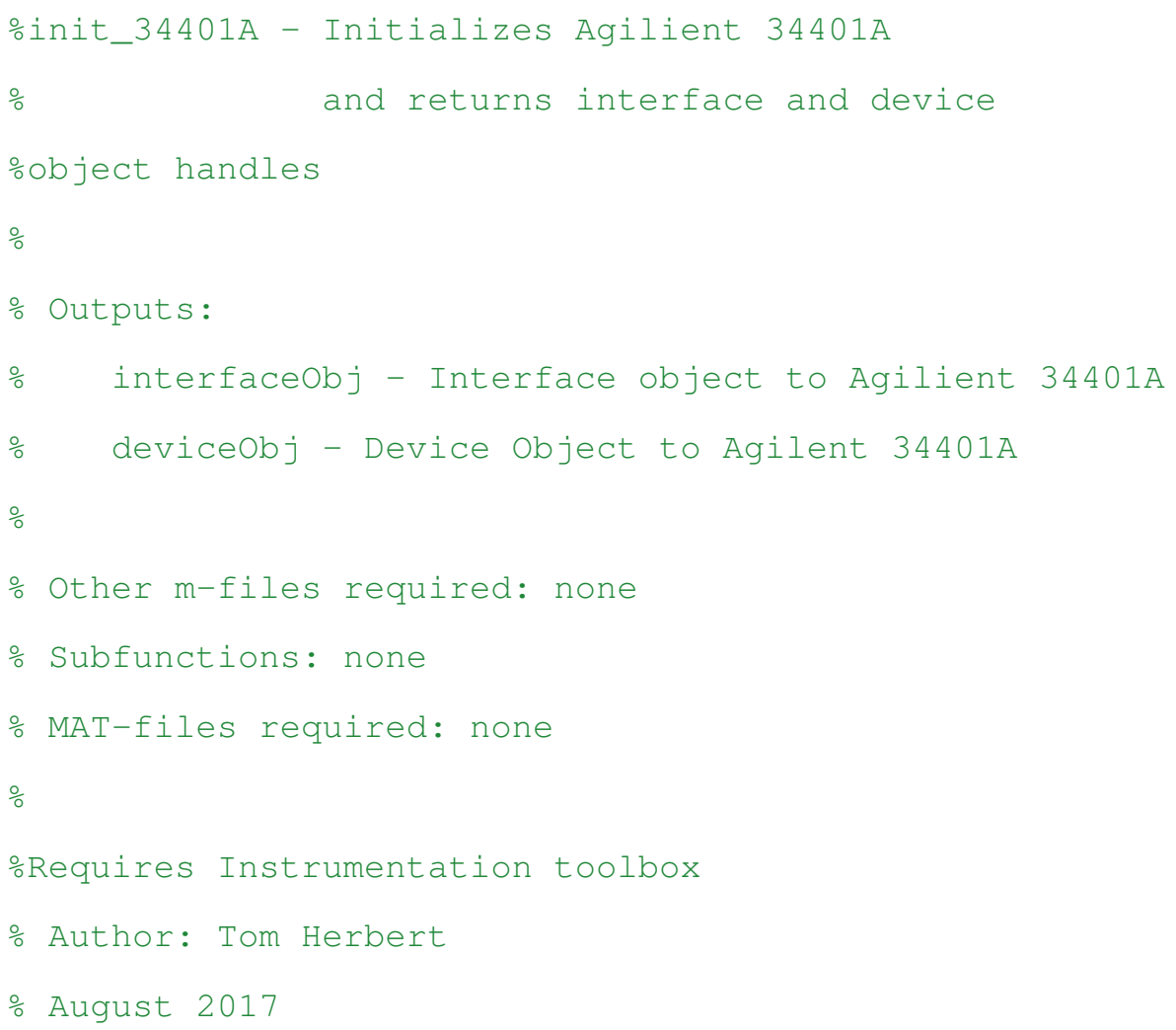




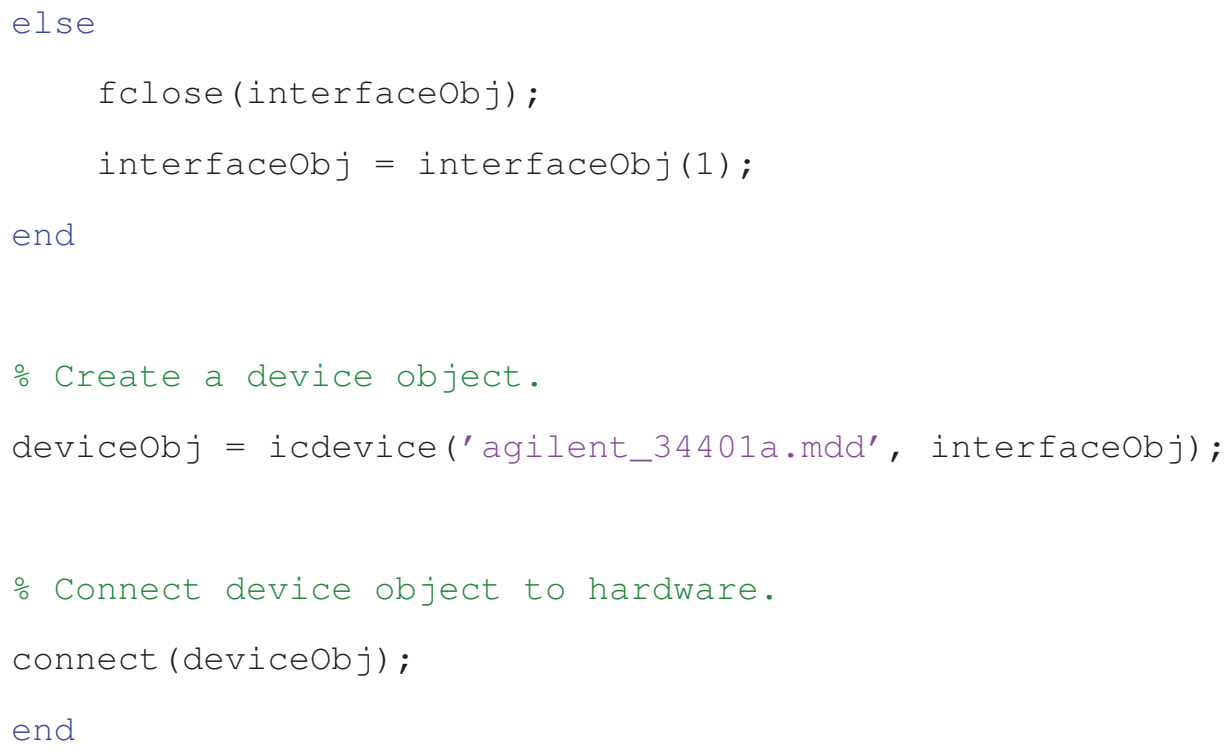

\section{B.8 read_34401A.m}

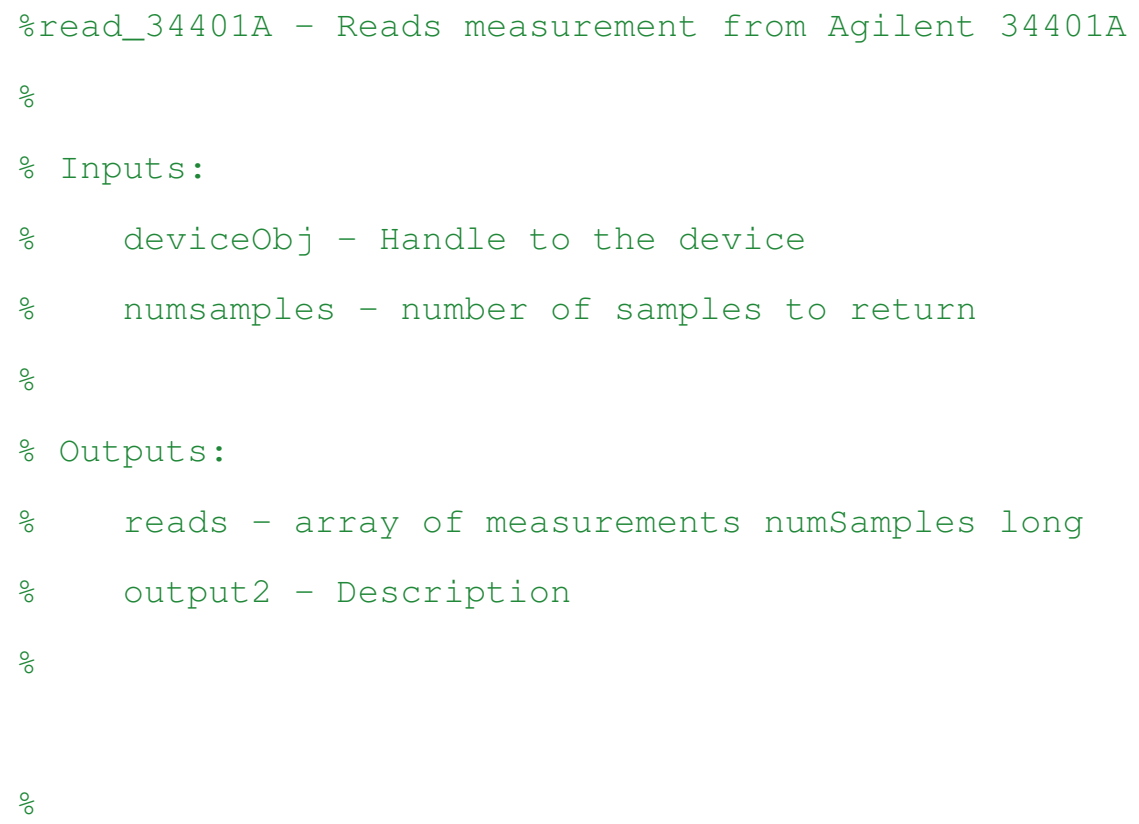




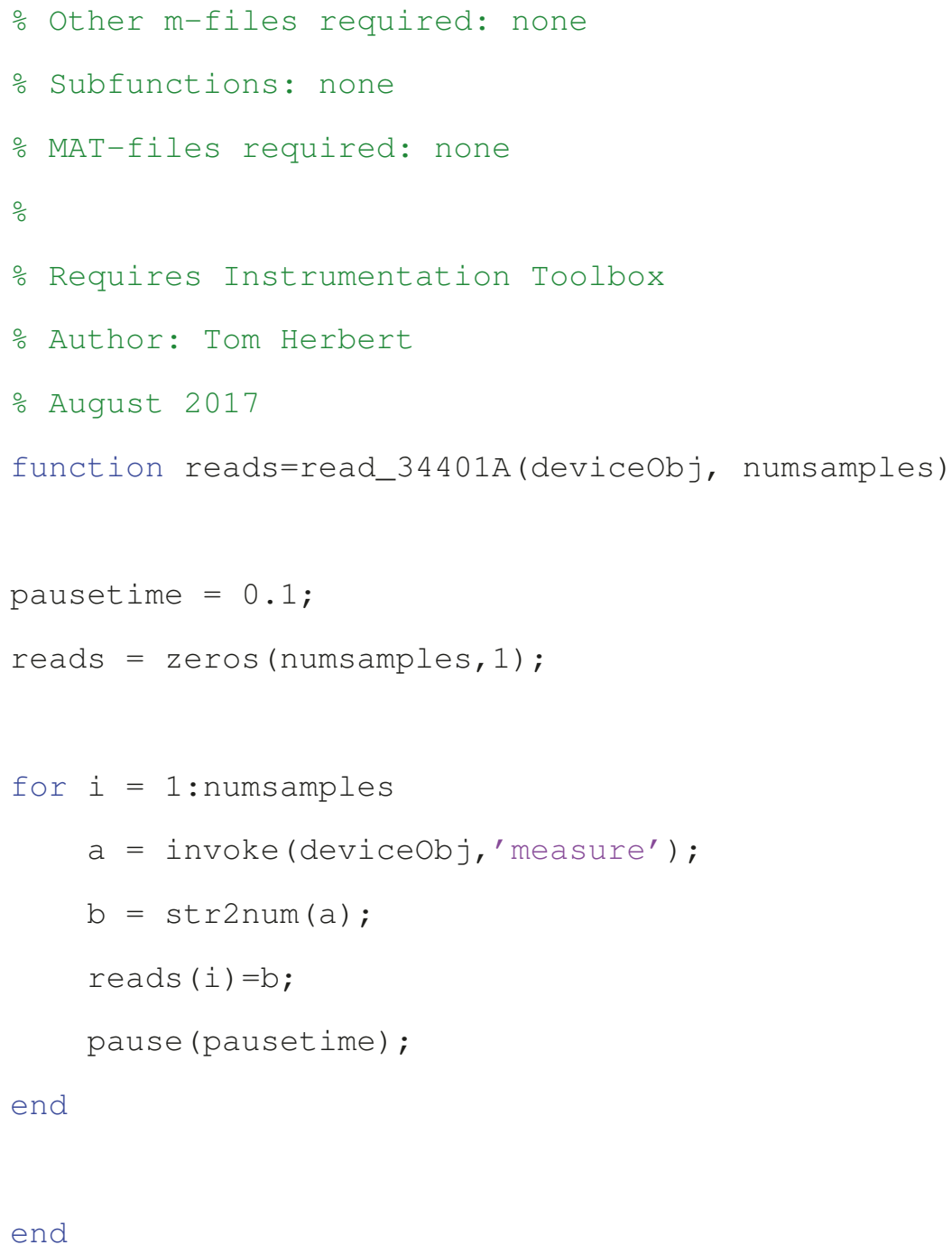

\section{B.9 dataViewer_freq2.m}

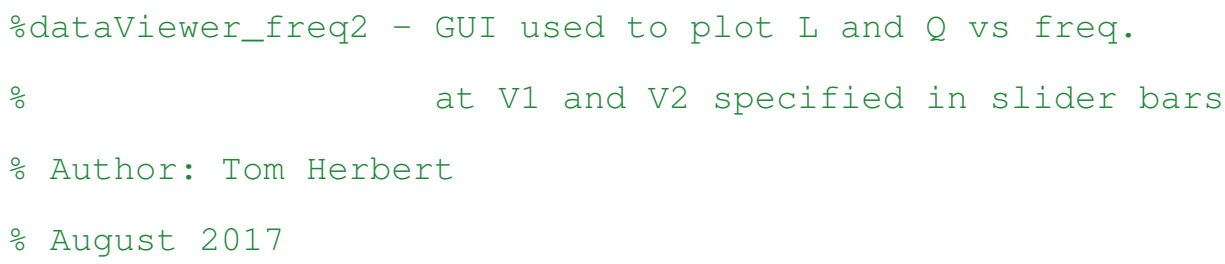




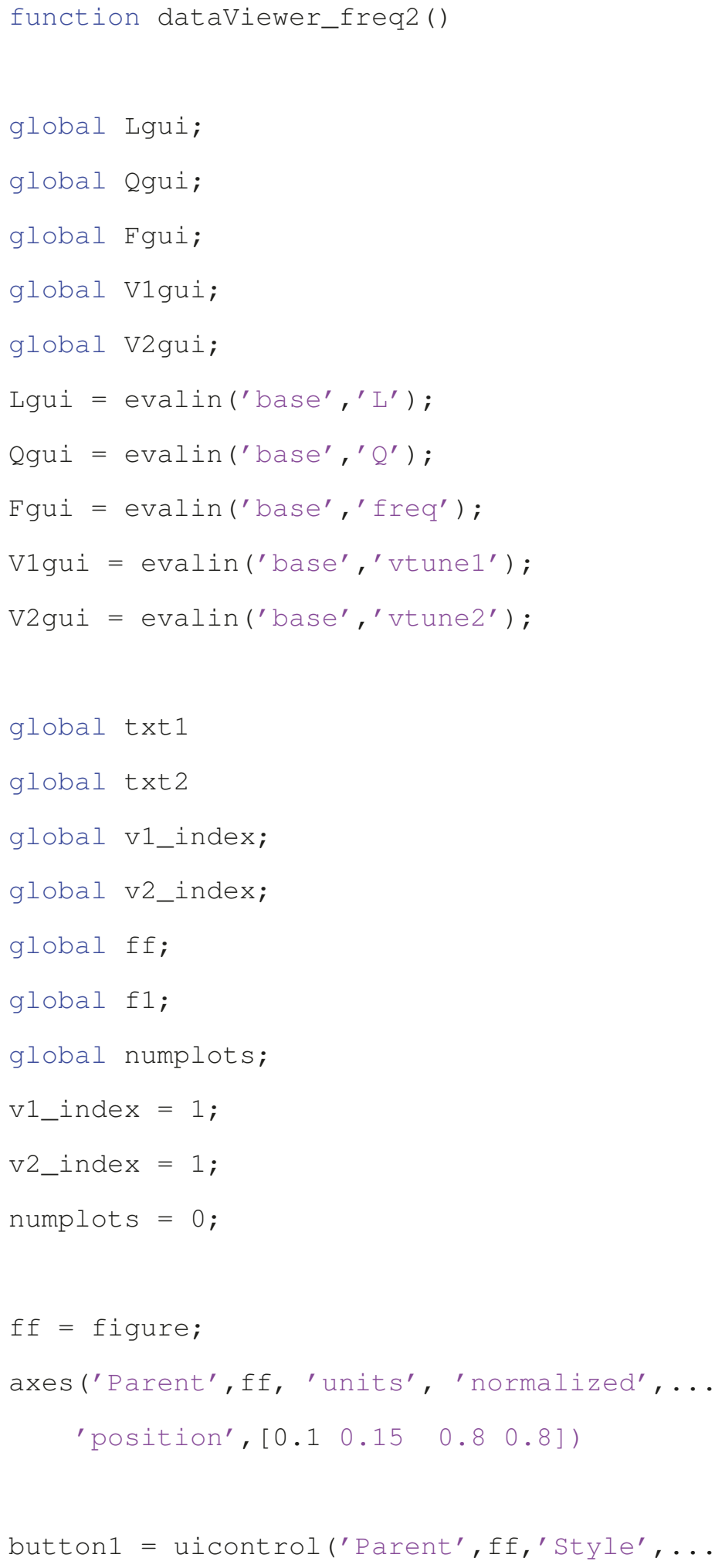









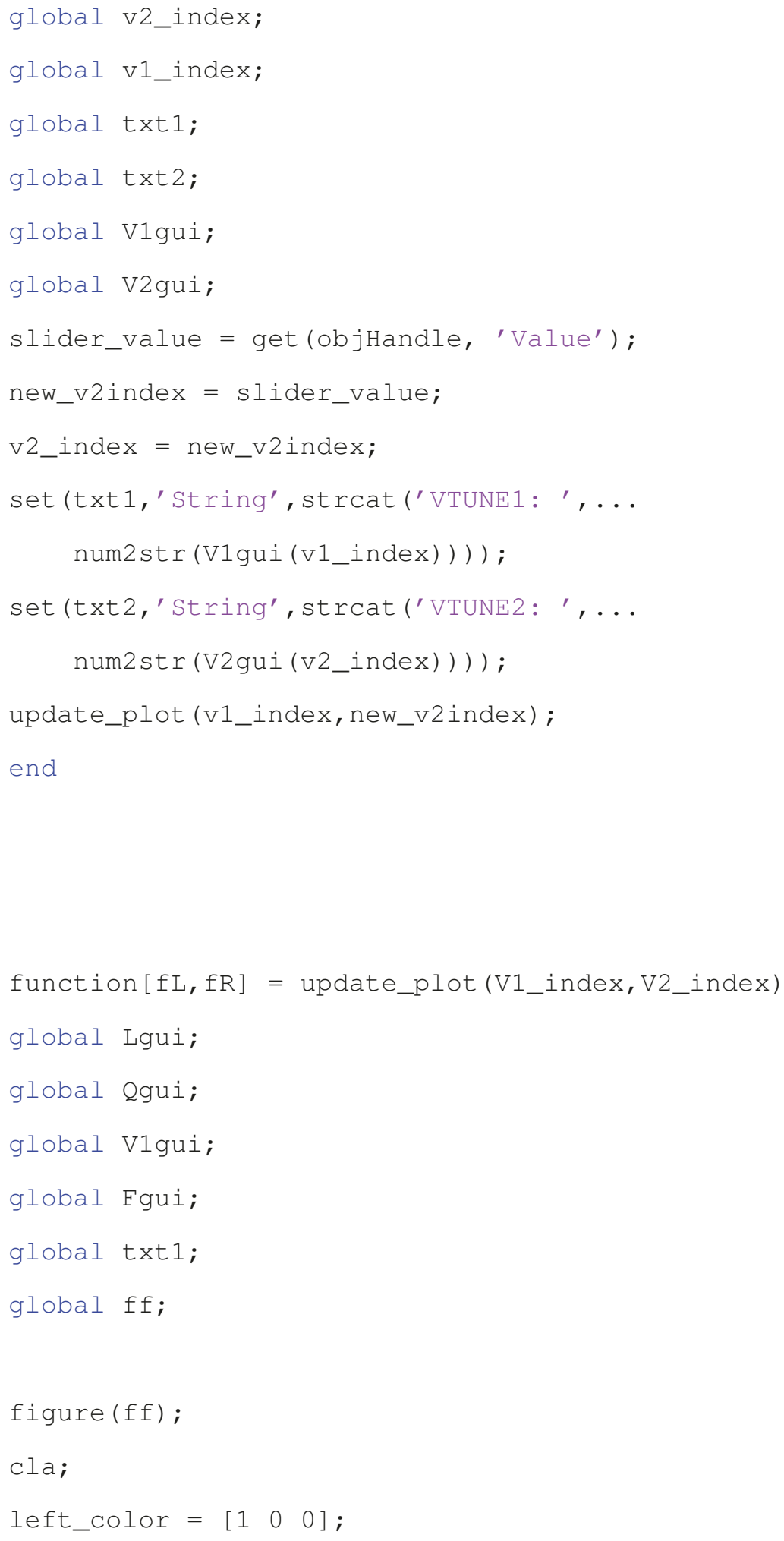




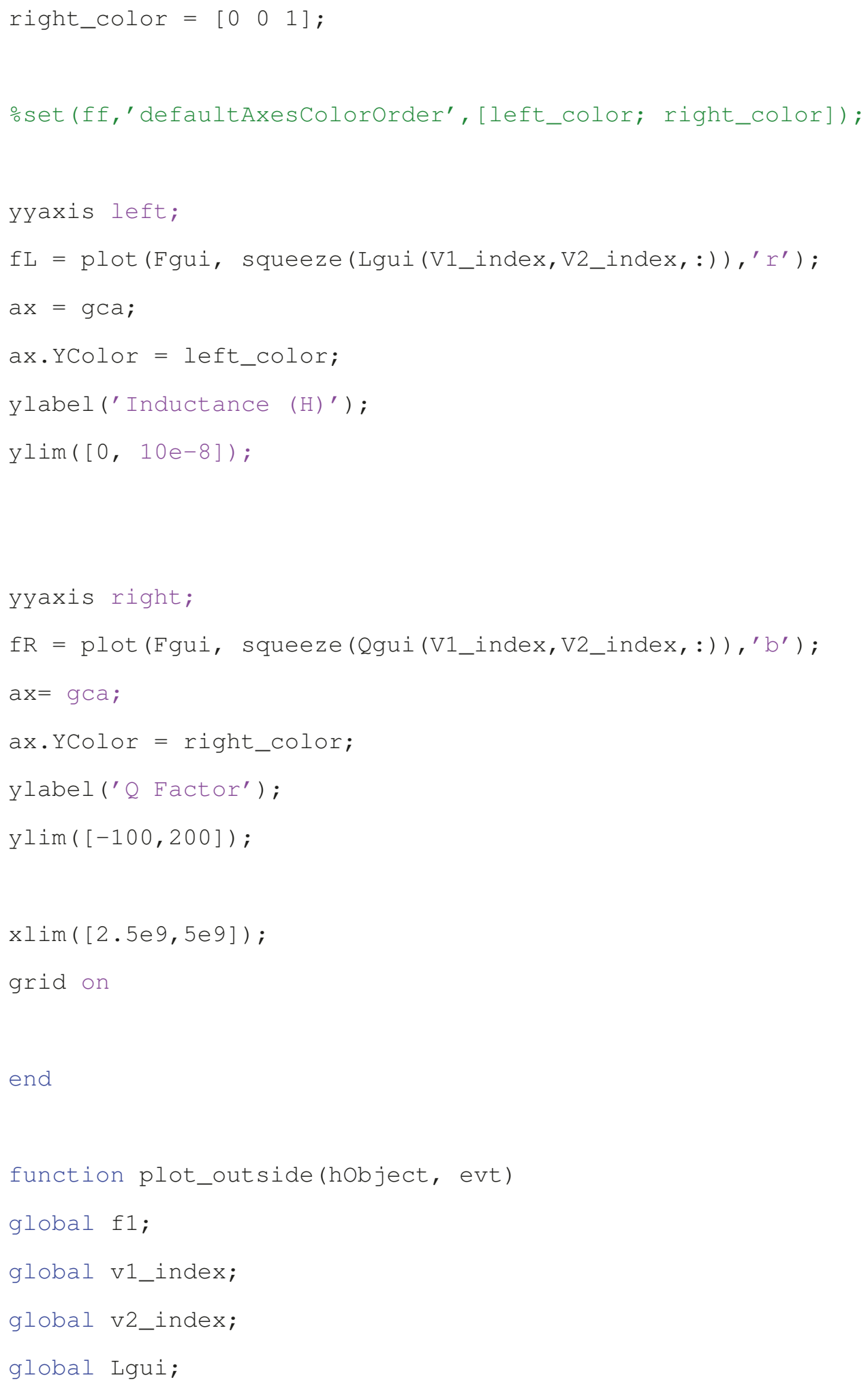




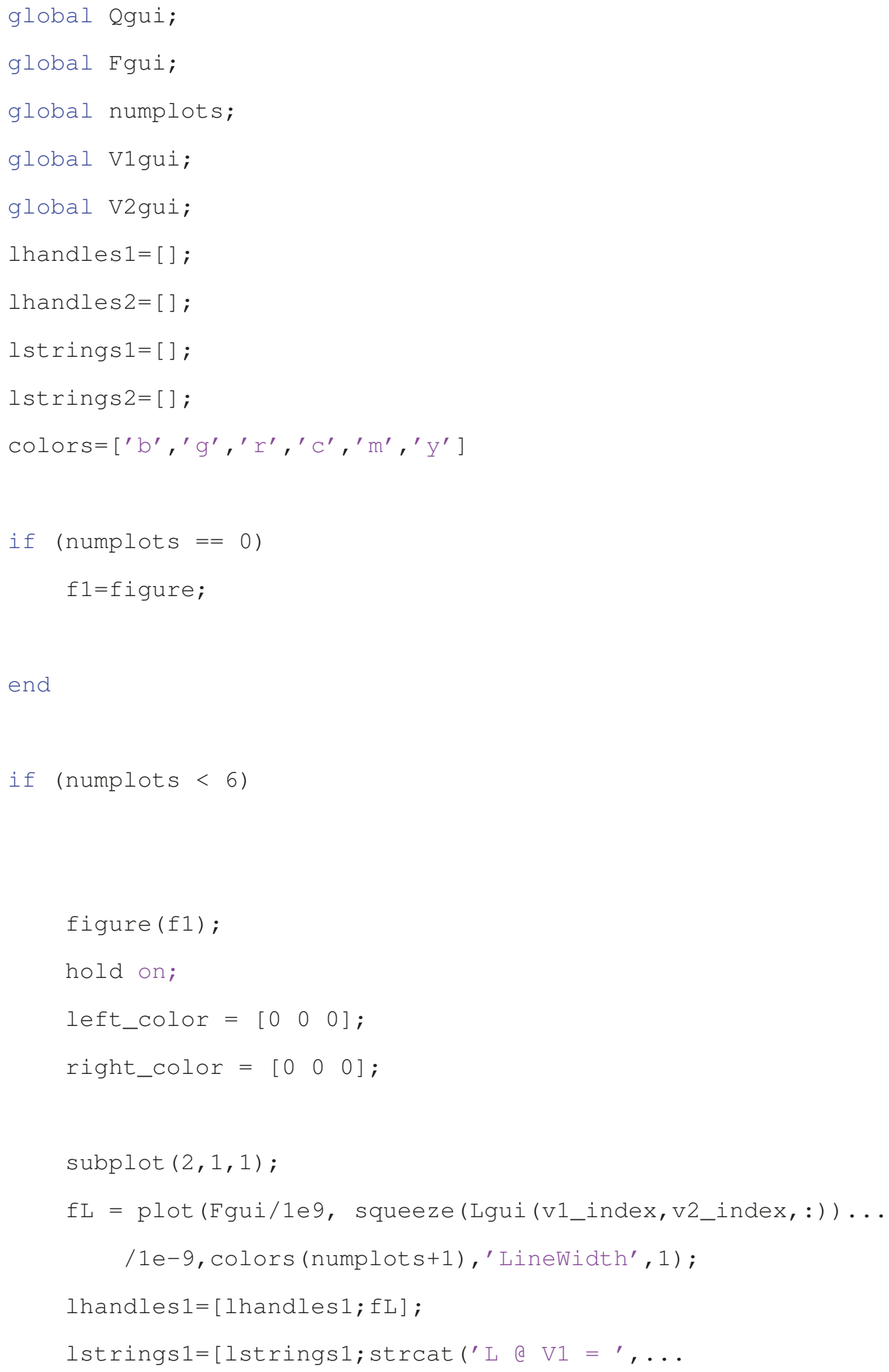




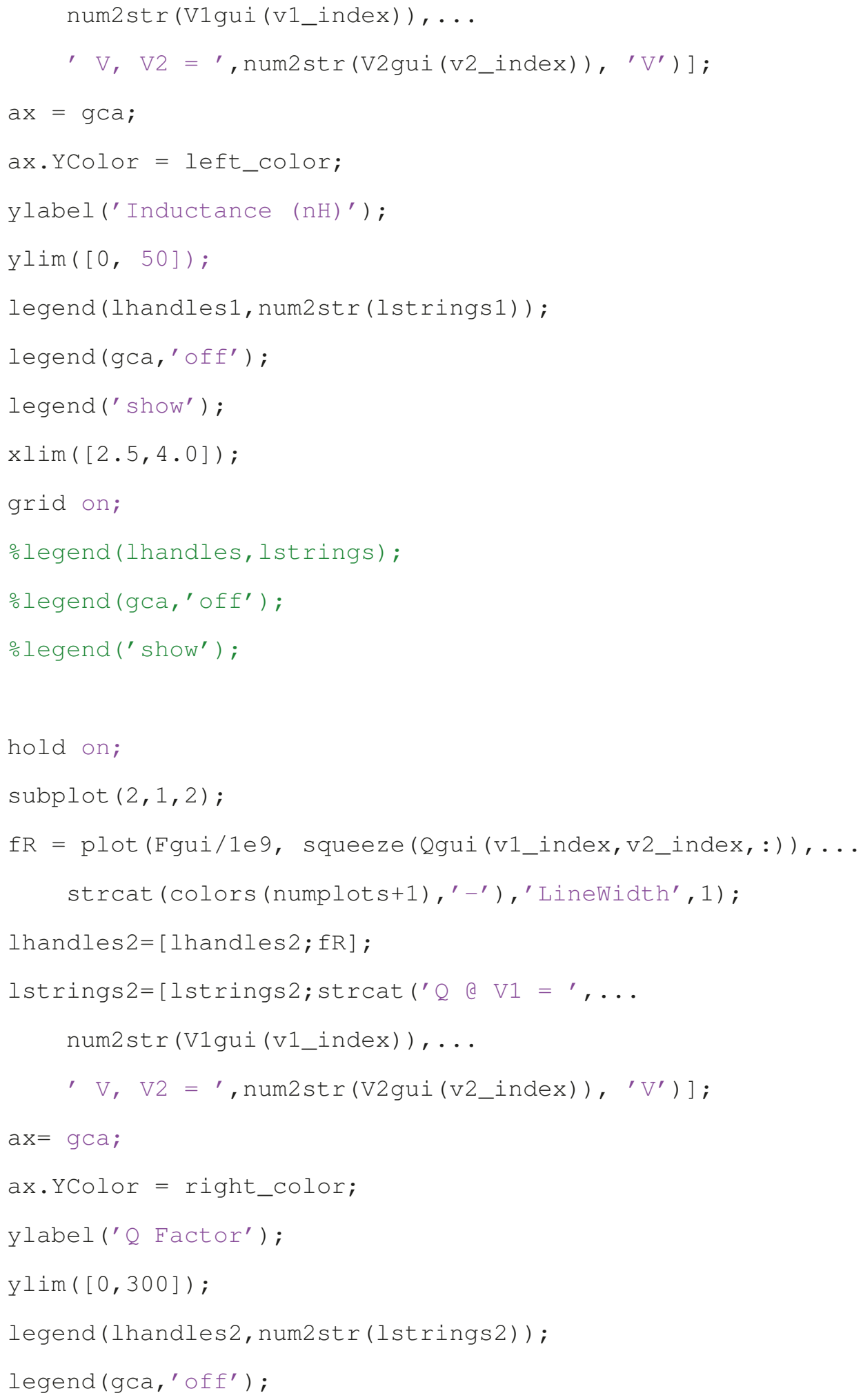




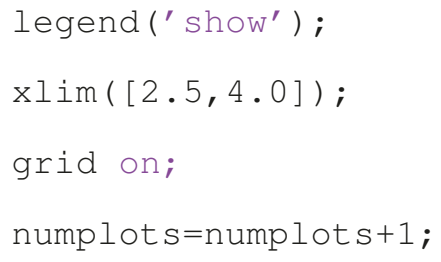

\section{B.10 dataViewer_VTUNE1.m}

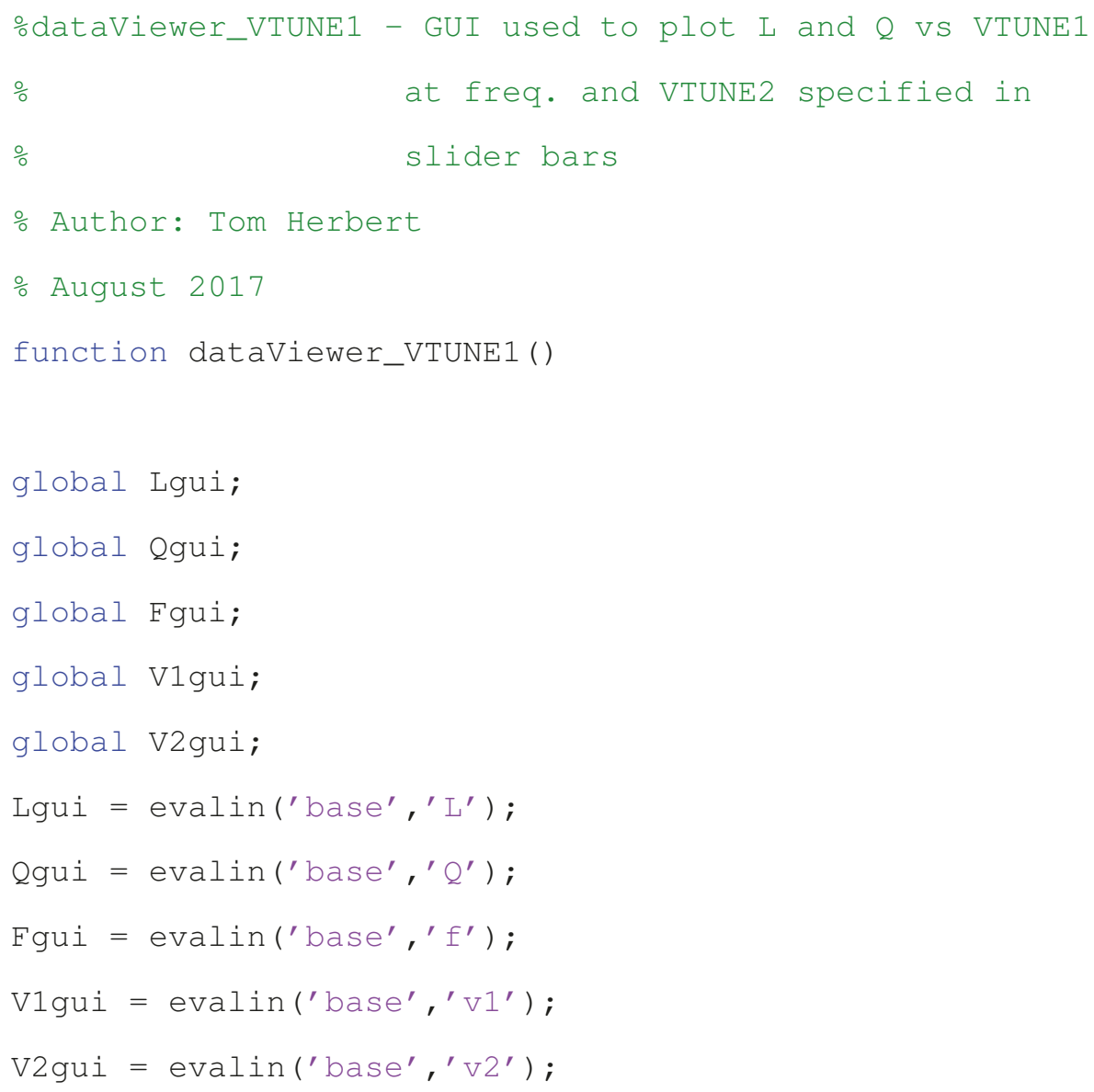




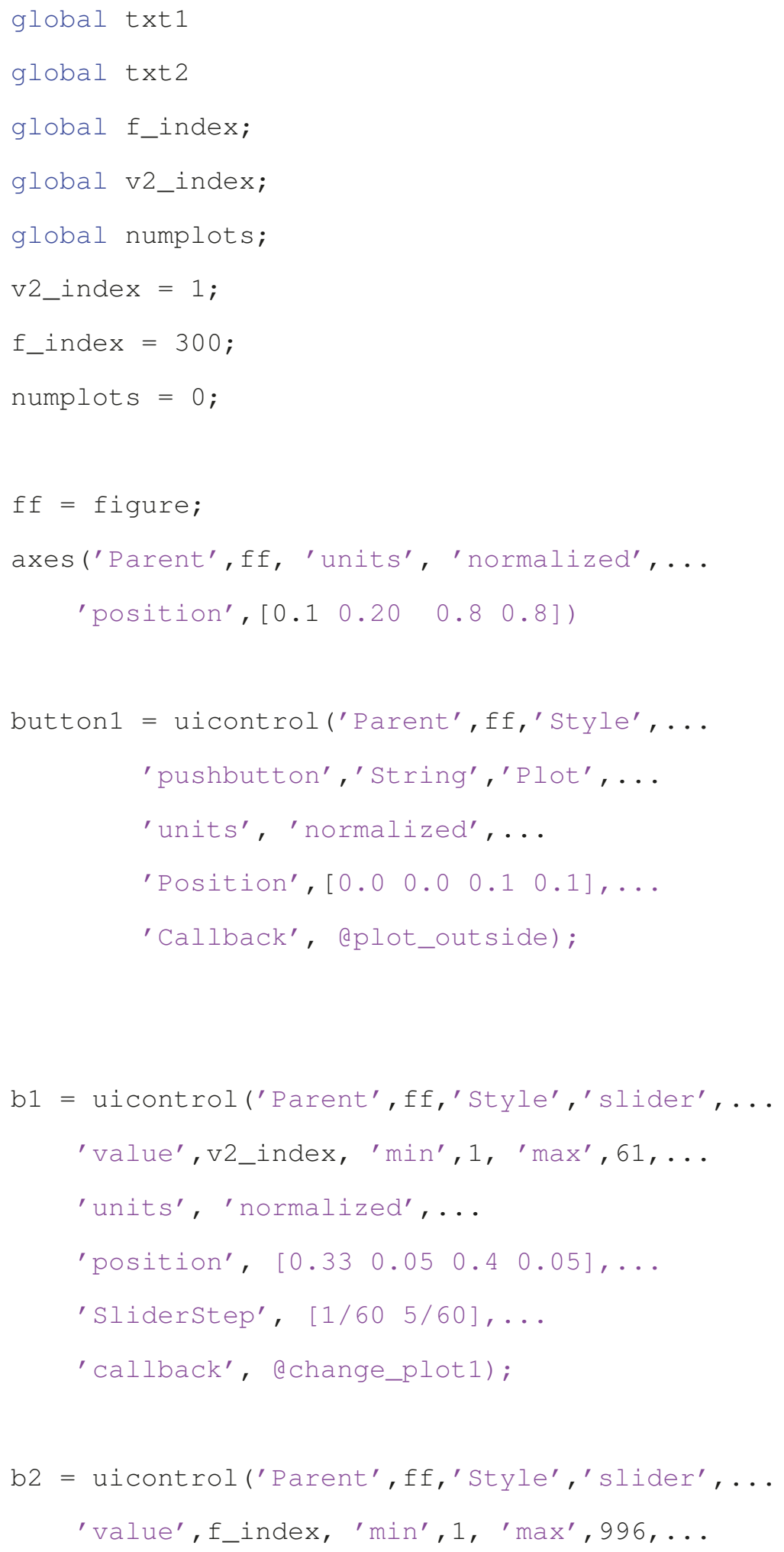




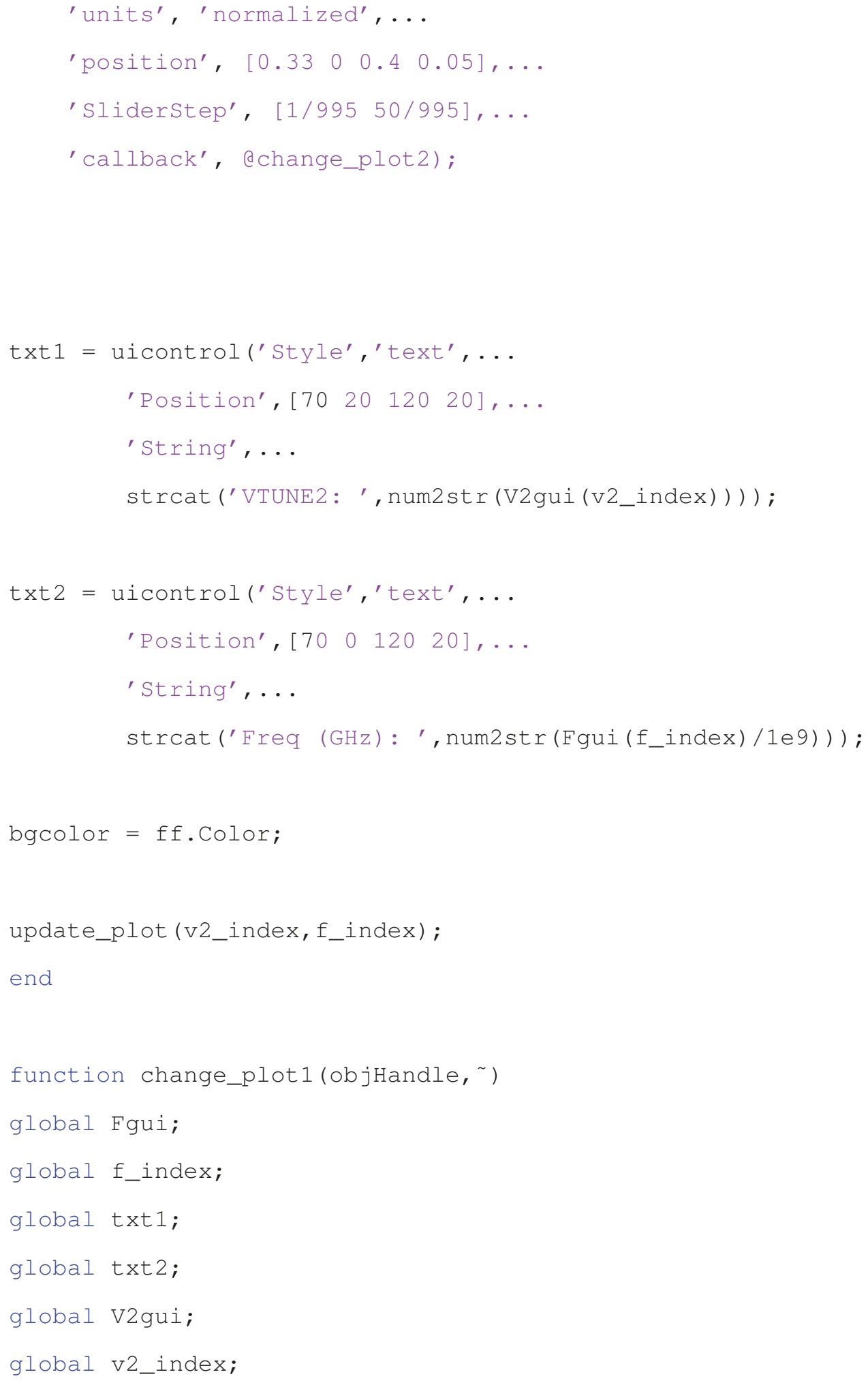




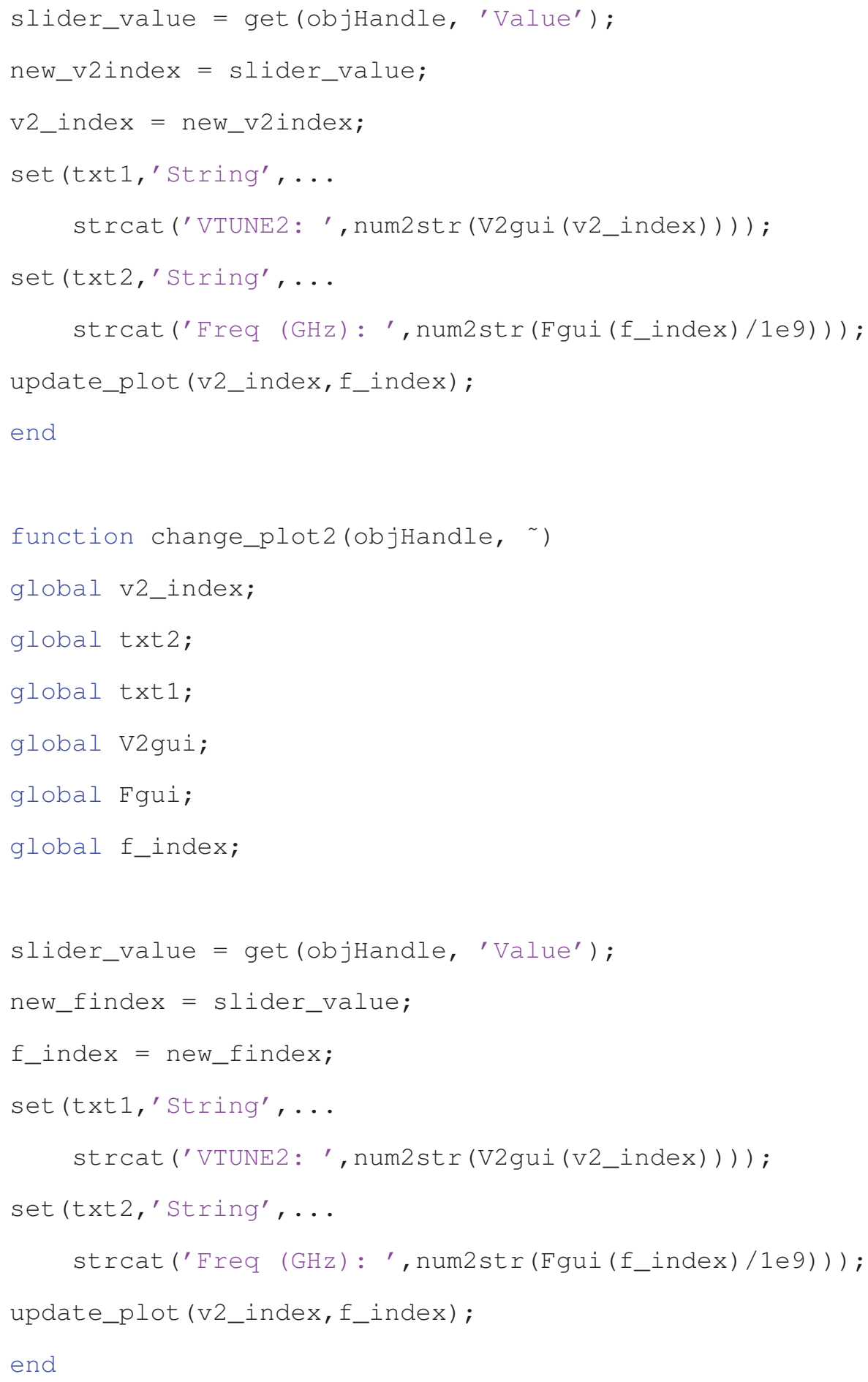




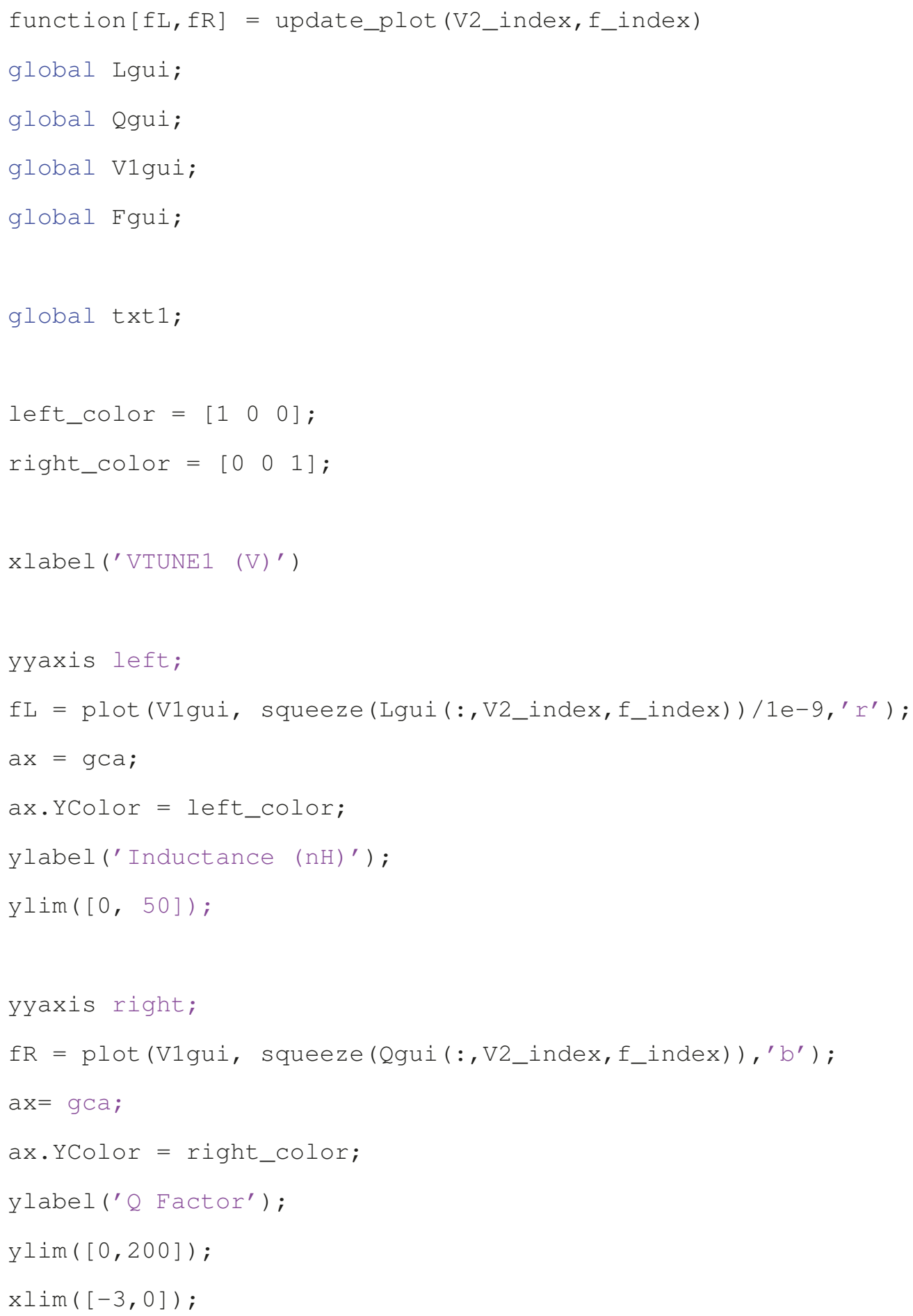




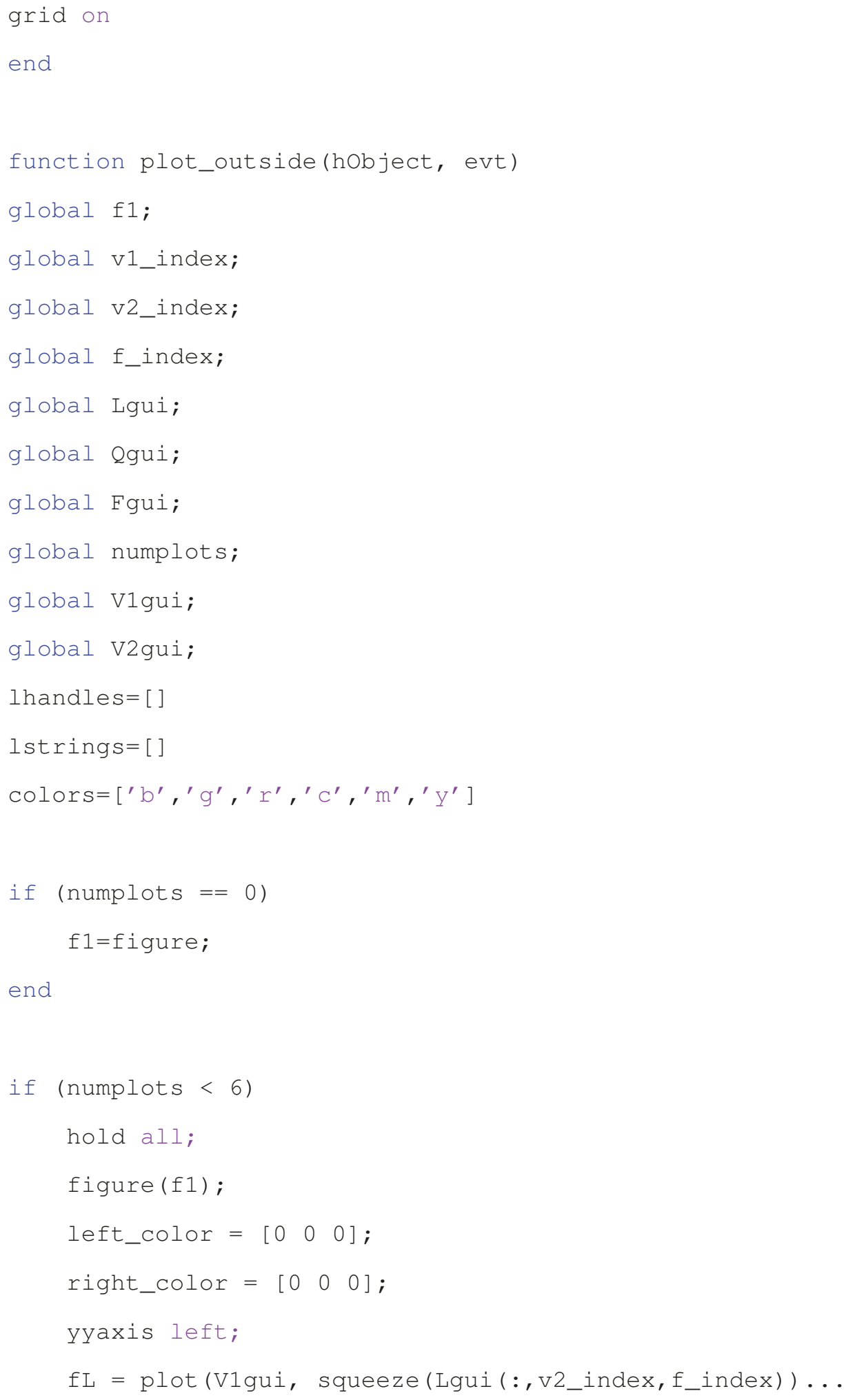




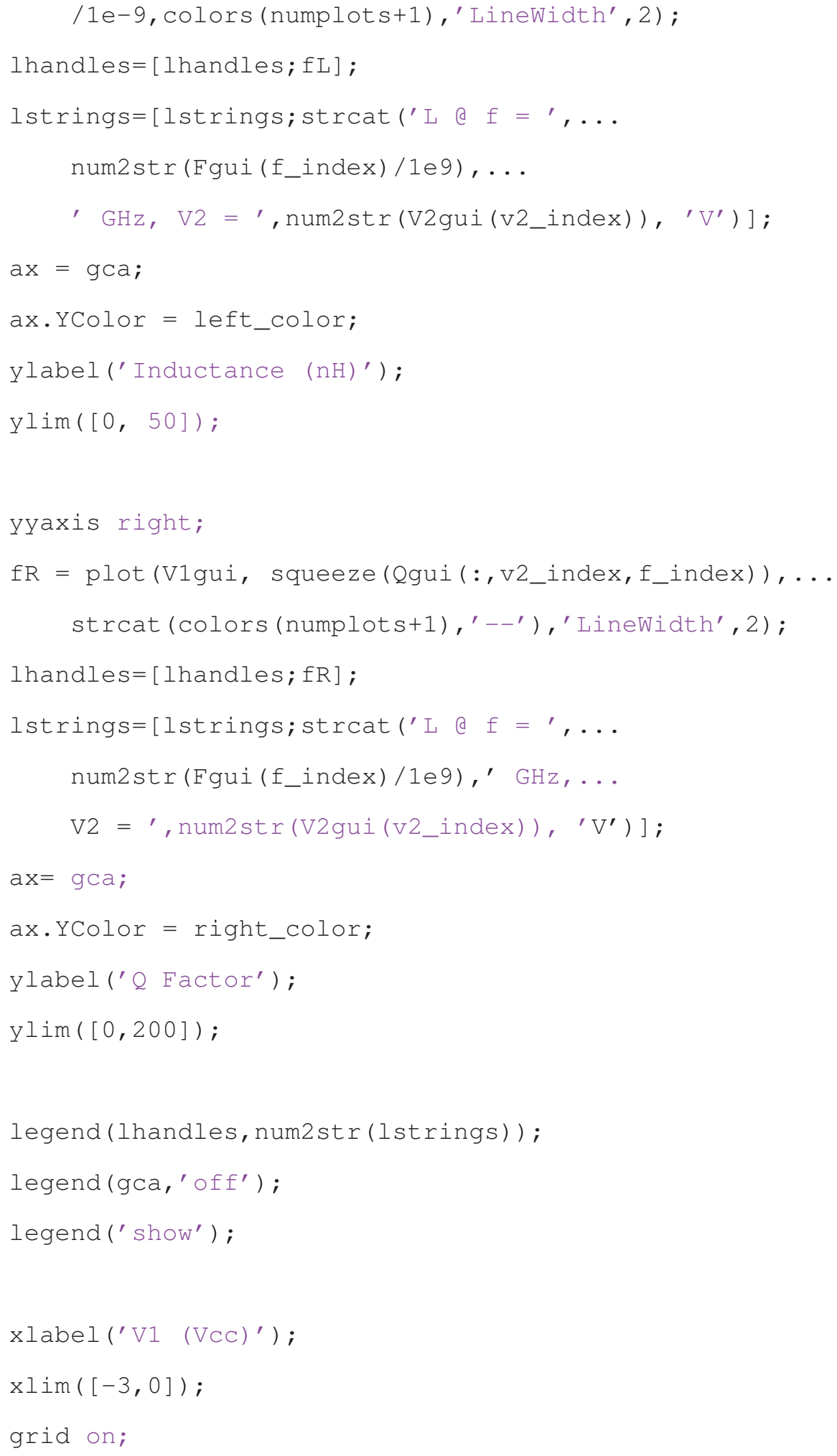


numplot $s=$ numplot $s+1$;

end

end

\section{B.11 dataViewer_VTUNE2.m}

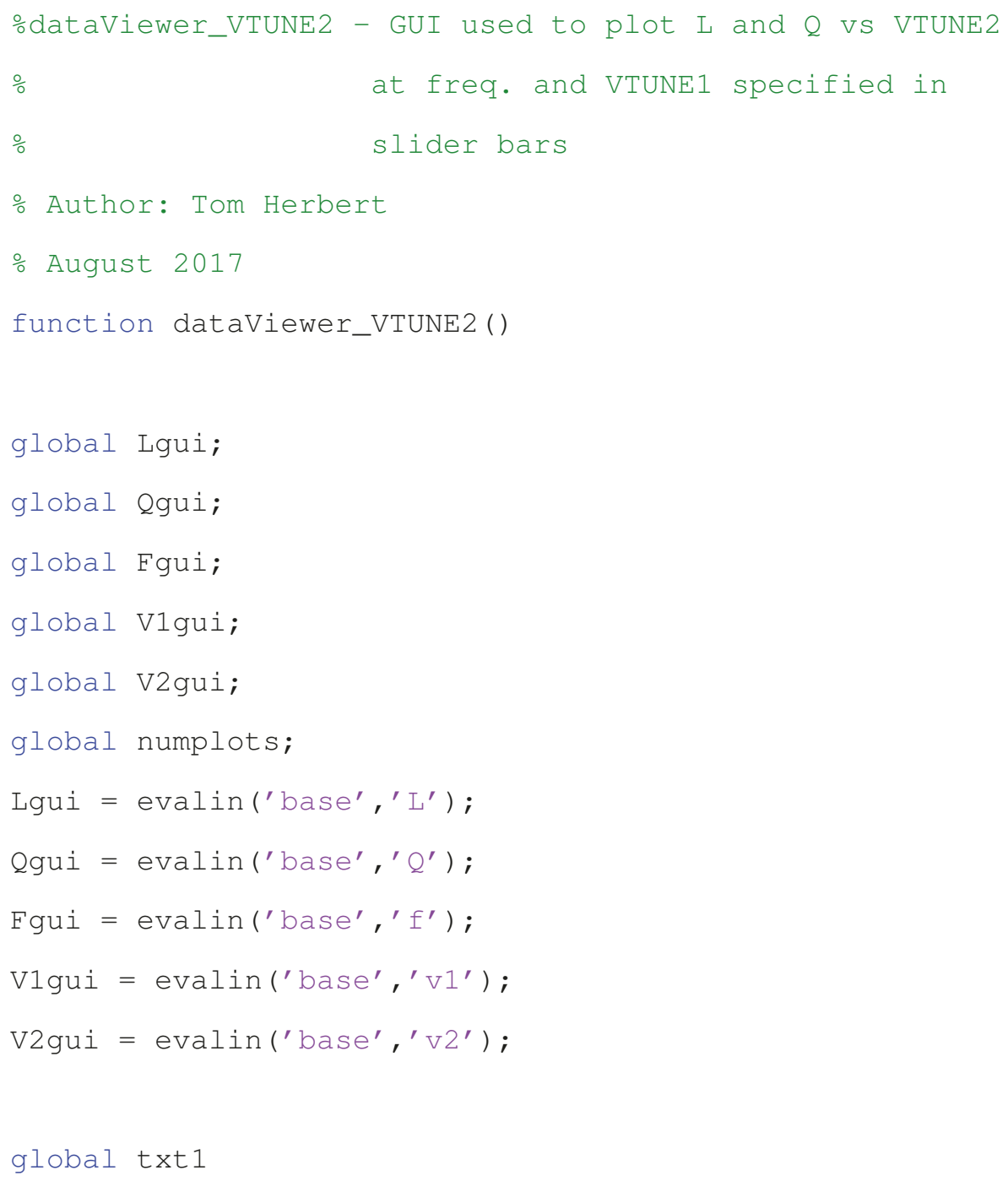




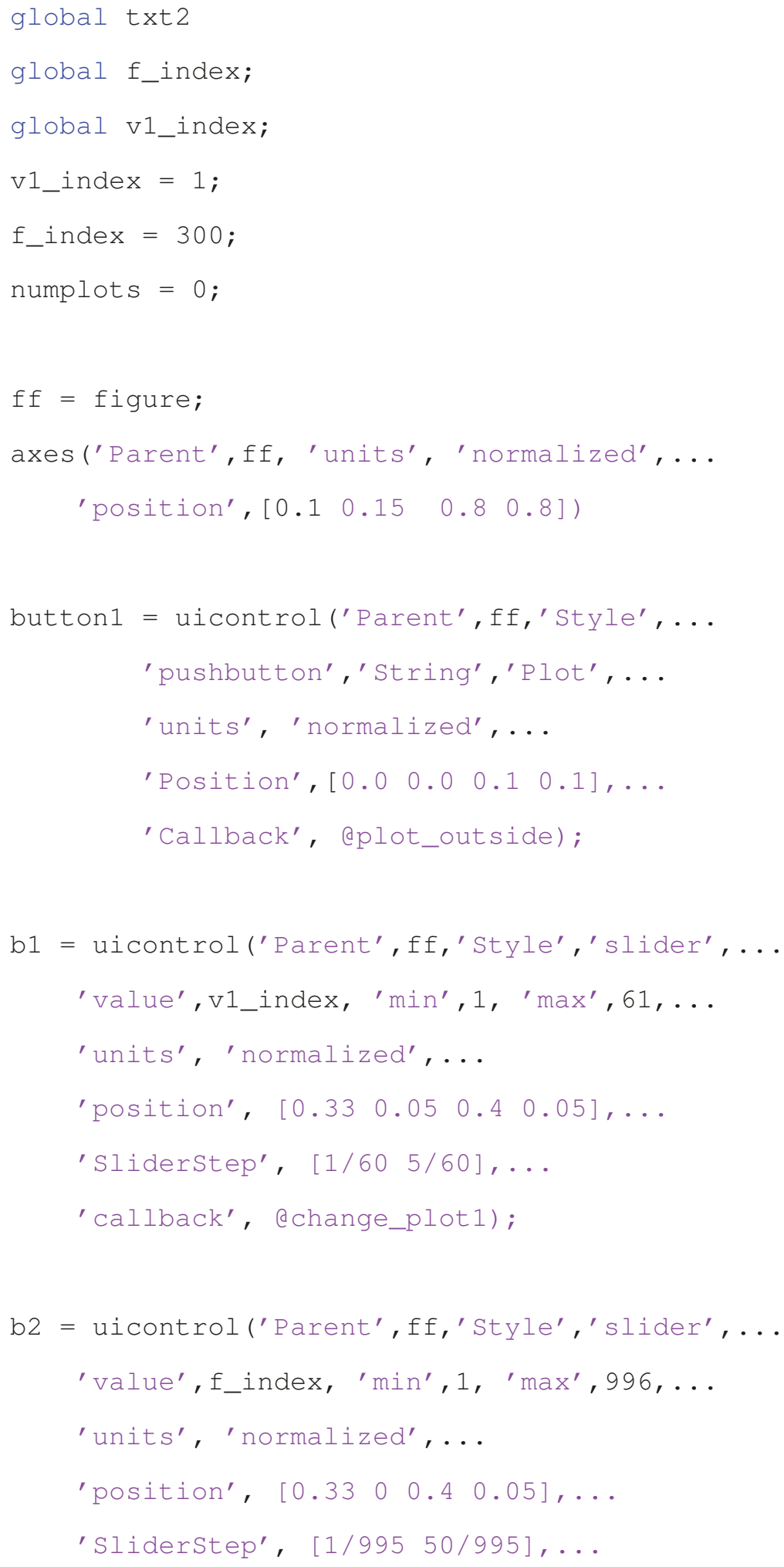


'callback', (cchange_plot2);

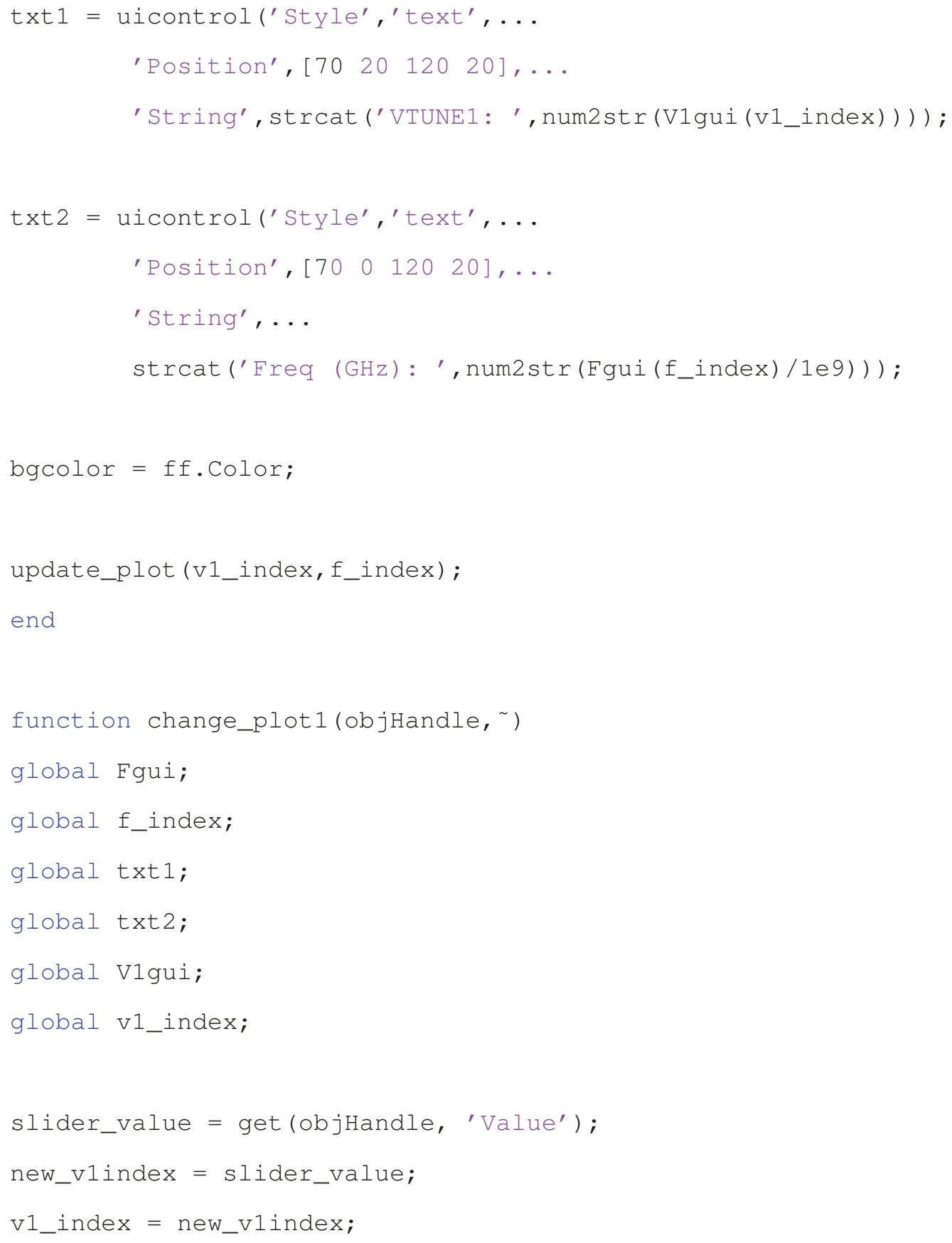




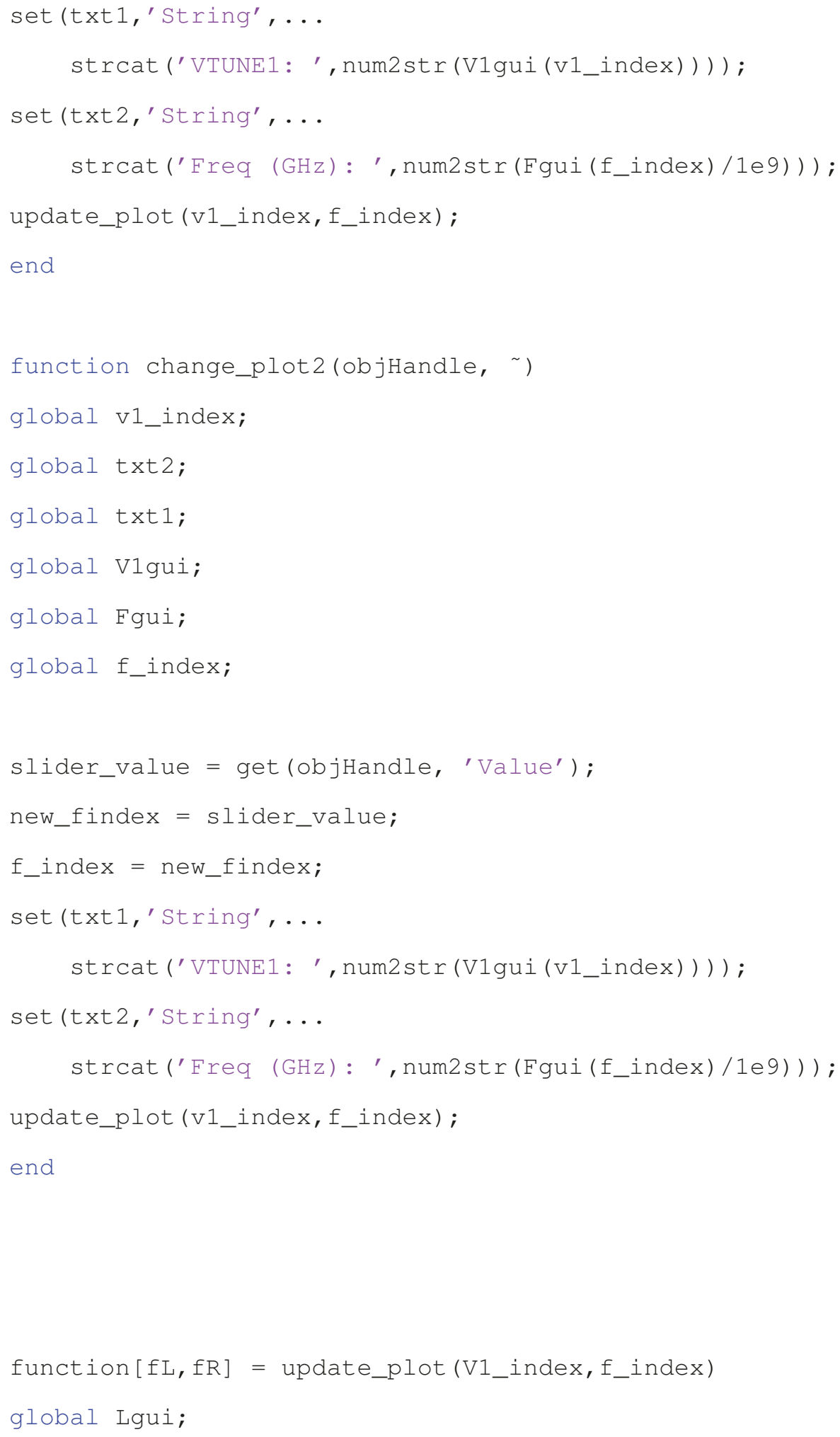




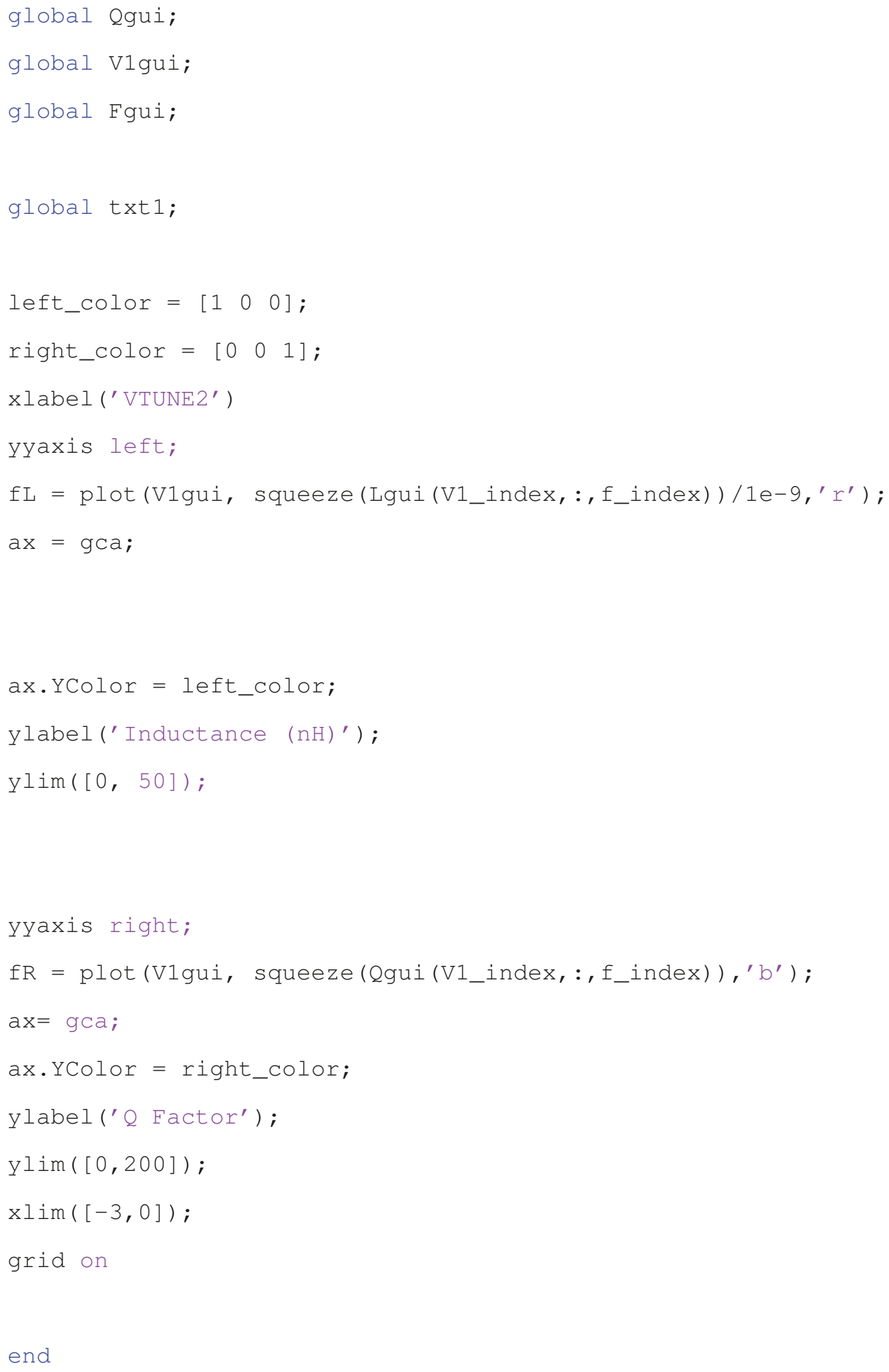




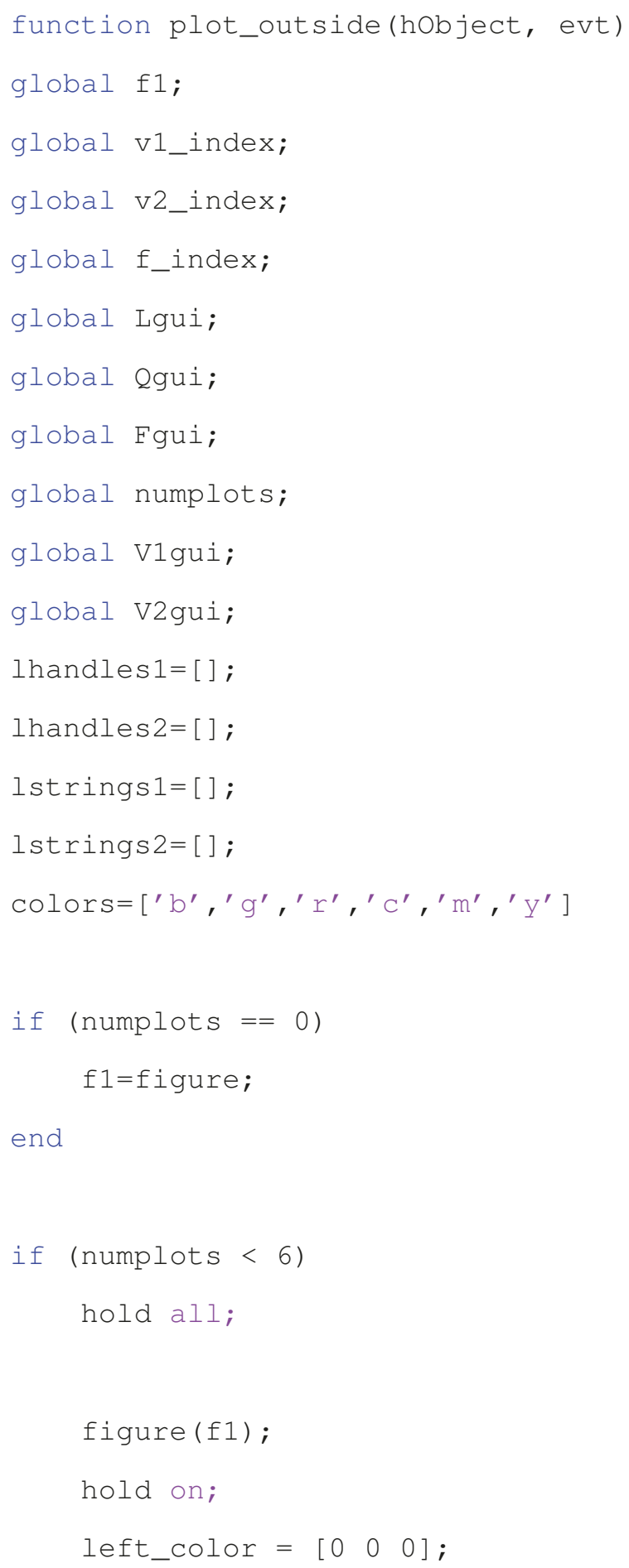




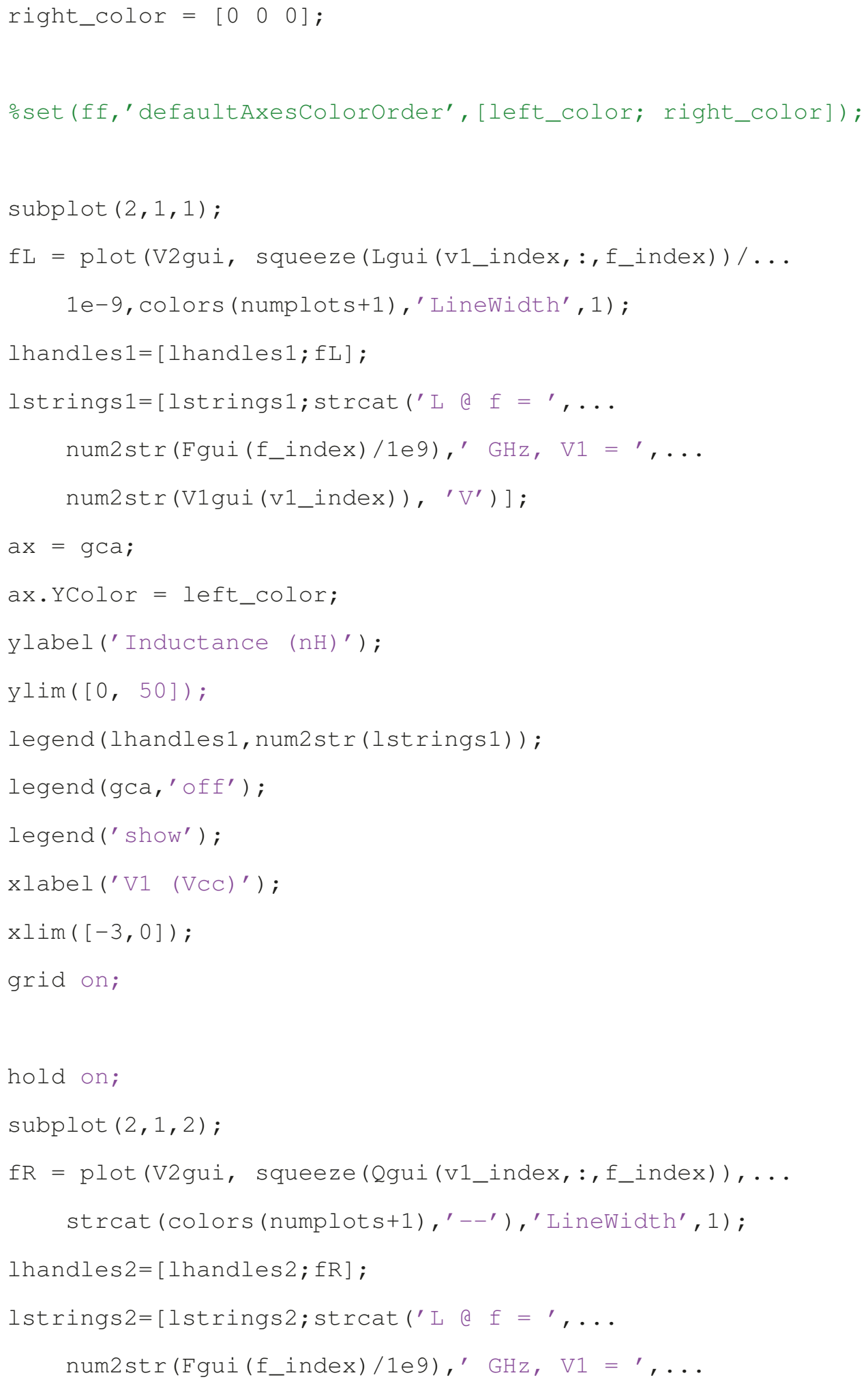




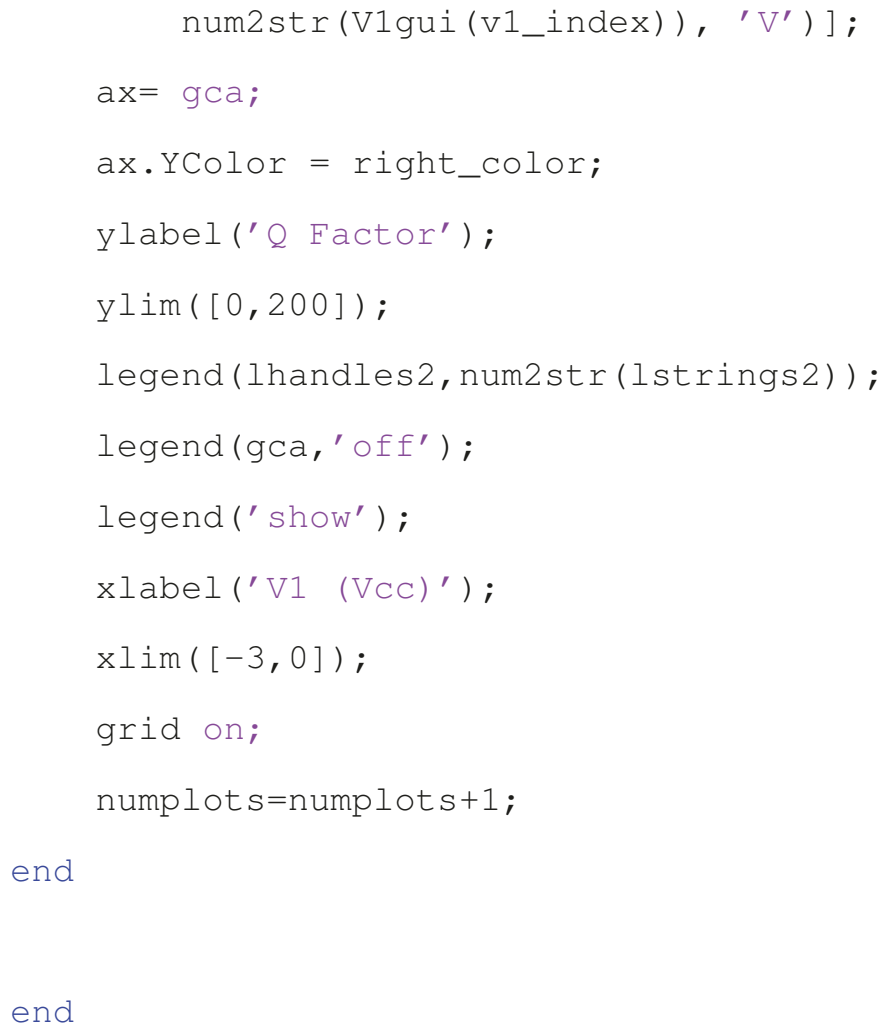

\section{B.12 BPF_Viewer_freq2.m}

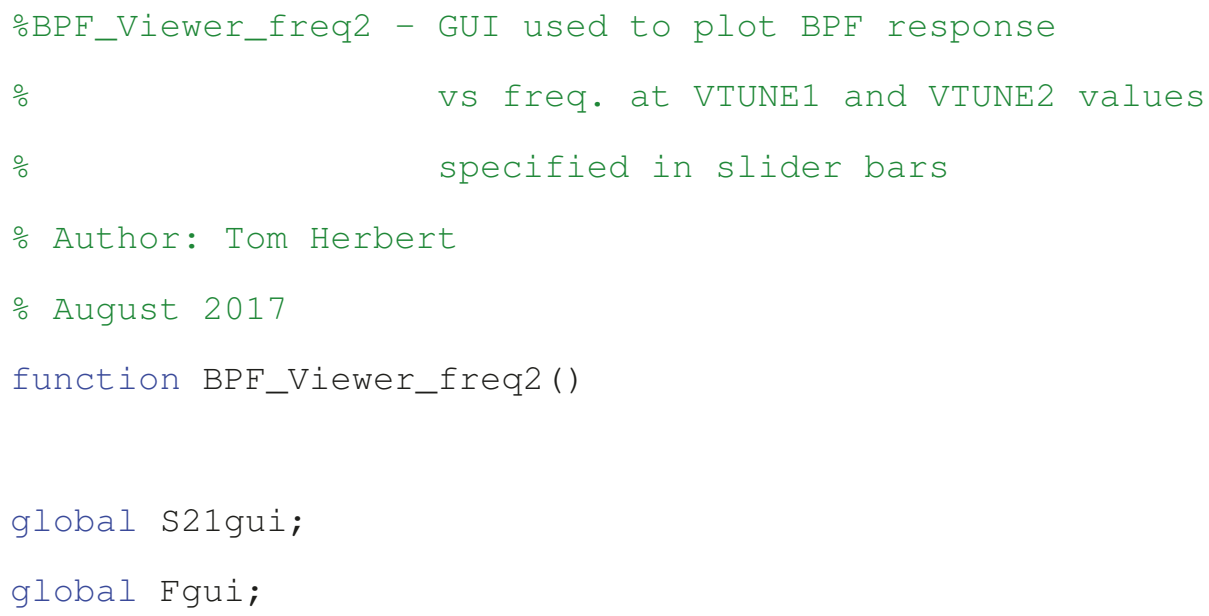




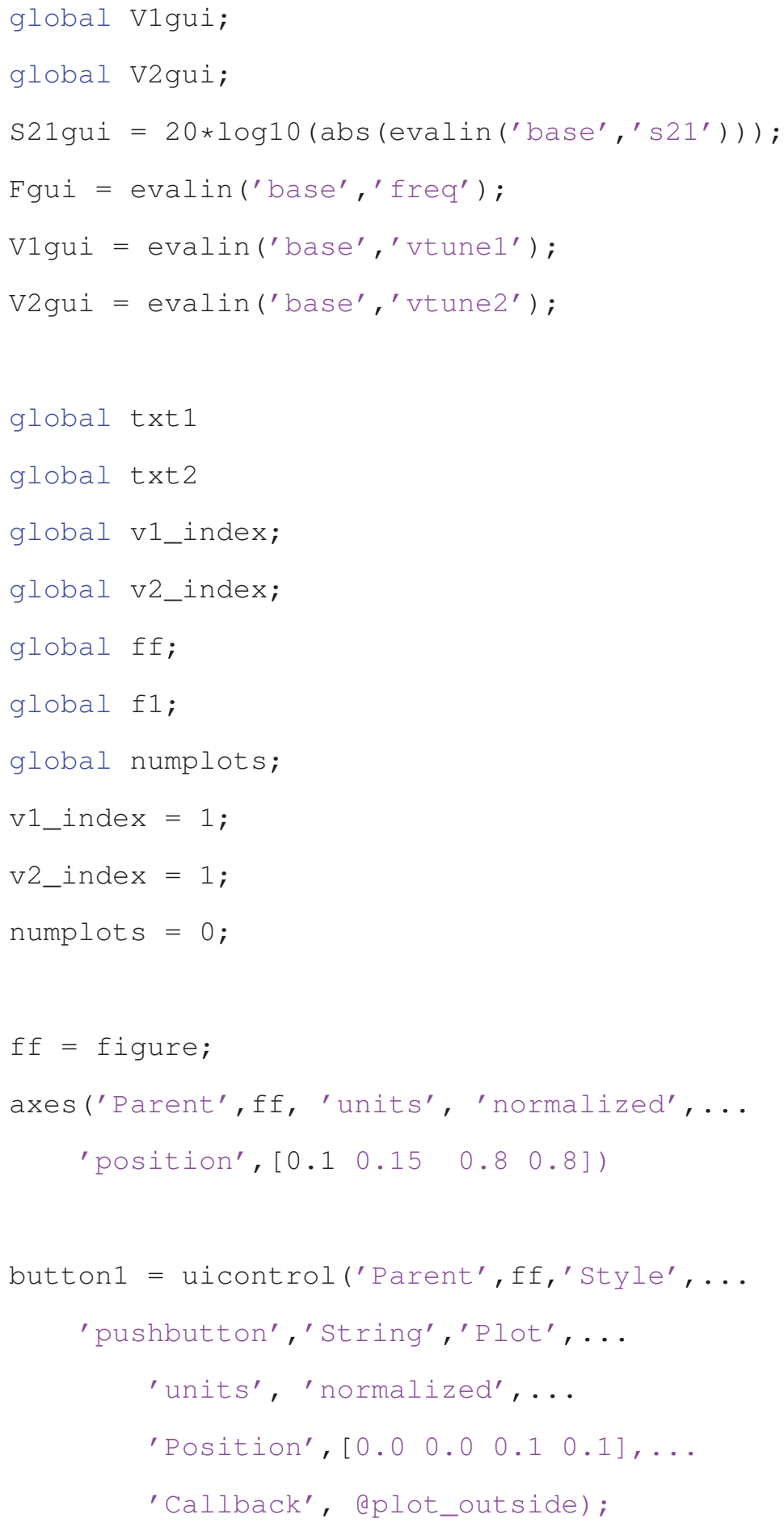




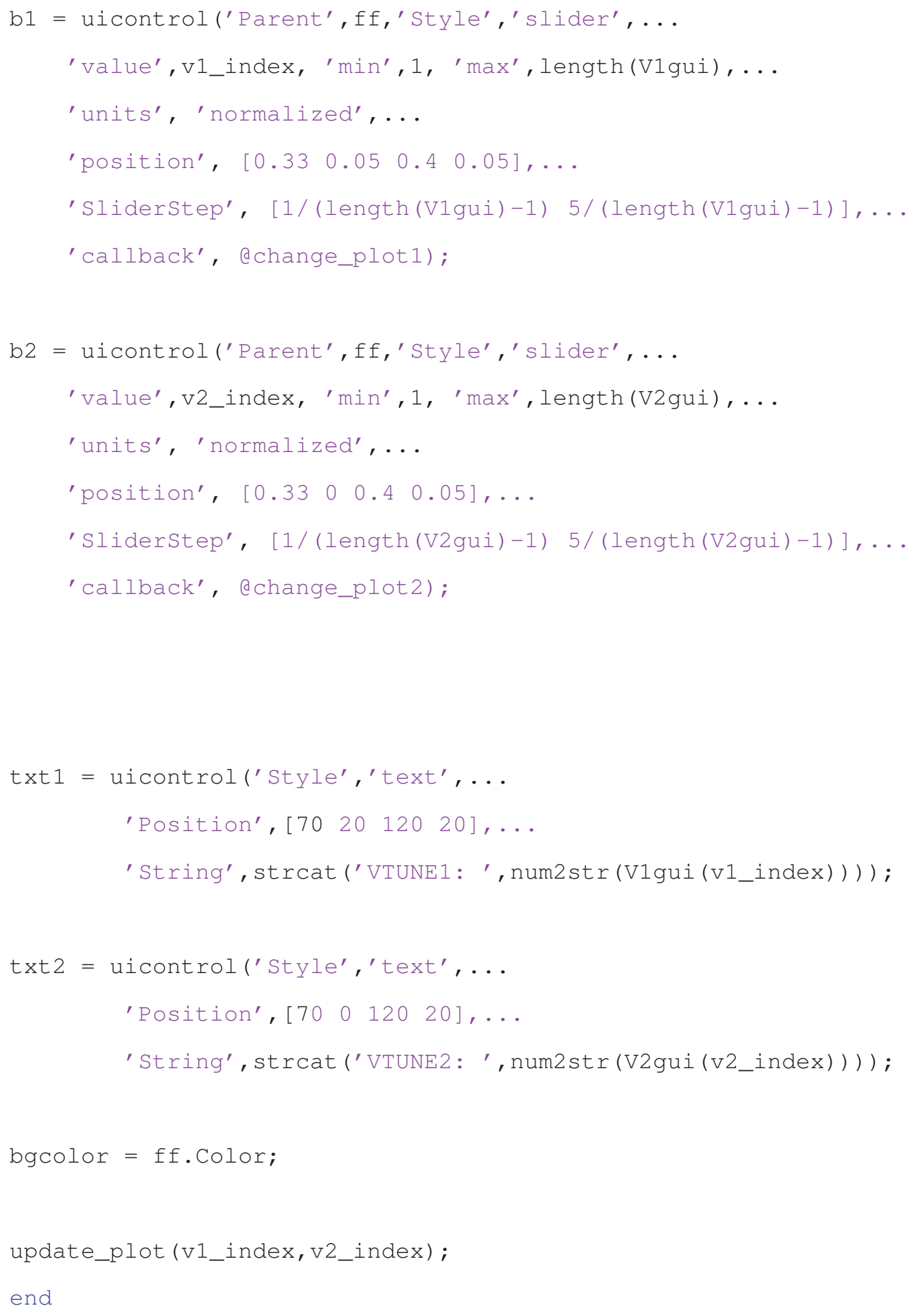




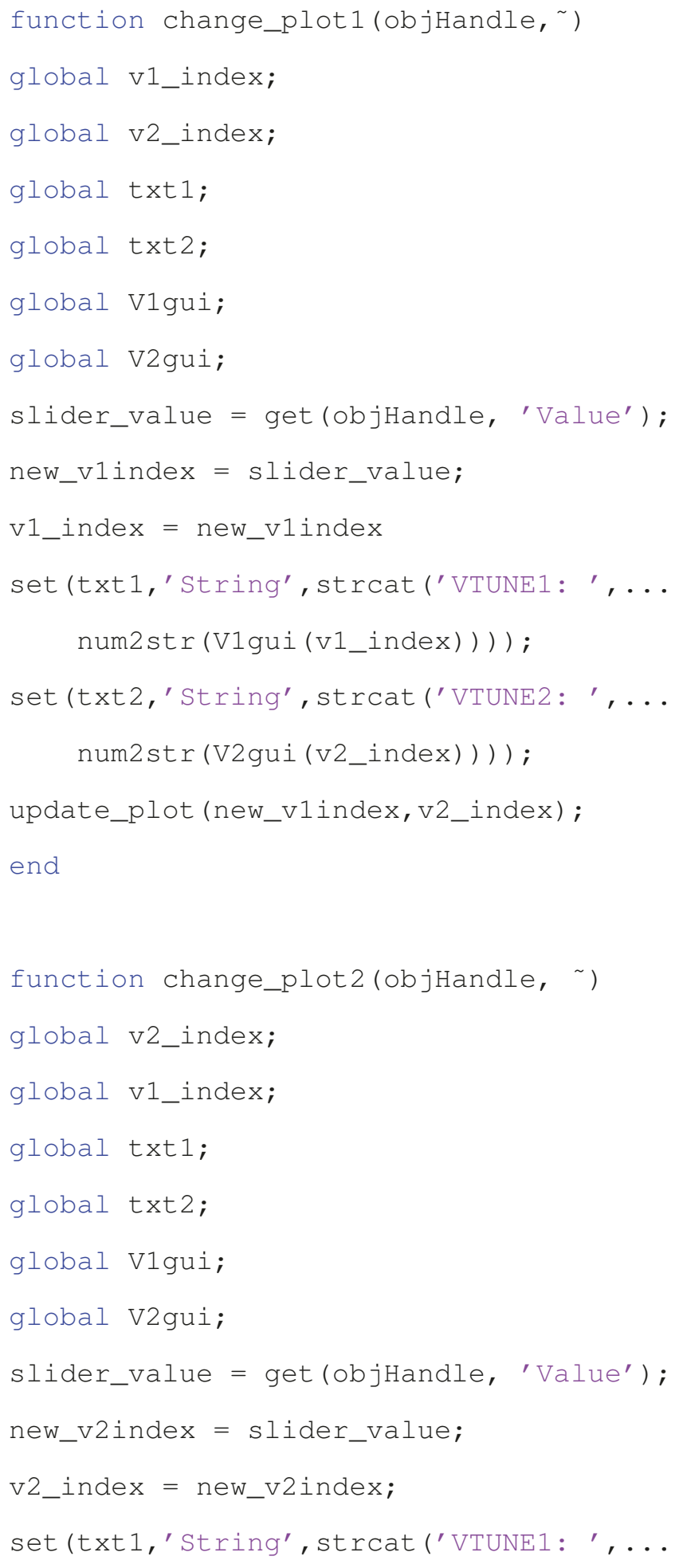




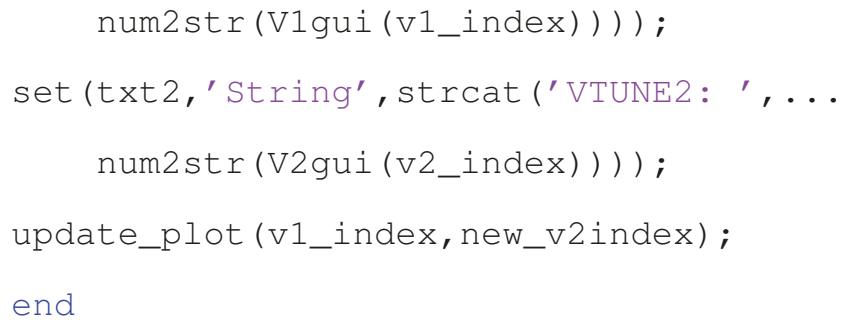




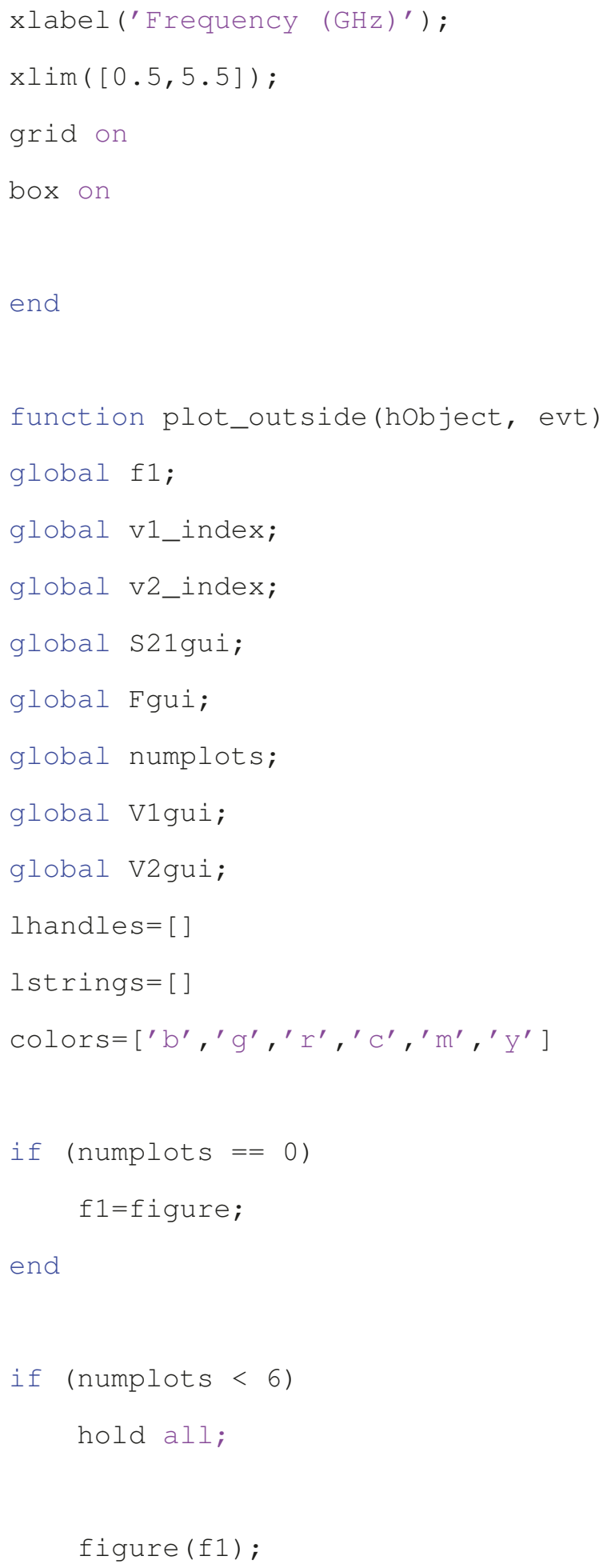




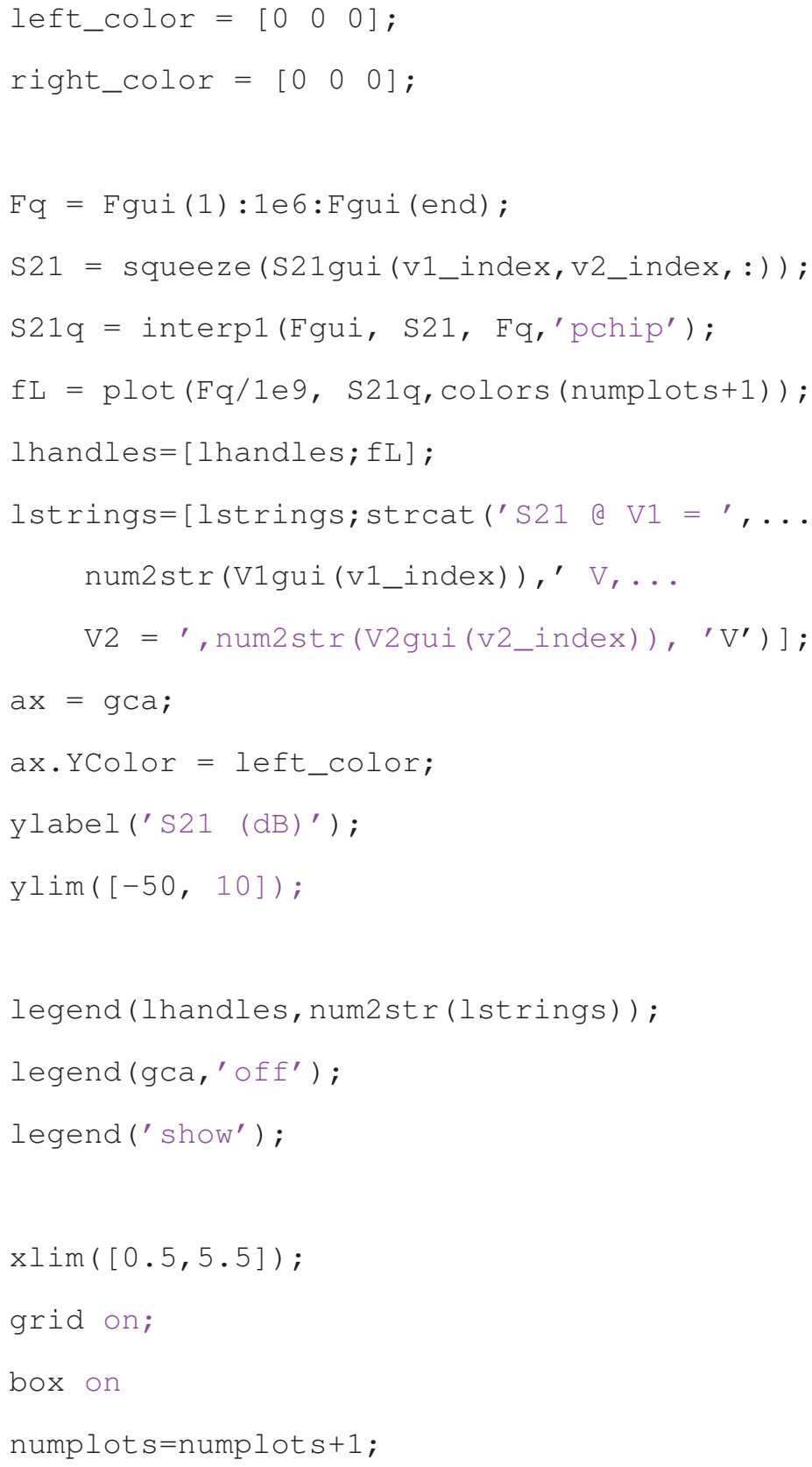

